\title{
RYERSON UNIVERISTY
}

\section{ON THE DEVELOPMENT OF A STRENGTH PREDICTION METHODOLOGY FOR FIBRE METAL LAMINATES IN PIN BEARING}

by

PETER PANAGIOTIS KRIMBALIS, B.Eng., MASc.

\author{
A DISSERTATION \\ PRESENTED TO RYERSON UNIVERSITY \\ IN PARTIAL FULFILLMENT OF \\ THE REQUIREMENTS FOR THE DEGREE OF \\ DOCTOR OF PHILOSOPHY
}

IN THE PROGRAM OF

AEROSPACE ENGINEERING

TORONTO, ONTARIO, CANADA, 2009

(C) PETER P. KRIMBALIS 2009 


\section{RYERSON UNIVERSITY SCHOOL OF GRADUATE STUDIES}

I hereby declare that I am the sole author of this thesis or dissertation.

I authorize Ryerson University to lend this thesis or dissertation to other institutions or individuals for the purpose of scholarly research.

Author, PETER P. KRIMBALIS,

DEPARTMENT OF AEROSPACE ENGINEERING

I further authorize Ryerson University to reproduce this thesis or dissertation by photocopying or by other means, in total or in part, at the request of other institutions or individuals for the purpose of scholarly research.

Author, PETER P. KRIMBALIS,

DEPARTMENT OF AEROSPACE ENGINEERING 


\section{Abstract}

TITLE: ON THE DEVELOPMENT OF A STRENGTH PREDICTION

METHODOLOGY FOR FIBRE METAL LAMINATES IN PIN BEARING

DEGREE: DOCTOR OF PHILOSOPHY, 2009

AUTHOR: PETER PANAGIOTIS KRIMBALIS

INSTITUTION: DEPARTMENT OF AEROSPACE ENGINEERING, RYERSON

\section{UNIVERSITY}

The development of Fibre Metal Laminates (FMLs) for application into aerospace structures represents a paradigm shift in airframe and material technology. By consolidating both monolithic metallic alloys and fibre reinforced composite layers, a new material structure is born exhibiting desired qualities emerging from its heterogeneous constituency. When mechanically fastened via pins, bolts and rivets, these laminated materials develop damage and ultimately fail via mechanisms that were not entirely understood and different than either their metallic or composite constituents. The development of a predictive methodology capable of characterizing how FMLs fastened with pins behave and fail would drastically reduce the amount of experimentation necessary for material qualification and be an invaluable design tool.

The body of this thesis discusses the extension of the characteristic dimension approach to FMLs and the subsequent development of a new failure mechanism as part of a progressive damage finite element (FE) modeling methodology with yielding, 
delamination and buckling representing the central tenets of the new mechanism. This yielding through delamination buckling (YDB) mechanism and progressive FE model were investigated through multiple experimental studies. The experimental investigations required the development of a protocol with emphasis on measuring deformation on a local scheme in addition to a global one. With the extended protocol employed, complete characterization of the material response was possible and a new definition for yield in a pin bearing configuration was developed and subsequently extended to a tensile testing configuration. The performance of this yield definition was compared directly to existing definitions and was shown to be effective in both quasi-isotropic and orthotropic materials.

The results of the experiments and FE simulations demonstrated that yielding (according to the new definition), buckling and delamination resulting in joint collapse and failure have all occurred within the stipulated predictions of the YDB mechanism. 


\section{Acknowledgements}

I would like to express my sincerest gratitude to my supervisor, Professor Cheung Poon who is, without a shadow of a doubt, the epitome of what a thesis supervisor should be. I thank you for your wisdom, guidance, encouragement and character as a role model. These are the precise qualities that greatly facilitated the completion of this thesis and will invariably aid me in the commencement of my career.

I am truly thankful to my co-supervisors, Professor Zouheir Fawaz and Professor Kamran Behdinan. It is only through their tireless efforts to develop the infrastructure of the FRAMES laboratory and the Department of Aerospace Engineering in general, that it was possible to even begin this work. It has been an absolute pleasure working with you. I am deeply thankful to the Natural Science and Engineering Research Council (NSERC) for providing me with the funding for my $\mathrm{PhD}$ through their Canada Graduate Scholarship (CGS-D2) and generously funding my supervisors through their Discovery Grant. I also gratefully acknowledge the Ontario Graduate Scholarship (OGS), the Canadian Foundation for Innovation (CFI), the Ontario Innovation Trust (OIT) and Bombardier Aerospace whose generous funding and donation of materials continuously enables the fulfilment of the research goals of the FRAMES laboratory.

I am forever indebted to my fiancée, Ms. Susan Chen, who experienced by proxy, more than anyone else, what exactly it took to complete this $\mathrm{PhD}$. Susy, your sage-like wisdom in reminding me of who I am and your presence of mind to opportunely distract me for the preservation of my sanity are the single greatest reasons why I embarked upon and 
ultimately completed this final scholastic endeavour. I promise Bebe, this was the last one.

I am very thankful to my brother, Mr. Jim Krimbalis, P.Eng., who has been a pillar of strength and support for my entire life and was the reason why I considered engineering in the first place. Thank you Jim, everyone could use an older brother like you. I am eternally grateful for the love and support of my parents Mr. Spyridon and Mrs. Anthoula Krimbalis and my Godmother, Mrs. Fani Tzortzis, who since birth have fanned

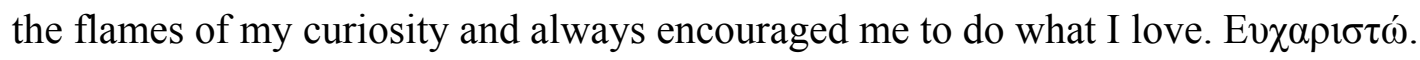
I would like to extend my sincerest thank you to Dr. Hamid Ghaemi and Mr. Primoz Cresnik. Gentlemen, you have influenced me more than you can imagine. Our 8 AM coffee breaks were equal parts a lesson in research and in life; for that I thank you. I am grateful for the efforts and assistance put forth by Mr. Gerald Bootes, Mr. Jerry Karpynczyk and Mr. Peter Bradley. Their technical support proved invaluable on occasions too numerous to mention. Not to be forgotten, I thank Mr. Alan Machin for his most generous assistance and training in the preparation of the SEM specimens and for allowing me to intrude into his laboratory. Many thanks to the administrative staff in both the Department of Aerospace Engineering and the School of Graduate Studies, specifically Mrs. Kim Gallo and Mrs. Leah Rogan for their ongoing support from my undergraduate studies all the way through to the present. Also, I wanted to thank Dr. Jeffrey Yokota for the numerous honest and candid conversations we had. My only regret is not getting to know you sooner. Finally, I would like to express my most heartfelt gratitude to Ryerson University which has, for the better part of a decade, literally been my home away from home. 


\section{Dedication}

Dedicated to my loving Susy and our puppy, the distinguished Molly Cosine Chen

Krimbalis a.k.a "Momotron", for relieving me of one flavour of stress only to replace it with another.

In luce vive, vive in amore. 


\section{Table of Contents}

Abstract

CHAPTER ONE: INTRODUCTION AND LITERATURE REVIEW …......................

1.1 Historic Development of FML Technology ......................................................2

1.2 Constituent Materials and Mechanical Behaviour of FMLs.....................................5

1.2.1 Constituent Materials and Manufacturing Process ............................................5

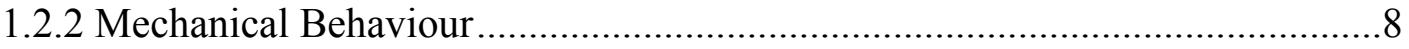

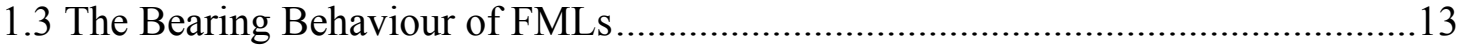

1.4 Failure Prediction and Modeling of FMLs .............................................................

1.4.1 The Characteristic Dimension Method........................................................... 19

1.4.2 FE Modeling of FMLs.............................................................................21

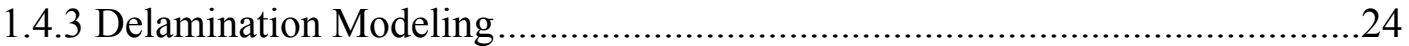

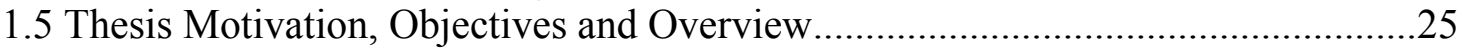

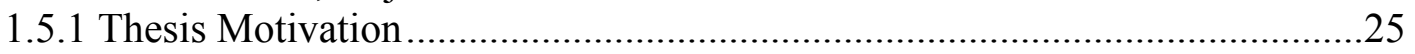

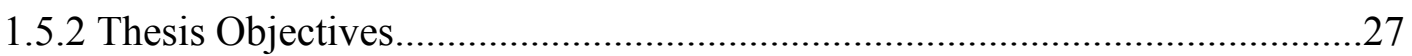

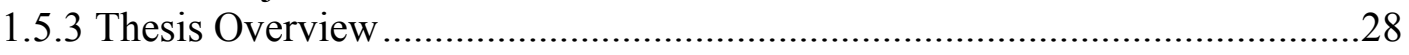

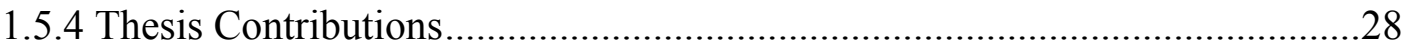

CHAPTER TWO: MODIFIED CHARACTERISTIC DIMENSION AND FAILURE

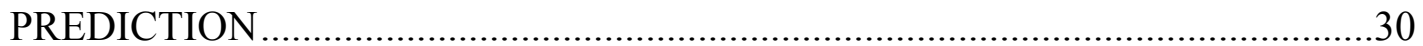

2.1 Definition of a Modified CCD and Proposed Yielding Through Delamination

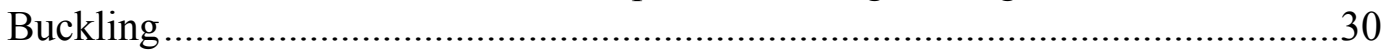

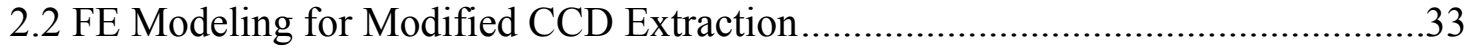

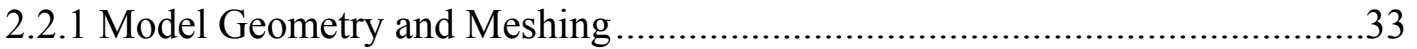

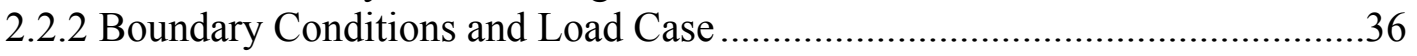

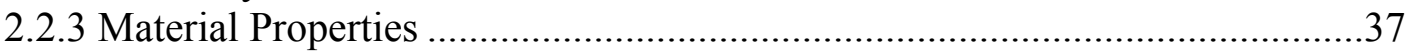

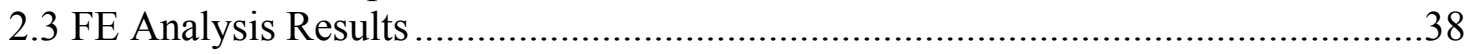

2.3.1 Plot Generation via User Defined Nodal Paths ..........................................40

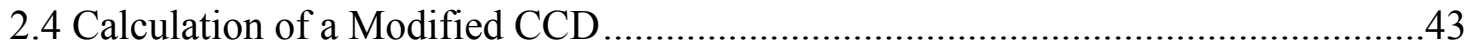

2.5 Orthotropic Plate Buckling Analysis of the Laterally Unsupported Glass

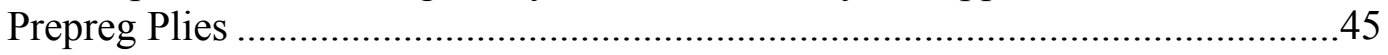

2.5.1 Results of the Orthotropic Plate Buckling Analysis....................................49

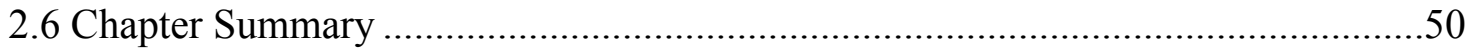




\section{CHAPTER THREE: BEARING STRENGTH AND TENSILE TESTS ON QUASI-}

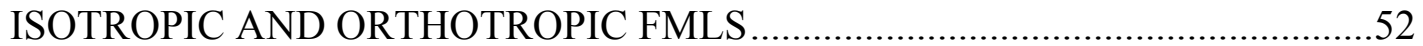

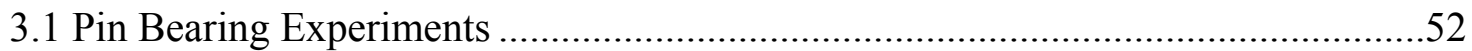

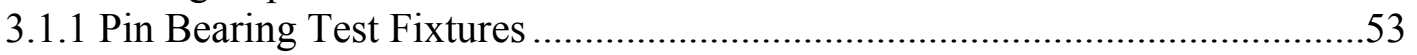

3.1.2 Fabrication of Tested Samples ...................................................................5

3.1.3 Pin Bearing Experimental Protocol .............................................................60

3.2 Results and Discussion of the Pin Bearing Experiments ........................................64

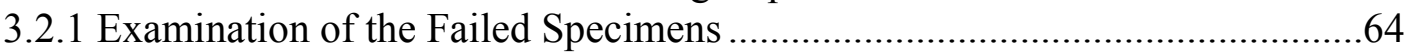

3.2.2 Results of the Globally Applied Measurements ..............................................66

3.2.3 Calculation of Standard Bearing Yield Strength ...............................................68

3.2.4 Results of the Locally Applied Measurements.................................................69

3.2.5 Characterization of the Locally Measured Bearing Stress-Strain Curves ........72

3.2.6 Calculation of a Locally Measured Bearing Yield Strength ............................79

3.2.7 Detection of Buckling in the Aluminum Layers ............................................83

3.2.8 Analysis of Buckling in the Aluminum Layers ..............................................84

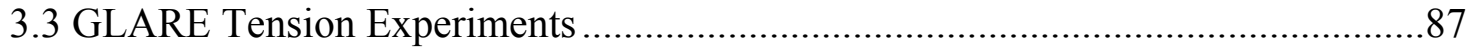

3.3.1 GLARE Tension Test Specimen Fabrication and Preparation.........................87

3.3.2 Experimental Protocol for the GLARE Tension Tests....................................90

3.4 Results and Discussion of the GLARE Tension Tests ..........................................90

3.4.1 Calculation of Standardized Parameters..........................................................94

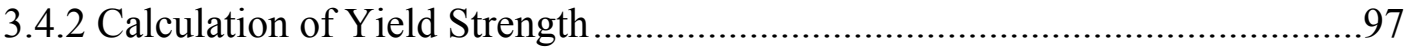

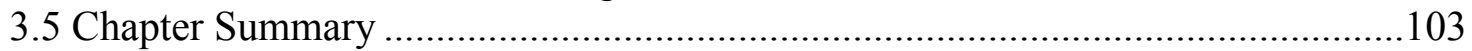

\section{CHAPTER FOUR: PROGRESSIVE DAMAGE FE MODELING OF GLARE FMLS}

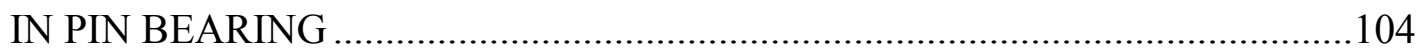

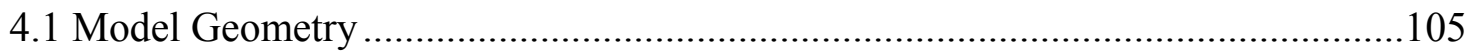

4.2 Material Considerations and Implementation....................................................107

4.2.1 2024-T3 Aluminum with Isotropic Strain Hardening ....................................107

4.2.2 FM-94 Epoxy Adhesive: Elastic Response, Damage Initiation, Evolution

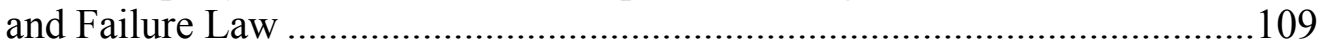

4.2.2.1 Implementation of a Traction Separation Law for a Cohesive

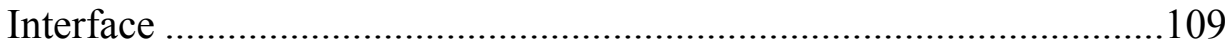

4.2.2.2 Implementation of Damage Initiation for the Cohesive Interface ........113

4.2.2.3 Implementation of Damage Evolution for a Cohesive Interface .........115

4.2.3 $\mathrm{S}_{2}$-Glass Fibre Composite Prepreg with Damage Initiation and Evolution ..119

4.2.3.1 Undamaged Elastic Response .........................................................119

4.2.3.2 Implementation of Damage Initiation for Elastic-Brittle Materials Exhibiting Anisotropic Behaviour ......................................................120

4.2.3.3 Implementation of Damage Evolution for Fibre-Reinforced Composites Exhibiting Elastic-Brittle Behaviour................................122

4.3 Implementation of Thermally Induced Residual Stresses ......................................123

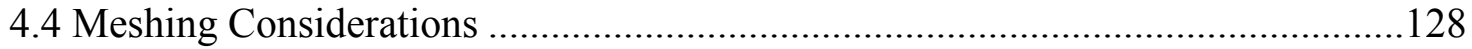

4.4.1 Meshing Considerations for the Aluminum Layers .....................................131

4.4.2 Meshing Considerations for the Cohesive Interface ......................................132

4.4.3 Meshing Considerations for the Glass Prepreg Layers .................................133

4.5 Analysis Steps, Boundary Conditions and Load Case..........................................135

4.6 Requested Field/History Output Variables .................................................................141 
4.7 Chapter Summary

CHAPTER FIVE: RESULTS AND DISCUSSION OF THE PROGRESSIVE

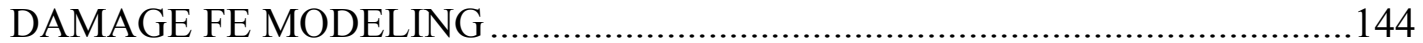

5.1 Results and Discussion of the Thermally Induced Residual Stresses.....................145

5.1.1 Results of the Analytical Calculations of Thermally Induced Residual Stress

5.1.2 Results of the FE Simulation of Thermally Induced Residual Stress

5.1.3 Comparison and Discussion of FE Results to Analytical Calculations and the Literature

5.2 Results and Discussion of the Applied Loading in the Progressive Damage FE Modeling

5.2.1 Results of the Applied Load Step.

5.2.2 Results and Discussion for the Aluminum Layers 164

5.2.2.1 Results from Additional Field Output Variables: ACYIELD, PE and PEEQ.

5.2.3 Results and Discussion for the Glass Layers

5.2.4 Results and Discussion of the Delamination Modeling 186

5.3 SEM Imagery of Failed Specimens and Comparison to Progressive Damage FE Model

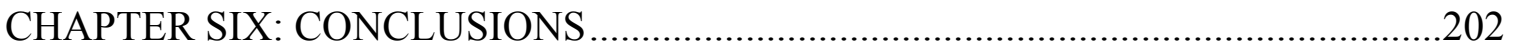

CHAPTER SEVEN: FUTURE CONSIDERATIONS …………………………........204

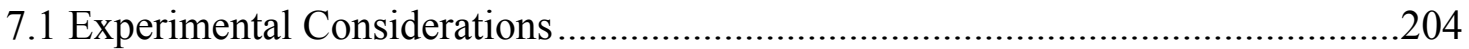

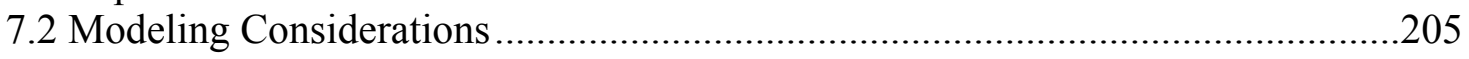

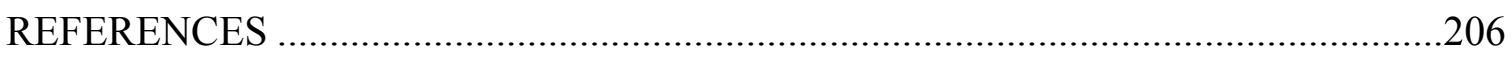

APPENDIX A: ORTHOTROPIC PLATE BUCKLING ANALYSIS .............................221

APPENDIX B: ISOTROPIC PLATE BUCKLING ANALYSIS ………………….......226

APPENDIX C: EXTRACTION OF THE ETA PARAMETER IN THE B-K

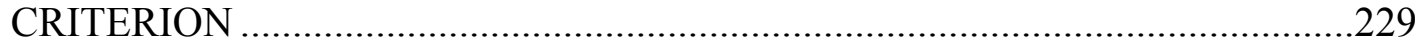

APPENDIX D: NOMINAL VALUE THRESHOLD EXTRACTION ............................230

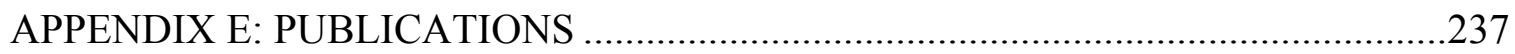




\section{List of Tables}

Table 1.1: A summary of the commercially available GLARE variants and their constituent materials.

Table 1.2: A summary of some of the static properties of several GLARE variants (adapted from $[21,28,29]$ )

Table 2.1: Experimental bearing strength values from Buemler [64] in columns 1, 3 and 4 and van Rooijen et. al. [58] in column 2.................................................. 34

Table 2.2: A summary of GLARE variants analyzed and their respective dimensions/lay-ups.

Table 2.3: A summary of the calculated failure loads and loads applied in the symmetry models

Table 2.4: Material properties for aluminum 2024-T3 and $\mathrm{S}_{2}$-glass fibre. 37

Table 2.5: Calculated modified CCD for one stress component and two combined stresses for all GLARE variants analyzed.

Table 2.6: Dimensions of the delaminated/laterally unsupported regions of the glass prepreg layers used in the orthotropic plate buckling analysis.

Table 2.7: Critical buckling constants, loads and normal stresses for the clampedclamped-clamped-simply supported loading configuration.

Table 2.8: Critical buckling constants, loads and normal stresses for the fully clamped loading configuration.

Table 3.1: A summary of GLARE variants tested and their respective dimension/layups.

Table 3.2: Summary of the mean bearing loads, standard deviation and associated bearing yield strength, corresponding to a $4 \%$ permanent hole deformation.

Table 3.3: Summary of the calculated intersection angle for GLARE4.

Table 3.4: Summary of the load, strain and stress as calculated by the bisection protocol for all GLARE variants tested.

Table 3.5: Summary of the maximum bearing load and associated bearing stress for a GLARE4 variant. 
Table 3.6: Summary of the test matrix employed. Note: $b t b$ indicates back to back gages were applied.

Table 3.7: Calculated ultimate tensile stress and its associated statistics for all GLARE variants tested.

Table 3.8: Calculated tensile chord modulus and associated statistics as measured by the longitudinal strain gage and the extensometer................................................. 95

Table 3.9: Calculated Poisson's ratio for all GLARE variants tested............................ 95

Table 3.10: Calculated percent bending as measured by the back to back gages for all

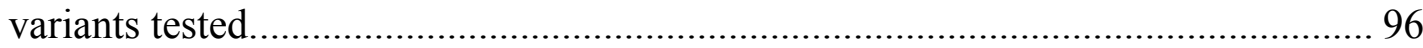

Table 3.11: Summary of the calculated tensile yield strength as measured by the longitudinal strain gage and the extensometer according to the bisection protocol. 98

Table 3.12: Calculated intersection angle as defined by the bisection protocol. 98

Table 3.13: Summary of the calculated tensile yield strength as measured by the longitudinal strain gage and the extensometer according to the bilinear idealization. 101

Table 3.14: Summary of the calculated tensile yield strength as measured by the longitudinal strain gage and the extensometer according to the $0.2 \%$ offset.

Table 3.15: Relative error in calculated tensile yield strength between the bisection protocol, the idealized bilinear approach and the $0.2 \%$ offset technique.

Table 4.1: A summary of the GLARE variants analyzed and their respective dimensions/lay-ups.

Table 4.2: Elastic and plastic properties of 2024-T3 aluminum. 108

Table 4.3: Calculated elastic parameters for the traction-separation response of FM94 epoxy adhesive.

Table 4.4: Nominal strain parameters required for the quadratic nominal strain criterion within the damage initiation model.

Table 4.5: Mode-mixity and strain energy release rates as for a glass/epoxy composite [105].

Table 4.6: Summary of the elastic properties of the $S_{2}$-glass prepreg layers. 120

Table 4.7: Summary of the failure strengths employed for the glass prepreg layers 121 
Table 4.8: Critical energy release rates for the fibre and matrix in both tension and

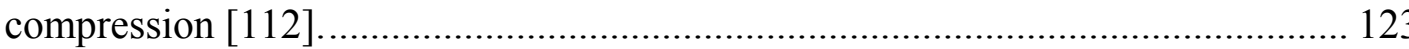

Table 4.9: Summary of the properties required to calculate the residual stresses ......... 128

Table 4.10: Summary of the analysis steps employed, their time periods, consideration of non-linear effects and incrementation.

Table 4.11: Summary of the applied boundary conditions and their state changes throughout the analysis steps.

Table 4.12: Summary of the model specific enforced contact displacements applied after the residual stress step.

Table 5.1: Calculated thermally induced residual stresses in both the aluminum and glass layers using the isotropic assumption.

Table 5.2: Calculated thermally induced residual stresses in both the aluminum and glass layers assuming orthotropic glass layers

Table 5.3: Comparison of the analytical, FE and literature residual stress results for the aluminum layers.

Table 5.4: Comparison of the analytical, FE and literature residual stress results for the glass prepreg layers

Table 5.5: Summary of the loads applied to the progressive damage FE models as taken from the experimental study and bisection protocol yield definition.

Table 5.6: Summary of the extracted distances to nominal ACYIELD values and their relative error from the predicted modified CCD for a GLARE4-3/2-0.3 variant... 175

Table 5.7: Summary of the average distances to the nominal ACYIELD value and the relative error from the variant specific modified $\mathrm{CCD}$ value for all variants.

Table 5.8: Summary of the extracted distances to nominal PE and PEEQ values and their relative error from the predicted modified CCD for a GLARE4-3/2-0.3 variant.

Table 5.9: Summary of the average distances to the nominal PE and PEEQ value and the relative error from the variant specific modified CCD value for all variants... 182

Table 5.10: Summary of the average delamination damage distance compared to the variant specific modified CCD calculation....

Table 5.11: Summary of the measured delaminated distances and some associated statistics 
Table 5.12: A comparison of the predicted delaminated distances as per the FE model and the measured delaminated distances from the SEM imagery. ......................... 200 


\section{List of Figures and Illustrations}

Figure 1.1: A typical FML lay-up depicting both the metallic and fibrous layers (adapted from [20]).

Figure 1.2: Schematic representation of the conventional characteristic dimension method for the compressive characteristic dimension.

Figure 1.3: Schematic representation of the conventional characteristic dimension method for the tensile characteristic dimension........................................... 20

Figure 1.4: Schematic description of the characteristic curve with angle $\theta$ shown......... 22

Figure 1.5: Location of GLARE panels implemented on the Airbus A380 aircraft

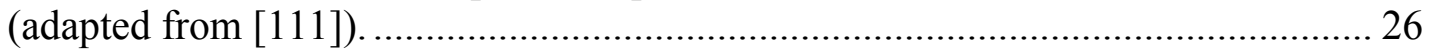

Figure 2.1: Schematic representation of the modified CCD....................................... 31

Figure 2.2: Model geometry with a typical mesh and hardened steel lateral restraints for pin bearing analysis.

Figure 2.3: Typical Von Mises stress distribution for GLARE 2-2/1-0.4 showing region of highest compressive stress........................................................... 38

Figure 2.4: Typical Von Mises stress distribution for GLARE3-5/4-0.4 showing region of highest compressive stress.

Figure 2.5: Profile of GLARE3-5/4-0.4 showing the buckling of aluminum layers with a scale factor of 25 for clarity.

Figure 2.6: User defined nodal path parallel to the 1 (longitudinal) axis in the aluminum layer of a typical pin bearing model.

Figure 2.7: Comparison of the Von Mises stress distribution in the aluminum layers for all GLARE variants analyzed.

Figure 2.8: Comparison of the Von Mises stress distribution in the glass layers in both the longitudinal and transverse directions for all GLARE variants analyzed.

Figure 2.9: Schematic of the delaminated/laterally unsupported area used in the orthotropic plate buckling analysis based on the results of the FE analysis.

Figure 3.1: Tension based loading fixture with machined slot for the facilitation of strain gage application on the quasi-isotropic specimens. 
Figure 3.2: Hardened steel side plate with expanded slot as part of the tension based loading fixture for the orthotropic specimens...................................................... 54

Figure 3.3: Typical pin bearing GLARE specimen with its major dimensions shown. ... 56

Figure 3.4: Typical quasi-isotropic bearing test specimen illustrating the location and orientation of the bonded strain gage.................................................................. 58

Figure 3.5: Typical orthotropic bearing test specimen with the location and orientation of the bonded strain gage shown.

Figure 3.6: Typical quasi-isotropic bearing test specimen with the loading pin inserted as part of the tension based loading fixture.

Figure 3.7: MTS 322 load frame with quasi-isotropic GLARE specimen mounted in the test fixture and extensometer in place for the measurement of relative pin displacement.

Figure 3.8: Typical quasi-isotropic specimen failing in a bearing mode. Note the elongation of the fastener hole and the significant amount of delamination/yielding, some of which is out of plane.

Figure 3.9: Typical failed specimen of GLARE4 exhibiting an elongated hole with extensive delamination, yielding and buckling clearly visible.

Figure 3.10: Typical failed specimen of GLARE4 from a reverse perspective exhibiting an elongated hole with extensive delamination, yielding and buckling clearly visible.

Figure 3.11: Bearing load versus extensometer deformation curves for GLARE3-5/40.3 .

Figure 3.12: Bearing load versus extensometer deformation curves for GLARE3-4/30.3

Figure 3.13: Calculated bearing stress versus measured strain curves for GLARE35/4-0.3.

Figure 3.14: Calculated bearing stress versus measured strain curves for GLARE34/3-0.3.

Figure 3.15: Calculated bearing stress versus measured longitudinal strain curves for GLARE4-3/2-0.3.

Figure 3.16: Calculated bearing stress versus measured longitudinal strain curves for GLARE4-3/2-0.3. 
Figure 3.17: Bearing stress vs. measured strain curve for a GLARE3-4/3-0.3 specimen showing the linear approximations to the low and high strain regimes.... 74

Figure 3.18: Non-linear portion of the bearing stress vs. measured strain curve for a GLARE3-5/4-0.3 specimen showing the logarithmic approximation. 76

Figure 3.19: Bearing stress vs. measured strain curve for a GLARE3-4/3-0.3 specimen showing the piecewise characterization. 78

Figure 3.20: Bearing stress vs. measured strain curve for a GLARE4-3/2-0.3 specimen showing the piecewise characterization. 78

Figure 3.21: The bisection protocol as applied to a quasi-isotropic GLARE3-5/4-0.3 specimen. The magnified view shows the near-orthogonality of the intersection.... 80

Figure 3.22: Bearing stress vs. measured strain curve for a GLARE4-3/2-0.3 specimen showing both of the linear approximations, the logarithmic approximation and the bisection line as required for the definition of bearing yield strength.

Figure 3.23: Bearing stress vs. measured strain curves for both the longitudinally and transversely oriented gages. 84

Figure 3.24: Dimensions of a typical GLARE FML tension testing specimen. 88

Figure 3.25: Typical GLARE tension test specimen with biaxial strain gage applied..... 89

Figure 3.26: MTS 322 load frame with GLARE specimen mounted and extensometer/strain gage in place for the measurement of specimen deformation. . 91

Figure 3.27: Tensile stress versus strain as measured by the bonded strain gage for all tested specimens of a GLARE3-4/3-0.3 variant.

Figure 3.28: Tensile stress versus strain as measured by the bonded strain gage for all tested specimens of a GLARE3-5/4-0.3 variant.

Figure 3.29: Tensile stress versus strain as measured by the bonded strain gage for all tested specimens of a GLARE4-3/2-0.3 variant.

Figure 3.30: Direct comparison of material behaviour as measure by the extensometer and longitudinal gage for a GLARE3-4/3-0.3 variant.

Figure 3.31: Piecewise characterization of the tensile stress versus strain profile of a typical GLARE variant.

Figure 3.32: Bisection protocol yield definition as applied to a typical GLARE variant

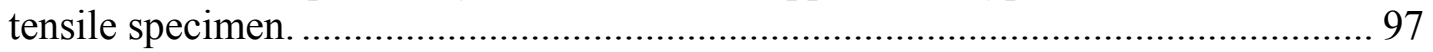

Figure 3.33: Bilinear idealization as performed on a GLARE3-4/3-0.3 variant. 99 
Figure 3.34: The $0.2 \%$ offset technique as performed on a GLARE4-3/2-0.3 variant... 100

Figure 4.1: Global geometry of a typical model with loading pin and hardened steel

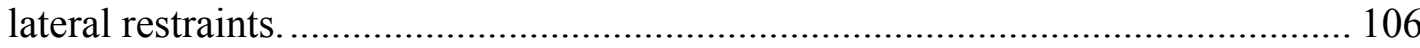

Figure 4.2: Modified hardened steel lateral restraint for application to the GLARE43/2-0.3 specimen highlighting the modified machined slot.................................. 106

Figure 4.3: Global assembly mesh for a typical variant analyzed............................. 129

Figure 4.4: Typical local mesh for the aluminum layers only................................. 130

Figure 4.5: Typical local mesh for the cohesive interface layers. ............................. 130

Figure 4.6: Typical local mesh for the glass prepreg layers. ................................... 131

Figure 4.7: Applied boundary conditions on a typical variant analyzed. ..................... 137

Figure 5.1: Distribution within the aluminum layers of the Von Mises field output variable for the developed residual stresses in a GLARE3-5/4-0.3 variant........... 148

Figure 5.2: Distribution within the aluminum layers of the Von Mises field output variable for the developed residual stresses in a GLARE3-4/3-0.3 variant........... 148

Figure 5.3: Distribution of the Von Mises field output variable within the aluminum layer adjacent to a transverse glass ply bound on one side for the developed residual stresses in a GLARE4-3/2-0.3 variant.

Figure 5.4: Distribution of the Von Mises field output variable within the aluminum layer adjacent to a transverse glass ply bound on both sides for the developed residual stresses in a GLARE4-3/2-0.3 variant.

Figure 5.5: Distribution of the Von Mises field output variable for the developed residual stresses in a longitudinally oriented glass prepreg layer in a GLARE35/4-0.3 variant.

Figure 5.6: Distribution of the Von Mises field output variable for the developed residual stresses in a transversely oriented glass prepreg layer in a GLARE3-5/40.3 variant.

Figure 5.7: Through the thickness distribution of the Von Mises field output variable for the developed residual stresses in all the glass prepreg layers in a GLARE35/4-0.3 variant.

Figure 5.8: Through the thickness distribution of the Von Mises field output variable for the residual stresses in all the glass prepreg layers in a GLARE3-4/3-0.3 variant. Note the asymmetric distribution revealing the transverse bias. 
Figure 5.9: Distribution of the Von Mises field output variable for the developed residual stresses in a longitudinally oriented glass prepreg layer in a GLARE43/2-0.3 variant.

Figure 5.10: Distribution of the Von Mises field output variable for the developed residual stresses in a transversely oriented glass prepreg layer adjacent to the outermost layer of aluminum in a GLARE4-3/2-0.3 variant.

Figure 5.11: Distribution of the Von Mises field output variable for the developed residual stresses in a transversely oriented glass prepreg layer adjacent to the innermost layer of aluminum in a GLARE4-3/2-0.3 variant.

Figure 5.12: Through the thickness distribution of the Von Mises field output variable for the residual stresses in all the glass prepreg layers in a GLARE4-3/2-0.3 variant.

Figure 5.13: Distribution of Von Mises stress in the cohesive layers of a GLARE34/3-0.3 variant.

Figure 5.14: Through the thickness distribution of induced Von Mises stress in the cohesive interfaces of a GLARE3-5/4-0.3 variant.

Figure 5.15: Distribution of the QUADECRT field output variable indicating the onset of delamination due to residual stress induction in a cohesive layer within a GLARE4-3/2-0.3 variant. 160

Figure 5.16: Through the thickness distribution of the QUADECRT field output variable as a result of induced residual stresses in a GLARE4-3/2-0.3 variant. Note that damage to the interface, though slight, has already occurred.

Figure 5.17: Calculated contact pressure between the pin and the laminate at the end of the applied load step for a GLARE3-5/4-0.3 laminate.

Figure 5.18: Calculated contact pressure between the pin and the laminate at the end of the applied load step for a GLARE3-4/3-0.3 laminate.

Figure 5.19: Calculated contact pressure between the pin and the laminate at the end of the applied load step for a GLARE4-3/2-0.3 laminate.

Figure 5.20: Distribution of the Von Mises stress field output variable in the aluminum layers resulting from the applied pin bearing load in a GLARE3-5/40.3 laminate.

Figure 5.21: Through the thickness distribution of the Von Mises stress field output variable in the aluminum layers resulting from the applied pin bearing load in a GLARE3-5/4-0.3 laminate. 
Figure 5.22: Distribution of the logarithmic strain in the longitudinal direction field output variable in the aluminum layers resulting from the applied pin bearing load in a GLARE3-5/4-0.3 laminate.

Figure 5.23: Distribution of the Von Mises stress field output variable in the aluminum layers resulting from the applied pin bearing load in a GLARE3-4/30.3 laminate. 166

Figure 5.24: Distribution of the logarithmic strain in the longitudinal direction field output variable in the aluminum layers resulting from the applied pin bearing load in a GLARE3-4/3-0.3 laminate.

Figure 5.25: Distribution of the Von Mises stress field output variable in the aluminum layers resulting from the applied pin bearing load in a GLARE4-3/20.3 laminate.

Figure 5.26: Distribution of the logarithmic strain in the longitudinal direction field output variable in the aluminum layers resulting from the applied pin bearing load in a GLARE4-3/2-0.3 laminate.

Figure 5.27: Distribution of the ACYIELD field output variable in the aluminum layers in a GLARE3-5/4-0.3 variant. Note the close similarity of the pattern of damage to the experimental specimens.

Figure 5.28: Through the thickness distribution of the ACYIELD field output variable in the aluminum layers of a GLARE3-5/4-0.3 variant.

Figure 5.29: Distribution of the ACYIELD field output variable in the aluminum layers in a GLARE3-4/3-0.3 variant.

Figure 5.30: Distribution of the ACYIELD field output variable in the aluminum layers in a GLARE4-3/2-0.3 variant.

Figure 5.31: User defined nodal path within a typical aluminum layer used for the extraction of field output variable requests.

Figure 5.32: Plot of averaged ACYIELD versus distance from fastener hole for a GLARE3-5/4-0.3 variant. Note the distance to which the nominal value is reached.

Figure 5.33: Plot of averaged ACYIELD versus distance from fastener hole for a GLARE3-4/3-0.3 variant. Note the distance to which the nominal value is reached.

Figure 5.34: Plot of averaged ACYIELD versus distance from fastener hole for a GLARE4-3/2-0.3 variant. Note the distance to which the nominal value is reached. 
Figure 5.35: Distribution of the PEEQ field output variable within the aluminum layers of a GLARE3-5/4-0.3 variant. 178

Figure 5.36: Distribution of the PE field output variable within the aluminum layers of a GLARE4-3/2-0.3 variant. 178

Figure 5.37: Distribution of the PEEQ field output variable within the aluminum layers of a GLARE4-3/2-0.3 variant. . 179

Figure 5.38: Plot of averaged PE and PEEQ versus distance from fastener hole for a GLARE3-5/4-0.3 variant.

Figure 5.39: Plot of averaged PE and PEEQ versus distance from fastener hole for a GLARE3-4/3-0.3 variant.

Figure 5.40: Plot of averaged PE and PEEQ versus distance from fastener hole for a GLARE4-3/2-0.3 variant.

Figure 5.41: Distribution of the Von Mises stress field output variable for a longitudinal glass prepreg layer in a GLARE3-4/3-0.3 variant.

Figure 5.42: Distribution of the Von Mises stress field output variable for a transverse glass prepreg layer in a GLARE3-4/3-0.3 variant.

Figure 5.43: Distribution of the HSNFCCRT field output variable in a longitudinal glass prepreg layer of a GLARE4-3/2-0.3 variant.

Figure 5.44: Distribution of the HSNMCCRT field output variable in a longitudinal glass prepreg layer of a GLARE3-4/3-0.3 variant.

Figure 5.45: Distribution of the HSNMCCRT field output variable in a transverse glass prepreg layer of a GLARE3-4/3-0.3 variant.

Figure 5.46: Typical distribution of the Von Mises field output variable in a cohesive interface layer of a GLARE4-3/2-0.3 variant.

Figure 5.47: Distribution of delamination in the cohesive interface layer of a GLARE3-5/4-0.3 variant.

Figure 5.48: Distribution of delamination in the cohesive interface layer of a GLARE3-4/3-0.3 variant. 188

Figure 5.49: Distribution of delamination in the cohesive interface layer of a GLARE4-3/2-0.3 variant.

Figure 5.50: Schematic illustrating the measurement of delaminated distance within a cohesive interface layer. 
Figure 5.51: Plot of delamination damage versus increasing distance from fastener hole for a GLARE3-5/4-0.3 variant.

Figure 5.52: Plot of delamination damage versus increasing distance from fastener hole for a GLARE3-4/3-0.3 variant.

Figure 5.53: Plot of delamination damage versus increasing distance from fastener hole for a GLARE4-3/2-0.3 variant.

Figure 5.54: SEM image of a GLARE3-4/3-0.3 variant with delamination measurement.

Figure 5.55: SEM image of a GLARE4-3/2-0.3 variant with delamination measurement.

Figure 5.56: SEM image of a GLARE3-4/3-0.3 variant with delamination measurement.

Figure 5.57: SEM image of a GLARE3-5/4-0.3 variant with delamination measurement. 195

Figure 5.58: End point of delamination damage within a GLARE4-3/2-0.3 variant. .... 196

Figure 5.59: End point of delamination damage within a GLARE4-3/2-0.3 variant. .... 197

Figure 5.60: End point of delamination damage within a GLARE3-4/3-0.3 variant. .... 197

Figure 5.61: Highly magnified view of a delaminated portion within a typical GLARE variant. Note the combination of pulling and shearing, characteristic of mixed-mode damage. 198 


\section{List of Symbols, Abbreviations and Nomenclature}

\begin{tabular}{|c|c|}
\hline Abbreviations and Acronyms & Definition \\
\hline ARALL & Aramid Reinforced Aluminum Laminate \\
\hline ASTM & American Society for Testing and Materials \\
\hline B-K & Benzeggagh and Kenane criterion \\
\hline BTB & Back To Back \\
\hline CARALL & Carbon Reinforced Aluminum Laminate \\
\hline CCD & Compressive Characteristic Dimension \\
\hline CFRP & Carbon Fibre Reinforced Polymer \\
\hline DCB & Double Cantilever Beam \\
\hline FE & Finite Elements \\
\hline FML & Fibre Metal Laminate \\
\hline GLARE & Glass Reinforced Aluminum Laminate \\
\hline GFRP & Glass Fibre Reinforced Polymer \\
\hline JSF & Joint Strike Fighter \\
\hline LR & Lateral Restraint \\
\hline MVF & Metal Volume Fraction \\
\hline SEM & Scanning Electron Microscope \\
\hline TCD & Tensile Characteristic Dimension \\
\hline $\mathrm{TiGr}$ & Titanium Graphite Reinforced Laminate \\
\hline TUDelft & Technical University of Delft \\
\hline YDB & Yielding through Delamination Buckling \\
\hline Field Output Variables & Definition \\
\hline ACYIELD & Active Yielding \\
\hline ALLCD & Total energy \\
\hline CDISP & Displacement components of contact surfaces \\
\hline CPRESS & Surface pressure of contact surfaces \\
\hline DMICRT & Damage initiation criterion \\
\hline $\mathrm{E}$ & Total true strain components \\
\hline HSNFCCRT & Damage variable for fibre compression \\
\hline HSNFTCRT & Damage variable for fibre tension \\
\hline HSNMCCRT & Damage variable for matrix compression \\
\hline HSNMTCRT & Damage variable for matrix tension \\
\hline $\mathrm{NE}$ & Nominal strain components \\
\hline $\mathrm{PE}$ & Plastic strain components \\
\hline PEEQ & Equivalent plastic strain \\
\hline QUADECRT & Quadratic nominal strain criterion \\
\hline
\end{tabular}

\section{Abbreviations and Acronyms}

ARALL

B-K

BTB

CCD

CFRP

DCB

FML

GLARE

GFRP

JSF

LR

SEM

TCD

TiGr

TUDelft

Field Output Variables

ACYIELD

\section{ALLCD}

CDISP

CPRESS

DMICRT

HSNMCCRT

HSNMTCRT

$\mathrm{NE}$

PE

PEEQ

QUADECRT

\section{Definition}

Aramid Reinforced Aluminum Laminate

American Society for Testing and Materials

Benzeggagh and Kenane criterion

Back To Back

Carbon Reinforced Aluminum Laminate

Compressive Characteristic Dimension

Carbon Fibre Reinforced Polymer

Double Cantilever Beam

Finite Elements

Fibre Metal Laminate

Glass Reinforced Aluminum Laminate

Glass Fibre Reinforced Polymer

Joint Strike Fighter

Lateral Restraint

Metal Volume Fraction

Scanning Electron Microscope

Tensile Characteristic Dimension

Titanium Graphite Reinforced Laminate

Technical University of Delft

Yielding through Delamination Buckling

\section{Definition}

Active Yielding

Displacement components of contact surfaces

Surface pressure of contact surfaces

Damage initiation criterion

Total true strain components

amage variable for fibre compression

Damage variable for fibre tension

Damage variable for matrix compression

Damage variable for matrix tension

ponents

Equivalent plastic strain 
$\mathrm{S}$

SDEG

$\mathrm{U}_{\mathrm{i}}$

$\mathrm{UR}_{\mathrm{i}}$

\section{Latin Symbols}

$a$

By

b

$b_{e l}$

$b_{\text {nonlin }}$

$b_{p l}$

D

$D_{i j}$

$d_{f}^{t}, d_{f}^{c}, d_{m}^{t}, d_{m}^{c}$

E

$E / D$

$E_{A l}$

$E_{b}$

$E_{G l}$

$E_{g l, 0}$

$E_{g l, 90}$

$E_{i j}$

$E_{\text {lam }}$

$E_{t}$

G

$G_{c}$

$G_{i}$

$G_{i c}$

$G_{i j}$

$G_{\text {shear }}$

K

$k$

$K_{n n}$

$K_{s s}$

$K_{t t}$
Total true stress components

Scalar stiffness degradation

Translational displacement components

Rotational displacement components

\section{Definition}

Buckled plate dimension; numerical parameter for $\sigma_{b-\varepsilon}$ curve

Percent out of plane bending

Buckled plate dimension; numerical parameter

for $\sigma_{b-\varepsilon}$ curve

Elastic numerical parameter in the $\sigma_{b-\varepsilon}$ curve

Non-linear numerical parameter in the $\sigma_{b-\varepsilon}$

curve

Plastic numerical parameter in the $\sigma_{b-\varepsilon}$ curve

Specimen fastener hole diameter

Bending stiffness matrix entries

Composite damage coefficients

Elastic modulus

Edge distance-to-diameter ratio

Elastic modulus for aluminum

Bridge excitation voltage

Elastic modulus for glass assuming isotropy

Elastic modulus for glass in the fibre direction

Elastic modulus for glass in the transverse

direction

Local elastic modulus

Effective laminate modulus of elasticity

Tangent modulus

Shear modulus; strain energy release rate

Critical strain energy release rate

Strain energy release rate in a specified mode

Critical strain energy release rate in a specified mode

Shear modulus in specified directions

Strain energy release rate for mode II and

mode III simultaneously

Stress intensity factor; interfacial penalty stiffness

Buckling constant

Elastic interfacial modulus in the normal

direction

Elastic interfacial modulus in the first shear

direction

Elastic interfacial modulus in the second shear 
$L$

$l_{G}$

$m_{e l}$

$m_{p l}$

$N$

$P$

$p$

$P_{c r}$

$P_{G}$

$R$

$R_{c}$

$R_{G}$

$R_{t}$

$S$

$S_{i j}$

$S^{C}$

$S^{T}$

$T$

$\mathrm{t}$

$\mathrm{T}_{\text {cure }}$

$\mathrm{T}_{\mathrm{R}}$

$t_{a l}$

$t_{A l, t o t}$

$\mathrm{t}_{\mathrm{gl}, 0, \text { tot }}$

$\mathrm{t}_{\mathrm{gl}, 90, \text { tot }}$

$t_{\text {lam }}$

$\mathrm{W} / \mathrm{D}$

$W_{G}$

$X^{C}$

$X^{T}$

$Y^{C}$

$Y^{T}$

Greek Symbols

$\alpha$
$\alpha_{A l}$
$\alpha_{g l}$
$\alpha_{g l, 0}$

direction

Longitudinal direction of laminate

Strain gage grid length

Slope of the elastic portion of the $\sigma_{b-\varepsilon}$ curve

Slope of the plastic portion of the $\sigma_{b-\varepsilon}$ curve

Maximum allowable interfacial normal stress

Applied bearing load

Number of aluminum layers

Critical buckling load per unit length

Power density dissipation

Stress ratio

Compressive characteristic dimension

Nominal gage resistance

Tensile characteristic dimension

Stress; maximum allowable interfacial shear stress

Stress in a specified direction

Longitudinal shear strength

Transverse shear strength

Transverse direction of the laminate; maximum allowable interfacial shear stress

Specimen thickness

Curing temperature

Room temperature

Nominal thickness of an aluminum layer

Total thickness of the aluminum layers

Total thickness of the glass layers in the longitudinal direction

Total thickness of the glass layers in the transverse direction

Total laminate thickness

Specimen width-to-diameter ratio

Strain gage grid width

Compressive strength in the fibre direction

Tensile strength in the fibre direction

Compressive strength in the transverse

direction

Tensile strength in the transverse direction

\section{Definition}

Dimensionless buckling parameter

Coefficient of thermal expansion for

aluminum

Coefficient of thermal expansion for glass

Coefficient of thermal expansion for glass in 


$$
\begin{aligned}
& \alpha_{g l, 90} \\
& \beta \\
& \Delta T \\
& \delta_{i} \\
& \delta_{m} \\
& \delta_{\text {shear }} \\
& \delta_{m}^{f} \\
& \delta_{i}^{o} \\
& \delta^{o}{ }_{m} \\
& \varepsilon \\
& \varepsilon_{b} \\
& \varepsilon_{f} \\
& \varepsilon_{\max } \\
& \varepsilon^{e l} \text { nonlin } \\
& \varepsilon_{n}^{f} \\
& \varepsilon_{S}^{f} \\
& \varepsilon_{t}^{f} \\
& \varepsilon_{\text {nonlin }}^{p l} \\
& \eta \\
& v \\
& v_{i j} \\
& \sigma \\
& \sigma_{b} \\
& \sigma_{c r} \\
& \sigma_{r} \\
& \sigma_{b-\varepsilon} \\
& \sigma_{r, A l} \\
& \sigma_{r, g l} \\
& \sigma_{r, g l, 0} \\
& \sigma_{r, g l, 90}
\end{aligned}
$$

the longitudinal direction

Coefficient of thermal expansion for glass in the transverse direction

Dimensionless buckling parameter; modemixity ratio

Temperature differential

Interfacial displacement in the normal and two shear directions

Mixed-mode relative displacement

Vector norm defined by the two in-plane interfacial shear directions

Mixed-mode relative displacement corresponding to complete decohesion Relative displacement at onset of damage in the interfacial normal and two shear directions Relative mixed-mode displacement at onset of damage

Strain; a small but non-zero displacement change

Measured strain from dorsal gage

Measured strain from ventral gage

Maximum strain in the $\sigma_{b-\varepsilon}$ curve

Strain at the elastic threshold of the non-linear regime of the $\sigma_{b-\varepsilon}$ curve

Nominal interfacial strain in the normal direction

Nominal interfacial strain in the first shear direction

Nominal interfacial strain in the second shear direction

Strain at the plastic threshold of the non-linear regime of the $\sigma_{b-\varepsilon}$ curve

Numerical parameter in the B-K criterion

Poisson's ratio

Poisson's ratio in specified directions

Stress

Bearing stress

Critical buckling stress

Thermally induced residual stress

Bearing stress strain curve

Thermally induced residual stress in the aluminum

Thermally induced residual stress in the glass Thermally induced residual stress in the glass in the longitudinal direction Thermally induced residual stress in the glass 
in the transverse direction

Yield stress of aluminum

Applied traction in the normal and two interfacial shear directions

Angular measurement 


\section{Epigraph}

A definition of friendship:

Pythagoras once wrote: "A friend is the other I, such as are 220 and 284." Such number pairs are called "amicable". To illustrate, the proper divisors (ie. divisors other than the number itself) of the number 220 are as follows:

$1,2,4,5,10,11,20,22,44,55,110$

The proper divisors of the number 284 are as follows:

$1,2,4,71,142$

The sum of the proper divisors of the number 220 is:

$1+2+4+5+10+11+20+22+44+55+110=\underline{284}$

The sum of the proper divisors of the number 284 is:

$1+2+4+71+142=\underline{220}$

...the relationship is clear. I cannot fathom the possibility of a definition of friendship finer than this. The aim of the body of work presented herein, as is the case for all research, is to illustrate and illuminate the relationships, connections and couplings between seemingly dissimilar things; phenomena which can be deemed "amicable" if you will. Sometimes these friendships are obvious; oft they are not. Through unwavering resolve and tenacity though, they will reveal themselves; eventually. In the words of Johann Sebastian Bach, written in an inscription accompanying the final two cannons before the trio sonata in The Musical Offering: Quaerendo Inventis - Seek, and ye shall find.

P.P.K. 2009 


\section{Chapter One: Introduction and Literature Review}

Design against fracture is an issue of paramount importance to the design engineer in virtually every field and industry involving structures experiencing variable loadings. Of these fields, the aerospace industry is particularly sensitive to the understanding and prediction of failure in order to ensure proper design of aerospace structures and ultimately, to preserve the safety of passengers and other personnel.

Since the introduction of modern jet transport in the 1950's, a revolution not only in travel has occurred, but a revolution in design as well. As larger aircraft were being built previous design principles had to be modified to accommodate for a growing risk of inflight safety. In-service failures of large commercial aircraft rapidly altered the focus of design from a strictly performance based point of view to a perspective governed largely by safety.

Pressurized fuselages with larger diameters have significantly increased the loads in the fuselage material and therein the need for a damage tolerant material with qualified maintenance is born.

The remainder of this thesis discusses one particular solution to the damage tolerance problem: fibre metal laminates (referred to as FMLs herein). FMLs are a class of hybrid 
composite material consisting of alternating layers of thin, high-strength metallic alloys, laminated with unidirectional fibres that are pre-impregnated in an epoxy matrix prior to curing. By virtue of this consolidation of both monolithic metal and fibrous composite, FMLs exhibit many desirable effects. Formability and plasticity prior to failure are maintained through retention of their metallic constituents, while reduced density, damage tolerance and directional properties arise through the implementation of fibrous composites.

The focus of the thesis deals with the mechanical behaviour of FMLs in response to a pin bearing load with respect to the current literature and several results and insights into this behaviour will be presented. A practical methodology for both modeling and testing progressive damage in mechanically fastened FML joints is also presented and applied to examine the pin bearing response and subsequent failure of FMLs.

\subsection{Historic Development of FML Technology}

The concept of bonding metallic sheets together to create a laminated structure was first developed and introduced by de Havilland; an English aircraft company in the 1940's [1]. It was quickly discovered that the rate of crack growth in fatigue induced cracks was reduced since surface cracks could be effectively bridged by the underlying, un-cracked metallic layers [2-7]. This bridging would cause a transfer of load to the un-cracked metallic layers, significantly reducing the stress intensity factor at the crack tip, thus prolonging the fatigue life of the structure [4]. In addition, as a consequence of the fact that the metallic layers in the laminate are thin, the amount of constraint due to plasticity 
at the crack tip is reduced and the conditions surrounding the crack tip are that of plane stress [8-10]. These two factors, ultimately lead to an exhibited increase in fracture toughness for laminated plates (as high an increase as a factor of 2) when compared to their monolithic counterparts $[3,4]$.

Eventually, this notion was brought to the Technical University of Delft (TUDelft) in the Netherlands where Professor Boud Vogelsang was also investigating adhesive bonding on aluminum structures. Emphasis on lighter structures for aerospace applications was on the forefront of design at the time and these built-up, metallic structures were seen as an excellent way to reduce weight. At the same time, laminated composite materials were already beginning to receive praise for their exceptional strength to weight ratio (among other material properties) and therefore it only seemed logical to introduce composite fibres into the laminated structure to further improve the desired result and bridging effect: thus FMLs were born.

Several combinations of monolithic metals and composite fibre systems were investigated including initial considerations of a carbon-aluminum laminate (CARALL) and a graphite-titanium laminate (TiGr) [11]. The relatively low corrosion resistance and high moisture absorption of carbon fibres within CARALL were quickly identified as deleterious to the overall performance of the laminate and further development was postponed. Of the many configurations of metal and fibre initially examined, it was found that high strength 7075-T6 and 2024-T3 aluminum, coupled with high modulus aramid fibres resulted in an ultra-light, damage tolerant material. This Aramid Reinforced Aluminum Laminate (dubbed ARALL) seemed quite promising and application to 
aircraft wings, specifically the Fokker F-27 aircraft, began in earnest in the 1980's [12$16]$.

In the preliminary applications of ARALL to aircraft structures, it was discovered that ARALL was sensitive to significant reductions in blunt notch strength as a result of the drilled holes necessary to rivet a panel together to form the skin of a wing [17]. This represents the first identification of the overall sensitivity of FMLs in response to a bearing load configuration. Albeit sensitive to blunt notch strength reductions (which could be counteracted via the implementation of doublers and stiffeners), a weight savings of $33 \%$ versus the original monolithic aluminum structure, coupled with a fatigue life on the order of three times the design life of an F-27 made the employment of ARALL in aircraft wings an undeniable success [1].

The newest generation of FMLs is a GLass REinforced (GLARETM) FML, capitalizing on the superior fibre/epoxy adhesion and high compressive strength of the glass fibres when compared to aramid fibres [17-19]. GLARE is well suited to fuselage applications simply because it is not prone to the low frequency fibre failure characteristic of ARALL and the inertness of the glass fibres, coupled with low moisture absorption makes it more robust environmentally speaking.

The application of GLARE in aircraft is not limited to the aft fuselage sections. It has been proposed that as much as $30 \%$ of the aircraft's primary structure can be made of GLARE including longerons, stringers, ailerons, wings and frames [19]. The application of GLARE panels in the upper fuselage of the Airbus A380 commercial aircraft represents the first use of FMLs within a large scale commercial aircraft and is an indication of the future applications of GLARE within modern airplanes. However, as 
with the application of any new material, a detailed examination of the mechanical behaviour and constitutive nature of GLARE, in response to numerous loading configurations is required before implementation into new and existing aircraft designs can be performed with confidence.

\subsection{Constituent Materials and Mechanical Behaviour of FMLs}

\subsubsection{Constituent Materials and Manufacturing Process}

GLARE is composed of three constituents: monolithic aluminum (either 7075-T6 or 2024-T3); high-strength $\mathrm{S}_{2}$-glass fibres and an FM-94 epoxy adhesive system. The laminate is formed by alternately laying sheets of aluminum with nominal thickness varying from $0.2 \mathrm{~mm}$ to $0.5 \mathrm{~mm}$ and unidirectional layers of pre-impregnated glass fibres (prepreg) with a nominal thickness of $0.125 \mathrm{~mm}$. Figure 1.1 depicts a typical FML layup. The unidirectional fibre layers can be laid in tandem in a cross-ply fashion to give strength in both the longitudinal direction (rolling direction of the aluminum) and the transverse direction $\left(90^{\circ}\right.$ to the rolling direction).

To improve the shear strength of the laminate, unidirectional prepreg layers can also be laid in a $\pm 45^{\circ}$ fashion. This alternating process is repeated until the desired thickness of the laminate is achieved. This consolidation of metal and fibrous composite creates a structure, not a material and therefore GLARE is not truly metallic yet it is not a composite either; it is a hybrid composite possessing properties of each constituent and therefore analysis of its behaviour requires special attention and approaches. 


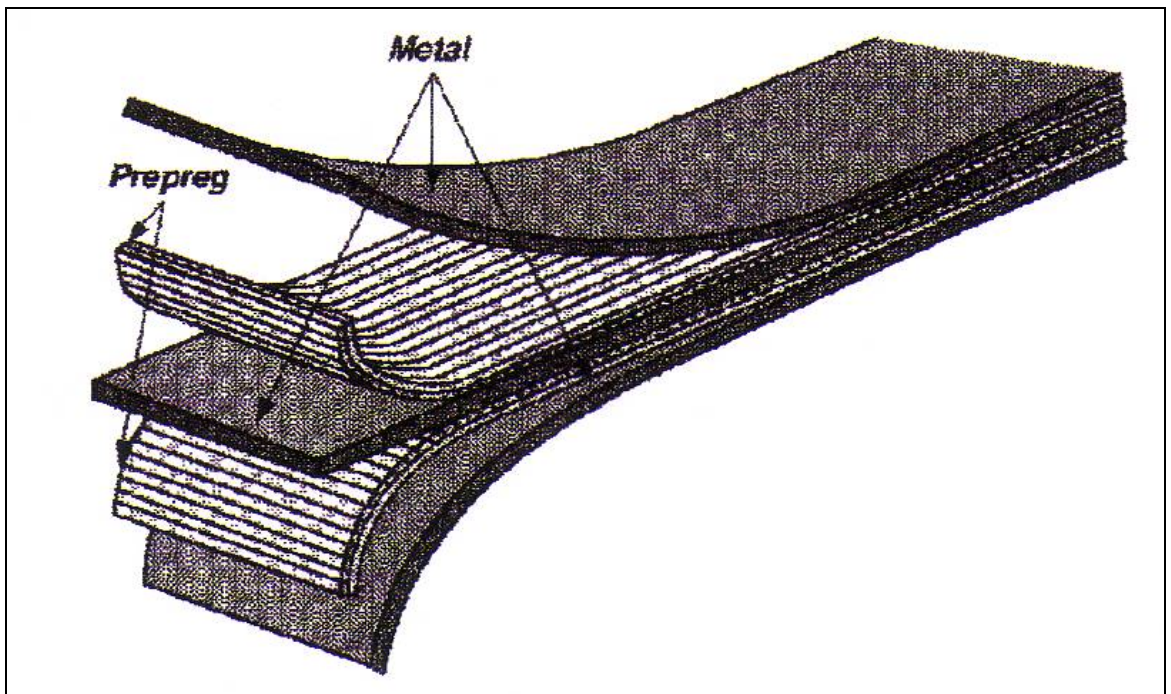

\section{Figure 1.1: A typical FML lay-up depicting both the metallic and fibrous layers (adapted from [20]).}

Six variations of GLARE exist to date forming its own sub-family within the FMLs. Each GLARE variant is tailored to different applications and material advantages via specific orientations of the fibre layers and volume of aluminum within the laminate. Table 1.1 summarizes the commercially available GLARE variants and their constituents.

Not unlike standard composite laminates, quasi-composites like FMLs also follow a convention identifying their lay-up and variant. The standard practice for identifying the lay-up is explained in the following example. A GLARE3 lay-up with three layers of 2024-T3 aluminum and two layers of prepreg would be identified as:

[2024-T3 / $0^{\circ}$ glass $/ 90^{\circ}$ glass / 2024-T3 / $0^{\circ}$ glass $/ 90^{\circ}$ glass / 2024-T3]

which is the same variant as shown in Figure 1.1. The nomenclature for correctly identifying this laminate is as follows: GLARE3-3/2-0.3; which is read as a GLARE variant 3 (implying two unidirectional prepreg plies laid in a cross-ply fashion per prepreg layer) with three layers of aluminum and two layers of prepreg. The final term in 
Table 1.1: A summary of the commercially available GLARE variants and their constituent materials.

\begin{tabular}{|c|c|c|c|c|}
\hline \multirow{2}{*}{$\begin{array}{c}\text { GLARE } \\
\text { Variant }\end{array}$} & \multicolumn{2}{|c|}{ Aluminum Layers } & \multicolumn{2}{c|}{ Fibre Layers } \\
\cline { 2 - 5 } & Alloy & $\begin{array}{c}\text { Nominal } \\
\text { Thickness (mm) }\end{array}$ & Orientation & $\begin{array}{c}\text { Thickness per Prepreg } \\
\text { Layer (mm) }\end{array}$ \\
\hline GLARE 1 & $7075-\mathrm{T} 6$ & $0.3-0.4$ & Unidirectional & 0.25 \\
\hline GLARE 2 & $2024-\mathrm{T} 3$ & $0.2-0.5$ & Unidirectional & 0.25 \\
\hline GLARE 3 & $2024-\mathrm{T} 3$ & $0.2-0.5$ & Cross-ply (50-50) & 0.25 \\
\hline GLARE 4 & $2024-\mathrm{T} 3$ & $0.2-0.5$ & Cross-ply (67-33) & 0.375 \\
\hline GLARE 5 & $2024-\mathrm{T} 3$ & $0.2-0.5$ & Cross-ply (50-50) & 0.5 \\
\hline
\end{tabular}

the nomenclature identifies the nominal thickness of each aluminum layer, though it is commonly omitted. In this case it is $0.3 \mathrm{~mm}$ although it could have been anywhere from $0.2 \mathrm{~mm}$ to $0.5 \mathrm{~mm}$.

Irrespective of the GLARE variant employed, there is always a minimum $8 \%$ savings in weight when compared to an equivalent monolithic aluminum structure, but this weight savings can be increased to as much as 25 to $30 \%$ [21].

Before the thin sheets of aluminum alloy and the prepreg layers can be bonded together, there are several intermediate steps required to prepare the constituent surface substrates for their final arrangement as a laminate. As a consequence of some of these intermediate steps, there exist both beneficial and deleterious side effects. Details of the precise steps and procedures employed in the preparation of both the aluminum and glass prepreg layers can be easily found in the literature $[21,22]$. Once both the aluminum and the fibre 
layers are prepared, the constituents are now ready for lamination. After a lay-up has been chosen and executed, the complete laminate is cured at approximately 120 degrees Celsius in an autoclave.

The FM-94 epoxy adhesive system which serves as the matrix for the $\mathrm{S}_{2}$-glass fibres is also the adhesive used for laminate bonding during curing in the autoclave. The adhesive matrix used in the fibrous layers contributes significantly to the overall performance of the laminate [21]. It is the adhesive's shear strength which determines the overall bond integrity between the metallic and fibrous layers and is the governing factor in the delamination mechanism, vital to the fibre bridging mechanism present in all FMLs [2325]. The adhesion between it and the corresponding glass fibres is so strong that it often remains intact until cohesive failure [26].

As a consequence of this heterogeneous constituency and of the manufacturing process itself, several distinct properties emerge from FMLs including thermally induced residual stresses, which will be discussed in subsequent sections.

\subsubsection{Mechanical Behaviour}

The material properties of GLARE FMLs are interesting in that, as their name suggests, they exhibit both fibrous and metallic mechanical behaviour. The result is a complex set of properties that have been studied yet require more investigation in the future. The following section will outline GLARE's tensile and compressive behaviour and discuss its advantage or disadvantage when compared to monolithic aluminum structures. The stress-strain curve for GLARE is a hybrid fibre-metal profile depending heavily on the orientation of the majority of the fibre layers and on the loading direction. In general, 
the laminate exhibits an inelastic behaviour under tensile loading due to the plasticity of the aluminum layers and the developed residual stresses which occur as a bi-product of the difference in the thermal expansion of its constituents during curing [21,27]. It has also been observed that irrespective of variant, all GLARE laminates exhibit a lower modulus of elasticity due to the low modulus glass fibres (as a side note, ARALL has a much higher modulus than that of GLARE due to its extremely high modulus aramid fibre constituent) [21]. As a consequence of the difference between the modulus of aluminum and glass fibre, the tensile yield strength of the laminate will also be slightly lower since the proportionately stiffer aluminum layers will attract more stress (by virtue of Hookes' law) and yield sooner than monolithic aluminum under the same loading conditions.

The directionality of the properties of GLARE is a direct consequence of the orientation of the fibre layers. The fibre layers contribute to both stiffness and strength in the direction of their orientation. As a consequence, the strength of GLARE laminates is significantly higher than that of monolithic aluminum in the longitudinal direction of the laminate. In a betraying fashion, in the transverse direction, it is the metal layers and the metal layers alone which govern the strength of the laminate. Thus, when compared to monolithic aluminum, a unidirectional GLARE laminate is weaker in the transverse direction, perpendicular to the fibre orientation. The introduction of cross-ply laminates gave the laminate equal properties in both longitudinal and transverse directions and therefore in a quasi-isotropic sense and irrespective of the direction, the laminate could now possess a higher ultimate tensile strength than monolithic aluminum. 
The compressive behaviour of GLARE is similar to its tensile behaviour in both the magnitude of its specific properties and its sensitivity to fibre orientation. Specifically, the elastic moduli for various GLARE variants are almost equal in magnitude to their tensile counterparts since the glass fibres have a nearly equal contribution to the elastic modulus in both tension and compression. It should be noted though that the compressive yield strength of all GLARE variants is somewhat lower than its corresponding tensile yield strength, but this does not limit GLARE to tension dominated loading applications like its aramid based predecessor, ARALL [21]. Compared to monolithic aluminum though, the compressive yield strength of GLARE is higher in the longitudinal direction but lower in the transverse direction. Table 1.2 summarizes the mechanical behaviour of several GLARE variants in both tension and compression where $L$ represents the longitudinal direction and $T$ the transverse direction respectively.

The stress-strain response of GLARE FMLs is largely governed by the elastic-plastic behaviour of the aluminum layers. The prepreg layers, in general, behave linearly elastic up until their point of fracture with little to no plasticity observed, which is common among fibre reinforced polymers. The overall response of the laminate cannot, therefore, be modeled under elastic analyses alone. Several analytical models predicting the stressstrain response of GLARE have been developed by Hagenbeek [27], Verolme [30] and Van Rijn [31] based upon the mechanical response of the constituents and a rule of mixtures. All three constitutive models have incorporated elastic properties for the aluminum layers (material properties of which are well documented) as well as a Ramberg-Osgood approximation. 
Table 1.2: A summary of some of the static properties of several GLARE variants (adapted from $[21,28,29]$ )

\begin{tabular}{|c|c|c|c|c|c|c|c|c|c|c|c|c|}
\hline \multirow{3}{*}{$\begin{array}{l}\text { Laminate } \\
\text { G: GLARE } \\
\text { A: ARALL }\end{array}$} & \multicolumn{4}{|c|}{$\begin{array}{c}\text { Elastic Modulus } \\
\text { (GPa) }\end{array}$} & \multicolumn{4}{|c|}{$\begin{array}{c}0.2 \% \text { Yield Strength } \\
\text { (MPa) }\end{array}$} & \multirow{2}{*}{\multicolumn{2}{|c|}{$\begin{array}{c}\text { Ultimate Strength } \\
\text { (MPa) } \\
\text { Tensile }\end{array}$}} & \multirow{2}{*}{\multicolumn{2}{|c|}{$\begin{array}{c}\begin{array}{c}\text { Ultimate Strain } \\
\text { (\%) }\end{array} \\
\text { Tensile }\end{array}$}} \\
\hline & \multicolumn{2}{|c|}{ Ten. } & \multicolumn{2}{|c|}{ Comp. } & \multicolumn{2}{|c|}{ Ten. } & \multicolumn{2}{|c|}{ Comp. } & & & & \\
\hline & $L$ & $T$ & $L$ & $T$ & $L$ & $T$ & $L$ & $T$ & $L$ & $T$ & $L$ & $T$ \\
\hline G1 & 65 & 50 & 67 & 51 & 545 & 333 & 424 & 403 & - & - & - & - \\
\hline $\mathrm{G} 2$ & 66 & 50 & 67 & 52 & 360 & 228 & 414 & 236 & 1213 & 317 & 4.7 & 10.8 \\
\hline G3 & 58 & 58 & 60 & 60 & 305 & 283 & 309 & 306 & 745 & 745 & 4.7 & 4.7 \\
\hline G4 & 57 & 50 & 60 & 54 & 352 & 255 & 365 & 285 & 1027 & 607 & 4.7 & 4.7 \\
\hline G5 & 70 & 70 & - & - & - & - & 283 & 283 & 683 & 683 & - & - \\
\hline A2 & 66 & 53 & 65 & 53 & 366 & 228 & 255 & 234 & 717 & 317 & 2.5 & 12.7 \\
\hline A3 & 66 & 49 & 64 & 50 & 607 & 331 & 345 & 360 & 821 & 780 & 2.2 & 8.6 \\
\hline
\end{tabular}

The post yielding portion of the curve is assumed to be perfectly plastic for the aluminum layers and dependant upon the stiffness of the prepreg layer, resulting in a bilinear stressstrain curve [27]. This idealized approximation is obtained by extending the linear plastic part in the strain hardening portion of the curve until it intersects the linear elastic regime of the curve, pre-yield [27]. The intersection of these two lines, the so-called "kneepoint", allows the formation of the bilinear approximation.

Inherent to the prediction of tensile properties is a modified rule of mixtures taken from composites and applied to FMLs known as the "Metal Volume Fraction" (MVF) approach $[32,33]$. By definition, the MVF is defined as the ratio of the sum of thicknesses of the individual aluminum layers to the total laminate thickness [33]. An expression for 
the MVF is given in Equation (1.1) where $t_{a l}$ is the thickness of each individual aluminum layer, $t_{\text {lam }}$ is the total thickness of the GLARE laminate and $p$ is the number of aluminum layers.

$$
M V F=\frac{\sum_{1}^{p} t_{a l}}{t_{\text {lam }}}
$$

If we examine Equation (1.1), we see that a MVF of 1.0 designates a pure, monolithic aluminum structure. Conversely, an MVF of 0.0 designates a structure composed purely of the glass fibre prepreg. A linear relationship is assumed to exist between the two extrema requiring extrapolation from averaged tested laminate data to create the theoretical MVF $=0.0$ solution [33]. The MVF is specific to both the variant and employed lay-up. For example, a typical GLARE3-3/2-0.4 variant would exhibit an MVF of 0.705 . Thus, even though it is a hybrid composite, GLARE is still mostly aluminum. With the MVF for a specific variant and lay-up of GLARE established, it is now possible to calculate a particular laminate property such as ultimate tensile strength or tensile yield strength in a smeared fashion via Equation (1.2):

Laminate Property = MVF x "Metallic Property" + (1 - MVF $)$ x "Fibre Property"

Calculating properties such as tensile, compressive, shear and blunt notch strengths in this manner has been deemed valid by comparison to experimentally derived values for $0.45<\mathrm{MVF}<0.85[33]$.

Aside from the aforementioned tensile and compressive properties, GLARE exhibits numerous material advantages over conventional monolithic metals and several 
investigations aimed at understanding GLARE in this capacity can be found in the literature including: in-plane shear [16,32,34]; fatigue and fracture [35-42]; residual strength, damage tolerance and fracture toughness [43,44]; blunt notch strength [45-47]; impact resistance [48-51]; burn through resistance and lightning strike [52-54] and corrosion resistance $[21,55]$.

\subsection{The Bearing Behaviour of FMLs}

In all aerospace applications, irrespective of the material system employed, the associated bearing strength of a material is a principle design parameter for preventing failure of mechanical fasteners and their consequent joints, which to this day are still the primary means of structural fastening. A review of the literature regarding the development of both experimental and analytical techniques was conducted in an effort to steer the development of the test protocols, analysis and practical methodology presented herein. Although some investigations have been conducted on the bearing strength of FMLs [5664] in both pin and bolt loading, there is a notable deficit in the literature. The focus of the above methodologies has largely been on the generation of bearing ultimate strength values rather than an examination of the failure mechanism and/or the associated strain field near the fastener hole. In addition, the literature is all but void of any mention of the notion of characteristic dimensions, commonly employed in composite joint design [6567]. With aircraft still fastened primarily by mechanical means, understanding the bearing behaviour of FMLs in full capacity - not just their ultimate bearing strength - 
towards developing a practical damage prediction methodology for a new aircraft material is of topical importance.

In general, investigations into the bearing behaviour of FMLs can be categorized into two main streams: experimental and analytical. While some studies have considered both of these categories, most have emphasized the empirical approach with their methodologies conducted in accordance with ASTM D953 [68] and ASTM E238 [69] with predetermined definitions of both bearing yield and ultimate bearing strength. Slagter [60] performed experimental investigations on the pin and bolt bearing behaviour of both ARALL and GLARE laminates. Emphasis was placed on testing configurations and geometries which would ensure the generation of bearing strength data for only a bearing failure mode and as a consequence, net tension and shear-out modes of failure were not observed. This is not necessarily an oversight since, in terms of prudent design, failure in mechanically fastened aerospace structures is encouraged to occur in a bearing mode only since the remaining two modes cannot sustain any additional load once the joint has collapsed. Data was generated for both pin and bolt bearing testing configurations and it was observed that laterally restrained, finger tightened specimens exhibited a considerable increase in strength near 20\% [60]. This increase in strength did not increase proportionately with an increased clamping force supplied by a torqued bolt and it was attributed to a restriction of layer delamination and buckling from the presence of the added boundary condition provided by the lateral restraint. In a pin bearing configuration, it was qualitatively observed that delamination buckling of the aluminum layers preceded joint collapse and it was loosely proposed that this phenomenon was the mode of failure eliciting the collapse of the joint. 
An analytical model was also developed concurrently during the experimental portion of the investigation by Slagter [60]. Based upon the shearing/extensional energy of the fibre layers, this analytical model was compared to the experimental findings with satisfactory agreement but it represented a departure from the qualitatively observed experimental findings: that the governing mode of failure was delamination buckling of the aluminum layers rather than the composite fibre prepregs.

Further parametric studies of the bearing strength of FMLs were performed by Wu and Slagter [61]. In this experimental study, the sensitivity of both pin and bolt bearing to specific geometries such as the edge distance to diameter ratio (E/D) and the width to diameter ratio (W/D) was examined. Investigated E/D ratios included 1.5, 2, 3 and 5 while W/D ratios (with E/D fixed at 3) included 2, 4, 6 and 8. The resulting failure mode was found to be sensitive to the variation of these parameters and it was concluded that a minimum $\mathrm{E} / \mathrm{D}=3$ and $\mathrm{W} / \mathrm{D}=4$ enabled the full development of the bearing failure mode [61]. This clearly identifies that $\mathrm{E} / \mathrm{D}=3$ represents a critical ratio for bearing strength and should be the geometric parameter of interest in a practical damage prediction methodology. Caprino et al. [62] revisited Slagter's [60] pin and bolt bearing investigations and also examined the effects of specimen geometry on failure mode (net tension, shear-out and bearing) by varying the same two geometric parameters, (W/D and E/D) and generated bearing strength data. The employed range of E/D ratios included several with decimal values such as $1.6,3.8$ and 4.3. While their results did not explicitly state what the critical $\mathrm{E} / \mathrm{D}$ ratio was, they do conclude that the presence of the aluminum alloy does allow for lower ratios which preserve the true bearing mode of failure. Post-testing optical 
microscopy of some of the failed specimens revealed extensive delamination and buckling of the aluminum layers supporting the notion of the laminate's bearing strength dependence on delamination and buckling.

Meola et al. [63] conducted pin bearing tests on GLARE FMLs identifying that the pin bearing test configuration is the most critical one for the simulation of mechanical joint behaviour since the lack of lateral restraint yields the most conservative value of bearing strength. Tests were conducted in accordance with ASTM-D953 [68] save for the definition of ultimate bearing strength in which it was decided to record the maximum load sustained by the specimen as the ultimate bearing strength rather than retaining the $4 \%$ permanent hole deformation stipulated by the standard. Numerous geometrical configurations were tested analogous to the work of Caprino et al. [62] focusing on the generation of bearing strength data for all possible failure modes. The inclusion of lock-in thermography as a means of non-destructive evaluation was particularly noteworthy since it allowed for in situ observation of delamination occurring between the aluminum and glass layers.

Finally, the authors also conclude that in a bearing failure mode, the ultimate pin bearing strength of GLARE attains a constant value near $588 \mathrm{MPa}$ and is fully independent of fastener hole diameter [63]. This result is counterintuitive since, by virtue of its definition, the calculation of bearing strength is inversely dependant on the diameter of the hole as expressed in Equation (1.3)

$$
\sigma_{b}=\frac{P}{D t}
$$


In addition, the independence of bearing strength on fastener diameter is a questionable conclusion since it has been observed experimentally, in the same study, that delamination serves as a precursor to joint collapse. The onset and propagation of delamination, in this loading configuration, is invariably influenced by the magnitude of the stress concentration generated at the circular notch root. Although numerous mechanisms of failure were indeed examined and valuable ultimate bearing strength values were found, no real insight was gleaned into the actual behaviour of the joint in response to a pin bearing loading configuration.

Van Rooijen et al. [58] conducted a series of finger tightened, bolt bearing experiments on a GLARE 2 FML, also varying E/D and W/D parameters. Profiles of the calculated bearing stress for different E/Ds versus pin displacement as measured, in a global sense, by an attached extensometer were generated and it was abundantly clear that an $\mathrm{E} / \mathrm{D}=3$ generated the most favourable results (highest sustained load) in a bearing failure mode. Finite element (FE) models were generated to accompany the experimental results gathered with good agreement, though any investigation into delamination - previously identified as the interlaminar separation between the fibre and metal layers present before joint collapse - was not included in the models since it required a local loss of interaction between adjacent plies and the implementation of interface elements [58]. Although the concept of failure prediction in hybrid-composite joints via FE analysis and characteristic dimensions was briefly mentioned, any further investigation was dismissed. Most recently Frizzell et al. [56], conducted pin bearing experiments on a GLARE3-3/20.4 laminate to identify which E/D and W/D ratios correspond to each failure mode though no specific adherence to an ASTM standard was reported. They identified that 
very low ratios $(\mathrm{E} / \mathrm{D}=1.3$ and $\mathrm{W} / \mathrm{D}=2.0)$ were required to induce shear-out and net tension failure modes but employed a very high $\mathrm{E} / \mathrm{D}=6$ for their bearing failure specimens. It has been shown in previous studies $[58,61,62]$ that a high $\mathrm{E} / \mathrm{D}=6$ is very conservative and not the critical case for design. Employing such a high ratio in design would introduce much additional material into joints making the desired structure inefficient in terms of weight.

The authors in [56] then examined failed specimens using scanning electron microscope (SEM) imaging through various percentages of their ultimate failure load. The imaging revealed that in the high E/D bearing specimens, final failure was accompanied by the complete separation of aluminum layers from the glass and was identified as an unstable process when the experiment was performed under load control.

In general, there exists no practical damage prediction methodology for the pin bearing behaviour of FMLs, centred on the critical design geometries and incorporating an investigation of the associated strain field near the fastener hole. It is this deficit in the literature, coupled with the cited works that prompted the investigation discussed in the present study. 


\subsection{Failure Prediction and Modeling of FMLs}

\subsubsection{The Characteristic Dimension Method}

The analysis of the induced stress field and prediction of failure in both elastically isotropic and orthotropic plates as the result of a load applied through a mechanical fastener has been previously investigated [70-79].

Chang et al. [80,81] developed the characteristic dimension method for predicting failure in composite joints based on previous studies by Agarwal [82] and Whitney \& Nuismer [83]. It is a very practical, semi-empirical approach that is still used today [65-67]. In the characteristic dimension method, failure in a composite laminate joint has been proposed to occur when the combined stresses in any one of the plies within the laminate fulfills a selected failure criterion at any single point along an artificial characteristic curve [80]. The curve is generated by first determining the characteristic dimensions, namely the tensile and compressive characteristic dimensions. The values for tensile characteristic dimension (TCD) and compressive characteristic dimension (CCD) are derived from open-hole, blunt notch strength and bearing strength tests, respectively. Figure 1.2 and Figure 1.3 depict schematic representations outlining the concept behind the characteristic dimensions.

Conducting a FE analysis using the same geometry as the tested configuration enables the plotting of the distribution of stresses around the stress concentration. More specifically, one can plot the stress as a function of increasing distance moving away from the fastener 


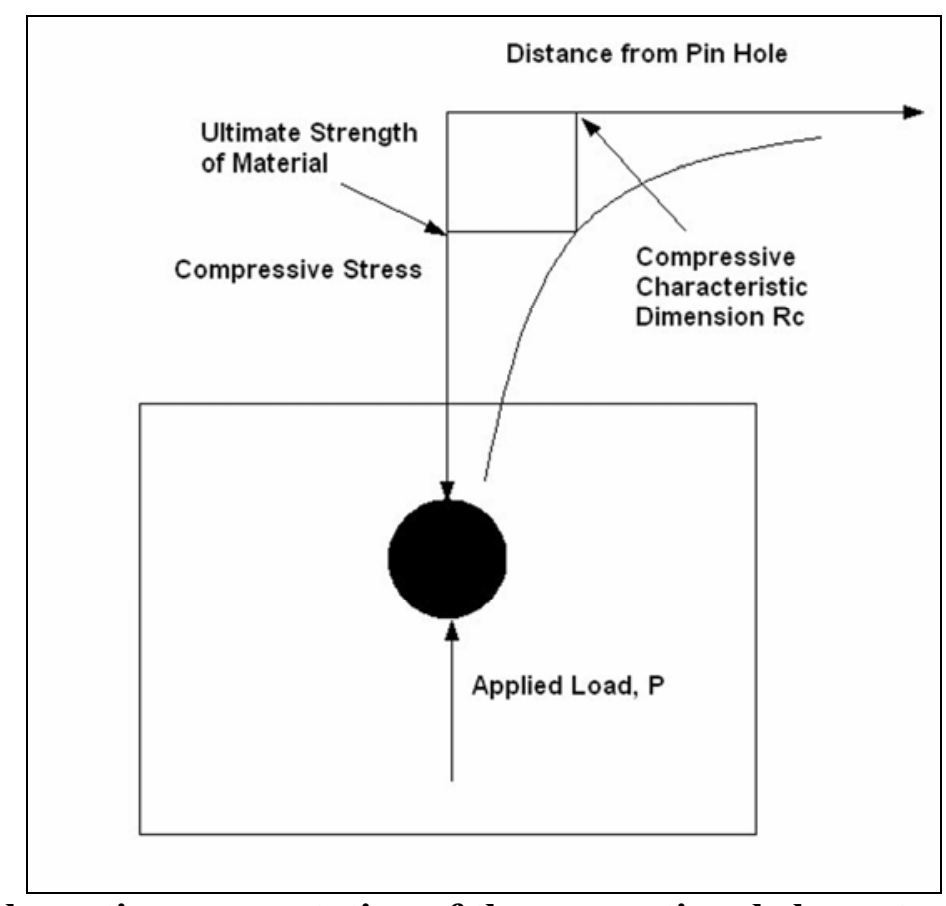

Figure 1.2: Schematic representation of the conventional characteristic dimension method for the compressive characteristic dimension.

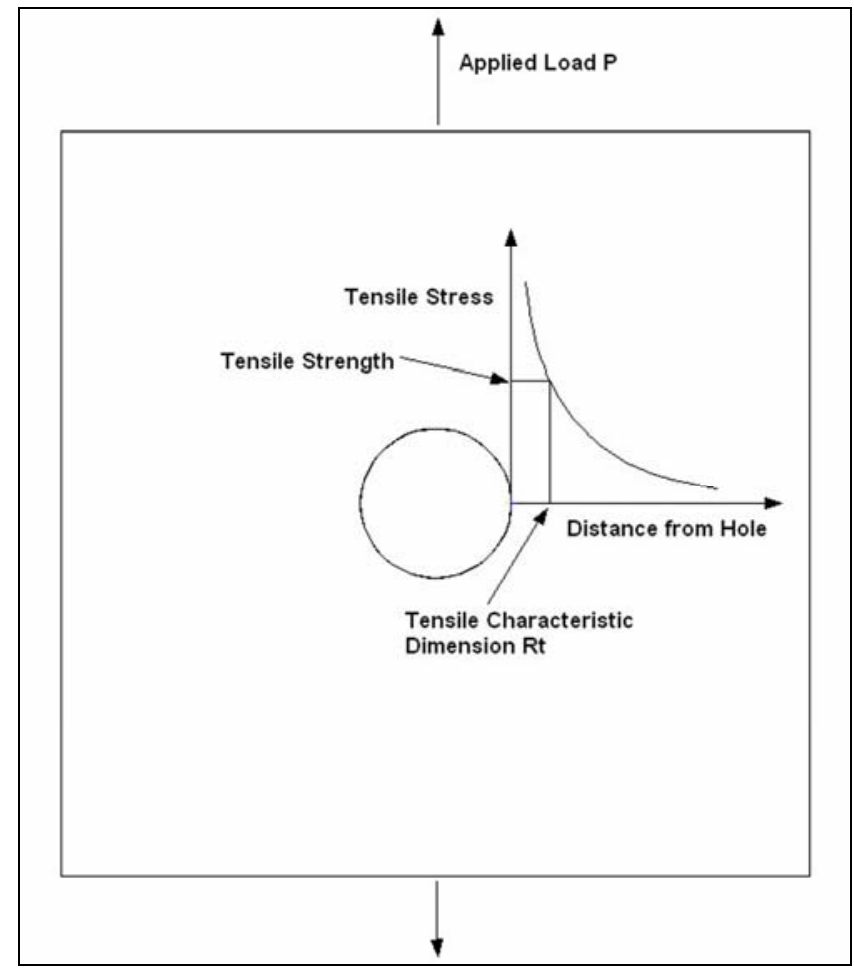

Figure 1.3: Schematic representation of the conventional characteristic dimension method for the tensile characteristic dimension. 
hole. By definition, the CCD is the distance from the leading edge of the fastener hole (in the direction of loading) to a point in the profile where the compressive stress equals the ultimate strength of the laminate. In contrast, the TCD is defined as the distance from the peripheral edge of the open hole to a point in the stress profile where the tensile stress equals the ultimate tensile strength of the material. Once the CCD and TCD have been determined in this manner, the characteristic curve can be constructed using Equation (1.4) as proposed by Chang et al. [80]:

$$
r_{c}(\theta)=\frac{D}{2}+R_{t}+\left(R_{c}-R_{t}\right) \cos \theta,-90^{\circ} \leq \theta \leq 90^{\circ}
$$

Here the parameters $R_{c}$ and $R_{t}$ are the CCD and TCD respectively and the angle $\theta$ is measured in a clockwise fashion from the vertical axis as depicted in Figure 1.4. The angle $\theta$ also provides an indication as to the mode of failure which is most likely to occur depending on what value $\theta$ takes. For small values of $\theta\left(-15^{\circ} \leq \theta \leq 15^{\circ}\right)$ failure will most likely occur in the bearing mode; for intermediate values of $\theta\left(30^{\circ} \leq \theta \leq 60^{\circ}\right)$, shear out becomes the dominant mode of failure and for large values of $\theta\left(75^{\circ} \leq \theta \leq 90^{\circ}\right)$, failure is most likely cause by net tension [81].

\subsubsection{FE Modeling of FMLs}

FE modeling of composites has typically employed thin shell elements taking the assumption of plane stress by virtue of the exaggerated difference between their through the thickness dimension and their in-plane dimensions. In 1995, Hashagen et al. [84] developed a solid-like shell element specifically for FMLs. The element was developed 


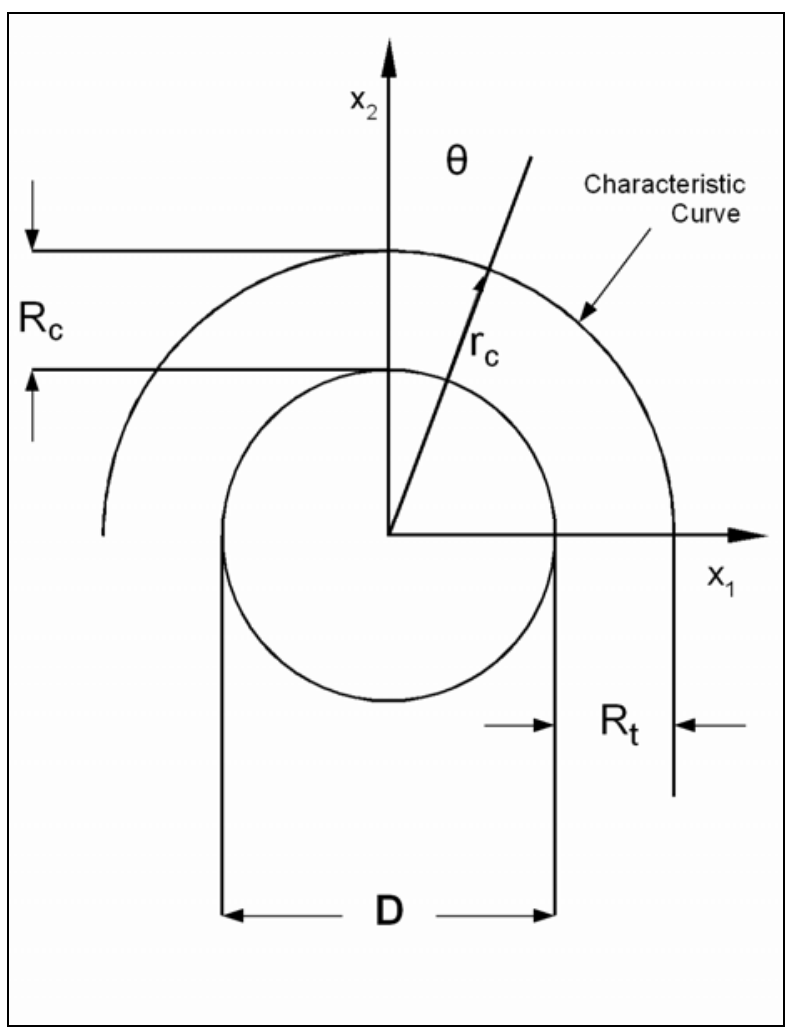

Figure 1.4: Schematic description of the characteristic curve with angle $\theta$ shown.

in response to the fact that modeling with standard hexahedral elements leads to locking for exaggerated aspect ratios yielding erroneous results. Thus an element with sixteen nodes on both the top and bottom surfaces for which only translational degrees of freedom are defined was developed, effectively bridging the gap between solid hexahedral elements and thin shell elements.

The introduction of a solid-like shell element enables the modeling of composite materials at the meso-mechanical scale by allowing each individual layer through the thickness to be modeled by an element.

In a later study, Hashagen et al. [85] conducted a FE investigation of spliced FML panels corroborated by the empirical study performed by de Vries et al. [86]. Due to the previous success, solid-like shell elements were employed once again and now a layer of 
interface elements was inserted in between adjacent layers of aluminum and prepreg in an effort to simulate the resin rich zone present there. Delamination was accounted for in this instance via the introduction of a Hoffman based yield function, however, this approach is limited by its dependency on mode II crack growth only. As a result of this limiting factor, specimens that exhibited any out of plane bending due to through the thickness asymmetries as a result of the splicing process lead to large errors between experiment and simulation [85]. The main factor behind this discrepancy is the sheer fact that out of plane bending introduces failure in mode I in addition to mode II and thus a mixed mode treatment of delamination is relevant.

Remmers and de Borst [87] also implemented the solid-like shell element and modeled FMLs at the same meso-scopic scale while investigating the phenomenon of delamination buckling inherent to all composites under compression. Though the authors consider delamination in the resin rich layer between adjacent plies of aluminum and glass prepreg, they do not consider plasticity in the aluminum or damage in the prepreg layers. In the accompanying experimental investigation, it is reported that experiments with GLARE FMLs exhibit a softening behaviour of the interface that resembles an exponential curve [87]. The analyses were essentially a numerical demonstration of the stability of the interface softening scheme and thus were inherently simple. Most recently, FE studies by Linde et al. [88], Linde and de Boer [89] and van Rooijen et $a l$. [58] on both inter-rivet buckling and bolt bearing included failure in the assumed transversely isotropic glass prepreg layers as well as delamination between adjacent layers. While the results produced agreed satisfactorily with empirical data, none of the 
studies considered residual stress implementation, nor did they include the most recent advances in delamination modeling as applied to the case of pin bearing.

\subsubsection{Delamination Modeling}

Numerous studies on the examination of delamination, both experimental and analytical, have been performed and are available in the literature [90-102]. Newer analyses [87$89,103,104]$ often implement the use of the FE method and interface elements in which the elastic response is governed by a stipulated traction-separation law until the point of damage. The point of damage onset is the satisfaction of an interfacial criterion which may be of the maximum nominal stress/strain variety or of a quadratic nominal stress/strain fashion, among others. Following the satisfaction of the interfacial fracture criterion, the evolution of damage within the interface can be specified in terms of fracture energies in either a tabular form - which would be a direct function of the mode mix - or through a selection of analytical forms. These analytical forms state that failure under mixed mode conditions is governed by an interaction of the fracture energies such that failure will occur in one of the individual modes of failure (the normal or 2 in-plane shear directions) of the interface. The expressions for the forms of these interactions particularly the so-called B-K criterion [105] - along with the accompanying elastic response in terms of a traction-separation law will be shown in Chapter 4 along with their implementation into the practical damage prediction methodology presented herein. In general though, studies by de Moura et al. [106], Camanho et al. [107-109] and Turon et al. [110] have demonstrated that delamination criteria in terms of nominal stress/strains coupled with the aforementioned analytical forms of damage evolution through the 
interaction of fracture energies and mode mix are very suitable for application to the FE method and represent the state of the art in delamination modeling.

\subsection{Thesis Motivation, Objectives and Overview}

\subsubsection{Thesis Motivation}

FMLs have already been implemented in next-generation civilian and military aircraft. The most recent and poignant example is the Airbus A380 aircraft in which portions of the fore and aft regions of the aircraft's upper fuselage are composed of GLARE panels as depicted in Figure 1.5. The designers of the F-35 Joint Strike Fighter are also considering the application of a Graphite Reinforced Titanium Laminate (TiGr, pronounced "tiger") on several parts.

In addition, retrofits and patch repair composed of FMLs are being installed on aircraft already in production in an attempt to extend their lifetimes by virtue of the fatigue insensitivity and damage tolerance of FMLs.

Since the current ideology in aircraft manufacture still relies heavily on the use of mechanically fastened joints as a means of assembling aircraft structures, the need to further understand the behaviour of FMLs, in the capacity of bearing behaviour, is of topical importance and will only increase as such due to their continued application. Therefore, bearing behaviour remains an important avenue of research as current and new FML technologies continue to replace traditional monolithic aluminum in sensitive, primary aircraft structures. 


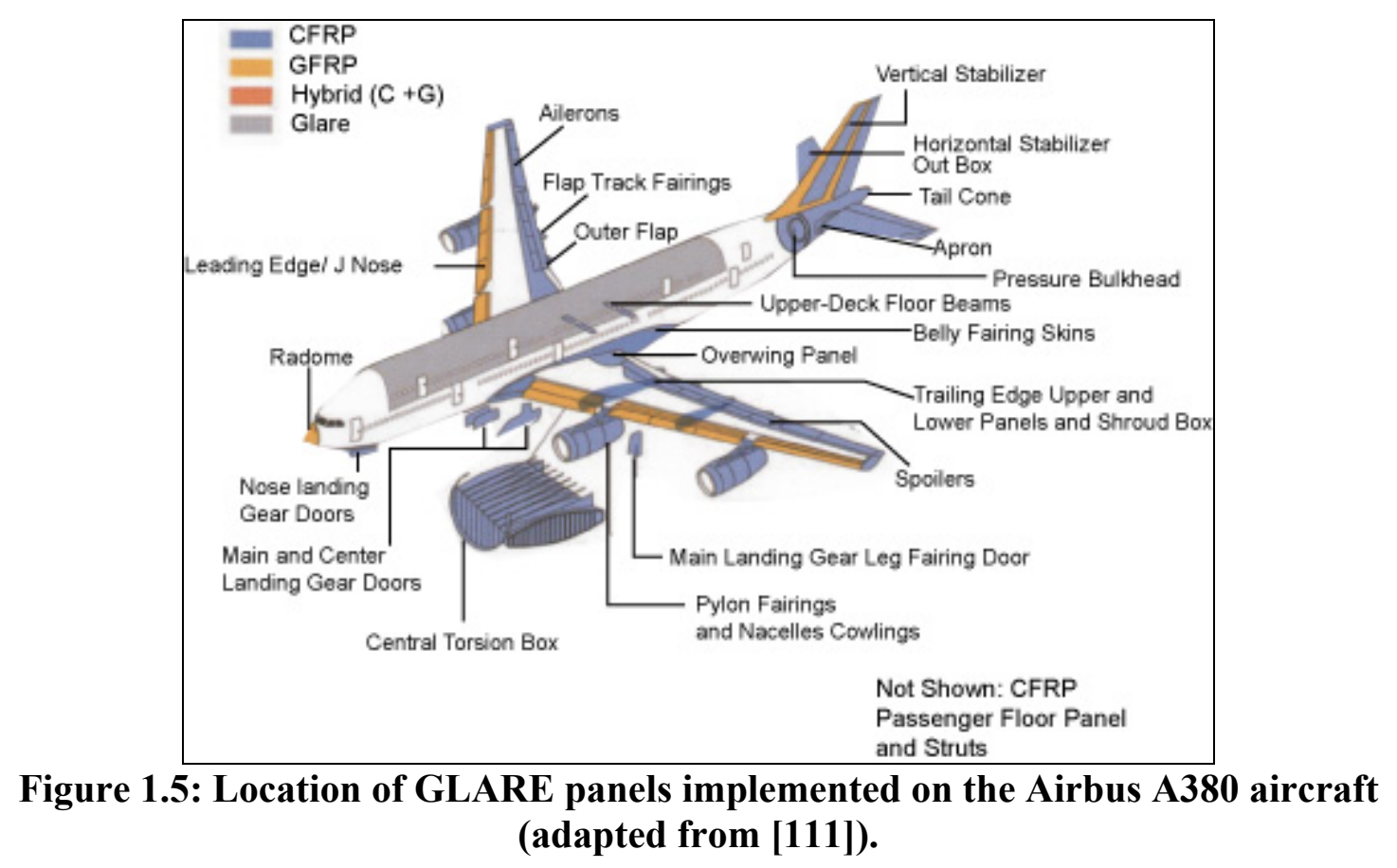

The development of an experimentally investigated, predictive tool as part of a practical methodology would be valuable to the aircraft designer since it would reduce the need for time consuming and costly experimentation. Furthermore, examining the critical case in terms of geometry $(\mathrm{E} / \mathrm{D}=3)$ is important since establishing a progressive damage methodology for this case enables the development of a suitable baseline for use in design.

Finally, with a practical methodology in hand, developers of new FML technologies could streamline and expedite the certification process required for all new aircraft materials, putting into use the state of the art in advanced materials for modern civilian and military aviation. 


\subsubsection{Thesis Objectives}

Bearing in mind the aforementioned research motivations, the objectives of the research described herein are as follows:

- to conduct an in-depth review, from the most recent sources, of the current FML technology and FML testing in a pin bearing configuration;

- to create meso-mechanical level, FE models of GLARE FMLs such that a redefinition of the compressive characteristic dimension can be performed, thus creating a novel application of the approach to hybrid composites;

- to develop an experimental methodology such that an in depth examination of the associated strain field surrounding the fastener hole in a pin bearing loading configuration can be performed in both a global and local measurement scheme;

- to develop a practical pin bearing damage modeling methodology for GLARE to predict the onset and propagation of delamination damage with the previously unconsidered, thermally induced residual stress and if necessary, employ the results from material characterization experiments to support this development;

- to develop a series of pin bearing simulations on GLARE and to compare the results of said simulations with the experimental results in order to support the predictions, thus creating a tool for the analysis and design of mechanically fastened GLARE structures. 


\subsubsection{Thesis Overview}

This thesis presents the results of an experimental, analytical and numerical investigation into the strength of FMLs loaded in pin bearing as part of the development of a practical progressive damage methodology. The remainder of this thesis is thus organized into the following chapters:

- Chapter 2: The modified characteristic dimension approach as applied to GLARE FMLs.

- Chapter 3: Development and results of bearing strength tests as performed on quasi-isotropic and orthotropic GLARE variants and application of a yield definition developed herein to a GLARE tension test.

- Chapter 4: Development of a progressive damage FE model for GLARE in pin bearing.

- Chapter 5: Results, discussion and comparison to the experimental findings of the progressive damage FE model for GLARE in pin bearing.

- Chapter 6: Conclusions drawn from the research.

- Chapter 7: Future considerations and follow up work based on this study.

\subsubsection{Thesis Contributions}

The aim of any body of research and specifically that presented in a thesis is the contribution of knowledge to the field. A summary of the contributions made by the current study are summarized as follows: 
- The characteristic dimension approach commonly employed for composite materials has now been modified to account for the structure of FMLs and subsequently extended to predict their failure.

- The development of a failure mechanism (the YDB mechanism) for GLARE FMLs loaded in pin bearing governed by the coupling of yielding, delamination and buckling.

- The development of an experimental protocol to investigate the predictions of both the modified characteristic dimension approach and the YDB mechanism, incorporating both global and local measurement schemes. This protocol was successfully applied to both quasi-isotropic to anisotropic materials.

- The development of a comprehensive characterization of material response for FMLs loaded in pin bearing and in tension.

- The development of a definition of yield in materials loaded in a pin bearing configuration. This definition has also been extended to the extraction of tensile yield strengths and successfully applied to both quasi-isotropic and anisotropic materials.

- The development of a practical progressive damage FE methodology for simulating and predicting failure of GLARE FMLs loaded in pin bearing. 


\section{Chapter Two: Modified Characteristic Dimension and Failure Prediction}

As mentioned in the literature review, the calculation of the $\mathrm{CCD}$ in a bearing failure mode is based on the ultimate tensile strength of the laminated material in a smeared properties fashion. Application of this methodology directly, without reconsideration and modification, is not possible for GLARE laminates due to their material structure and the qualitatively observed delamination buckling phenomenon. Therefore, to successfully apply the characteristic dimension method to GLARE FMLs, a modified definition of CCD and a new failure mechanism is proposed herein.

\subsection{Definition of a Modified CCD and Proposed Yielding Through Delamination Buckling}

The revised definition of the CCD, simply stated, is as follows: on a plot of compressive stress versus distance from fastener hole, the modified CCD is defined as the distance moving away from the leading edge of the fastener hole (in the direction of loading) to a point in the stress profile where the compressive stress equals the yield strength of the aluminum layer. Figure 2.1 is a schematic representation of this modified definition. 


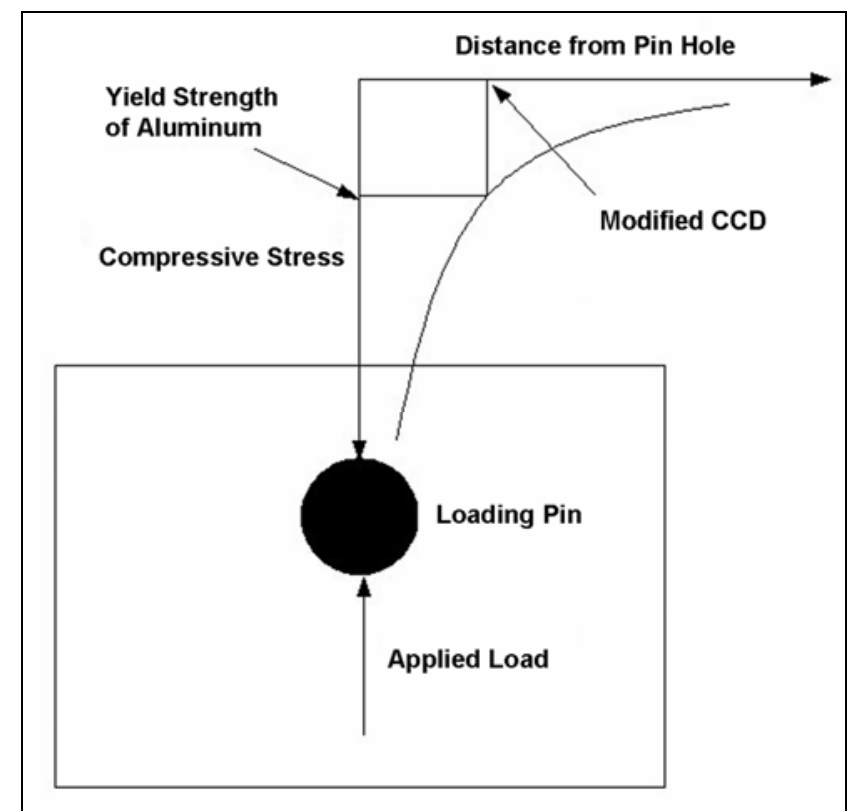

Figure 2.1: Schematic representation of the modified CCD.

The rationale behind this new definition stems from the existing and proposed notions resulting in a new failure mechanism.

It has been observed previously that in a bearing failure mode, during loading, delamination between the aluminum layers and the prepreg layers begins and reaches a critical de-bonding length [60]. What can be drawn from this is that since the yielding of aluminum determines the transfer of load to the glass and thus the amount of delamination, it is a critical parameter. However, since the developed de-bonded area leaves the glass prepreg plies laterally unsupported, they too will buckle along with the aluminum layers and implies a connection between them.

Thus, a new failure mechanism can be proposed herein which shall be tentatively called the Yielding Through Delamination Buckling (YDB) mechanism. The YDB mechanism can be described in the following manner:

- upon loading, the stiffer aluminum layers take on most of the load; 
- with continued loading, the aluminum layers begin to yield and load is transferred to the glass layers;

- this load transfer exceeds the strength of the adhesive and is coupled by delamination quickly developing between the adjacent glass and aluminum layers;

- once this delamination reaches a critical length, the aluminum layers buckle;

- since the glass layers themselves remain laterally unsupported, they too will buckle - along with the aluminum layers - providing little contribution to the overall bearing strength of the laminate.

- with increased load, this ultimately precipitates joint collapse and bearing failure. As a consequence of this reasoning, the suggested limiting factor in determining the bearing strength and CCD of a GLARE FML joint is not the overall laminate property; rather, it is governed by the yield strength of the aluminum layers alone. This modified definition of CCD essentially reduces the response of the whole laminate to the response of one of its constituents and one parameter within that constituent: that of the yield strength of the aluminum. Since FMLs are still predominantly aluminum, basing the definition on the major constituent has a physical meaning. As a corollary to the above, it is by virtue of this proposed definition and mechanism that the observed delamination buckling phenomenon is automatically built in, since it implies an intimate relationship between the aluminum yield strength, subsequent delamination and finally buckling of both the aluminum and glass layers. Each of these central tenets of the YDB mechanism will be further investigated both analytically and experimentally in subsequent sections. 


\subsection{FE Modeling for Modified CCD Extraction}

Determination of the modified CCD for the selected and available variants GLARE 22/1-0.4, GLARE 3-5/4-0.4 and GLARE 4-4/3-0.4 was accomplished by generating FE models of suitable geometry and material. Emphasis was placed on enforcing an E/D = 3 to reflect the critical design parameter already identified in the literature. With the models created, failure loads could then be calculated employing Equation 1.3 and the resulting stress distributions could then be plotted.

Investigations into the bearing strength of GLARE conducted by van Rooijen et al. [58] and Buemler [64] found in the literature provided the experimental data required for this initial portion of the present study. Additional analyses including the progressive damage FE modeling as part of the practical methodology relied upon data produced during the experimental investigations of this study to be presented in subsequent sections. A summary of the experimental values found in the literature which were used in this portion of the analysis is shown in Table 2.1.

\subsubsection{Model Geometry and Meshing}

ABAQUS FE [112] software was used for the analysis and the geometry of the models. The fundamental parameter for inducing a bearing failure mode is the E/D ratio which was set equal to three and strictly adhered to throughout the analyses since it represents the critical ratio for fully developing the bearing failure mode. 
Table 2.1: Experimental bearing strength values from Buemler [64] in columns 1, 3 and 4 and van Rooijen et. al. [58] in column 2.

\begin{tabular}{cccccc}
\hline & & GLARE2 & GLARE2 & GLARE3 & GLARE4 \\
\cline { 3 - 6 } Measured Parameter & Unit & MVF = 0.762 & MVF = 0.762 & MVF = 0.643 & MVF = 0.571 \\
\hline $\begin{array}{c}\text { Bearing Yield } \\
\text { Strength }\end{array}$ & $\mathrm{MPa}$ & 717 & 750 & 680 & 646 \\
\hline $\begin{array}{c}\text { Bearing Ultimate } \\
\text { Strength }\end{array}$ & $\mathrm{MPa}$ & 1054 & 1050 & 1018 & 946 \\
\hline
\end{tabular}

Adherence to the $4.8 \mathrm{~mm}$ tested hole diameter was also important to the analysis since an altering of this dimension would produce a different local stress concentration within the tested material. Specimen width was taken to be six times the hole diameter and specimen length was arbitrarily taken to be two times the specimen width. Figure 2.2 shows a typical specimen with a refined mesh for analysis. Dimensions for the individual layers within the laminate and the lay-ups employed for each GLARE variation analyzed in the study are summarized in Table 2.2.

The loading pin and lateral restraint portions of the models were modeled as analytical and discrete rigid surfaces respectively due to their relatively high stiffness when compared to the tested materials.

A half symmetry model was employed in the models for increased computational efficiency and shorter run times. Mesh refinement was applied to provide a coarser mesh in regions of lower stress and sufficiently refined mesh near the highly stressed fastener/hole region. This was accomplished by biasing mesh seeding along edges and 


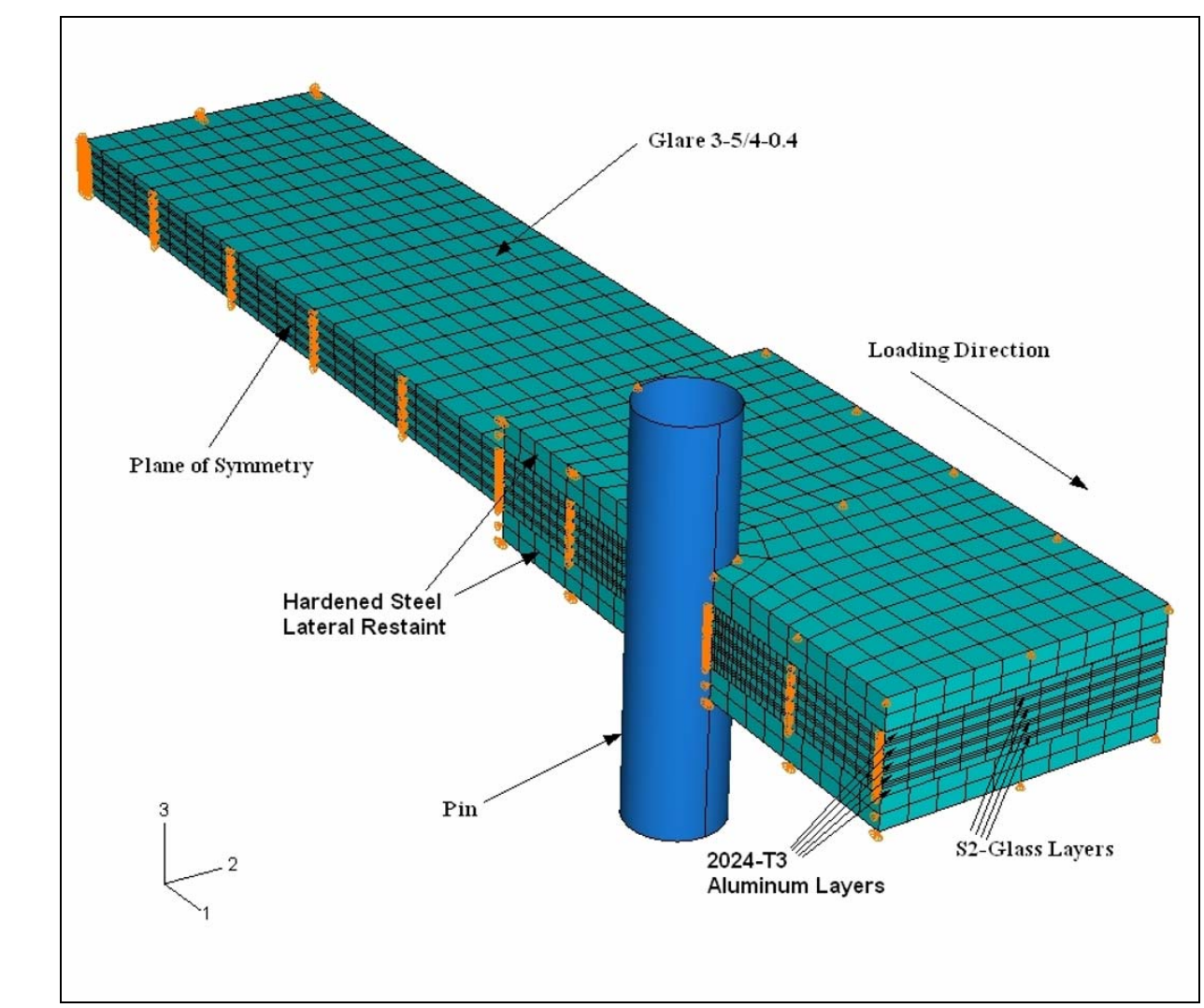

Figure 2.2: Model geometry with a typical mesh and hardened steel lateral restraints for pin bearing analysis.

Table 2.2: A summary of GLARE variants analyzed and their respective dimensions/lay-ups.

\begin{tabular}{ccccc}
\hline GLARE Variant & $\begin{array}{c}\text { Prepreg Lay- } \\
\text { up per Layer }\end{array}$ & $\begin{array}{c}\text { Aluminum Alloy } \\
\text { Thickness [mm] }\end{array}$ & $\begin{array}{c}\text { Prepreg } \\
\text { Thickness per } \\
\text { Orientation } \\
\text { [mm] }\end{array}$ & $\begin{array}{c}\text { Total Laminate } \\
\text { Thickness [mm] }\end{array}$ \\
\hline GLARE2-2/1-0.4 & $0^{\circ}$ & 0.4 & 0.125 & 1.05 \\
\hline GLARE3-5/4-0.4 & $0^{\circ} / 90^{\circ}$ & 0.4 & 0.125 & 3.00 \\
\hline GLARE4-3/2-0.4 & $90^{\circ} / 0^{\circ} / 90^{\circ}$ & 0.4 & 0.125 & 1.95 \\
\hline
\end{tabular}

partitions in a radial fashion towards the hole. A convergence test was conducted between models of increasing mesh refinement in order to verify that mesh convergence had occurred and that the regions of interest had attained sufficient refinement. Each layer of 
the GLARE laminates was modeled as a separate layer of C3D8R elements which are 8 node, linear hexahedral elements with reduced integration and hourglass control [112]. Geometric, surface tie constraints were imposed between the aluminum and glass fibre layers assuming perfect adhesion between adjacent layers. Since the aim of this portion of the study is the extraction of the modified CCD, a linear elastic model with no progressive damage modeling is sufficient. Additional modeling as part of the practical methodology including progressive damage modeling will be presented in Chapter 4 . Interactions between the GLARE laminate and the fastener was modeled with a surfaceto-node (master/slave) contact algorithm for the detection and calculation of normal contact forces. In addition to normal forces, friction was also modeled between the fastener and laminate using a coefficient of friction equal 0.1 . Interaction between the lateral restraint and the laminate was also modeled for contact, but no friction model was applied.

\subsubsection{Boundary Conditions and Load Case}

Boundary conditions were imposed for all degrees of freedom on the far end of the models to simulate clamping. Displacement in the $\mathrm{U}_{2}$ direction along the 1-3 mid-plane of the models was restricted to simulate symmetry and the lateral restraints were fixed in their $\mathrm{U}_{3}$ displacements to simulate a finger tightened bolt. A gap of $0.01 \mathrm{~mm}$ in the $\mathrm{U}_{3}$ direction was also left between the lateral restraint and the tested material since finger tightening without any clamping pressure is never truly a tight fit. Failure loads were applied along the 1 (longitudinal) axis, to a centrally located reference point of the 
analytical rigid fastener in the models. A summary of the calculated failure loads as they were applied to the half symmetry models is located in Table 2.3 .

Table 2.3: A summary of the calculated failure loads and loads applied in the symmetry models.

\begin{tabular}{ccc}
\hline GLARE Variant & Calculated Failure Load [N] & $\begin{array}{c}\text { Failure Load Applied in } \\
\text { Symmetry Model [N] }\end{array}$ \\
\hline GLARE2-2/1-0.4 & 5292 & 2646 \\
\hline GLARE3-5/4-0.4 & 14659 & 7239.5 \\
\hline GLARE4-3/2-0.4 & 8855 & 4427.5 \\
\hline
\end{tabular}

\subsubsection{Material Properties}

The material properties of both the 2024-T3 aluminum layers and the $\mathrm{S}_{2}$-glass fibre prepreg layers are given in Table 2.4. Material properties were submitted to ABAQUS via the ELASTIC $\rightarrow$ ENGINEERING CONSTANTS feature available which is used to define orthotropic materials through their nine engineering constants. Failure criteria were not implemented into the model as well as the residual stresses that occur during the manufacturing process for GLARE. Consideration of these factors is beyond the scope of this portion of the study but receive proper consideration in Chapter 4 .

Table 2.4: Material properties for aluminum 2024-T3 and $S_{2}$-glass fibre.

\begin{tabular}{cccccccccc}
\hline Material & $\begin{array}{c}E_{11} \\
{[G P a]}\end{array}$ & $\begin{array}{c}E_{22} \\
{[G P a]}\end{array}$ & $\begin{array}{c}E_{33} \\
{[G P A]}\end{array}$ & $\boldsymbol{v}_{12}$ & $\boldsymbol{v}_{13}$ & $\boldsymbol{v}_{23}$ & $\begin{array}{c}\boldsymbol{G}_{12} \\
{[G P a]}\end{array}$ & $\begin{array}{c}\boldsymbol{G}_{13} \\
{[G P a]}\end{array}$ & $\begin{array}{c}\boldsymbol{G}_{23} \\
{[\mathrm{GPPa}]}\end{array}$ \\
\hline 2024-T3 & 72.4 & 72.4 & 72.4 & 0.33 & 0.33 & 0.33 & 27.6 & 27.6 & 27.6 \\
\hline $\boldsymbol{S}_{\mathbf{2}}$-Glass & 53.98 & 9.412 & 9.412 & 0.33 & 0.33 & 0.33 & 2.7 & 2.7 & 1.6875 \\
\hline
\end{tabular}




\subsection{FE Analysis Results}

Figure 2.3 and Figure 2.4 show some typical results of the FE analysis for the GLARE22/1-0.4 and GLARE3-5/4-0.4 pin bearing models. The significance of the figure(s) is the location of the highest stresses in the model. The elements directly in contact with the fastener experience highly localized stress, which decays rapidly moving away from the fastener hole in the loading direction.

In addition, a close inspection of the deformed shape with the glass layers removed for clarity as depicted in Figure 2.5 shows the buckling of the aluminum layers as observed in the literature. Buckling is most pronounced in the outermost layers of aluminum and is symmetrical about the middle layer of aluminum.

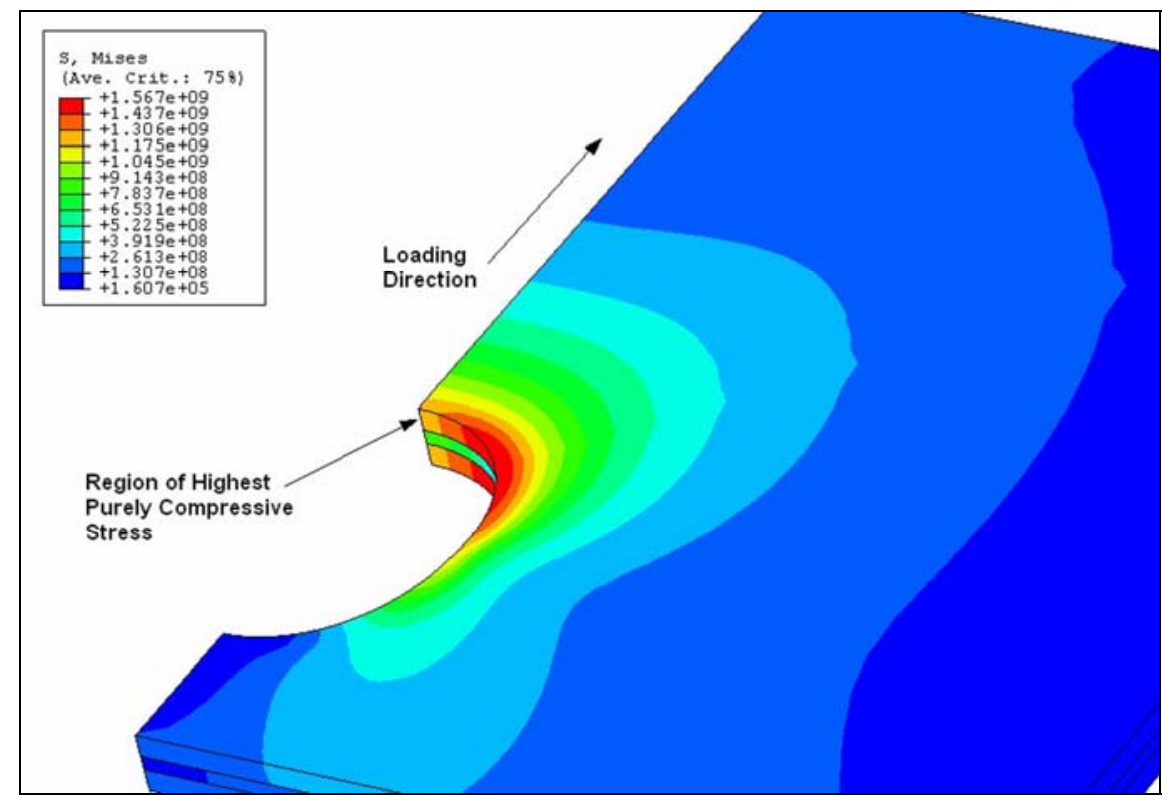

Figure 2.3: Typical Von Mises stress distribution for GLARE 2-2/1-0.4 showing region of highest compressive stress. 


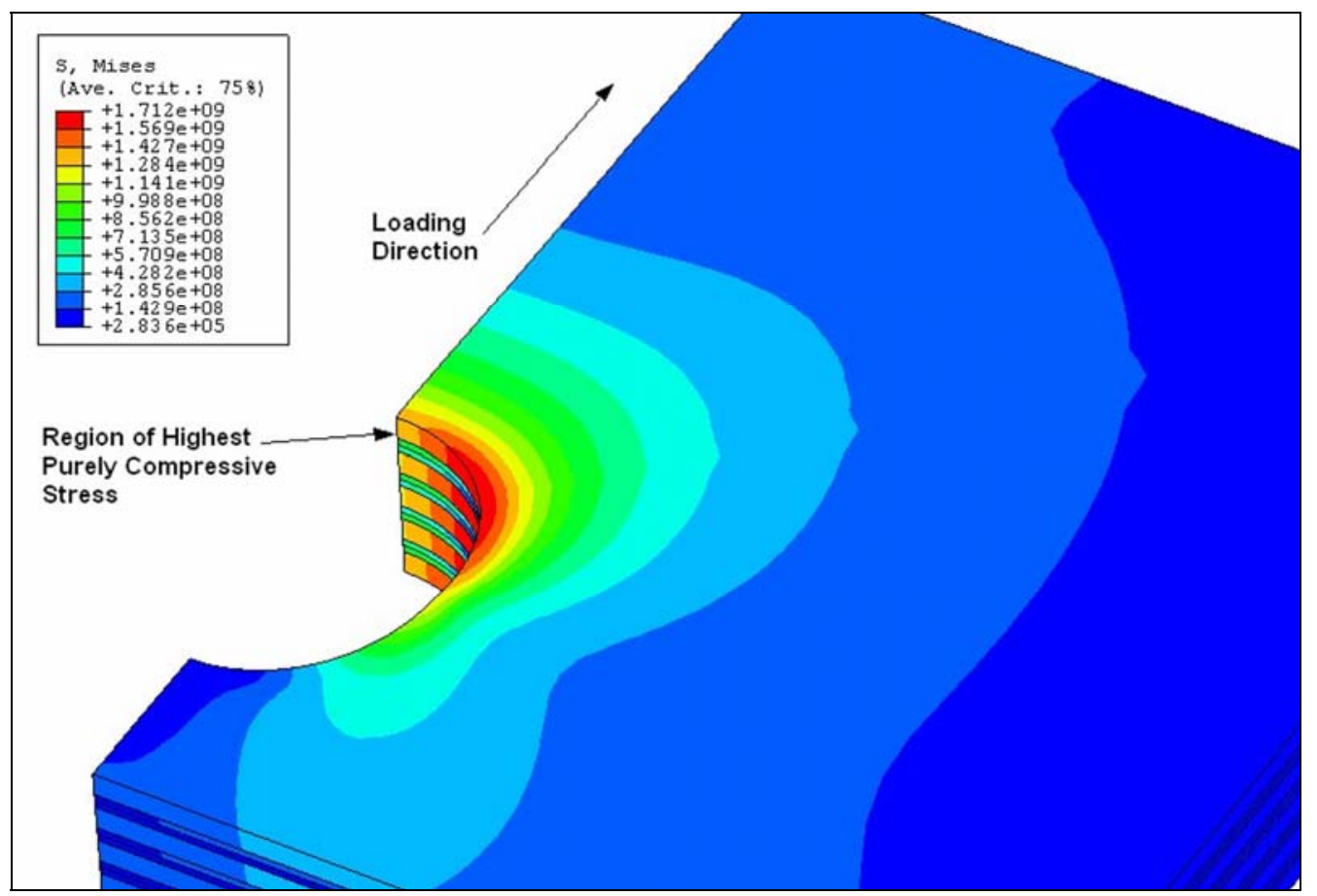

Figure 2.4: Typical Von Mises stress distribution for GLARE3-5/4-0.4 showing region of highest compressive stress.

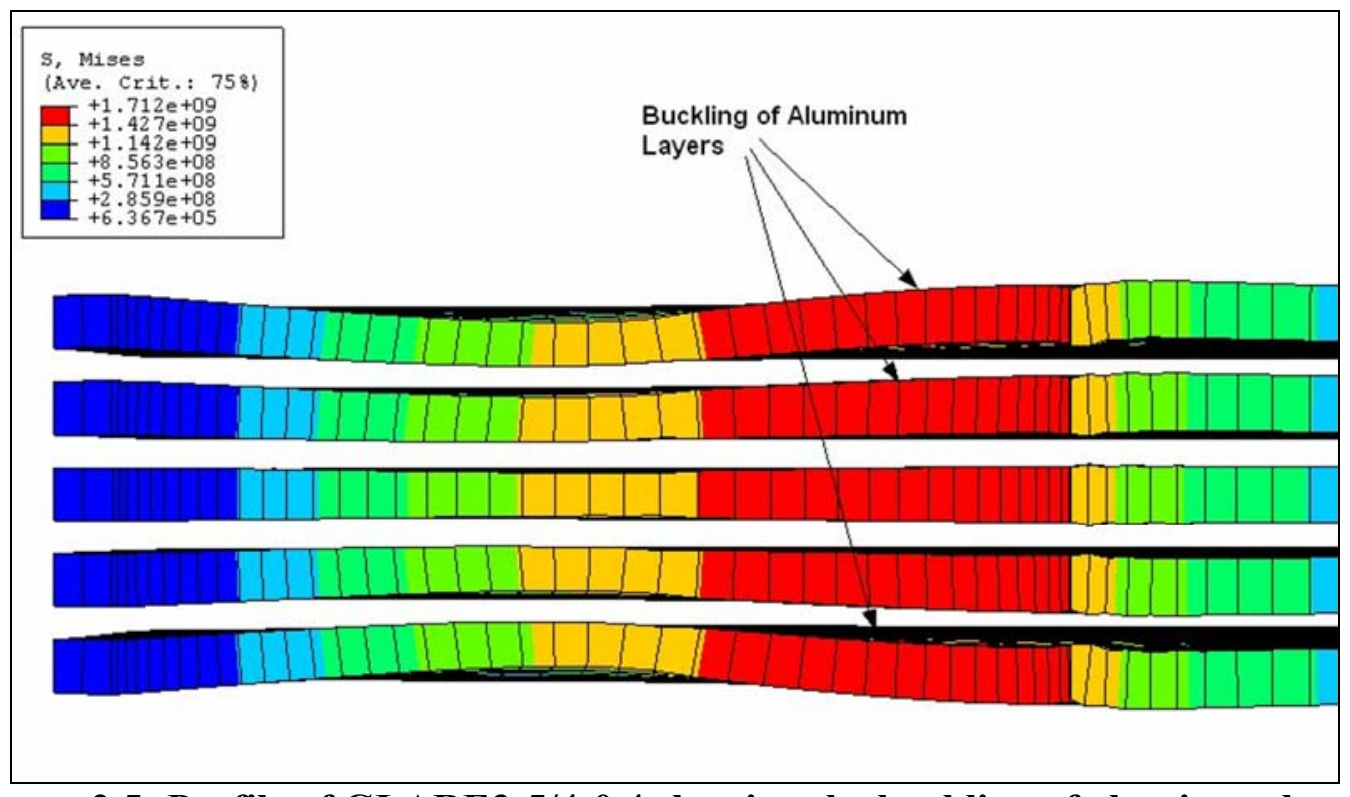

Figure 2.5: Profile of GLARE3-5/4-0.4 showing the buckling of aluminum layers with a scale factor of 25 for clarity. 


\subsubsection{Plot Generation via User Defined Nodal Paths}

A plot of compressive stress versus distance from the fastener hole can be generated by first plotting a nodal path of interest on the deformed model. Figure 2.6 shows a typical node path plotted by the user which radiates directly outward from the fastener hole until it reaches the end of the specimen. Nodal values of any requested field output (Von Mises, $\mathrm{S}_{11}$ stress, Tresca, nodal displacement, etc.) can be retrieved from each node along the path. The plotting of nodal paths is applied to each of the constituent layers and subsequent $\mathrm{x}-\mathrm{y}$ data plots of the stress distribution for specific layers can be readily generated.

The analysis results in Figure 2.7 and Figure 2.8 show the stress distribution of the combined Von Mises stress in the compressive region of both the aluminum and glass layers of all the GLARE variants analyzed. Although several field variables were collected, plotted and CCD calculations were performed, it was the combined stresses of Von Mises and Tresca whose values are most relevant. More specifically, since it is well known that the Tresca stress is more conservative than the Von Mises stress, emphasis was placed on the latter. In a pin bearing case, the induced stress state is complex giving rise to stress components in more than one direction. Therefore the stress components along the 2 (transverse) and 3 (thickness) directions of the model, although smaller, cannot be omitted since they will contribute to the overall compressive stress field and will affect the magnitude of the modified CCD. 


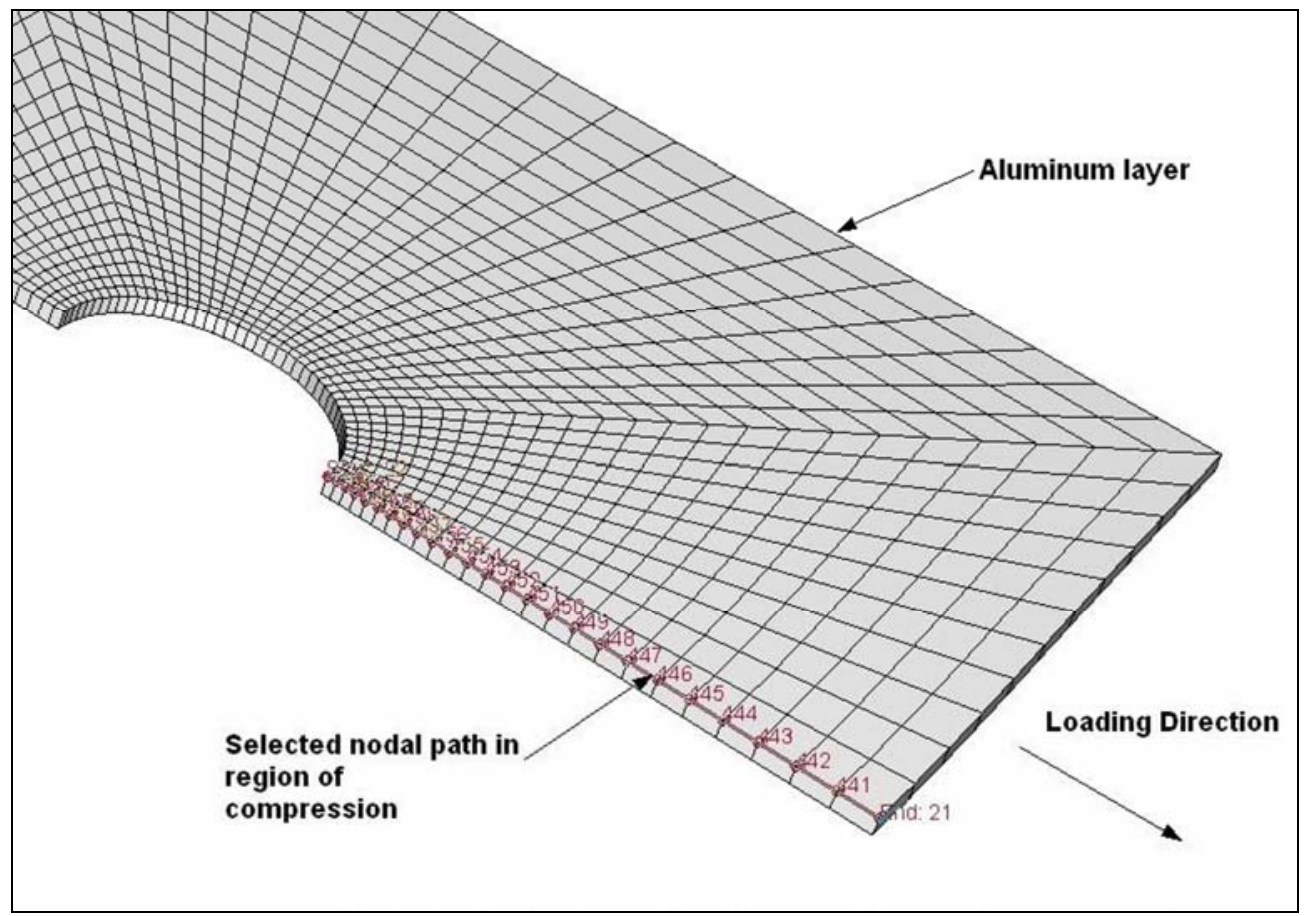

Figure 2.6: User defined nodal path parallel to the 1 (longitudinal) axis in the aluminum layer of a typical pin bearing model.

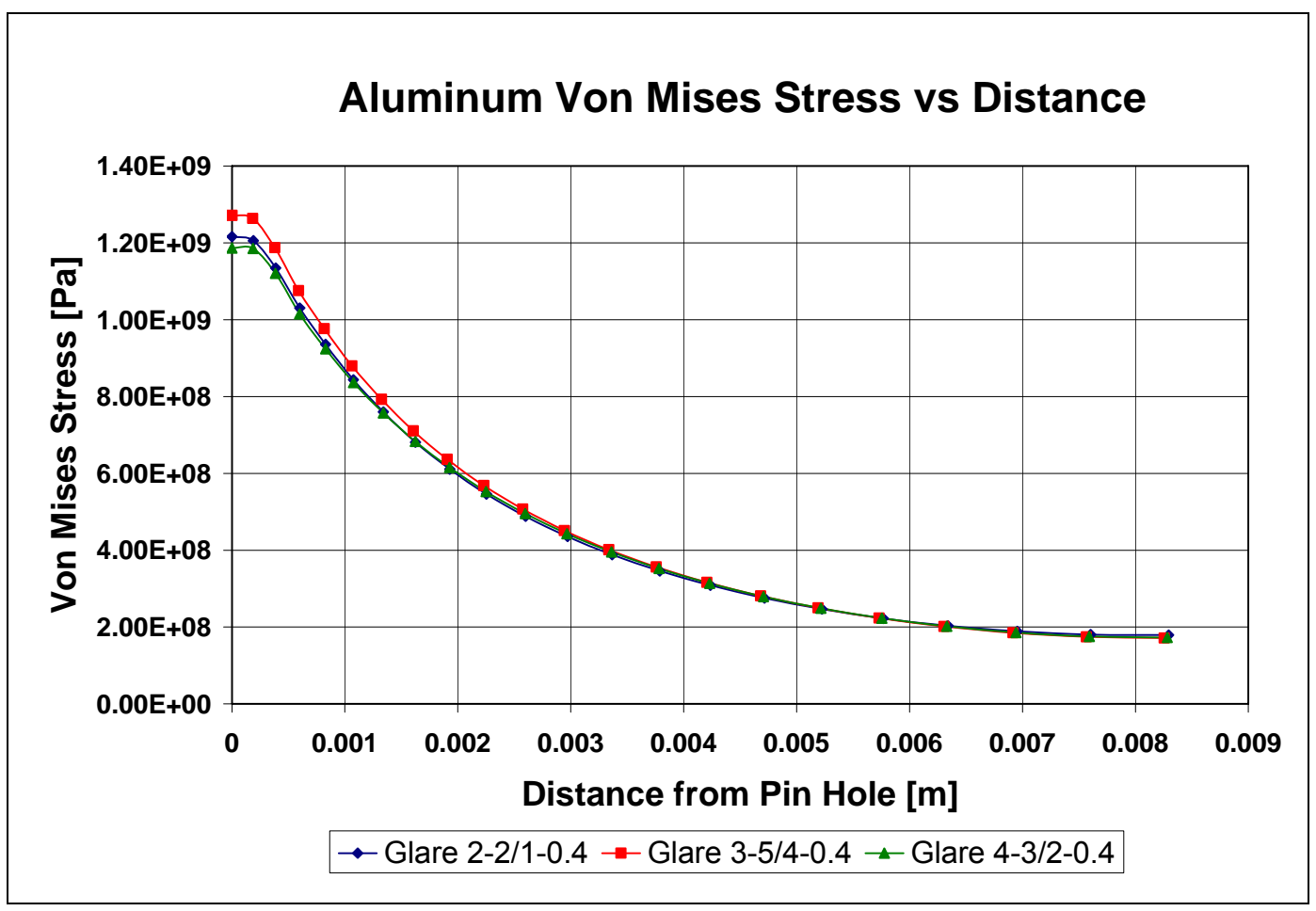

Figure 2.7: Comparison of the Von Mises stress distribution in the aluminum layers for all GLARE variants analyzed. 


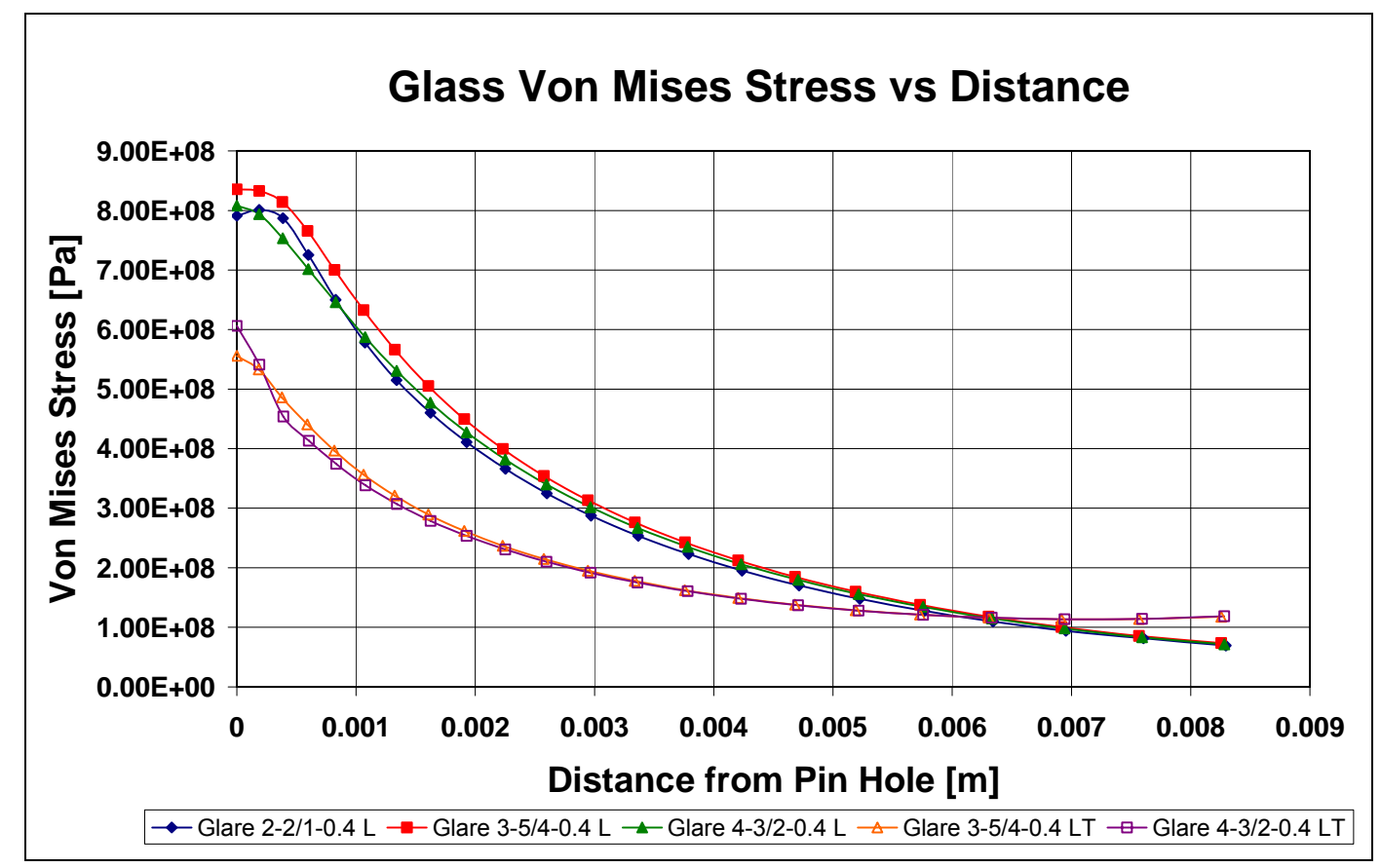

Figure 2.8: Comparison of the Von Mises stress distribution in the glass layers in both the longitudinal and transverse directions for all GLARE variants analyzed.

It should be mentioned that the maximum stress values from Figure 2.7 and Figure 2.8 seem to be excessively high and much more so than the ultimate tensile strength of the laminate. This comes from the very essence of the charactetistic dimension method. In short, the characteristic dimension method allows for the use of a linear elastic analysis without having to include complex, non-linear effects associated with high loads. It is understood that due to the nature of the linear elastic analysis, the stresses generated at the hole edge are raised by a stress concentration factor to create a stress value much higher than the yield or ultimate strength. It is this fact that allows the analyst to not focus on this inner region and to conduct the failure analysis at the characteristic curve instead. 


\subsection{Calculation of a Modified CCD}

If we examine the drop in stress moving away from the fastener hole we see that the values of stress approaches the yield and ultimate strengths of the 2024-T3 aluminum. Of paramount importance though is the extraction of modified CCD values with sufficient accuracy. This was accomplished by specifying the precise value of the yield strength of the aluminum $\left(\mathrm{y}=\sigma_{\mathrm{y}, \mathrm{Al}}=345 \mathrm{MPa}\right)$. Upper and lower bounds for the point of interest (ie. the corresponding value on the $\mathrm{x}$ axis defining the distance) were then interpolated with a third order polynomial function at the specified yield value. This convention was followed for all the calculations and values for $\mathrm{CCD}$ as extracted from the analysed plots corresponding to the longitudinal stress (1-axis) and for two combined stress criteria. The results are summarized in Table 2.5 .

Table 2.5: Calculated modified CCD for one stress component and two combined stresses for all GLARE variants analyzed.

\begin{tabular}{ccccc}
\hline GLARE Variant & Constituent & $\begin{array}{c}\text { CCD for S11 } \\
\text { [mm] }\end{array}$ & $\begin{array}{c}\text { CCD for Von } \\
\text { Mises [mm] }\end{array}$ & $\begin{array}{c}\text { CCD for Tresca } \\
\text { [mm] }\end{array}$ \\
\hline \multirow{2}{*}{ GLARE2-2/1-0.4 } & $\mathrm{Al}$ 2024-T3 & 2.814 & 3.800 & 4.350 \\
\cline { 2 - 5 } & $\mathrm{S}_{2}$-Glass L & 2.328 & 2.431 & 2.514 \\
\hline \multirow{3}{*}{ GLARE3-5/4-0.4 } & $\mathrm{Al}$ 2024-T3 & 3.157 & 3.886 & 4.362 \\
\cline { 2 - 5 } & $\mathrm{S}_{2}$-Glass L & 2.571 & 2.659 & 2.721 \\
\cline { 2 - 5 } & $\mathrm{S}_{2}$-Glass LT & 0.719 & 1.147 & 1.404 \\
\hline \multirow{3}{*}{ GLARE4-3/2-0.4 } & $\mathrm{Al}_{\text {2024-T3 }}$ & 3.032 & 3.866 & 4.372 \\
\cline { 2 - 5 } & $\mathrm{S}_{2}$-Glass L & 2.471 & 2.555 & 2.619 \\
\cline { 2 - 5 } & $\mathrm{S}_{2}$-Glass LT & 0.628 & 1.034 & 1.303 \\
\hline
\end{tabular}

When examining the values for modified CCD given in Table 2.5, we see different values of CCD for the aluminum and for the glass fibre. This is due to the differences in stiffness associated with each material. 
Since FMLs are mostly aluminum and since it is proposed that the yielding of the aluminum is what leads to load transfer, delamination and finally buckling as per the YDB mechanism, the modified CCD for the aluminum becomes the parameter of interest for the purposes of the present investigation.

It is because there is such a large difference in material properties between the aluminum layers and the glass layers that a conventional definition of $\mathrm{CCD}$ is not applicable to GLARE without modification. This is backed by an understanding in the literature that the aluminum plays a significant role in a bearing failure mode for GLARE joints. 


\subsection{Orthotropic Plate Buckling Analysis of the Laterally Unsupported Glass Prepreg Plies}

In order to investigate the observed delamination buckling phenomenon and examine if the contribution of the glass prepreg plies to overall bearing strength is small, an orthotropic plate buckling analysis of the laterally unsupported regions of the glass layers was conducted.

The dimensions of the delaminated/laterally unsupported regions of prepreg as part of the YDB mechanism was based on the conservative assumption that delamination has, at the very least, occurred in the area under compressive stress next to the fastener hole where the stress profile in the aluminum layers (as plotted against increasing distance from the fastener hole) has exceeded the yield strength of aluminum. This is based on the idea that it is the yielding of aluminum which leads to the transfer of load to the glass through the adhesive which leads to delamination.

Therefore, if we examine the stress profile in the aluminum layers, in both the $x$ (transverse) and y (longitudinal) directions moving away from the fastener hole, we can estimate that the glass layers directly adjacent and previously bonded to the yielded aluminum layers have delaminated and are laterally unsupported over an $\mathrm{x}$ and $\mathrm{y}$ distance until the yield strength of aluminum is reached in the aluminum layer stress profile related to each direction. This is a direct consequence of the proposed YDB mechanism and based of the yield strength of aluminum as a governing parameter in joint collapse. In the analysis, the delaminated area was accomplished by generating user defined nodal paths within the model along $\mathrm{x}$ and $\mathrm{y}$ and evaluating the output for the selected field 
variables $\left(\mathrm{S}_{11}\right.$ stress, Von Mises, Tresca) at each nodal point along the path until their outputted values equalled the yield strength of aluminum. This provided a well defined distance to the desired boundaries of the delaminated/laterally unsupported region. The result is a conservative, rectangular shape for the adjacent, delaminated/laterally unsupported region in the glass layers with its boundaries defined as the distance from the fastener hole at which the stress profile in the aluminum layers, for each direction, has decreased to the yield strength of the aluminum as shown in Figure 2.9. Since each stress criterion calculated yields a different stress distribution about the fastener hole, the dimensions of the laterally unsupported regions will vary and thus produce different critical buckling loads.

In reality, the delaminated region may be slightly larger than the aforementioned yielded area and not necessarily rectangular in shape. However, defining it in this manner allows

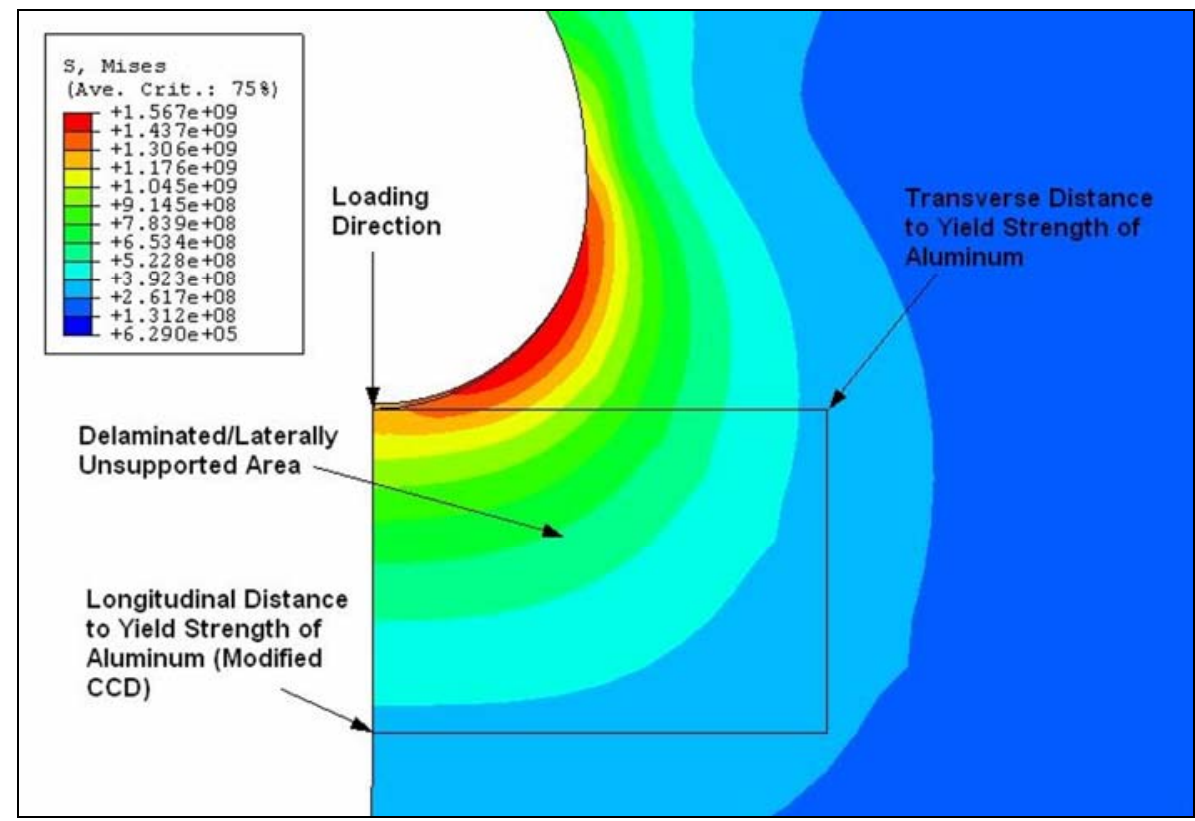

Figure 2.9: Schematic of the delaminated/laterally unsupported area used in the orthotropic plate buckling analysis based on the results of the FE analysis. 
for the generation of precise boundaries based on the results of the FE analysis in a minimal sense. A larger, laterally unsupported area would only buckle sooner at lower applied loads. The objective of the orthotropic plate buckling analysis is to determine whether or not the glass layers will buckle at less than or equal to the yield stress of the aluminum. If a smaller, laterally unsupported area buckles than a larger area will invariably buckle as well. The defined boundaries for the laterally unsupported regions of the glass prepreg layers for each stress criterion calculated are presented in Table 2.6. The thickness of the rectangular glass prepreg regions varies as each grade of GLARE does with $0.250 \mathrm{~mm}, 0.250 \mathrm{~mm}$ and $0.375 \mathrm{~mm}$ applying to GLARE2, GLARE3 and GLARE4 respectively. This is the result of the number of individual prepreg layers within each fibre layer of the laminate.

These delaminated regions, with their accompanying thicknesses, define orthotropic plates subjected to a buckling load configuration. Using classical lamination theory and

Table 2.6: Dimensions of the delaminated/laterally unsupported regions of the glass prepreg layers used in the orthotropic plate buckling analysis.

\begin{tabular}{ccccccc}
\hline & \multicolumn{2}{c}{$\boldsymbol{S}_{11}$} & \multicolumn{2}{c}{ Von Mises } & \multicolumn{2}{c}{ Tresca } \\
\cline { 2 - 7 } GLARE Variant & $\begin{array}{c}\text { Length } \\
\text { [mm] }\end{array}$ & $\begin{array}{c}\text { Width } \\
\text { [mm] }\end{array}$ & $\begin{array}{c}\text { Length } \\
{[\mathbf{m m}]}\end{array}$ & $\begin{array}{c}\text { Width } \\
{[\mathbf{m m}]}\end{array}$ & $\begin{array}{c}\text { Length } \\
{[\mathbf{m m}]}\end{array}$ & $\begin{array}{c}\text { Width } \\
\text { [mm] }\end{array}$ \\
\hline GLARE2-2/1-0.4 & 2.814 & 1.974 & 3.800 & 5.269 & 4.350 & 5.774 \\
\hline GLARE3-5/4-0.4 & 3.150 & 2.039 & 3.886 & 5.577 & 4.362 & 6.078 \\
\hline GLARE4-3/2-0.4 & 3.032 & 2.014 & 3.866 & 5.438 & 4.372 & 5.928 \\
\hline
\end{tabular}


the aforementioned material properties, the A, B and D matrices for the orthotropic plates of glass prepreg was then calculated.

The critical buckling load per unit length for an orthotropic plate subject to various boundary conditions can be found using Equation (1.4) [113]:

$$
P_{c r}=k \frac{\pi}{b^{2}} \sqrt{\left(D_{11} D_{22}\right)}
$$

where $D_{11}$ and $D_{22}$ are entries in the bending stiffness matrix specific to each lay-up and $k$ is the buckling constant dependant on the prescribed boundary conditions. The value of $k$ is a function of two dimensionless parameters $\alpha$ and $\beta$ for the two edge constraints using Equation (2.2) and (2.3) [113]:

$$
\begin{aligned}
& \alpha=\frac{D_{12}+2 D_{66}}{\sqrt{\left(D_{11} D_{22}\right)}} \\
& \beta=\left(\frac{a}{b}\right) \sqrt{\frac{D_{22}}{D_{11}}}
\end{aligned}
$$

where $a$ is the specimen length and $b$ is the specimen width. Plots of $\alpha$ and $\beta$ are generated for various boundary conditions and values of $k$ can be found as a function of them.

Once the critical buckling load per unit length is calculated, a value of critical buckling load followed by critical normal stress applied to the loaded edge of the plate can be calculated based on the dimensions of the plate, again, dependant on the stress criterion in question.

Two sets of boundary conditions were examined for the orthotropic plate buckling analysis conducted herein. The first case enforced fully clamped boundary conditions on 
the three unloaded edges of the plate and the remaining loaded edge was simply supported. The second load case enforced fully clamped boundary conditions on all sides of the plate. If the critical buckling stress for the examined load cases is less than or equal to the yield strength of the aluminum layers it can be said that the glass prepreg layers do not increase the bearing strength of the laminate, in a bearing failure mode, and the yielding of the aluminum layers, coupled with the observed delamination buckling is the main mechanism of joint failure - manifestly, the YDB mechanism.

\subsubsection{Results of the Orthotropic Plate Buckling Analysis}

The results of the orthotropic plate buckling analysis for both the clamped-clampedclamped-simply supported and the fully clamped loading configurations are shown in Table 2.7 and Table 2.8 respectively.

If we examine these results it is evident that the stress required to buckle the laterally unsupported orthotropic plates as a result of the delamination, for all of the examined stress criteria and both boundary condition configurations, is much less than the yield stress of the aluminum layers. Therefore, it can be said that these results indicate that the contribution of the glass prepreg layers to the bearing strength of the laminate in a bearing failure mode is small and can be deemed negligible. This supports the hypothesis of the YDB mechanism and is an entirely intuitive result. 
Table 2.7: Critical buckling constants, loads and normal stresses for the clampedclamped-clamped-simply supported loading configuration.

\begin{tabular}{|c|c|c|c|c|c|c|c|c|c|}
\hline \multirow[b]{2}{*}{ GLARE Variant } & \multicolumn{3}{|c|}{ s11 } & \multicolumn{3}{|c|}{ Von Mises } & \multicolumn{3}{|c|}{ Tresca } \\
\hline & $k$ & $\boldsymbol{P}_{c r}(\mathbf{N})$ & $\begin{array}{c}\sigma_{c r} \\
(\mathrm{MPa})\end{array}$ & $k$ & $\begin{array}{l}P_{c r} \\
(N)\end{array}$ & $\begin{array}{c}\sigma_{c r} \\
(\mathrm{MPa})\end{array}$ & $k$ & $\begin{array}{l}P_{c r} \\
(N)\end{array}$ & $\begin{array}{c}\sigma_{c r} \\
(M P a)\end{array}$ \\
\hline GLARE2-2/1-0.4 & 5.500 & 132.66 & 11.94 & 5.750 & 19.47 & 0.486 & 5.750 & 16.21 & 0.323 \\
\hline GLARE3-5/4-0.4 & 5.313 & 42.22 & 3.39 & 5.188 & 5.51 & 0.127 & 5.188 & 4.64 & 0.088 \\
\hline GLARE4-3/2-0.4 & 5.375 & 168.26 & 9.18 & 5.750 & 24.69 & 0.391 & 5.625 & 20.32 & 0.261 \\
\hline
\end{tabular}

Table 2.8: Critical buckling constants, loads and normal stresses for the fully clamped loading configuration.

\begin{tabular}{|c|c|c|c|c|c|c|c|c|c|}
\hline \multirow{2}{*}{ GLARE Variant } & \multicolumn{3}{|c|}{ s11 } & \multicolumn{3}{|c|}{ Von Mises } & \multicolumn{3}{|c|}{ Tresca } \\
\hline & $k$ & $P_{c r}(N)$ & $\begin{array}{c}\sigma_{c r} \\
(\mathrm{MPa})\end{array}$ & $k$ & $P_{c r}(N)$ & $\begin{array}{c}\sigma_{c r} \\
(\mathrm{MPa})\end{array}$ & $k$ & $\begin{array}{l}P_{c r} \\
(N)\end{array}$ & $\begin{array}{c}\sigma_{c r} \\
(M P a)\end{array}$ \\
\hline GLARE2-2/1-0.4 & 14 & 337.68 & 30.39 & 15 & 50.78 & 1.27 & 15.25 & 42.99 & 0.856 \\
\hline GLARE3-5/4-0.4 & 6.5 & 51.66 & 4.15 & 11 & 11.69 & 0.269 & 10.5 & 9.39 & 0.177 \\
\hline GLARE4-3/2-0.4 & 11 & 344.35 & 18.79 & 15 & 64.41 & 1.02 & 14.75 & 53.29 & 0.685 \\
\hline
\end{tabular}

\subsection{Chapter Summary}

In this chapter, a modified definition of the CCD was presented and applied to GLARE

FMLs. The definition arises from the idea that in pin bearing, the yield strength of the aluminum determines the onset of failure through delamination and buckling. The lead to the proposal of the YDB mechanism for pin bearing at the critical $\mathrm{E} / \mathrm{D}=3$ ratio, which is valuable to design. Values of modified CCD were calculated for several GLARE 
variants. Buckling analysis of the glass layers suggest that they provide a small to negligible contribution to the overall bearing strength of FMLs in pin bearing once delamination has occurred. Experimental investigations to examine the modified CCD and YDB mechanism will be presented next. 


\section{Chapter Three: Bearing Strength and Tensile Tests on Quasi-Isotropic and Orthotropic FMLs}

The aim of this portion of the study is to develop and implement, based upon and extending from current testing standards, an experimental methodology designed to investigate the measurement of the strain field generated as a result of pin bearing. The detection of yield in the aluminum layers is an important portion of this study to experimentally investigate the modified CCD and YDB mechanism as part of the practical progressive damage methodology.

\subsection{Pin Bearing Experiments}

The developed test fixtures, fabricated specimens and experimental protocol enlisted in this study are discussed in the next few sections. Emphasis was placed on capturing the behaviour of the laminate throughout the entire test window from more than one point of view to detect both yielding and buckling of the aluminum layers. 
Previous investigations into the bearing strength of FMLs have, in general, been based on two ASTM standards, viz., ASTM D953 [68] and ASTM E238 [69]. The former of these two is the standard test method for the bearing strength of plastics (both isotropic and anisotropic) and represents the basis of the extended experimental protocol.

The decision to employ a pin bearing loading configuration rather than a bolt bearing one rests on the characteristic absence of lateral restraints. It has been mentioned previously in this thesis that laminated structures exhibit significant increases in bearing strength in the presence of lateral restraints and that the pin bearing case is the most conservative scenario. The principal cause of this observed increase is attributed to the restriction of delamination as a result of the added boundary condition. Since one of the principal aims of this portion of the study was to examine the local stress-strain behaviour and yielding as a result of a pin bearing load and delamination buckling, failure in this manner is only to be encouraged via a lack of lateral restriction.

\subsubsection{Pin Bearing Test Fixtures}

Figure 3.1 and Figure 3.2 depict the test fixtures designed and manufactured for both the quasi-isotropic and orthotropic specimens. This represents the first extension of ASTM D953 [68]. In a standard, tension based, pin bearing loading fixture, the hardened side plates are machined with two holes in their upper portion(s) for bolting the fixture together and a single, $6.35 \mathrm{~mm}$ hole in the lower portion for insertion of the loading pin. The loading fixtures manufactured for this investigation were machined with 


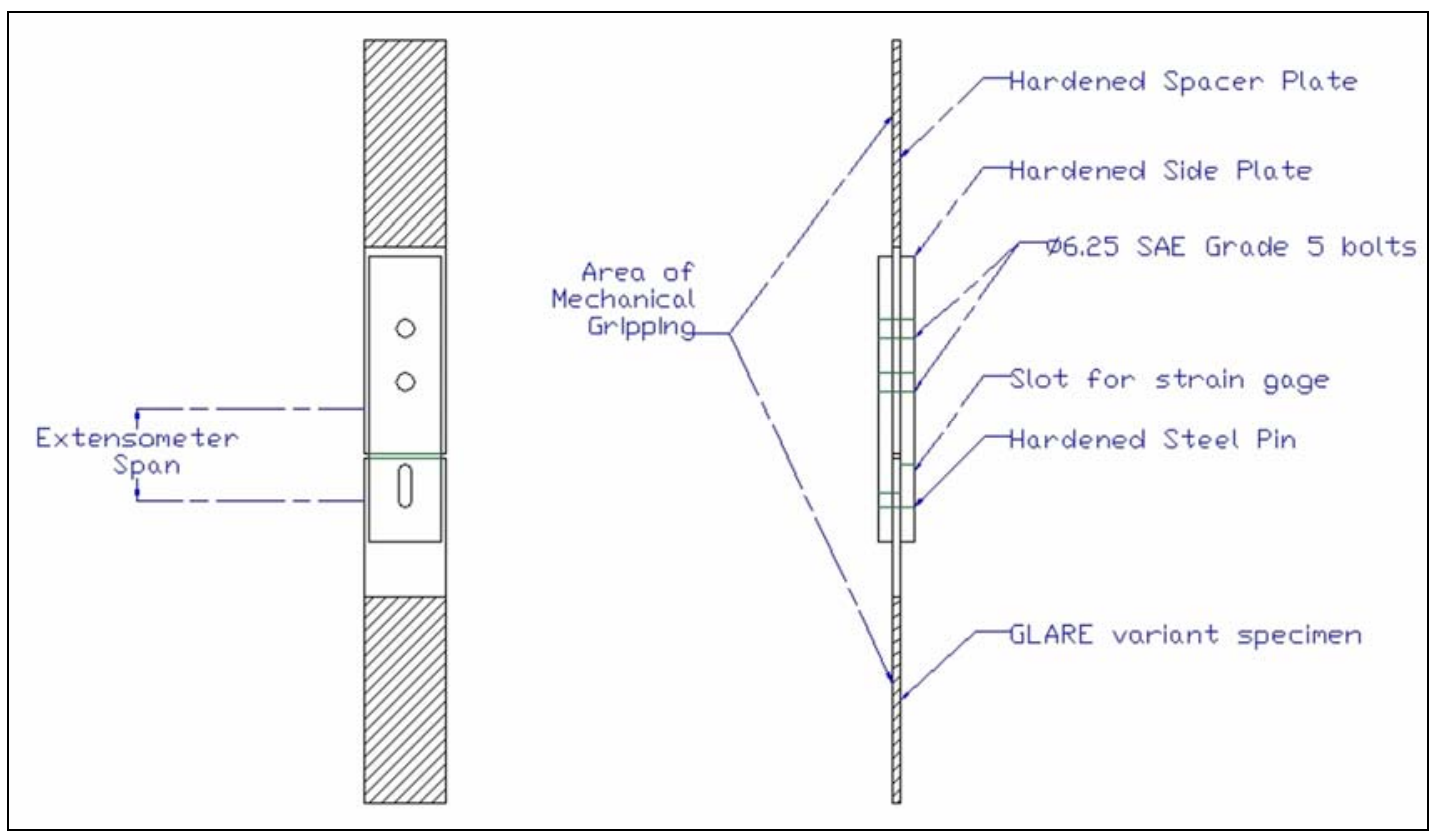

Figure 3.1: Tension based loading fixture with machined slot for the facilitation of strain gage application on the quasi-isotropic specimens.

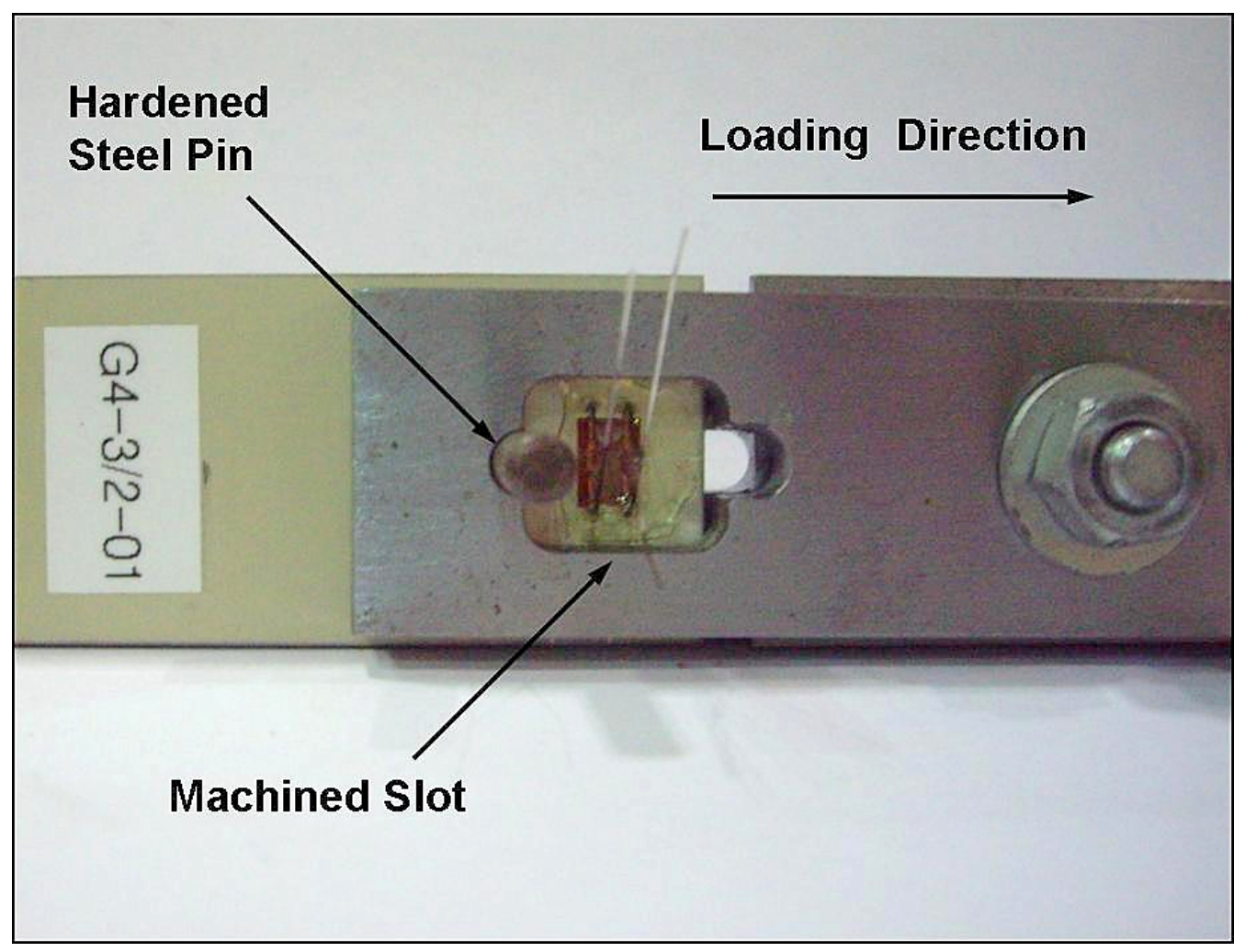

Figure 3.2: Hardened steel side plate with expanded slot as part of the tension based loading fixture for the orthotropic specimens. 
a $4.8 \mathrm{~mm}$ diameter loading pin hole for easier application to practical joint design and the assurance of geometric similarity to the FE study discussed earlier and progressive damage FE study to be discussed in subsequent sections. In addition, one of the hardened steel side plates also included a precision machined slot with semi-circular ends of equal radius to the loading pin hole. This slot was included in the design in order to facilitate the application of a bonded strain gage onto the specimen in question. This bonded strain gage enables the measurement of strain from a local scheme in addition to a global one. Details pertaining to the motivation, selection and application of the bonded strain gage will be discussed in the next section.

Once assembled, the hardened steel loading fixture has a much higher relative stiffness, ensuring that any displacement in the system is attributed solely to the deformation of the specimen material surrounding the loading pin/hole.

\subsubsection{Fabrication of Tested Samples}

The bearing specimens were fabricated from sheets of GLARE3-5/4-0.3, GLARE3-4/30.3 and GLARE4-3/2-0.3, employing abrasive water jet cutting to minimize delamination of the constituent layers. For the GLARE3 specimens, there are an equal number of unidirectional prepreg plies in both the longitudinal direction (rolling direction of aluminum) and transverse directions in the case of GLARE3-5/4-0.3 and an approximately equal number in the case of GLARE3-4/3-0.3, therefore, they can be considered quasi-isotropic. A GLARE 4 variant implies that there are three, unidirectional, $0.125 \mathrm{~mm}$ glass prepreg plies per composite layer: one oriented in the 
longitudinal direction (rolling direction of aluminum) and two oriented transversely so making it orthotropic in nature.

All specimens were fabricated to be $120.6 \mathrm{~mm}$ in length and $28.8 \mathrm{~mm}$ in width. Figure 3.3 shows a typical pin bearing test specimen with its major dimensions shown and Table 3.1 summarizes the GLARE variants tested.

The $4.8 \mathrm{~mm}$ diameter loading pin hole was reamed to correct size to ensure sharp and smooth, but unpolished edges. The centre of the hole was positioned $14.4 \mathrm{~mm}$ away from the specimen edge, thus imposing an $\mathrm{E} / \mathrm{D}=3$ and representing the critical design ratio. The previously mentioned specimen width of $28.8 \mathrm{~mm}$ was designed to enforce a W/D = 6. The motivation behind the selection of these values was to examine the critical design case for the bearing failure mode as previously discussed.

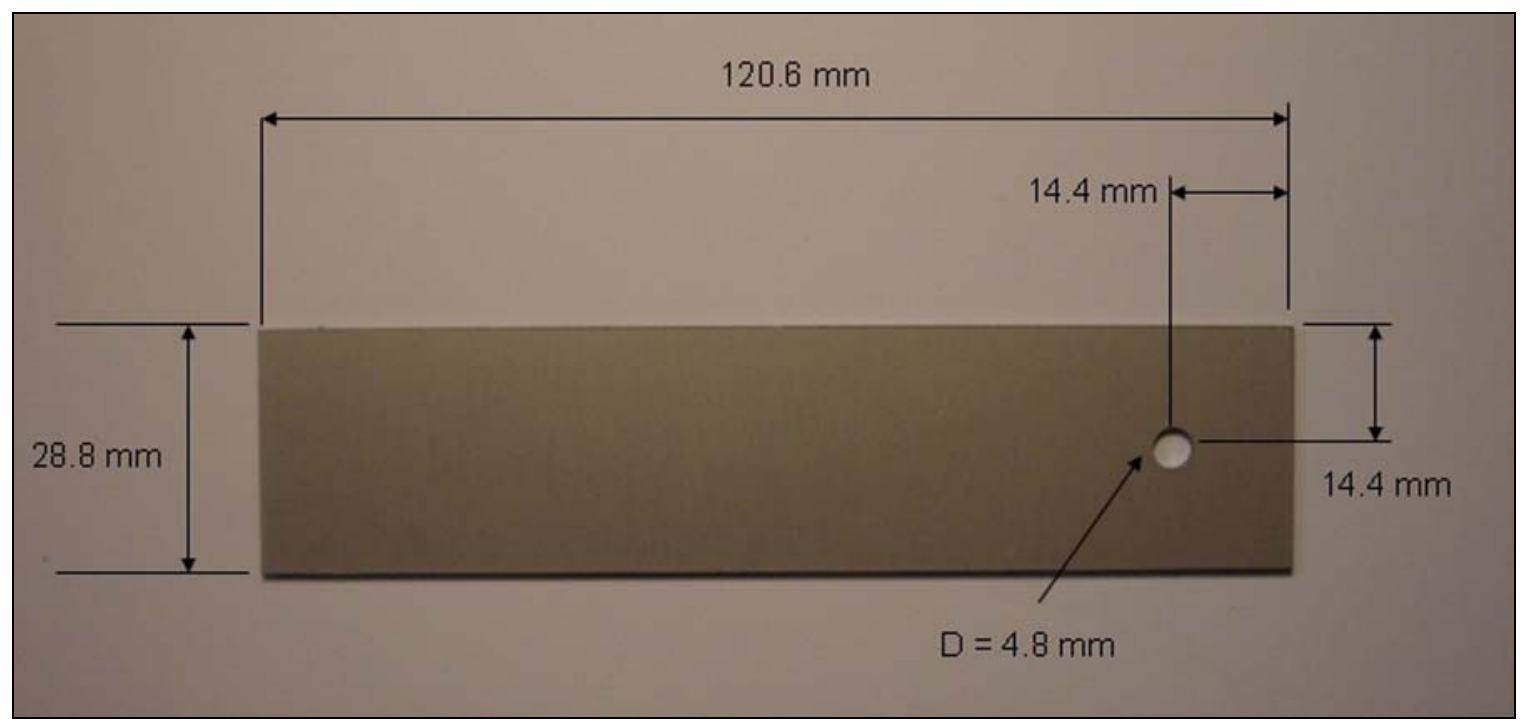

Figure 3.3: Typical pin bearing GLARE specimen with its major dimensions shown. 
Table 3.1: A summary of GLARE variants tested and their respective dimension/lay-ups.

\begin{tabular}{lcccc}
\hline GLARE Variant & $\begin{array}{c}\text { Prepreg Lay-up } \\
\text { per Layer }\end{array}$ & $\begin{array}{c}\text { Aluminum Alloy } \\
\text { Thickness [mm] }\end{array}$ & $\begin{array}{c}\text { Prepreg } \\
\text { Thickness per } \\
\text { Orientation } \\
\text { [mm] }\end{array}$ & $\begin{array}{c}\text { Total } \\
\text { Laminate } \\
\text { Thickness } \\
\text { [mm] }\end{array}$ \\
\hline GLARE3-5/4-0.3 & $0^{\circ} / 90^{\circ}$ & 0.33 & 0.125 & 2.65 \\
\hline GLARE3-4/3-0.3 & $0^{\circ} / 90^{\circ}$ & 0.33 & 0.125 & 2.07 \\
\hline GLARE4-3/2-0.3 & $90^{\circ} / 0^{\circ} / 90^{\circ}$ & 0.30 & 0.125 & 1.91 \\
\hline
\end{tabular}

In order to capture the local stress-strain behaviour surrounding the loading pin/hole, electrical resistance strain gages were bonded to the specimen at a predetermined location. The gages used were $120 \Omega$, HBM LY63-1.5/120 single axis gages for the quasi-isotropic specimens and $120 \Omega$, HBM XY93-1.5/120 biaxial, stacked gages for the orthotropic specimens, supplied by Durham Instruments (Pickering, Ontario, Canada). These gages were temperature compensated for bonding onto an aluminum substrate and their application included exhaustive surface preparation protocols as recommended by the manufacturer.

The location of the gages and selection of grid size warrants further justification and will be discussed next. The modified CCD FE study discussed in Chapter 2 analyzed the strain field surrounding the fastener hole. The resulting modified CCD represents a threshold beyond which any movement in the direction towards to the leading edge of the hole would result in stresses above the yield strength of the aluminum and vice versa in the opposite direction. Since the detection of both yielding and buckling are important to the experimental investigation of the modified CCD and YDB mechanism, values of the modified CCD calculated for each variant were rounded to the nearest millimetre resulting in a $2 \mathrm{~mm}$ distance from the $\mathrm{S}_{11}$ criterion and $4 \mathrm{~mm}$ for the combined stress 
criteria. It was at these distances that the gages were applied. For the quasi-isotropic cases, the $2 \mathrm{~mm}$ distance and single axis gages were employed while the orthotropic case, with its increased complexity, employed the $4 \mathrm{~mm}$ combined stress distance and biaxial strain gages. Examining both cases in this manner enabled a more complete investigation of the modified CCD and YDB mechanism. Figure 3.4 and Figure 3.5 show the bonded strain gages with their corresponding distances for both the quasi-isotropic and orthotropic specimens, while Figure 3.6 shows a quasi-isotropic specimen with the hardened steel loading pin inserted as part of the tension based loading fixture.

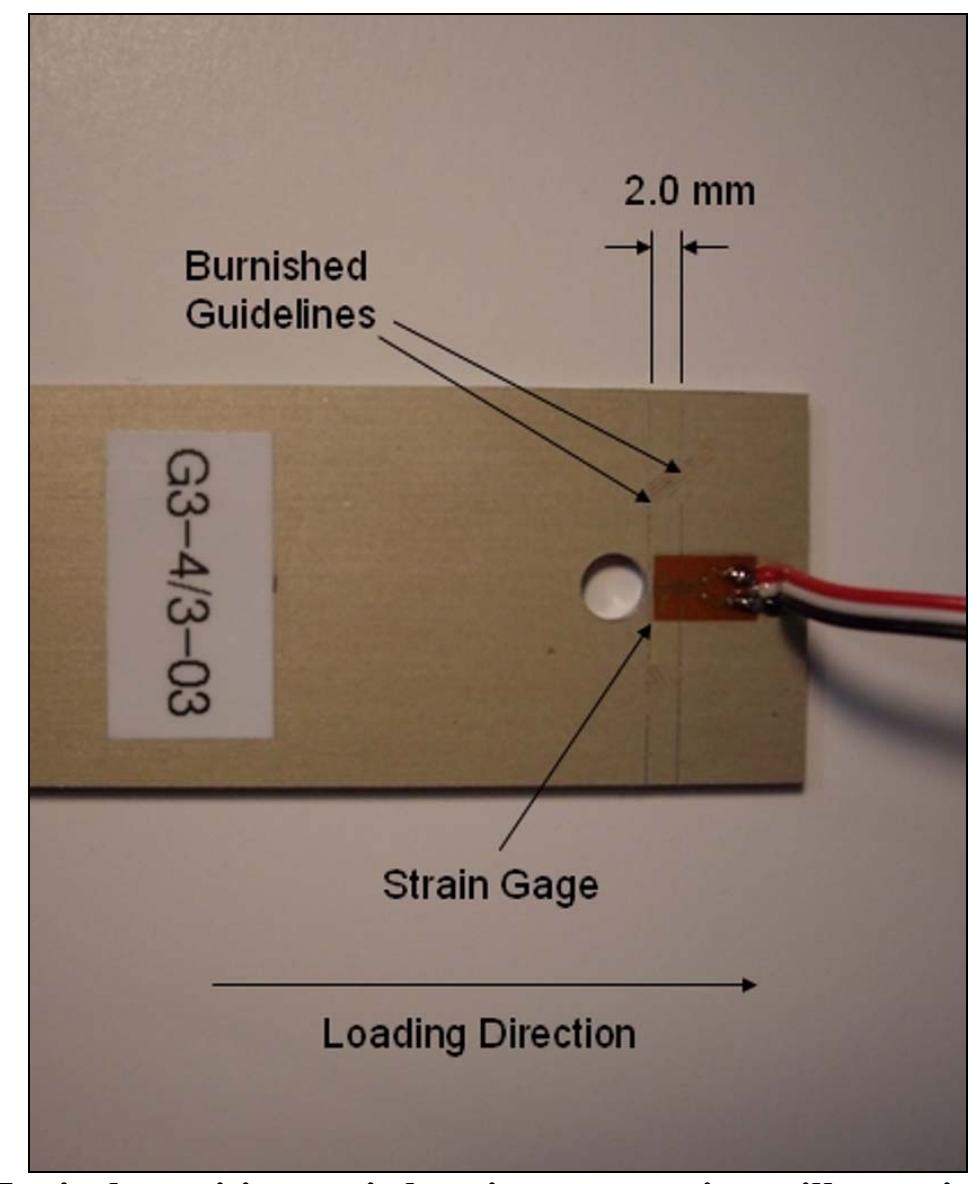

Figure 3.4: Typical quasi-isotropic bearing test specimen illustrating the location and orientation of the bonded strain gage. 


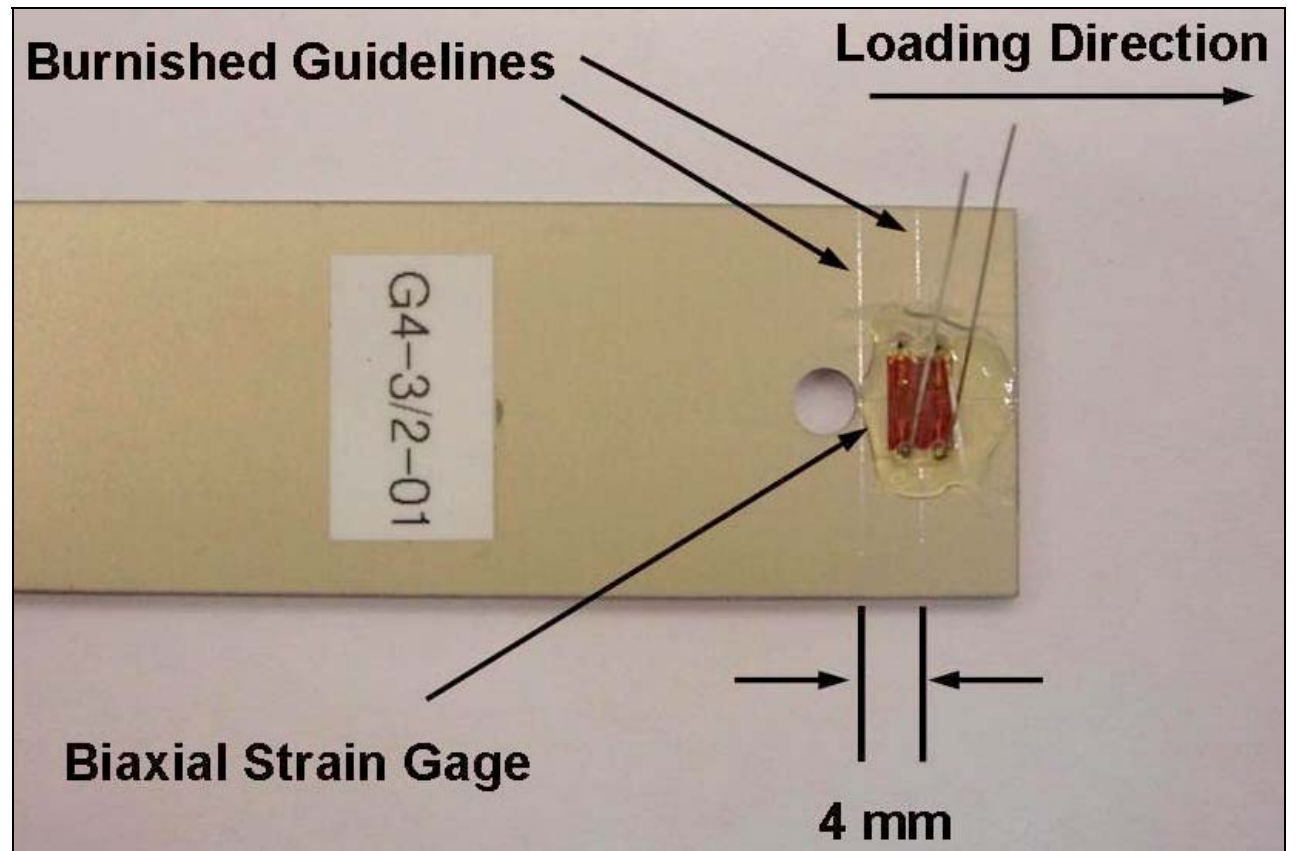

Figure 3.5: Typical orthotropic bearing test specimen with the location and orientation of the bonded strain gage shown.

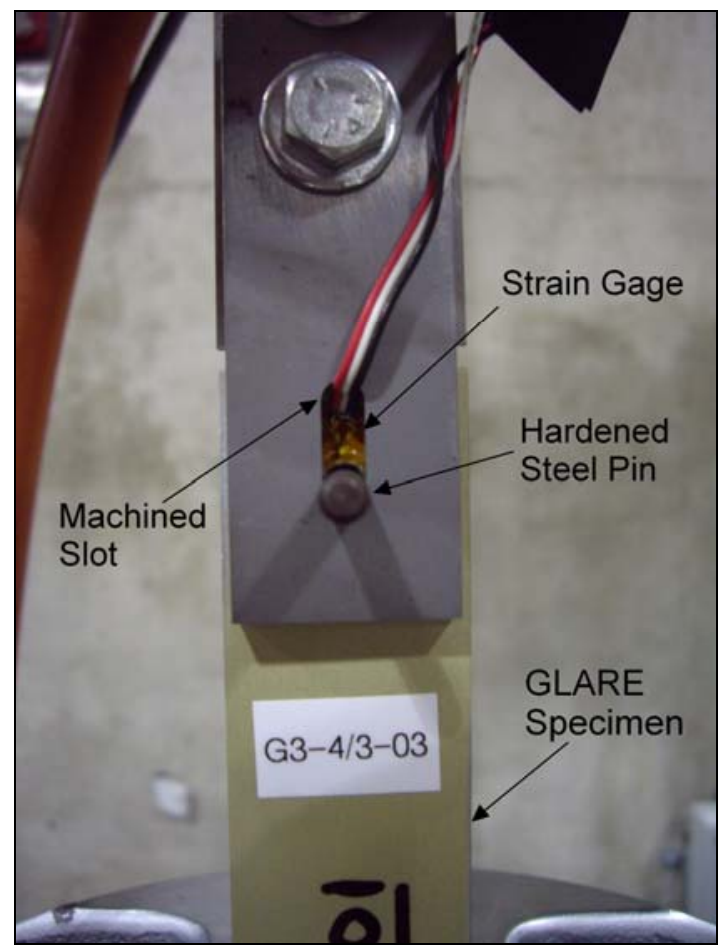

Figure 3.6: Typical quasi-isotropic bearing test specimen with the loading pin inserted as part of the tension based loading fixture. 
Furthermore, since one of the goals of this portion of the study is to extract and detect the occurrence of buckling and its proposed connection to the yielding of aluminum, it is important to place the location of the gage such that the centre of gage lies on the modified CCD. The orthotropic GLARE4 variant was chosen to be investigated in this case since the nature of its lay-up with increased $90^{\circ}$ plies makes it more susceptible to buckling. The use of the biaxial gage on these specimens will enable the detection of buckling through an analysis of their acquired data.

\subsubsection{Pin Bearing Experimental Protocol}

All experiments were carried out under ambient test conditions on a calibrated, MTS 322 test frame (MTS Systems Corporation, Eden Prairie, Minnesota, USA) under displacement control, with a $0.127 \mathrm{~mm} /$ Min loading rate. Applied loads were measured with both a $250 \mathrm{kN}$ and a $45 \mathrm{kN}$ load cell to ensure a $\pm 1 \%$ error compliance as stipulated by the standard. Adequate warm-up times for system hydraulics and analog circuitry was maintained for all tests to minimize any transient effects as a result of the previous shut down/start up.

A minimum of 5 specimens were tested under identical conditions and parameters for each variant of GLARE in order to establish a representative sample population. Actuator displacement was allowed to continue until the output from the load cell(s) began to significantly plateau after a maximum value was achieved, at which point the test was stopped. The motivation behind this stems from the fact that the relatively stiffer loading pin will plough its way continuously through the specimen material until a free edge is reached. Failure in a bearing mode and in particular the yielding/delamination buckling 
of the aluminum layers, occurs much earlier in the loading history and therefore any data captured long afterwards bears little significance to the scope of the study at hand. An MTS 634.12F-24 calibrated extensometer was applied on the quasi-isotropic specimens to indicate the movement of the free end of the specimen, relative to the loading pin within the test fixture. This served as a measurement of the test coupon's overall deformation and with the addition of the local measurement via the bonded strain gage, represents a comprehensive measure of the deformation within the specimen. Though the anticipated displacement for this loading configuration was quite small, the extensometer was mounted to the specimen and test fixture with dental rubber bands, providing adequate tension to avoid slippage during the test. Figure 3.7 depicts a typical setup, mid-test, with extensometer in place and strain gage wired for data acquisition. Multi-channel data for the strain gage, load cells, extensometer and onboard linear variable displacement transducer (LVDT) were acquired with custom built hardware/software as well as existing MTS hardware/software. Data was acquired at a rate of $25 \mathrm{~Hz}$, which compared to the relatively slow loading rate, allowed for a large amount of captured data points. This ensured that significant events during the loading history were adequately captured for analysis.

Outputs from the additional sensors - namely the $45 \mathrm{kN}$ load cell and bonded strain gages - were amplified and conditioned before integration into the MTS system, via analog inputs, to enable synchronized time stamping of the acquired data from all channels. Once bonded onto the test coupon, the strain gages were checked for continuity and resistance by a multi-meter to ensure no damage had occurred during the application process. A one-quarter Wheatstone bridge completion circuit was employed and 


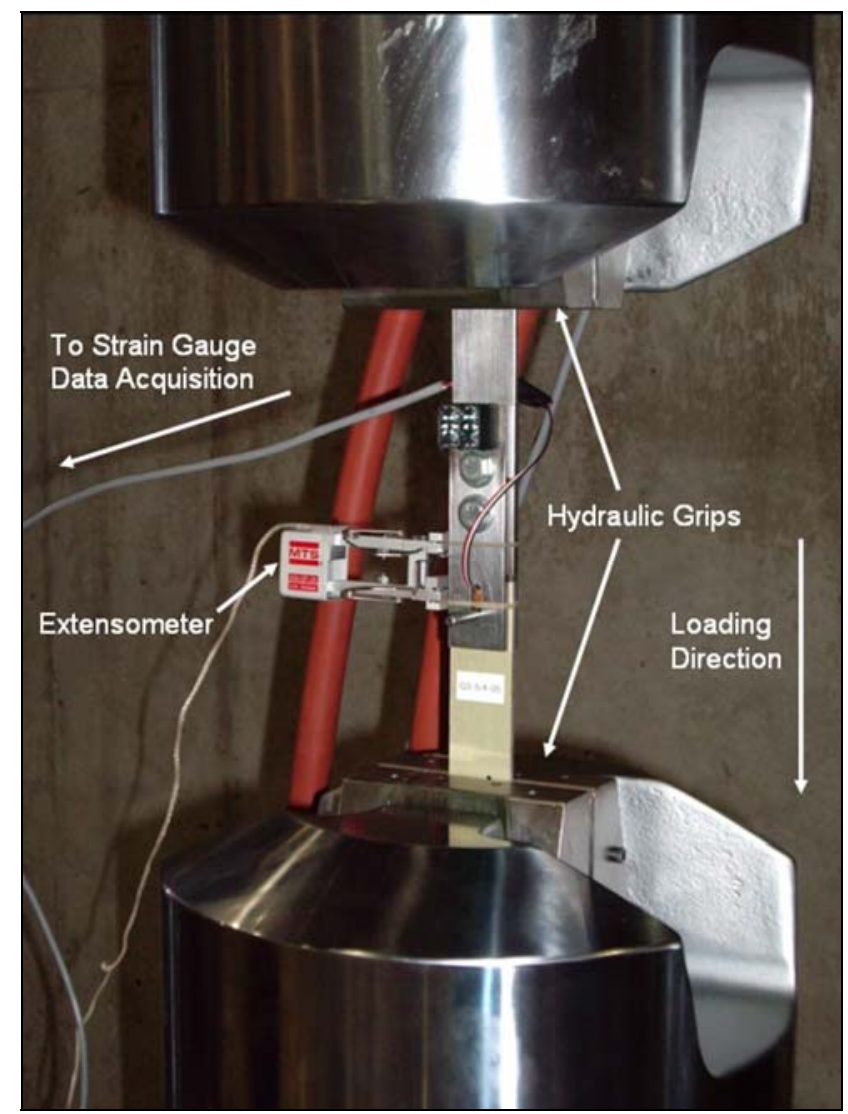

Figure 3.7: MTS 322 load frame with quasi-isotropic GLARE specimen mounted in the test fixture and extensometer in place for the measurement of relative pin displacement.

incorporated a three wire (26 AWG) configuration for temperature compensation and protection against de-sensitization of the gage through lead wire resistance. To further protect the integrity of the output signal, the length of the unshielded lead wires from the gage was kept to less than $150 \mathrm{~mm}$ at which point they were soldered to $24 \mathrm{AWG}$, individually shielded instrument wire. Gages and lead wire connections were protected from moisture ingress by applying two coats of polyurethane with a minimum 24 hour cure time between coats and before testing.

Bridge completion was accomplished via a BCM-1 bridge completion module (Omega, Laval, Quebec, Canada) while external excitation, analog signal conditioning and analog 
output scaled from 0 Volts to 10 Volts was provided by a DP25B-S strain gage panel meter (Omega, Laval, Quebec, Canada). This output scale was required for compatibility with the existing MTS data acquisition system.

The selection of bridge excitation voltage is subject to a number of constraints including power dissipation, substrate conductivity and gage resistance. A high value of excitation voltage allows for easier bridge balancing and a higher output voltage from the sensor but must be optimized since the relatively small area of the grid does not allow for efficient power dissipation. Overheating of the grid itself can occur at improperly assigned excitation voltages and will ultimately lead to erroneous strain measurement. The power density dissipated in the constantan grid in Watts per square meter can be calculated using Equation (1.4):

$$
P_{G}^{\prime}=\frac{E_{B}^{2}}{4 \cdot R_{G} \cdot l_{G} \cdot W_{G}}
$$

where $E_{B}$ is the bridge excitation voltage, $R_{G}$ is the nominal resistance of the gage, $l_{G}$ is the length of the grid and $\mathrm{W}_{\mathrm{G}}$ is the width of the grid. Recognizing that 2024-T3 aluminum as a substrate is an excellent heat sink we can assign a maximum power density dissipation value of $7500 \mathrm{~W} / \mathrm{m}^{2}$. Rearranging Equation (1.4) to solve for excitation voltage yields a value of 2.324 V. Practical application of this excitation value into the system involved a voltage division circuit in series with the external excitation provided by the strain gage panel meter and a final, high precision potentiometer to further reduce the voltage to the desired value. 


\subsection{Results and Discussion of the Pin Bearing Experiments}

In the following sections, the results from the bearing strength experiments performed on GLARE3-5/4-0.3, GLARE3-4/3-0.3 and GLARE4-3/2-0.3 will be presented. A presentation and discussion of the extraction and interpretation of traditional bearing yield strength values as well as newly defined ones is also included. This discussion will also justify the extensions to the current test standards, as part of a more in depth examination of a pin bearing loading configuration. Finally, the detection of buckling and its relationship to the defined bearing yield strength, by virtue of the acquired strain profiles, will also be discussed.

\subsubsection{Examination of the Failed Specimens}

Some of the tested specimens were photographed under magnification to examine the nature of the deformation, in a bearing failure mode. Figure 3.8 depicts a typical quasiisotropic specimen having failed in this manner. It is evident from the figure that the fastener hole has elongated a substantial amount, which is indicative of bearing failure. This deformation, some of which is out of plane, is coupled with delamination and yielding of the aluminum layers, as expected from the enforced pin bearing configuration. The clearly visible yielding, delamination and buckling of the material directly adjacent to and moving away from the specimen hole is noteworthy since it provides empirical support for the previous analytical result, which predicted precisely this behaviour. Figure 3.9 and Figure 3.10 show two typically failed orthotropic specimens, showing substantial portions with yielding, delamination and buckling as predicted by the 


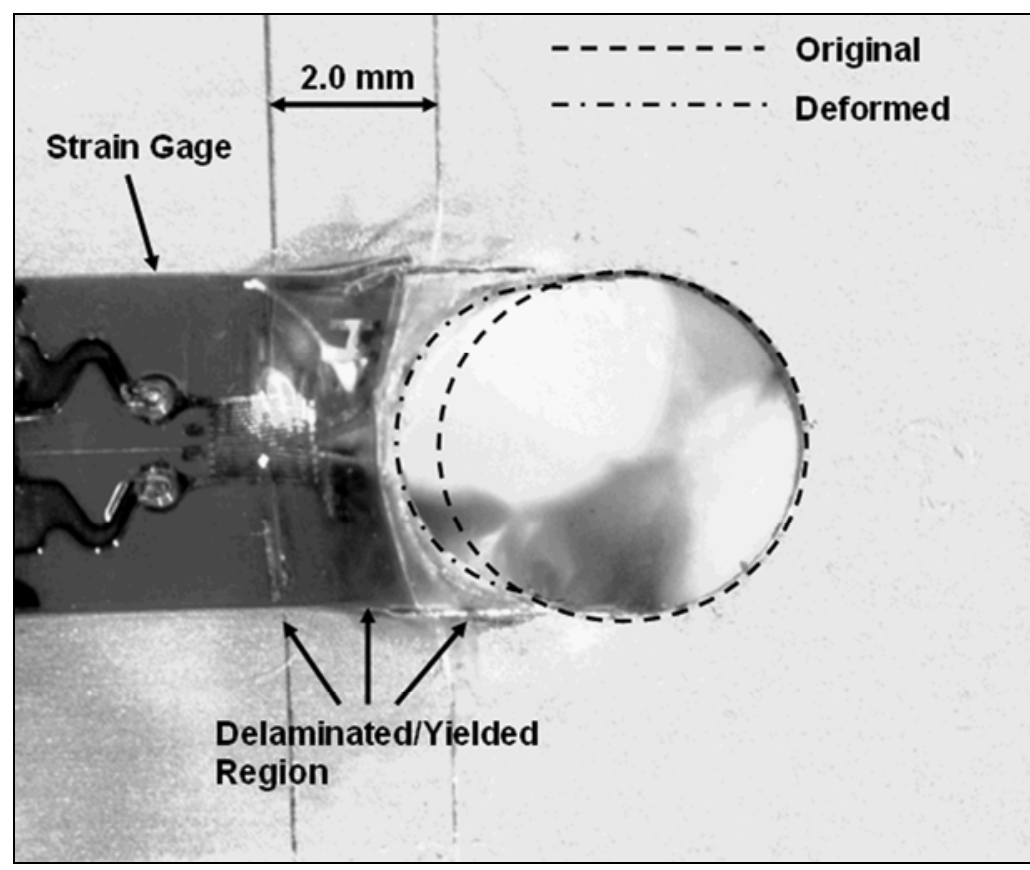

Figure 3.8: Typical quasi-isotropic specimen failing in a bearing mode. Note the elongation of the fastener hole and the significant amount of delamination/yielding, some of which is out of plane.

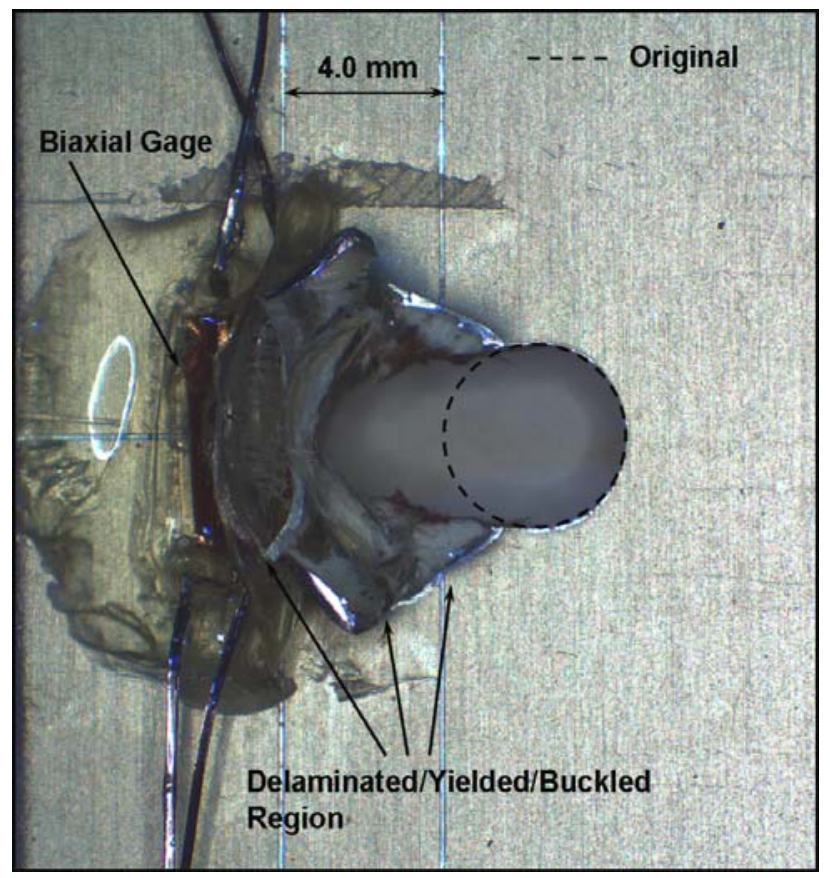

Figure 3.9: Typical failed specimen of GLARE4 exhibiting an elongated hole with extensive delamination, yielding and buckling clearly visible. 


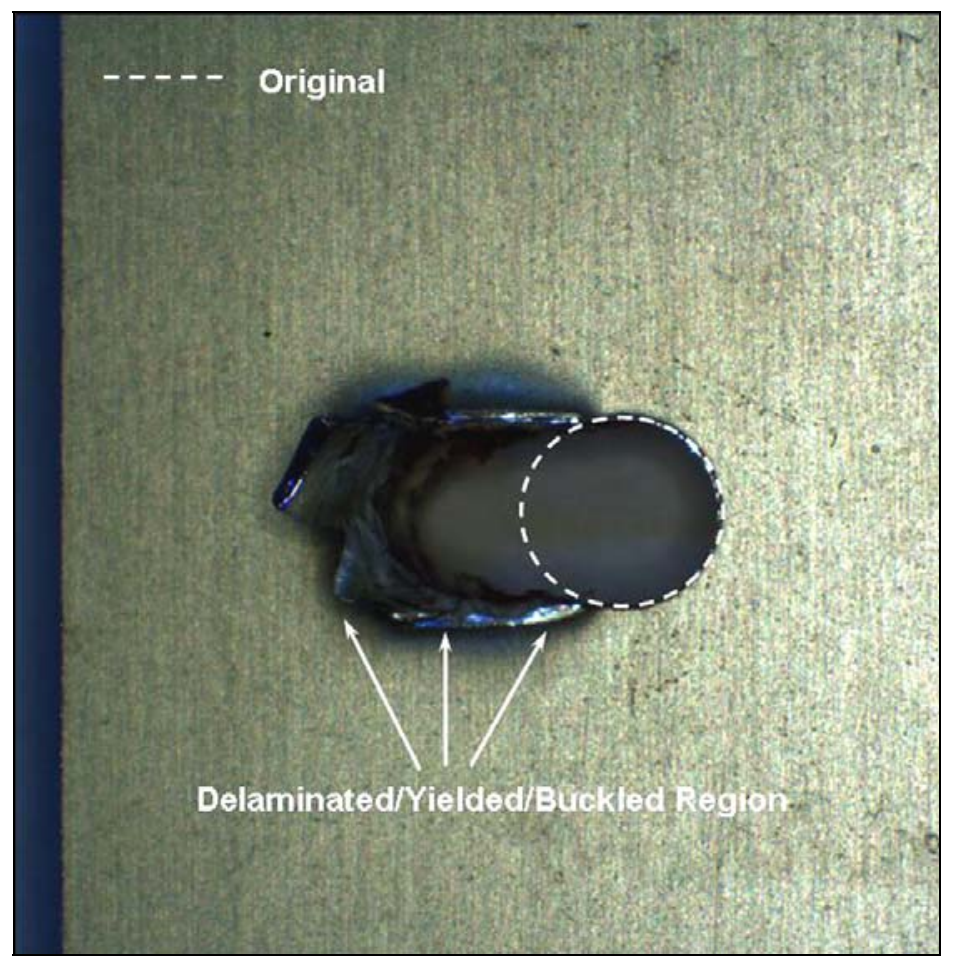

Figure 3.10: Typical failed specimen of GLARE4 from a reverse perspective exhibiting an elongated hole with extensive delamination, yielding and buckling clearly visible.

analytical results and in accordance with the YDB mechanism. The additional damage in the orthotropic specimens is symptomatic of their variant and lay-up with additional transverse plies making them more prone to buckling, as anticipated.

\subsubsection{Results of the Globally Applied Measurements}

Data acquired from the tested specimens was imported into and analyzed by Maple V10 software [114] using custom written routines. Raw data was filtered using an 11 point moving average algorithm to smooth out local discontinuities and inherent fluctuations, typical of analog signals. Figure 3.11 and Figure 3.12 depict the bearing load versus extensometer displacement curves for all of the GLARE3-5/4-0.3 and 


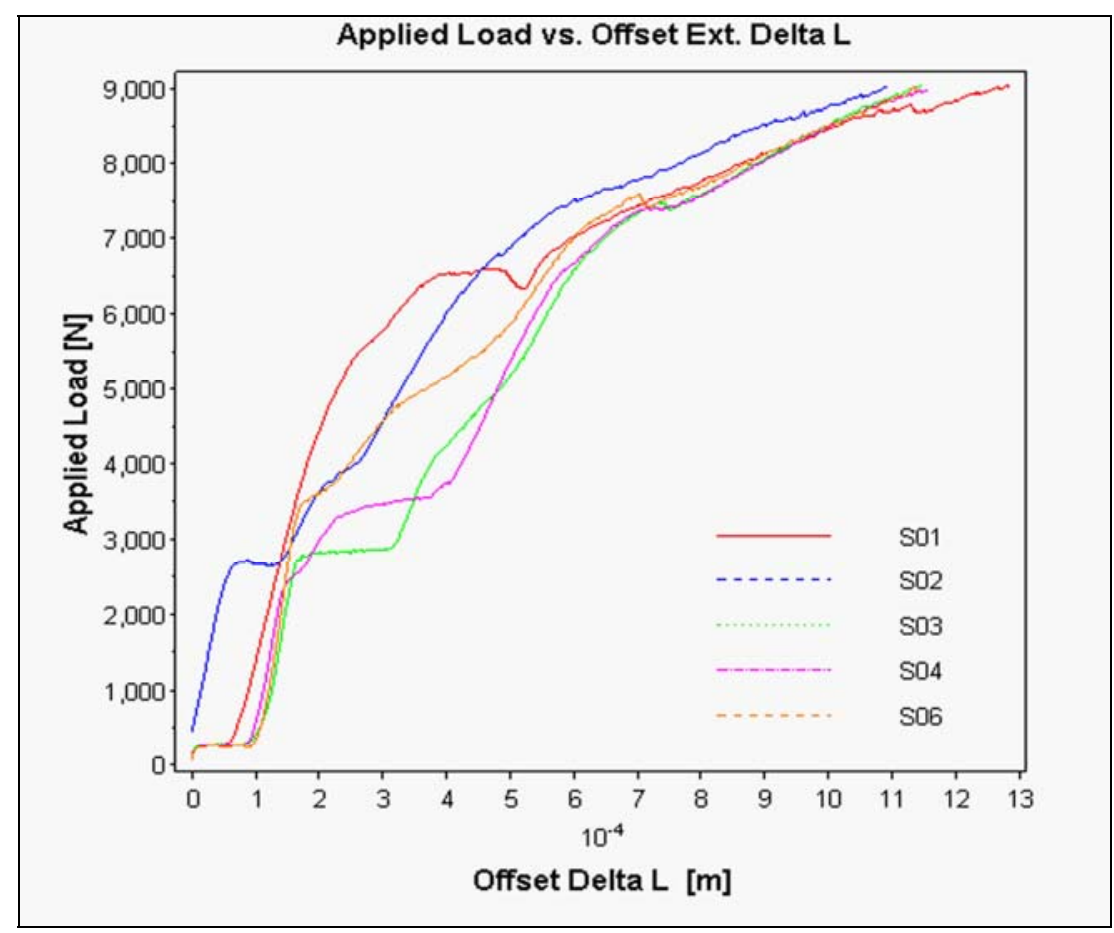

Figure 3.11: Bearing load versus extensometer deformation curves for GLARE35/4-0.3.

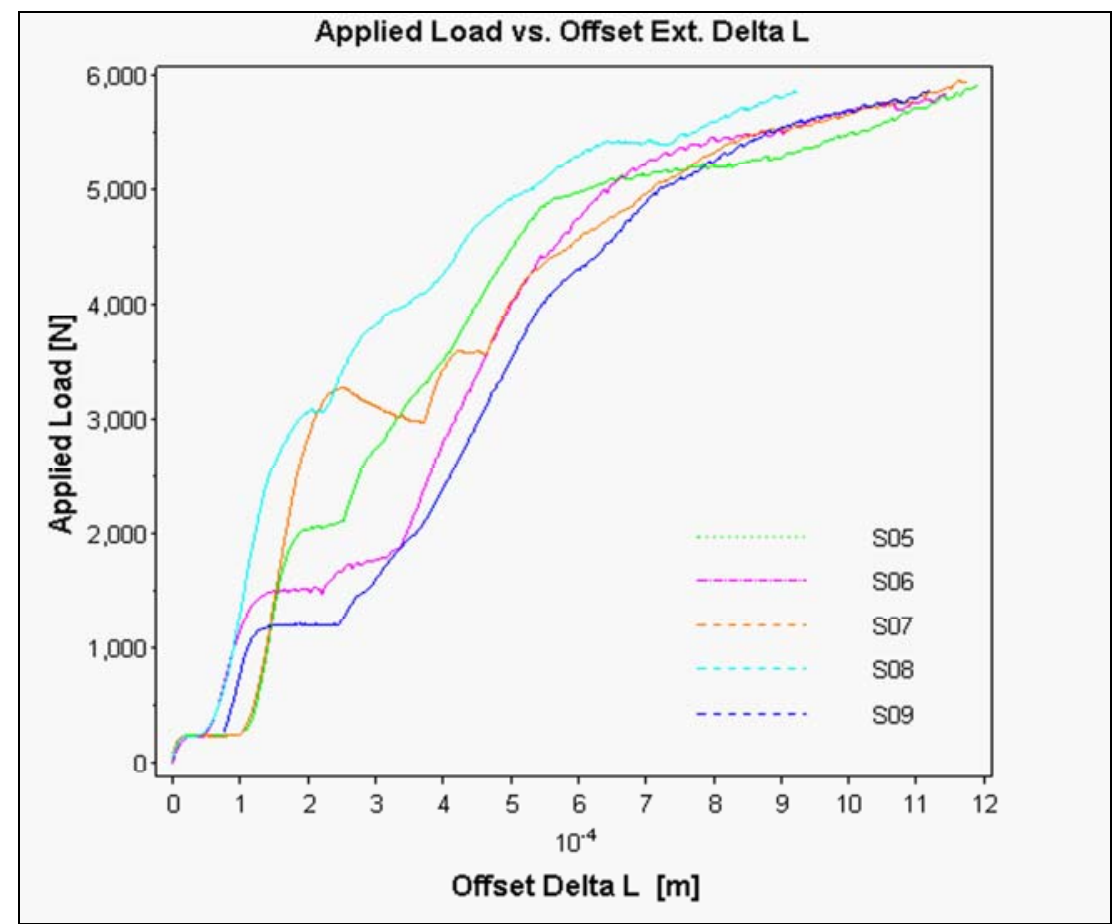

Figure 3.12: Bearing load versus extensometer deformation curves for GLARE34/3-0.3. 
GLARE3-4/3-0.3 specimens tested. These represent the standard load - deformation curves as specified by ASTM D953-02 [68]. An examination of the load-deformation curves shows that the deformation axis continuously increases and does not exhibit any sudden drops throughout the test window. This suggests that extensometer slippage did not occur during the tests and that the addition of dental rubber bands to secure the extensometer in place was effective.

While there is much merit to the generation of these profiles for bearing behaviour, for the purposes of this investigation a global measurement scheme such as this is not sufficient for the examination and subsequent calculation of yield at the modified CCD. Such an analysis requires a local perspective of the strain behaviour in addition to the global one, which is why the bonded strain gages were applied in this investigation.

\subsubsection{Calculation of Standard Bearing Yield Strength}

Examining the data from the above curves, the precise bearing load at $4 \%$ permanent deformation of the fastener hole can be extracted. This definition relates the load applied to a value at which the fastener hole is deformed a given percentage of its own diameter. By virtue of this definition, we see that it is subject to zero-load errors. This inherent error was eliminated by offsetting the extensometer values such that the initial recorded value (greater than zero) corresponded to a zero displacement, at near zero load values. This of course is within the limits of the accuracy provided by the $45 \mathrm{kN}$ load cell and represents an error much less than $1 \%$.

With all of the tested specimens containing a hole of $4.65 \mathrm{~mm}$ in diameter, the associated value on the extensometer deformation axis equal to $4 \%$ of this was $0.186 \mathrm{~mm}$. Taking 
the applied load value corresponding to a $0.186 \mathrm{~mm}$ displacement will yield the standard bearing load for that specific specimen. The bearing strength in MPa can then be calculated via the previously discussed Equation (1.3) repeated here for clarity:

$$
\sigma_{b}=\frac{P}{D t}
$$

where $P$ is the bearing load, $D$ is the fastener hole diameter and $t$ is the specimen thickness. Table 3.2 summarizes the arithmetic mean of each set of bearing loads calculated, the standard deviation of the observations and the associated bearing yield strength values.

Table 3.2: Summary of the mean bearing loads, standard deviation and associated bearing yield strength, corresponding to a $4 \%$ permanent hole deformation.

\begin{tabular}{cccc}
\hline GLARE Variant & $\begin{array}{c}\text { Mean Bearing Load } \\
\text { [N] }\end{array}$ & $\begin{array}{c}\text { Standard Deviation } \\
\text { [N] }\end{array}$ & $\begin{array}{c}\text { Bearing Yield } \\
\text { Strength [MPa] }\end{array}$ \\
\hline GLARE3-5/4-0.3 & 3348.758 & 567.731 & 271.760 \\
\hline GLARE3-4/3-0.3 & 2357.669 & 911.236 & 244.940 \\
\hline
\end{tabular}

\subsubsection{Results of the Locally Applied Measurements}

The results presented in the preceding section are in accordance with standard test protocols and values of bearing yield strength were extracted accordingly. For the purposes of this investigation however, it is important to investigate the incidence of yield at the modified CCD as part of the YDB mechanism and the practical progressive damage methodology. The inclusion of the bonded strain gage is well suited for such an investigation since its location - at the modified CCD - allows for the measurement of 
strain and ultimately yield of the aluminum at that precise location. Figure 3.13 and Figure 3.14 depict the bearing stress as calculated by Equation (1.3) versus the measured output from the bonded strain gage for the quasi-isotropic specimens. These acquired profiles can now be characterized for the detection of bearing yield.

In addition, Figure 3.15 and Figure 3.16 show the bearing stress versus the measured strain in the longitudinal direction of all the GLARE4, orthotropic specimens tested.

These profiles were split into separate figures for the sake of clarity alone. An examination of these curves shows a very similar initial behaviour but also introduces buckling behaviour in the higher strain regimes.

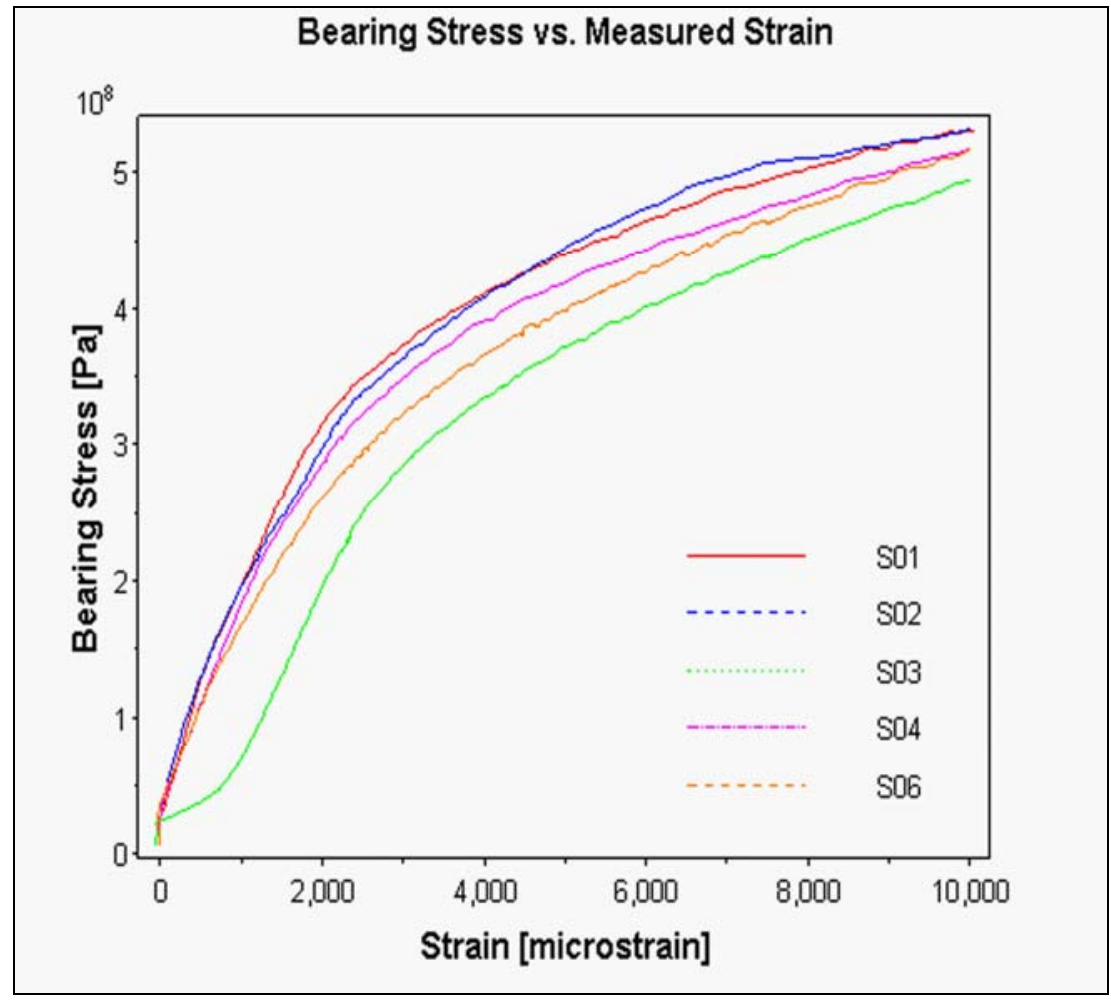

Figure 3.13: Calculated bearing stress versus measured strain curves for GLARE35/4-0.3. 


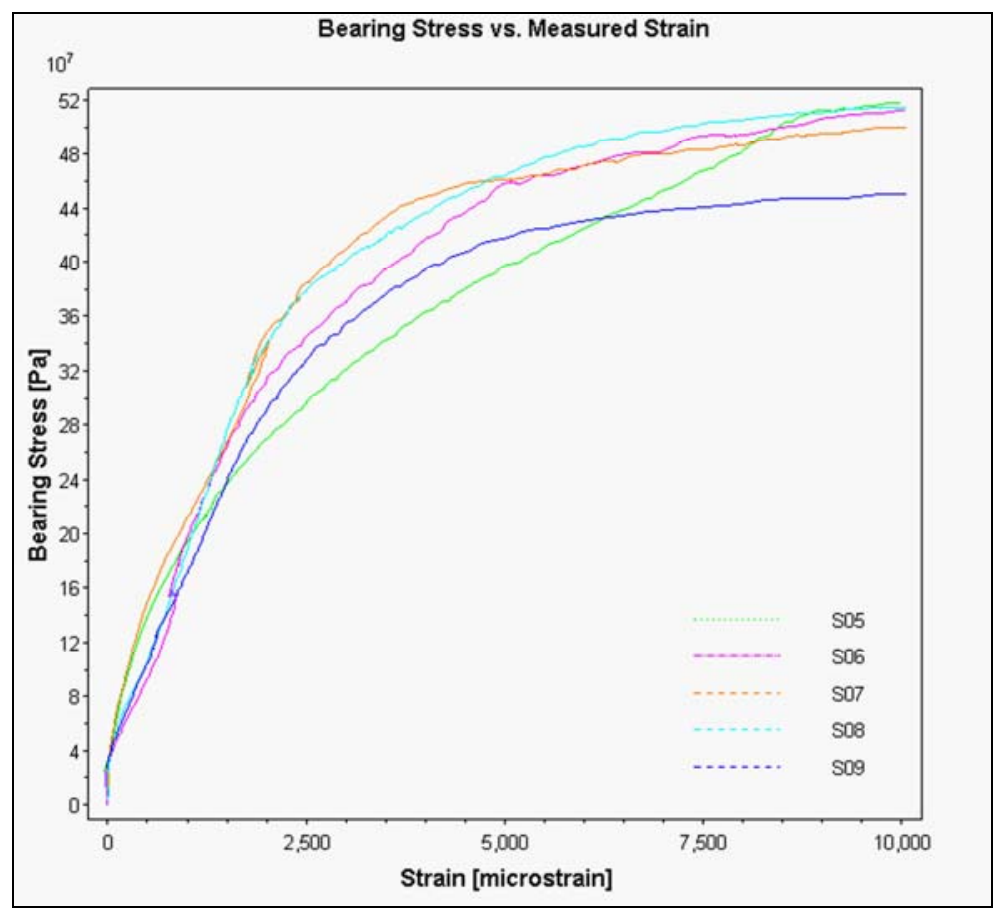

Figure 3.14: Calculated bearing stress versus measured strain curves for GLARE34/3-0.3.

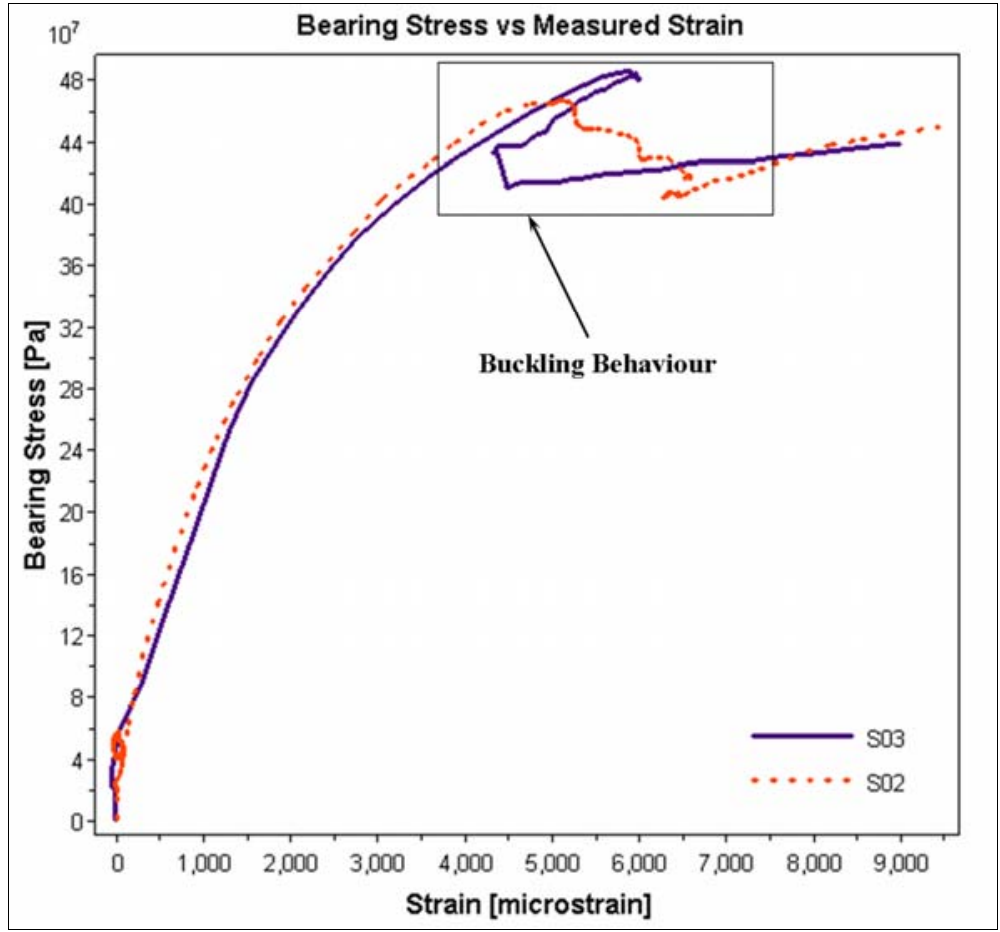

Figure 3.15: Calculated bearing stress versus measured longitudinal strain curves for GLARE4-3/2-0.3. 


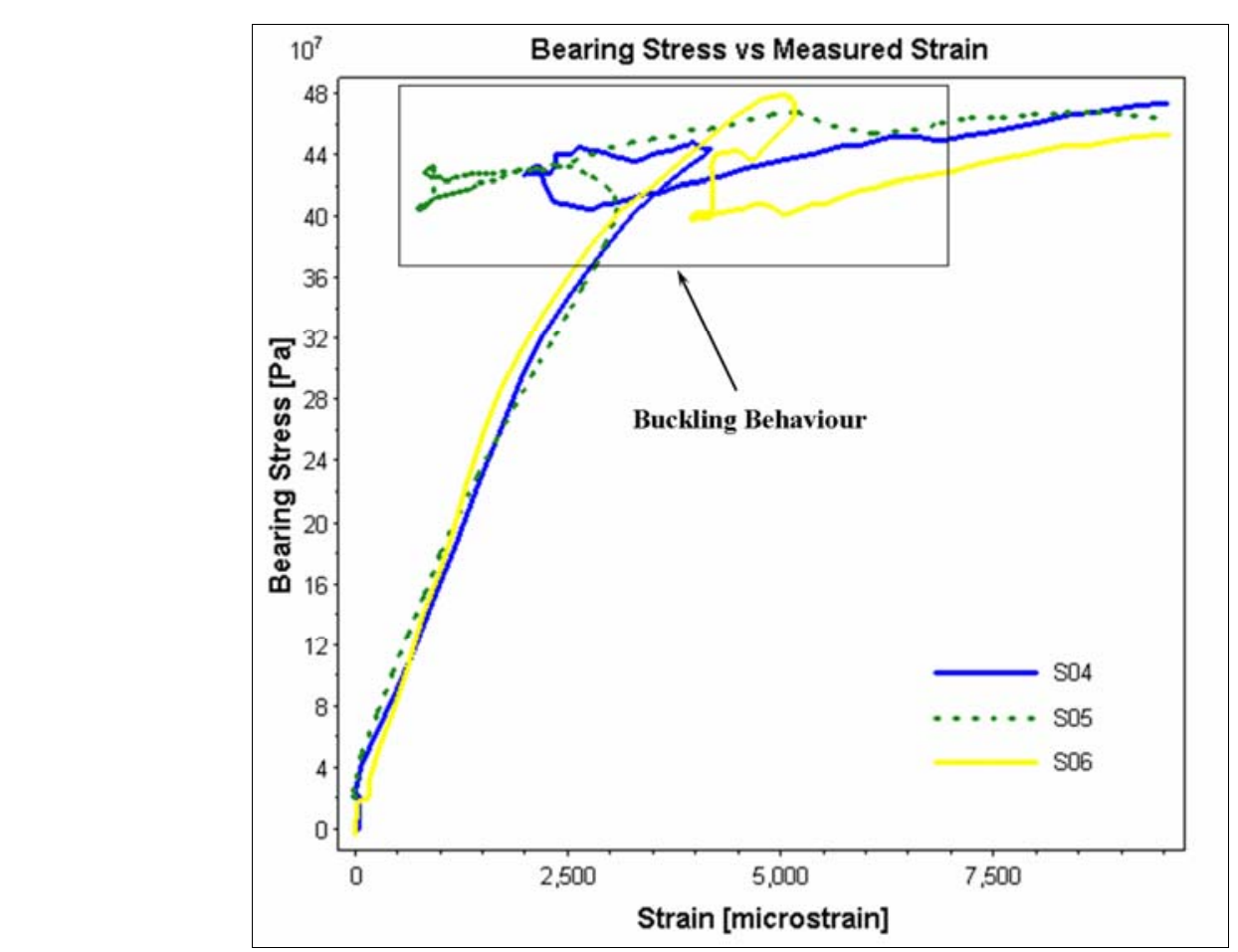

Figure 3.16: Calculated bearing stress versus measured longitudinal strain curves for GLARE4-3/2-0.3.

The detection of buckling in the aluminum layers is also important to the experimental investigation of the YDB mechanism. This behaviour was anticipated and is the reason why the biaxial gages were applied to the GLARE4 variants which were exceptionally prone to buckling. An analysis of this observed buckling behaviour and its connection to the yielding of the aluminum will be presented in subsequent sections of this chapter.

\subsubsection{Characterization of the Locally Measured Bearing Stress-Strain Curves}

In order to examine yield at the modified CCD as part of the YDB mechanism, we must investigate the nature of the generated bearing stress versus measured strain curves $\left(\sigma_{b}-\varepsilon\right)$ first. At first glance, the generated $\sigma_{b}-\varepsilon$ curves resemble a bilinear response, with linear 
portions at both the low and high strain regimes. This is entirely analogous to the curves generated by a uniaxial tensile test for which a bilinear approximation remains as the generally accepted model [27]. This approach employs a constant stiffness for the elastic (low strain) and plastic (high strain) portions of the curve and approximates the nonlinear portion by extending these linear regions to create an idealized bilinear response [27]. The yield strength of the laminate was then defined as the so-called "knee-point" of the two linear portions - in other words, at their intersection. In general, this procedure has been shown to be effective in calculating laminate ultimate strength but it is somewhat of an oversimplification with regards to its yield definition since it doesn't include the non-linear bridge connecting the low and high strain portions. A more comprehensive definition of yield should include the behaviour of this non-linear portion and that is precisely what is proposed herein.

Figure 3.17 depicts a typical $\sigma_{b}-\varepsilon$ curve for a GLARE3-4/3-0.3 specimen focusing on strain values below $10,000 \mu \varepsilon$ and also shows both the low strain and high strain linear approximations. The low strain and high strain portions of the curve have been linearly interpolated, via a minimization of the least squares error, whose output resembles the familiar slope y-intercept form of Equation (3.2):

$$
y=m x+b
$$

This linear model can be reinterpreted for our particular set of axes in the manner shown by Equation (3.3) where $\varepsilon$ is strain given in the dimensionless units of $\mu \varepsilon$ :

$$
\sigma_{b}=m \varepsilon+b
$$




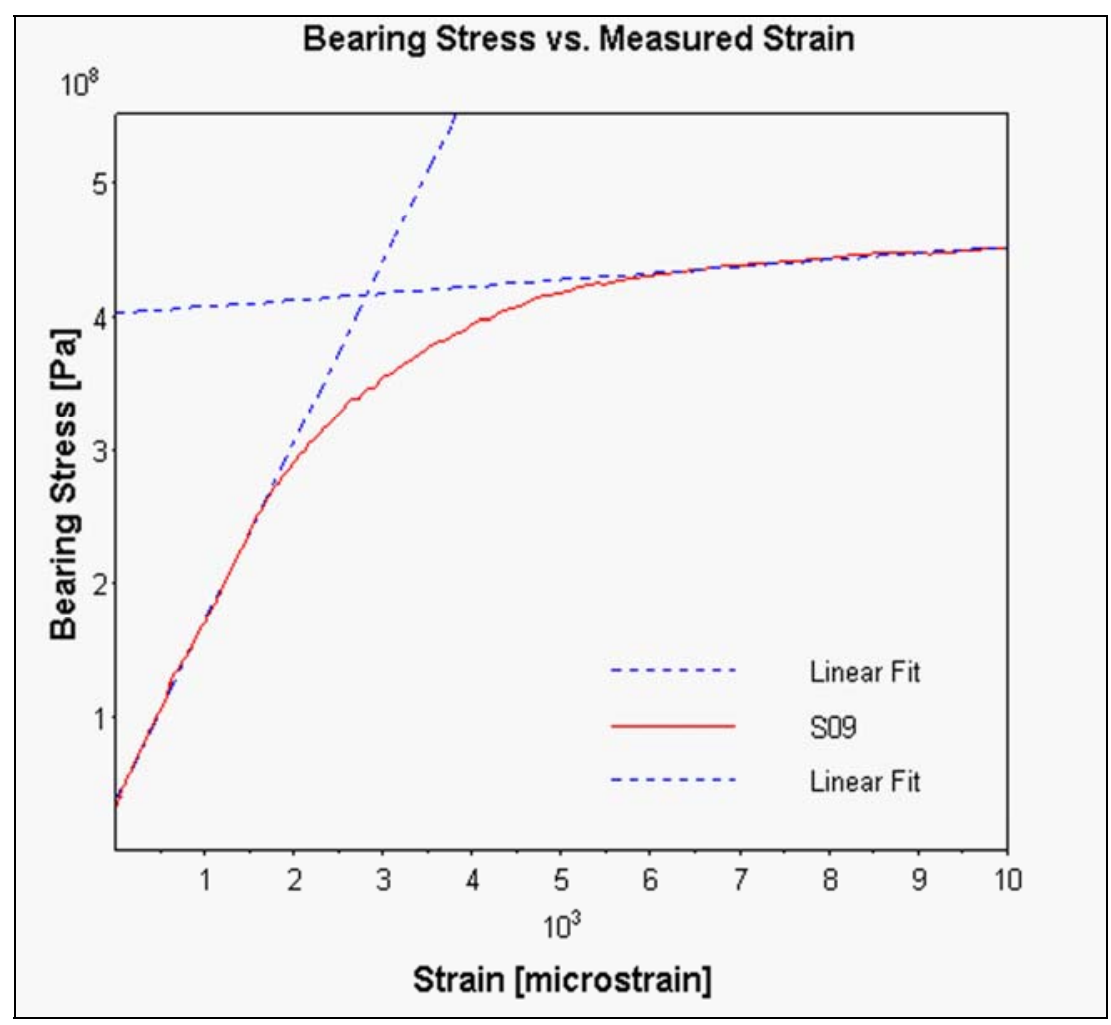

Figure 3.17: Bearing stress vs. measured strain curve for a GLARE3-4/3-0.3 specimen showing the linear approximations to the low and high strain regimes.

The linear functions generated for the low and high strain portions differ only by their associated slope and y-intercept values, which are empirically-based values as a result of the interpolation of the data. With respect to the low strain (elastic) interpolation the associated slope $m$ is in reality the laminate's elastic stiffness in response to a pin bearing load - an $E_{b}$ elastic to some extent. The same can be said for the linear function interpolated over the high strain region as well, except its implication refers to a plastic stiffness or secondary modulus.

It would not be valid to declare the former result (the $E_{b}$ elastic) as a measure of the laminate's overall elastic stiffness, particularly in response to a uniaxial tensile load, since it was derived by an empirical setup employing significantly different geometries 
and load configurations. However, the resultant shapes of the acquired $\sigma_{b}-\varepsilon$ profiles do enable the drawing of an appropriate analogy for the analysis.

The material's response to the bearing load can be explained in terms of its constituents. As a consequence of their relatively higher stiffness, the aluminum layers within the laminate attract the majority of stress and the initial loading resembles that of monolithic aluminum with a reduced elastic stiffness. As the load increases, the aluminum begins to locally yield giving rise to the non-linear response. By virtue of the epoxy matrix bonding the remaining laminate together, it begins to transfer its load to the adjacent glass layers but this quickly leads to delamination and subsequent buckling. The glass layers, which are brittle in nature and display limited plasticity, will carry a portion of this load until the delamination reaches a critical length thereby leaving them laterally unsupported and susceptible to buckling as well. Continued loading results in increased plastic deformation of the aluminum layers but since their compliance is greatly compromised, they continually transfer load to the glass layers leading to additional delamination and buckling. Final fracture of the specimen comes by way of the essentially rigid steel pin carving its way through the remaining material until it reaches a free surface.

That being said, the resulting linear interpolations mirror the low and high strain regimes effectively and intersect - as expected - but defining their intersection point as the bearing yield strength of the material overestimates the yield strength of the material leading to non-conservative values. 
An examination of the non-linear region reveals that it closely resembles a natural logarithmic function (hereby referred to as simply 'logarithmic') of the form shown in Equation (3.4) where once again $\varepsilon$ is the strain given in the dimensionless units of $\mu \varepsilon$. :

$$
\sigma_{b}=-a+b \ln (\varepsilon)
$$

The parameters $a$ and $b$ are empirically derived values as a result of the logarithmic fit, through a minimization of the least squares error, leading to an accurate depiction of the material's response over the intermediate interval. Figure 3.18 is a $\sigma_{b}-\varepsilon$ plot focusing on the non-linear portion of the full curve in the range of $2000 \mu \varepsilon$ to $6000 \mu \varepsilon$ to illustrate the achieved correlation between the acquired curve and its subsequent approximation.

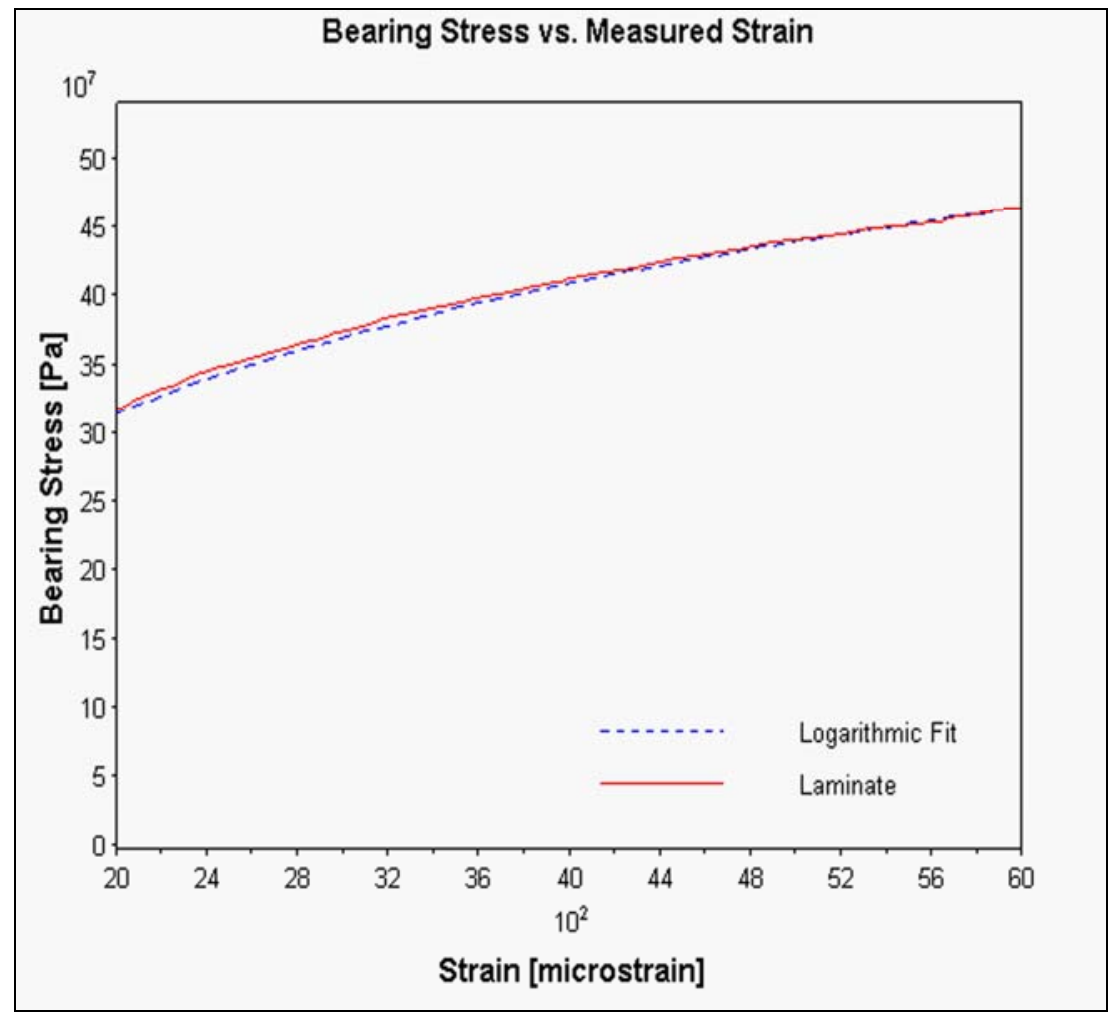

Figure 3.18: Non-linear portion of the bearing stress vs. measured strain curve for a GLARE3-5/4-0.3 specimen showing the logarithmic approximation. 
The final result of the curve fitting and linear approximation schemes employed can be summarized in a single, piecewise function acting over intervals as shown in Equation (3.5) where the subscripts $e l$, nonlin and $p l$ refer to the elastic, nonlinear bridge and plastic regions respectively:

$$
\sigma_{b}=\left\{\begin{array}{cc}
m_{e l} \varepsilon+b_{e l} & 0 \leq \varepsilon \times 10^{-6} \leq \varepsilon_{\text {nonlin }}^{e l} \\
-a+b_{\text {nonlin }} \ln (\varepsilon) & \varepsilon_{\text {nonlin }}^{e l} \leq \varepsilon \times 10^{-6} \leq \varepsilon_{\text {nonlin }}^{p l} \\
m_{p l} \varepsilon+b_{p l} & \varepsilon_{\text {nonlin }}^{p l} \leq \varepsilon \times 10^{-6} \leq \varepsilon_{\max }
\end{array}\right.
$$

The intervals for each portion of the piecewise function were selected such that the linear approximations were nearly tangent (and in some cases perfectly) to the logarithmic function at the endpoints of its effective domain. This is a useful property which is visibly tangible (particularly in Figure 3.17) and will be discussed in the next section. The range of the non-linear portion and its accompanying logarithmic approximation changed slightly from specimen to specimen but never differed more than $10 \%$. This relatively small error suggests constancy among the specimens.

Figure 3.19 and Figure 3.20 depict a direct comparison between the acquired data and the subsequent piecewise characterization for both a quasi-isotropic and an orthotropic specimen. Note that it is not possible to approximate the buckled region with the piecewise function and thus it is not included. This did not have a detrimental effect on the extraction of bearing yield strength as will be discussed in the next section. 


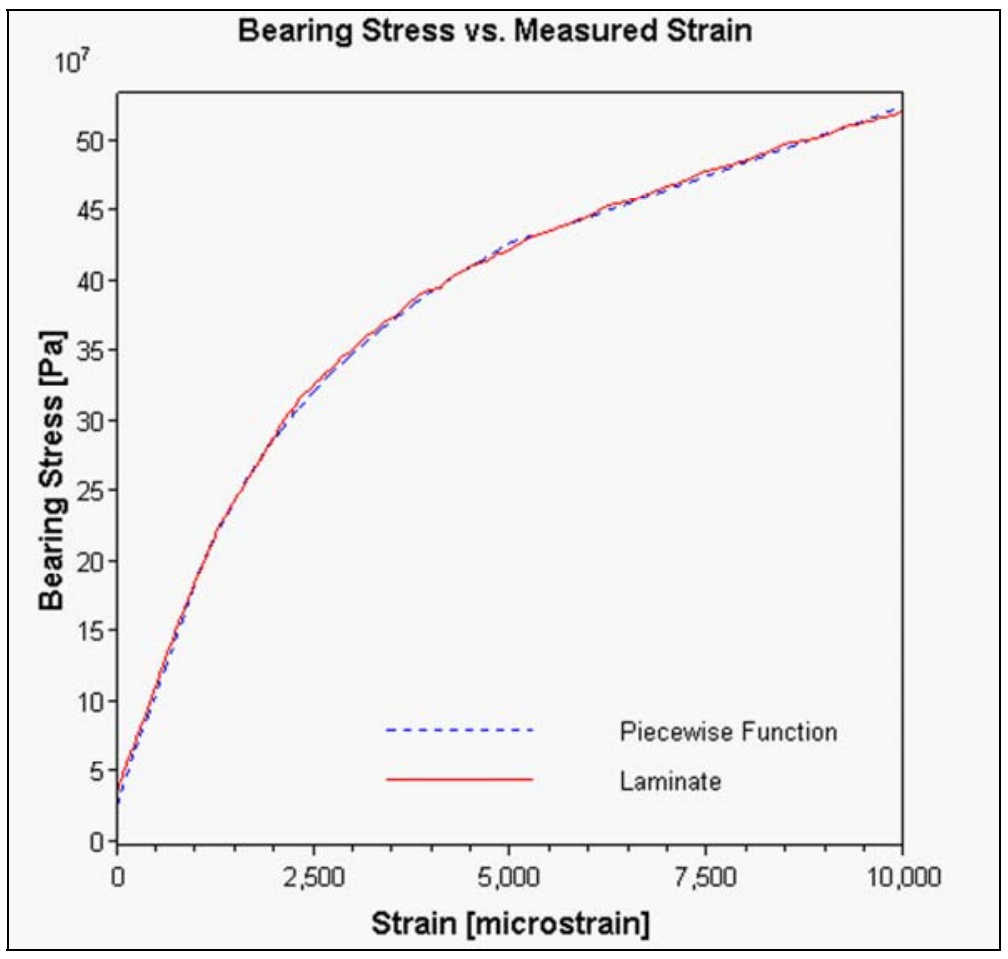

Figure 3.19: Bearing stress vs. measured strain curve for a GLARE3-4/3-0.3 specimen showing the piecewise characterization.

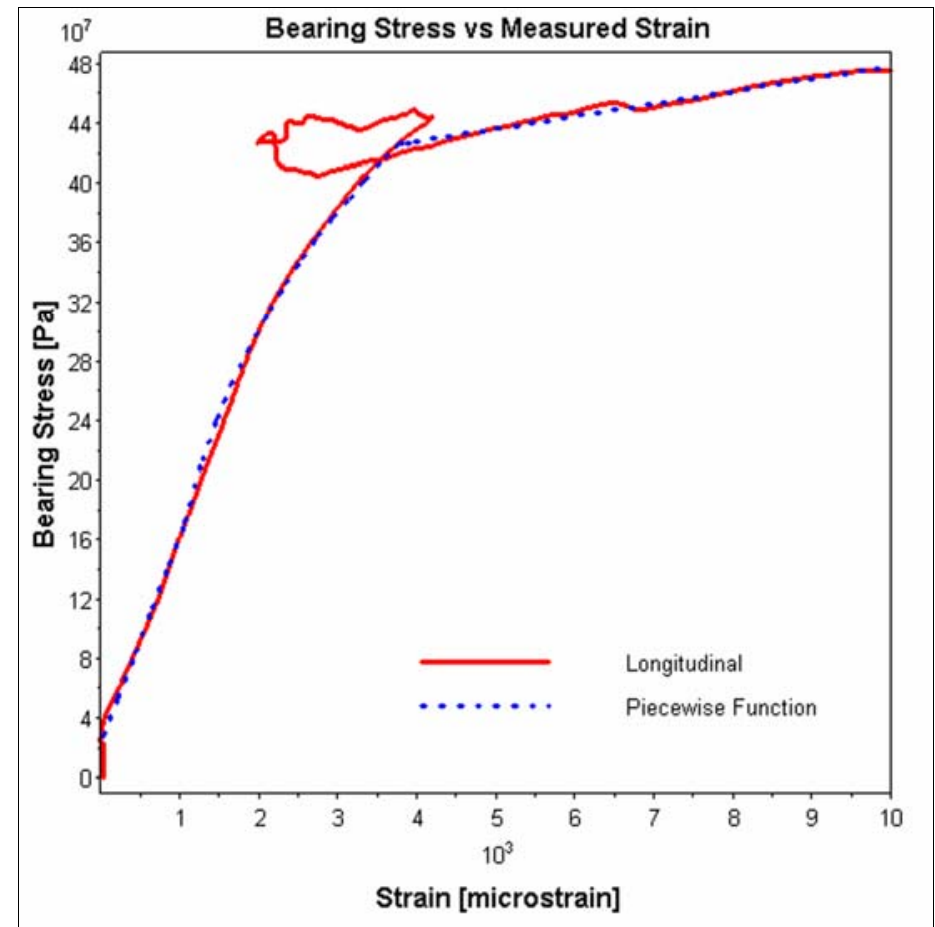

Figure 3.20: Bearing stress vs. measured strain curve for a GLARE4-3/2-0.3 specimen showing the piecewise characterization. 


\subsubsection{Calculation of a Locally Measured Bearing Yield Strength}

With a characterization of material response, a definition of a locally measured bearing yield including aspects from the entire curve can be proposed.

Analogous to the idealized bilinear approximation, the knee-point of the of the low and high strain linear portions was calculated at their intersection, near the $2000 \mu \varepsilon$ mark for most of the specimens tested. In order to place the yield point back onto the curve and produce realistic values, it is proposed that this intersection point be projected onto the non-linear segment, thus enforcing influence from all three of the curve's portions. This projected point on the $\sigma_{b}-\varepsilon$ curve's non-linear portion is the newly defined bearing yield strength.

This yield strength can be calculated as follows. By virtue of the curve geometry and due to the tangency of the lines at the endpoints of the non-linear portion and the continuity of the logarithmic function employed over that portion, a line which bisects the angle between the extended linear portions of the curve (through the knee-point) will intersect the logarithmic function at an angle of $\pi / 2$ radians. This second intersection point is the desired projection onto the non-linear curve and defined as the new bearing yield strength. For simplicity, this definition of yield will be called 'the bisection protocol'. Due to small variations in the acquired signal, not all of the linear interpolations for all the specimens were perfectly tangent to the logarithmic function at the endpoints of its domain and thus not all of the intersections were perfectly orthogonal. Although at a minimum, this deviation is difficult to eliminate entirely but still enabled near tangency to exist for all specimens and the offset can be considered inappreciably small. 
Though the decision to equally bisect - rather than unequally bisect - the linear approximations may be somewhat arbitrary, it is less so than defining yield via the employment of an offset technique with an arbitrarily assigned value of $0.2 \%$. Though familiar, such offset techniques receive influence from only the linear elastic portion of the curve rather than all of it. In addition, an equal bisection is the simplest means for projecting the knee-point of the two linear approximations onto the non-linear portion of the curve and lends itself to ease of application by virtue of the associated simple geometry.

The bisection protocol, as applied a quasi-isotropic GLARE3-5/4-0.3 specimen is shown in Figure 3.21 with a magnified view to illustrate the near perfect orthogonality of the intersection between the bisection line and the corresponding $\sigma_{b}-\varepsilon$ curve/logarithmic approximation.

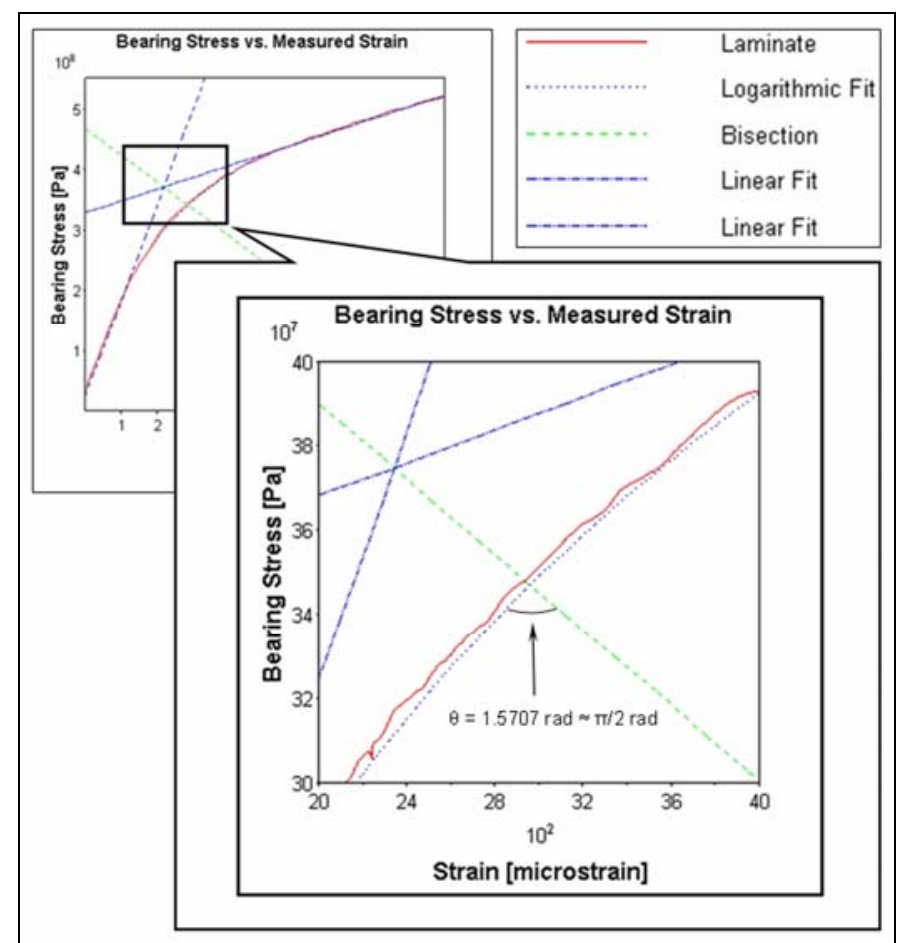

Figure 3.21: The bisection protocol as applied to a quasi-isotropic GLARE3-5/4-0.3 specimen. The magnified view shows the near-orthogonality of the intersection. 
The calculated intersection angle for this particular specimen was 1.5707 radians, which is nearly equal to the analytically derived $\pi / 2$ radians. The calculated intersection angles for the remaining specimens behaved in a very similar manner.

Figure 3.22 shows how the bisection protocol was performed on an orthotropic GLARE4-3/2-0.3 variant. Application to an orthotropic variant required no adjustments to the protocol since the observed buckling always occurred after yielding. Once again, the angle of intersection between the bisection line and the logarithmic portion is nearly equal to $\pi / 2$ radians and values of these calculated intersections for GLARE 4 are shown in Table 3.3.

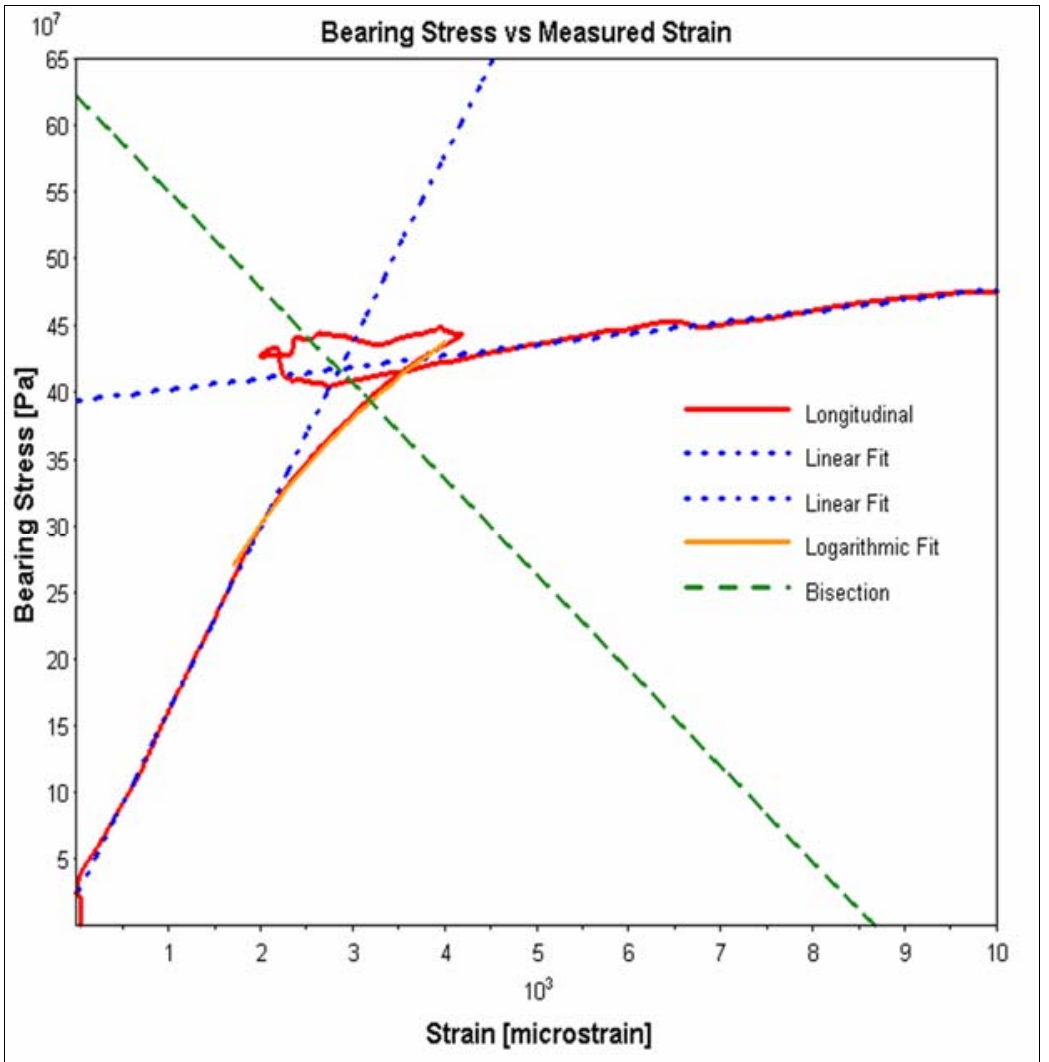

Figure 3.22: Bearing stress vs. measured strain curve for a GLARE4-3/2-0.3 specimen showing both of the linear approximations, the logarithmic approximation and the bisection line as required for the definition of bearing yield strength. 
Table 3.4 summarizes all the bearing yield strength values as per the bisection protocol for all the specimens tested and Table 3.5 shows the maximum bearing stress recorded for the GLARE4 specimens.

Table 3.3: Summary of the calculated intersection angle for GLARE4.

\begin{tabular}{cccc}
\hline GLARE Variant & $\begin{array}{c}\text { Angle of Intersection } \\
\text { [radians] }\end{array}$ & $\begin{array}{c}\text { Standard Deviation } \\
\text { [radians] }\end{array}$ & $\begin{array}{c}\text { Coefficient of } \\
\text { Variation [\%] }\end{array}$ \\
\hline GLARE4-3/2-0.3 & 1.57007 & 0.00148 & 0.09438 \\
\hline
\end{tabular}

Table 3.4: Summary of the load, strain and stress as calculated by the bisection protocol for all GLARE variants tested.

\begin{tabular}{ccccccc}
\hline $\begin{array}{c}\text { GLARE } \\
\text { Variant }\end{array}$ & $\begin{array}{c}\text { Bearing } \\
\text { Load [N] }\end{array}$ & $\begin{array}{c}\text { Standard } \\
\text { Deviation } \\
{[\mathbf{N}]}\end{array}$ & $\begin{array}{c}\text { Bearing } \\
\text { Strain }[\boldsymbol{\mu} \boldsymbol{}]\end{array}$ & $\begin{array}{c}\text { Standard } \\
\text { Deviation } \\
{[\mu \varepsilon]}\end{array}$ & $\begin{array}{c}\text { Bearing } \\
\text { Yield } \\
\text { Strength } \\
{[\mathbf{M P a}]}\end{array}$ & $\begin{array}{c}\text { Standard } \\
\text { Deviation } \\
{[\mathbf{M P a}]}\end{array}$ \\
\hline $\begin{array}{c}\text { GLARE3- } \\
\text { 5/4-0.3 }\end{array}$ & 4168.772 & 329.562 & 2991.737 & 255.210 & 339.217 & 26.189 \\
\hline $\begin{array}{c}\text { GLARE3- } \\
\text { 4/3-0.3 }\end{array}$ & 3593.504 & 517.544 & 3332.138 & 558.358 & 375.854 & 53.341 \\
\hline $\begin{array}{c}\text { GLARE4- } \\
\text { 3/2-0.3 }\end{array}$ & 3247.750 & 215.447 & 2812.828 & 469.557 & 365.676 & 24.258 \\
\hline
\end{tabular}

Table 3.5: Summary of the maximum bearing load and associated bearing stress for a GLARE4 variant.

\begin{tabular}{cccccc}
\hline $\begin{array}{c}\text { GLARE } \\
\text { Variant }\end{array}$ & $\begin{array}{c}\text { Maximum } \\
\text { Bearing } \\
\text { Load [N] }\end{array}$ & $\begin{array}{c}\text { Standard } \\
\text { Deviation [N] }\end{array}$ & $\begin{array}{c}\text { Maximum } \\
\text { Bearing } \\
\text { Stress [MPa] }\end{array}$ & $\begin{array}{c}\text { Standard } \\
\text { Deviation } \\
\text { [MPa] }\end{array}$ & $\begin{array}{c}\text { Coefficient } \\
\text { of Variation } \\
\text { [\%] }\end{array}$ \\
\hline $\begin{array}{c}\text { GLARE4- } \\
\text { 3/2-0.3 }\end{array}$ & 4226.794 & 74.174 & 475.909 & 8.532 & 1.755 \\
\hline
\end{tabular}




\subsubsection{Detection of Buckling in the Aluminum Layers}

Thus far the focus of the discussion has been on the extraction of bearing yield values as per the acquired strain profile from the longitudinally oriented strain gage.

Characterization of the material response through the acquired longitudinal signal is not the only means of extracting data from the test.

The transversely oriented gage used in the orthotropic specimens (whose response is depicted in Figure 3.23) can be used both on its own and in conjunction with the longitudinal gage to detect the occurrence of buckling throughout the prescribed testing window. The correspondence between the two curves is clearly visible, complete with the anticipated reversal in signal sense for the transverse gage. In addition, upon further examination both curves exhibit local reversals in signal sense at the same applied bearing stress, which can be attributed to a change in the specimen compliance. This is symptomatic of local buckling which involves a change in the specimen strain without a corresponding increase in the load carrying capacity. Since the compliance change was captured by both gages simultaneously, on every specimen examined, it reduces the likelihood of it being a chance occurrence and as it was previously stated, the onset of yield according to the bisection protocol always occurred just before buckling. Furthermore, if the actual bearing stress associated with the onset of buckling is extracted from all the tested specimens we see that it takes a mean value of $420.069 \mathrm{MPa}$ with a low coefficient of variation near $8.89 \%$. Thus, when compared to the calculated bearing yield strength of GLARE4 from above, we see a relative difference between the two of approximately $12.94 \%$. 


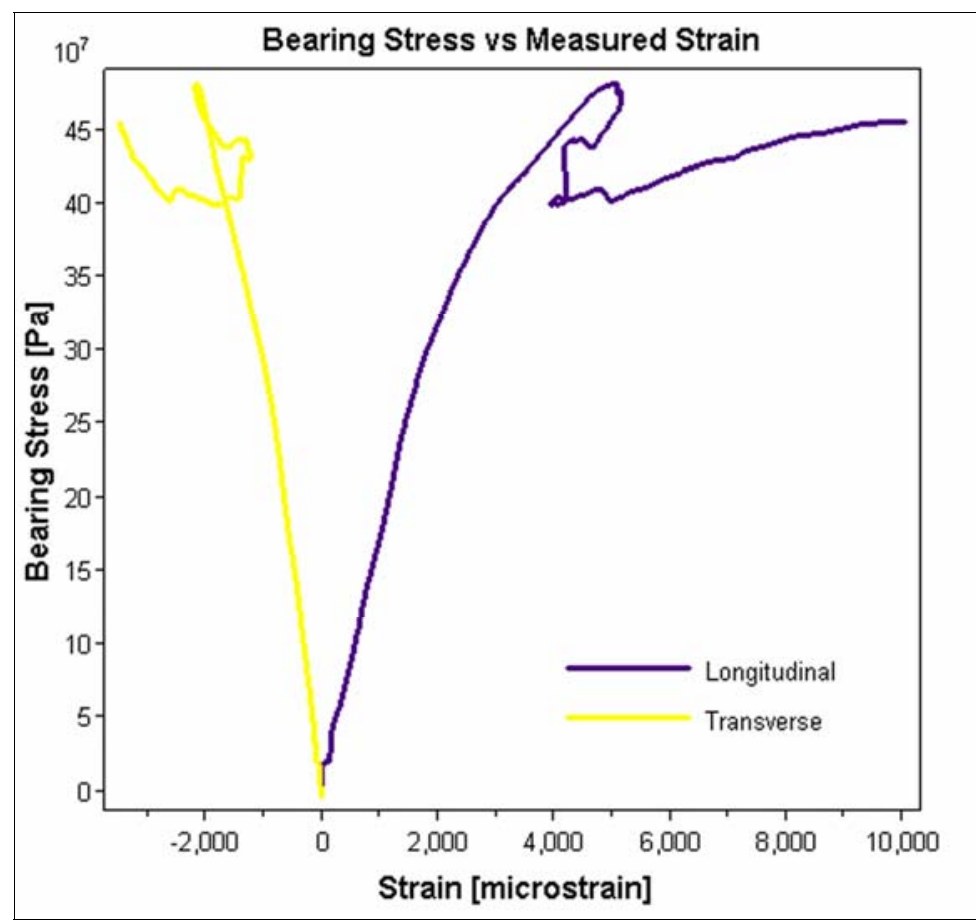

Figure 3.23: Bearing stress vs. measured strain curves for both the longitudinally and transversely oriented gages.

This is in support of the YDB mechanism which states that yielding of the aluminum would occur first, followed by a rapid delamination through the transfer of load leaving the plies laterally unsupported and prone to buckling.

\subsubsection{Analysis of Buckling in the Aluminum Layers}

An analytical approach can also serve to illustrate the occurrence of buckling for the examined load case. It was proposed and discussed earlier in Chapter 2 that, in a conservative sense, delamination has occurred in the material located within the yielded distance stipulated by the modified CCD.

Taking this one step further, a rectangular profile for a delaminated region can be generated by also assuming, again at the very least, that delamination has also occurred in 
the transverse direction in which the effective Von Mises stress has fallen beneath the yield stress of the aluminum. These two stipulated distances provide precise boundaries for a delaminated region taken directly from the FE analysis. From here, an orthotropic plate buckling analysis was performed on the laterally unsupported glass layers, where their contribution to overall pin bearing strength was found to be small and insignificant. In an entirely analogous fashion, the dimensions of a laterally unsupported layer of aluminum could also be estimated from the calculated modified CCD value and a second buckling analysis could be performed where the plate length, from the previous CCD calculations, is $3.886 \mathrm{~mm}$ and the plate width is $5.483 \mathrm{~mm}$. Precisely such an analysis was indeed conducted and it was assumed that the prescribed boundary conditions for the edges of the plate would be simply supported for the three unloaded edges and the loading edge left free to deflect. This most closely resembles the actual conditions of an outermost layer of aluminum buckling after having delaminated.

The expression for calculating the critical buckling stress $\left(\sigma_{\mathrm{cr}}\right)$ for a plate subjected to a compressive load and simply supported on three sides is given by Equation (3.6) [115]:

$$
\left(\sigma_{x}\right)_{c r}=\frac{\left(N_{x}\right)_{c r}}{t}=k \frac{\pi^{2} D}{b^{2} h} \text { where } D=\frac{E t^{3}}{12\left(1-v^{2}\right)}
$$

The parameter $k$ is a numerical factor depending on the magnitude of the aspect ratio $a / b$ and is typically plotted on charts of increasing aspect ratio. For the examined case with the prescribed boundary conditions, the corresponding value of this buckling constant was interpolated with a $4^{\text {th }}$ order polynomial through the provided plotted profile and takes on a value of 2.238 [115]. Substitution of this value in Equation (3.6), along with the material properties for 2024-T3 aluminum, produces a theoretical value of $\sigma_{\mathrm{cr}}$ equal to 
441.639 MPa. When compared against the aforementioned, empirically derived values of bearing stress at buckling onset, we see a relative error of 5.13\%. Please note that a short script detailing the polynomial fitting and calculation of critical buckling stress can be found in the appendices.

The above result is a reasonable approximation since the critical stress, calculated in this fashion, represents the true critical stress provided it is below the proportional limit of the material. Above this limit, Equation (3.6) produces slightly exaggerated value for $\sigma_{\mathrm{cr}}$ and the true value of this stress can be obtained only by incorporating the effects of plasticity [115]. This however, is a very involved process and requires a separate set of experiments to extract more material properties such as but not limited to, the tangent modulus $\mathrm{E}_{\mathrm{t}}$. The purpose and true motivation behind this analysis was the determination of a critical buckling stress such that it could provide a reasonable benchmark for comparison to the empirically derived values. Excessively high experimental results, relative to an analytically derived value, would require further discussion. That the calculated result is slightly higher than one with plasticity included is incidental since the small improvement in accuracy would serve only as a refinement after a suitable benchmark was already established in the present manner and would have little impact on the existing comparison between extracted bearing yield and the measured stress at buckling. An examination of these results and the acquired strain profiles suggests a relationship between yielding and buckling with the bearing yield occurring first and the yield strength of the aluminum representing the governing factor. These observed and calculated results support the YDB mechanism. 
Not to be forgotten, the tendency for specimen delamination is not ignored, rather, for the present investigation and scope of this section it is assumed to be present in the capacity of providing the dimensions of the laterally unsupported regions which cause both the orthotropic plate buckling and the now identified aluminum buckling. This leaves an investigation into the influence of delamination and the contribution of its onset and propagation to joint collapse open for examination. This represents an important investigation which was conducted and will be discussed alongside the progressive damage FE and delamination modeling of the next chapter.

\subsection{GLARE Tension Experiments}

The aim of this section is to further evaluate the effectiveness of the piecewise material characterization and the bisection protocol, but this time not on a pin bearing loading configuration but rather on a standard tensile test. Yield will be calculated according to standardized protocols as well as the bisection protocol and a discussion of the results of

the tests will be presented. Direct comparisons between each yield definition will also be shown and commentary regarding the statistics and behaviour of each will also be included.

\subsubsection{GLARE Tension Test Specimen Fabrication and Preparation}

Investigations into the tensile behaviour of FMLs typically employ ASTM D3039 [116] which is the standard test method for tensile properties of polymer matrix composite materials. All GLARE tension testing performed herein was in compliance to that 
standard. The only deviation from the standard is the employment of biaxial strain gages rather than two single axis gages bonded perpendicularly as well as the additional calculation of yield according to the bisection protocol. The purpose of applying a second, transversely oriented gage is for the measurement of induced transverse strain for the calculation of Poisson's ratio.

Both quasi-isotropic and fully orthotropic variants were investigated, namely the GLARE3-5/4-0.3, GLARE3-4/3-0.3 and GLARE4-3/2-0.3 variants, respectively. Figure 3.24 shows a typical tension specimen with a length of $304.8 \mathrm{~mm}$ and a width of $50.4 \mathrm{~mm}$ as per the standard. Specimens of each variant differed only by their thickness. Geometric tolerances were tightly adhered to for the assurance of fibre direction and rolling

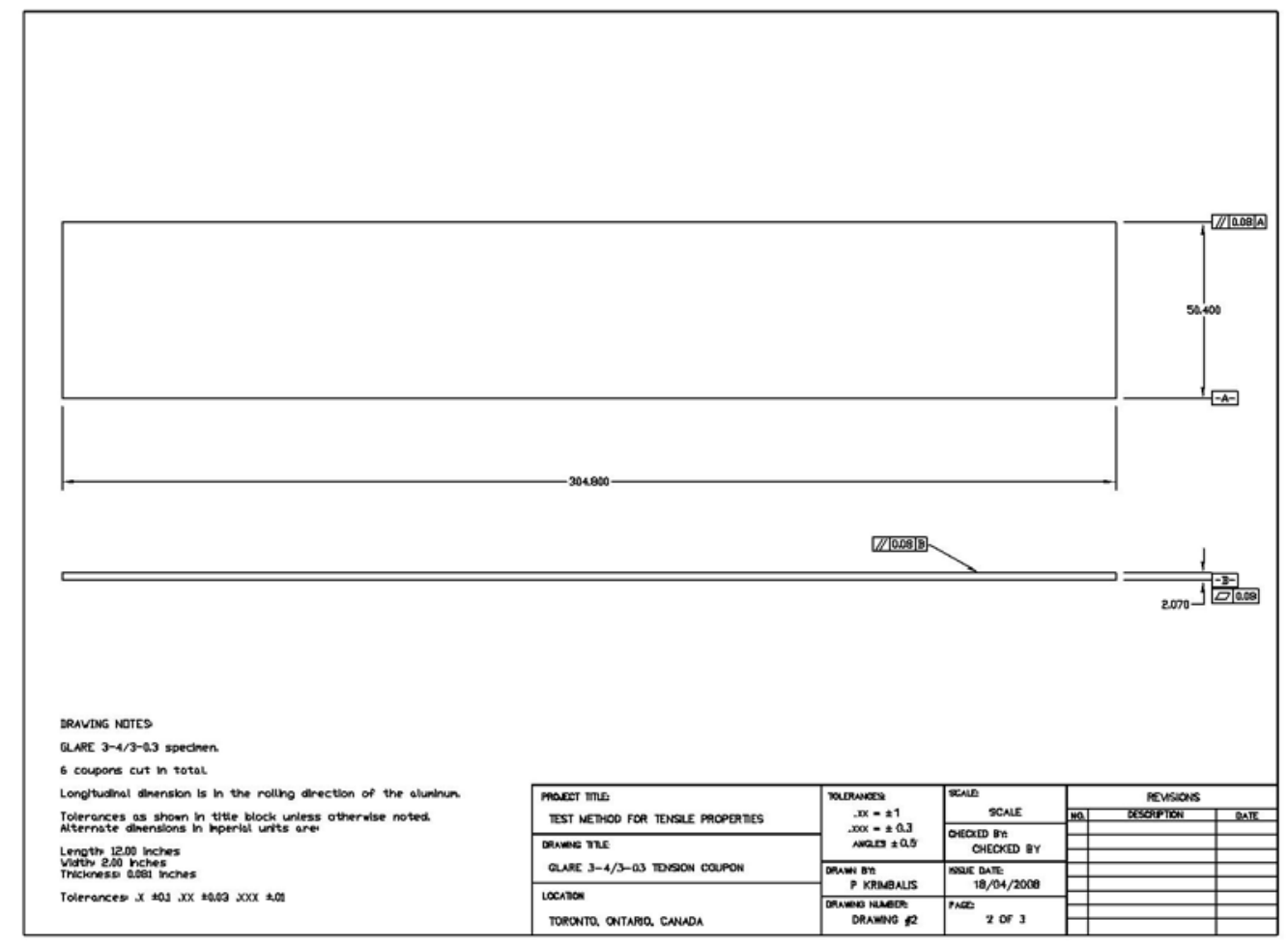

Figure 3.24: Dimensions of a typical GLARE FML tension testing specimen. 
direction of the aluminum remain in line with the applied loading. Please note, as per the recommendations of the standard, the employment of the above dimensions for the specimens negated the need for bonded end tabs, greatly simplifying the fabrication process.

The strain gages used were $120 \Omega$, Omega SGD-7/120-XY43 Precision Strain gages supplied by Omega (Omega Inc., Laval, Quebec, Canada) and were temperature compensated for adhesion to an aluminum substrate. Figure 3.25 depicts a typical tension test specimen with the biaxial strain gage already applied and ready for testing. In addition to the biaxial gage applied to the front of the specimen, one specimen from each variant analyzed also included a second gage bonded in the same central location but now on the reverse side of the specimen for the measurement of out of plane bending. Table 3.6 summarizes the all of the specimens tested and the number of gages applied.

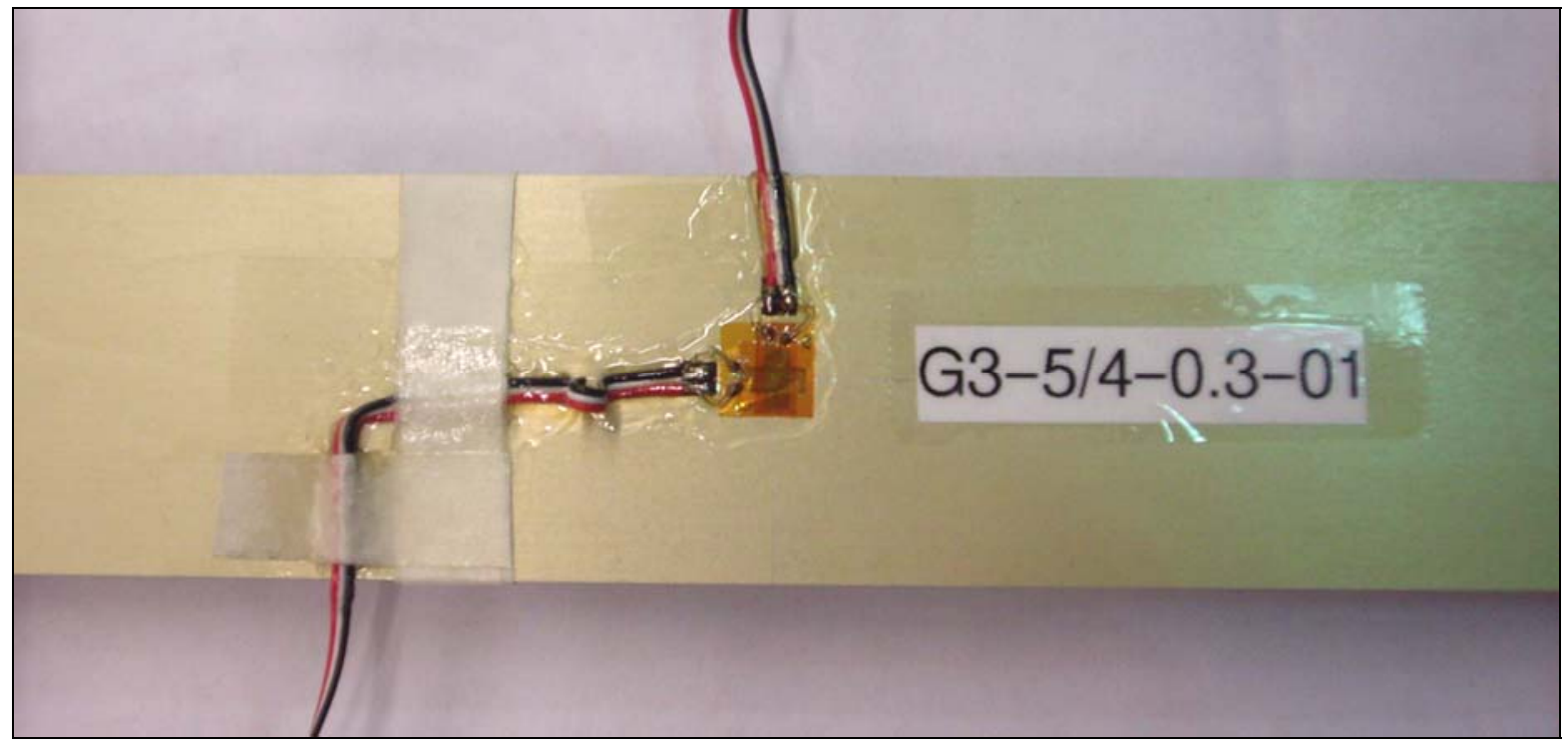

Figure 3.25: Typical GLARE tension test specimen with biaxial strain gage applied. 
Table 3.6: Summary of the test matrix employed. Note: btb indicates back to back gages were applied.

\begin{tabular}{ccc}
\hline GLARE Variant & Number of Specimens & Biaxial Gages Applied \\
\hline GLARE3-5/4-0.3 & 5 & $\frac{\text { Single gage: } 4 \text { specimens }}{\text { Two gages } b t b .: \text { 1 specimen }}$ \\
\hline GLARE3-4/3-0.3 & 5 & $\frac{\text { Single gage: } 4 \text { specimens }}{\text { Two gages } b t b .: \text { 1 specimen }}$ \\
\hline GLARE4-3/2-0.3 & 5 & Single gage: 4 specimens \\
\hline
\end{tabular}

\subsubsection{Experimental Protocol for the GLARE Tension Tests}

All experiments were carried out under the same conditions and with the same equipment as the pin bearing experiments. Actuator displacement was allowed to continue until complete failure of the test specimen. In addition to the applied bonded strain gages an MTS 634.12F-24 calibrated extensometer was also employed as shown in Figure 3.26. Though the standard did not stipulate the use of both an extensometer and bonded strain gages, for the sake of completeness, both were utilized as a measure of deformation and a comparison of their results will be presented.

\subsection{Results and Discussion of the GLARE Tension Tests}

In the following sections, the results from the tension test experiments performed on GLARE3-5/4-0.3, GLARE3-4/3-0.3 and GLARE4-3/2-0.3 will be presented. A presentation of the characterization of the material response and extraction of yield strength according to conventional definitions and the bisection protocol is also included. 


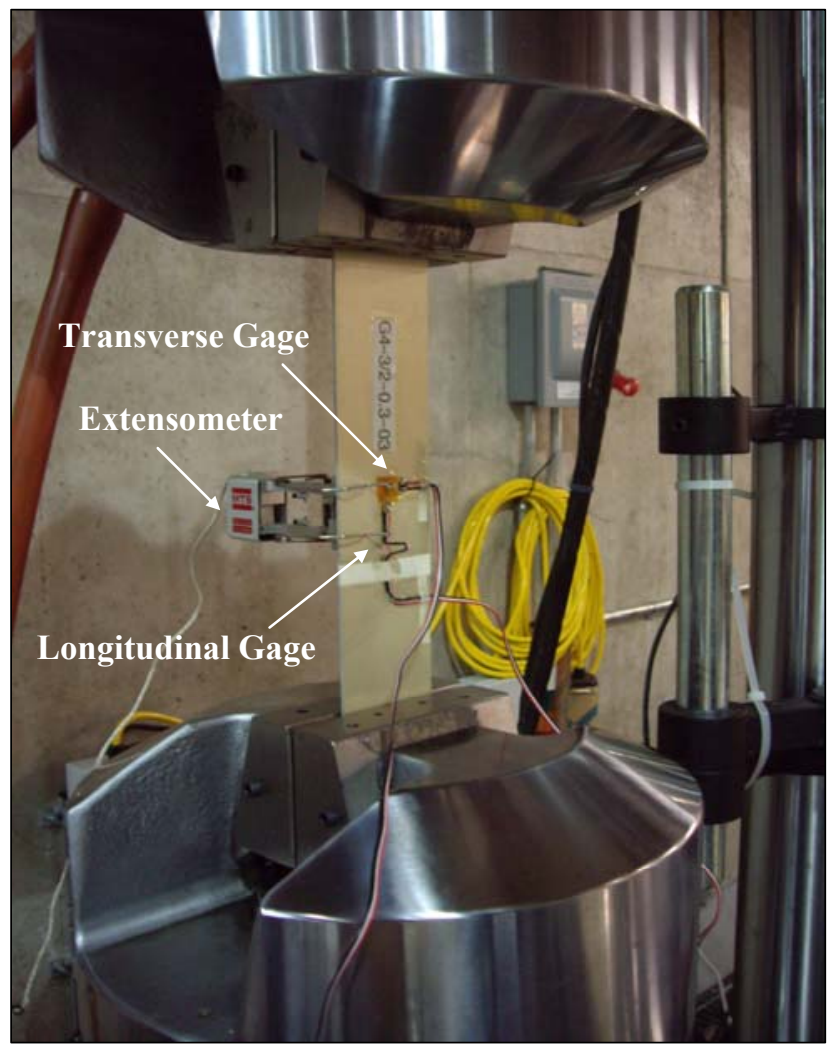

Figure 3.26: MTS 322 load frame with GLARE specimen mounted and extensometer/strain gage in place for the measurement of specimen deformation.

Data acquired from the tested specimens was imported into and analyzed by Maple V10 software [114] using custom written routines. Raw data was filtered using a 15 point moving average algorithm to smooth out local fluctuations in signal.

Figure 3.27 through Figure 3.29 depict tensile stress versus strain curves for all the specimens tested across all the variants considered. These curves were produced using the strain data recorded from the bonded strain gages. Theses curves closely resemble the generated curves from the pin bearing experimentation making the application of the bisection protocol highly attractive. Furthermore, Figure 3.30 depicts a single GLARE34/3-0.3 specimen's tensile stress versus strain curve as measured by both the extensometer and longitudinal strain gage. Note that the acquired signals are nearly equal 


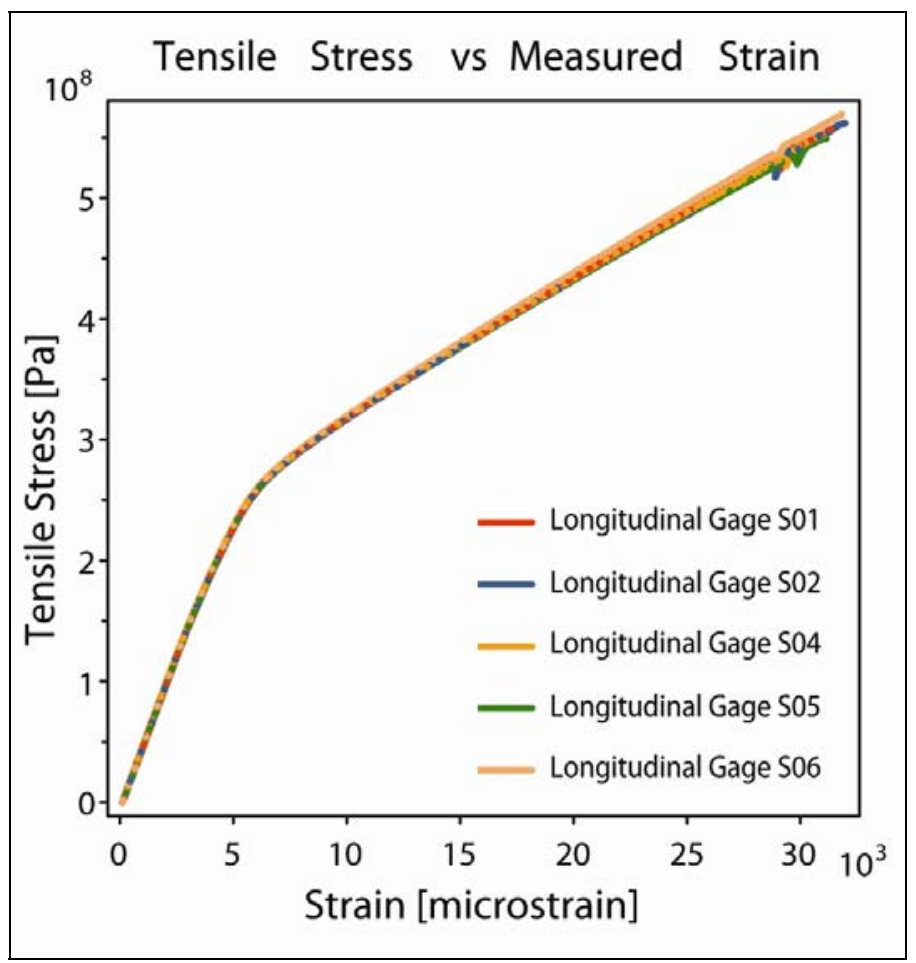

Figure 3.27: Tensile stress versus strain as measured by the bonded strain gage for all tested specimens of a GLARE3-4/3-0.3 variant.

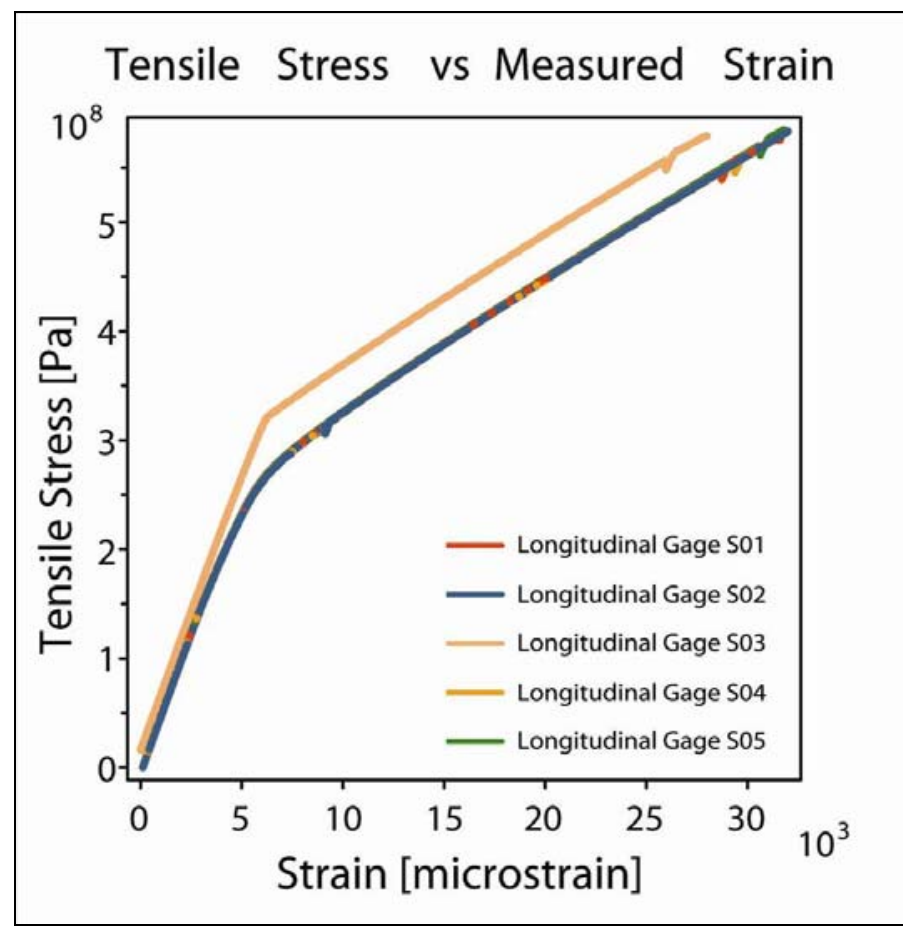

Figure 3.28: Tensile stress versus strain as measured by the bonded strain gage for all tested specimens of a GLARE3-5/4-0.3 variant. 


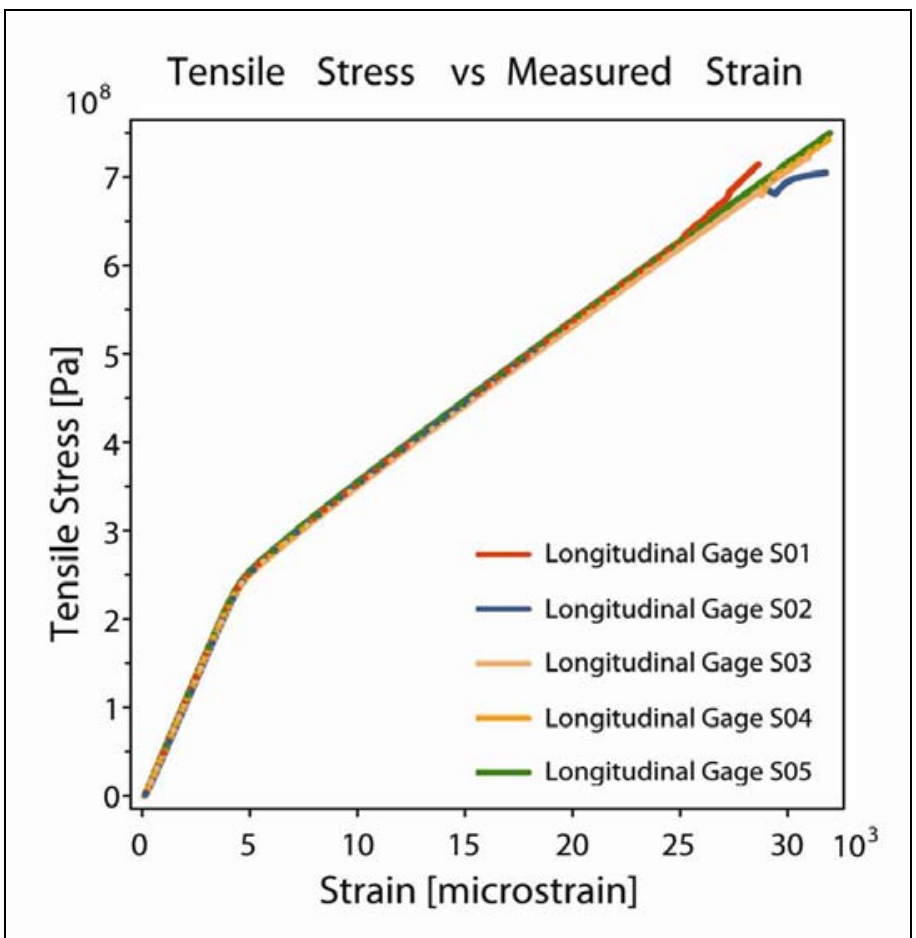

Figure 3.29: Tensile stress versus strain as measured by the bonded strain gage for all tested specimens of a GLARE4-3/2-0.3 variant.

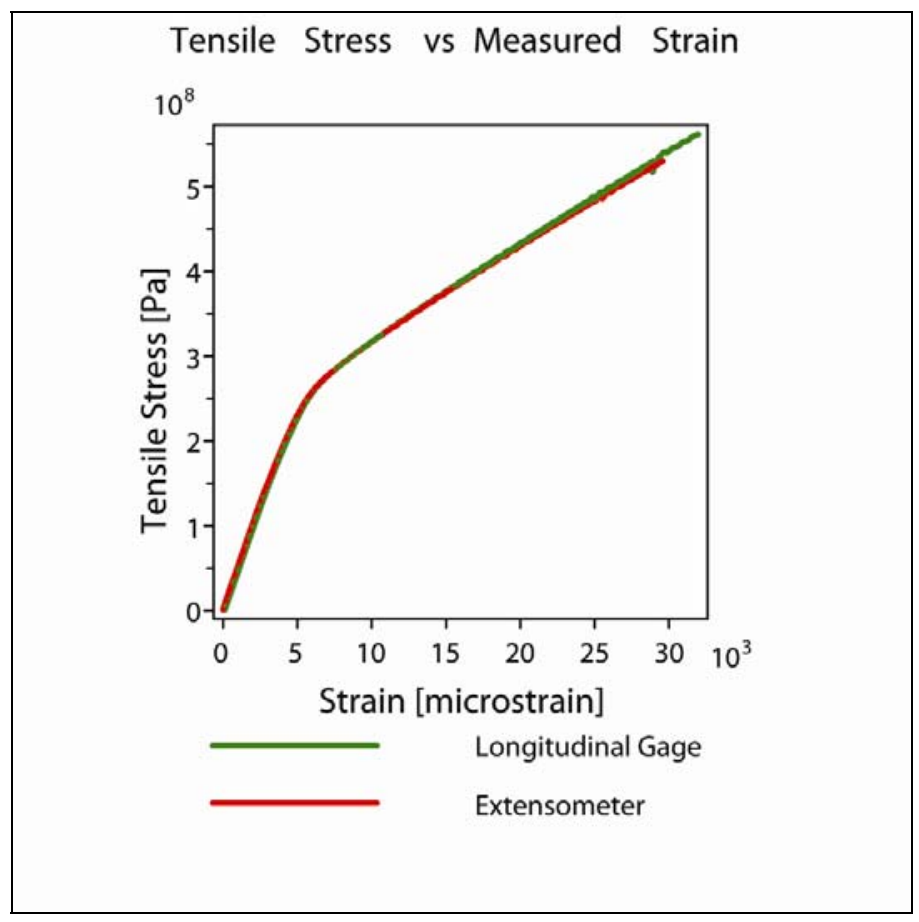

Figure 3.30: Direct comparison of material behaviour as measure by the extensometer and longitudinal gage for a GLARE3-4/3-0.3 variant. 
to each other as was the case for all the specimens tested. Therefore it can be safely said that an accurate and consistent measurement of the mechanical response has been captured.

With an accurate measure of specimen response, the material's profile was then characterized employing the same piecewise approximation as before as shown in Figure 3.31, without any adjustments necessary to the protocol.

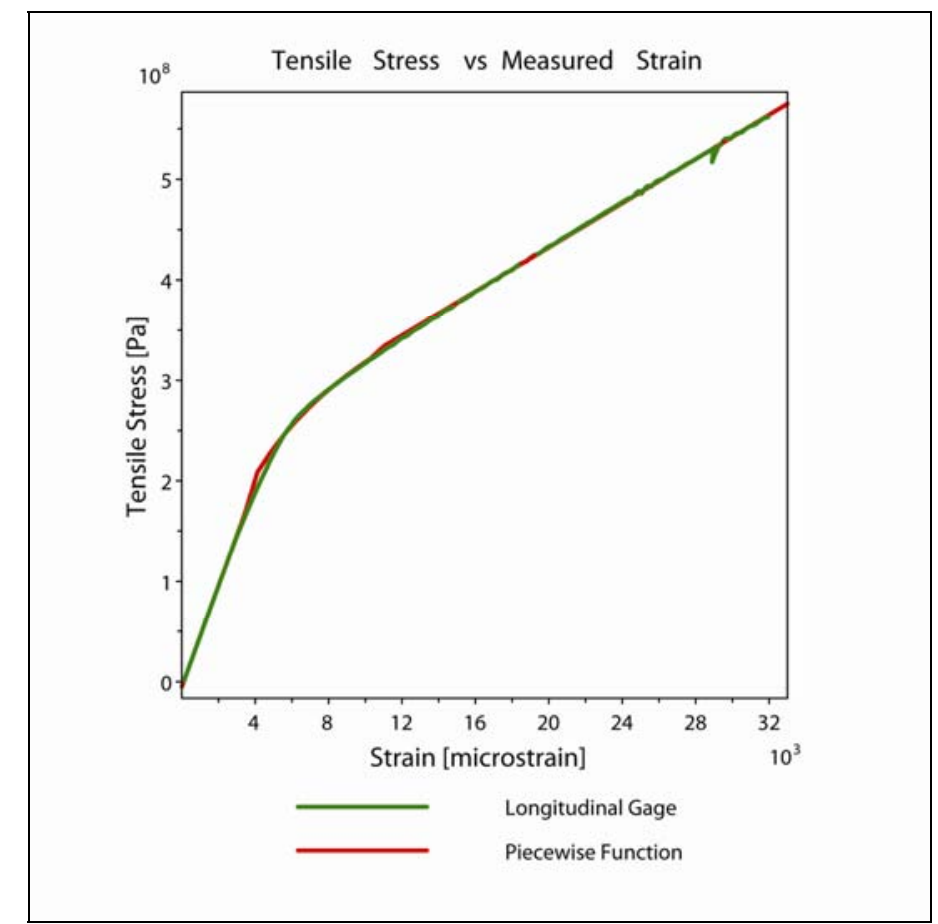

Figure 3.31: Piecewise characterization of the tensile stress versus strain profile of a typical GLARE variant.

\subsubsection{Calculation of Standardized Parameters}

Numerous material parameters such as the tensile chord modulus, the ultimate tensile stress and Poisson's ratio were required calculations as per the standard. Table 3.7 through Table 3.9 summarize these calculations along with their associated statistics. 
Table 3.7: Calculated ultimate tensile stress and its associated statistics for all GLARE variants tested.

\begin{tabular}{cccc}
\hline GLARE Variant & $\begin{array}{c}\text { Average Ultimate } \\
\text { Tensile Stress [MPa] }\end{array}$ & $\begin{array}{c}\text { Standard } \\
\text { Deviation [MPa] }\end{array}$ & $\begin{array}{c}\text { Coefficient of } \\
\text { Variation [\%] }\end{array}$ \\
\hline GLARE3-5/4-0.3 & 645.009 & 24.661 & 3.82 \\
\hline GLARE3-4/3-0.3 & 586.040 & 26.249 & 4.47 \\
\hline GLARE4-3/2-0.3 & 773.279 & 25.508 & 3.29 \\
\hline
\end{tabular}

Table 3.8: Calculated tensile chord modulus and associated statistics as measured by the longitudinal strain gage and the extensometer.

\begin{tabular}{ccccccc}
\hline & \multicolumn{3}{c}{ Strain Gage Measurement } & \multicolumn{2}{c}{ Extensometer Measurement } \\
\cline { 2 - 6 } GLARE \\
\cline { 2 - 6 } Variant & $\begin{array}{c}\text { Tensile } \\
\text { Chord } \\
\text { Modulus } \\
\text { [GPa] }\end{array}$ & $\begin{array}{c}\text { Standard } \\
\text { Deviation } \\
{[\text { [GPa] }}\end{array}$ & $\begin{array}{c}\text { Coefficient } \\
\text { of } \\
\text { Variation } \\
{[\%]}\end{array}$ & $\begin{array}{c}\text { Tensile } \\
\text { Chord } \\
\text { Modulus } \\
\text { [GPa] }\end{array}$ & $\begin{array}{c}\text { Standard } \\
\text { Deviation } \\
{[\text { [GPa] }}\end{array}$ & $\begin{array}{c}\text { Coefficient } \\
\text { of } \\
\text { Variation } \\
{[\%]}\end{array}$ \\
\hline $\begin{array}{c}\text { GLARE3- } \\
\text { 5/4-0.3 }\end{array}$ & 50.523 & 0.118 & 0.23 & 50.771 & 1.294 & 2.54 \\
\hline $\begin{array}{c}\text { GLARE3- } \\
\text { 4/3-0.3 }\end{array}$ & 50.053 & 0.398 & 0.79 & 49.672 & 0.563 & 1.13 \\
\hline GLARE4- & 56.079 & 0.123 & 0.22 & 55.173 & 0.705 & 1.27 \\
\hline 3/2-0.3 & & & & & \\
\hline
\end{tabular}

Table 3.9: Calculated Poisson's ratio for all GLARE variants tested.

\begin{tabular}{cccc}
\hline GLARE Variant & $\begin{array}{c}\text { Average Poisson's } \\
\text { Ratio [/] }\end{array}$ & $\begin{array}{c}\text { Standard } \\
\text { Deviation [/] }\end{array}$ & $\begin{array}{c}\text { Coefficient of } \\
\text { Variation [\%] }\end{array}$ \\
\hline GLARE3-5/4-0.3 & 0.277 & 0.0029 & 1.05 \\
\hline GLARE3-4/3-0.3 & 0.282 & 0.0023 & 0.84 \\
\hline GLARE4-3/2-0.3 & 0.292 & 0.0027 & 0.92 \\
\hline
\end{tabular}


In addition to the standard calculations, the standard also required the calculation of the percent bending of the system. This out of plane bending occurs as a result of misalignment between the hydraulic grips of the employed universal testing machine. Any bending out of plane would manifest as a difference between the gage on the front and the gage on the back, which were applied to one specimen of each variant. The calculation of out of plane bending is presented as a percentage and is accomplished via Equation (3.7) where $\varepsilon_{\mathrm{f}}$ is the strain recorded by the front gage and $\varepsilon_{\mathrm{b}}$ is the strain recorded by the back gage [116]:

$$
B_{y}=\frac{\left|\varepsilon_{f}-\varepsilon_{b}\right|}{\left|\varepsilon_{f}+\varepsilon_{b}\right|}
$$

The standard stipulates that a percent out of plane bending less than $3 \%$ is considered acceptable and thus the testing can proceed with front bonded gages only [116]. Table 3.10 summarizes the calculated percent out of plane bending for all the variants tested. Clearly the calculated percent bending is much smaller than the stipulated $3 \%$ and thus the remaining specimens only had gages on the front.

Table 3.10: Calculated percent bending as measured by the back to back gages for all variants tested.

\begin{tabular}{cc}
\hline GLARE Variant & Percent Bending [\%] \\
\hline GLARE3-5/4-0.3 & 0.08 \\
\hline GLARE3-4/3-0.3 & 0.008 \\
\hline GLARE4-3/2-0.3 & 0.007 \\
\hline
\end{tabular}




\subsubsection{Calculation of Yield Strength}

With the above standardized parameters calculated according to ASTM D 3039 [116], the only remaining parameter - chiefly the one of interest to this portion of the study - is that of tensile yield strength. That being said, the bisection protocol outlined in the pin bearing experiments was employed without adjustment to the tensile load case. Both the previously shown piecewise characterization and the bisection protocol were applied and subsequently evaluated. Figure 3.32 depicts a typical GLARE specimen with the bisection yield protocol and Table 3.11 summarizes the averaged results and the associated statistics from all the tensile stress strain profiles as measured by both the longitudinal gage and the extensometer. Table 3.12 summarizes the calculated intersection angle as required by the bisection protocol.

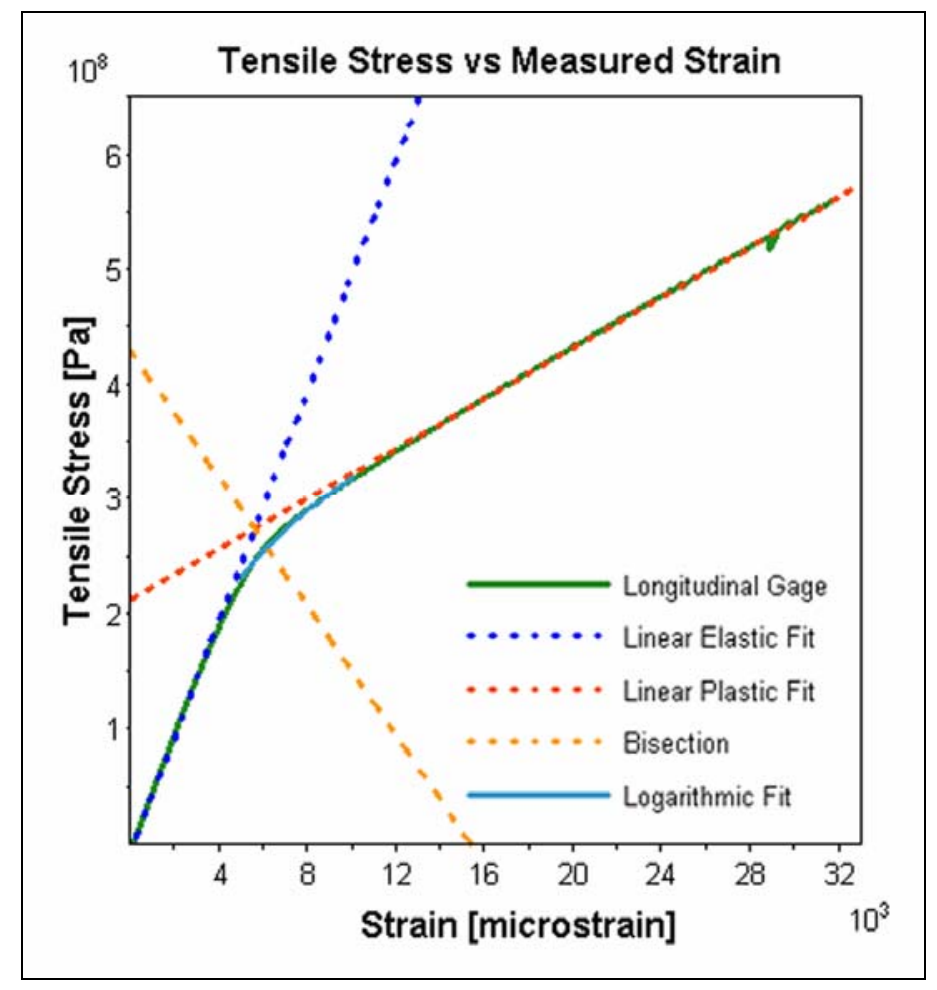

Figure 3.32: Bisection protocol yield definition as applied to a typical GLARE variant tensile specimen. 
Table 3.11: Summary of the calculated tensile yield strength as measured by the longitudinal strain gage and the extensometer according to the bisection protocol.

\begin{tabular}{ccccccc}
\hline & \multicolumn{2}{c}{ Strain Gage Measurement } & \multicolumn{2}{c}{ Extensometer Measurement } \\
\cline { 2 - 7 } $\begin{array}{c}\text { GLARE } \\
\text { Variant }\end{array}$ & $\begin{array}{c}\text { Tensile } \\
\text { Yield } \\
\text { Strength } \\
{[\text { [MPa] }}\end{array}$ & $\begin{array}{c}\text { Standard } \\
\text { Deviation } \\
{[\text { [MPa] }}\end{array}$ & $\begin{array}{c}\text { Coefficient } \\
\text { of } \\
\text { Variation } \\
{[\%]}\end{array}$ & $\begin{array}{c}\text { Tensile } \\
\text { Yield } \\
\text { Strength } \\
{[M P a]}\end{array}$ & $\begin{array}{c}\text { Standard } \\
\text { Deviation } \\
{[M P a]}\end{array}$ & $\begin{array}{c}\text { Coefficient } \\
\text { of } \\
\text { Variation } \\
{[\%]}\end{array}$ \\
\hline $\begin{array}{c}\text { GLARE3- } \\
\text { 5/4-0.3 }\end{array}$ & 274.585 & 23.914 & 8.70 & 278.274 & 26.553 & 9.54 \\
\hline $\begin{array}{c}\text { GLARE3- } \\
\text { 4/3-0.3 }\end{array}$ & 258.684 & 0.865 & 0.33 & 258.168 & 0.772 & 0.29 \\
\hline $\begin{array}{c}\text { GLARE4- } \\
\text { 3/2-0.3 }\end{array}$ & 251.103 & 8.371 & 3.33 & 249.907 & 1.842 & 0.73 \\
\hline
\end{tabular}

Table 3.12: Calculated intersection angle as defined by the bisection protocol.

\begin{tabular}{ccccccc}
\hline & \multicolumn{2}{c}{ Strain Gage Measurement } & \multicolumn{2}{c}{ Extensometer Measurement } \\
\cline { 2 - 7 } $\begin{array}{c}\text { GLARE } \\
\text { Variant }\end{array}$ & $\begin{array}{c}\text { Bisection } \\
\text { Angle } \\
\text { [Radians] }\end{array}$ & $\begin{array}{c}\text { Standard } \\
\text { Deviation } \\
\text { [Radians] }\end{array}$ & $\begin{array}{c}\text { Coefficient } \\
\text { of } \\
\text { Variation } \\
\text { [\%] }\end{array}$ & $\begin{array}{c}\text { Bisection } \\
\text { Angle } \\
\text { [Radians] }\end{array}$ & $\begin{array}{c}\text { Standard } \\
\text { Deviation } \\
\text { [Radians] }\end{array}$ & $\begin{array}{c}\text { Coefficient } \\
\text { of } \\
\text { Variation } \\
{[\%]}\end{array}$ \\
\hline $\begin{array}{c}\text { GLARE3- } \\
\text { 5/4-0.3 }\end{array}$ & 1.57076 & $1.1 \times 10^{-7}$ & $7.0 \times 10^{-6}$ & 1.57076 & $2.7 \times 10^{-7}$ & $1.7 \times 10^{-5}$ \\
\hline $\begin{array}{c}\text { GLARE3- } \\
\text { 4/3-0.3 }\end{array}$ & 1.57076 & $6.6 \times 10^{-7}$ & $4.2 \times 10^{-5}$ & 1.57075 & $5.4 \times 10^{-7}$ & $3.4 \times 10^{-5}$ \\
\hline $\begin{array}{c}\text { GLARE4- } \\
\text { 3/2-0.3 }\end{array}$ & 1.57077 & $4.4 \times 10^{-7}$ & $2.8 \times 10^{-5}$ & 1.57077 & $3.7 \times 10^{-7}$ & $2.4 \times 10^{-5}$ \\
\hline
\end{tabular}

What is important about the values shown in both tables is first the consistency in the calculated values between those generated from strain gage data and those generated from the extensometer data. Secondly, the calculated bisection angle is once again, as shown in the pin bearing experimental results, nearly equal to the predicted value of $\pi / 2$ radians. 
The associated error between the calculated values and that of $\pi / 2$ is limited to the fifth decimal place making it by far the most accurate prediction shown thus far in the study. With the tensile yield strengths calculated according to the bisection protocol, additional definitions of yield were also calculated as a comparison. These definitions included the previously mentioned bilinear idealization and the $0.2 \%$ offset technique. Figure 3.32 and Figure 3.33 and Figure 3.34 show the bilinear idealization and the $0.2 \%$ offset respectively, as performed on two typical specimens, the first being a GLARE3-4/3-0.3 variant and the second a GLARE4-3/2-0.3 variant.

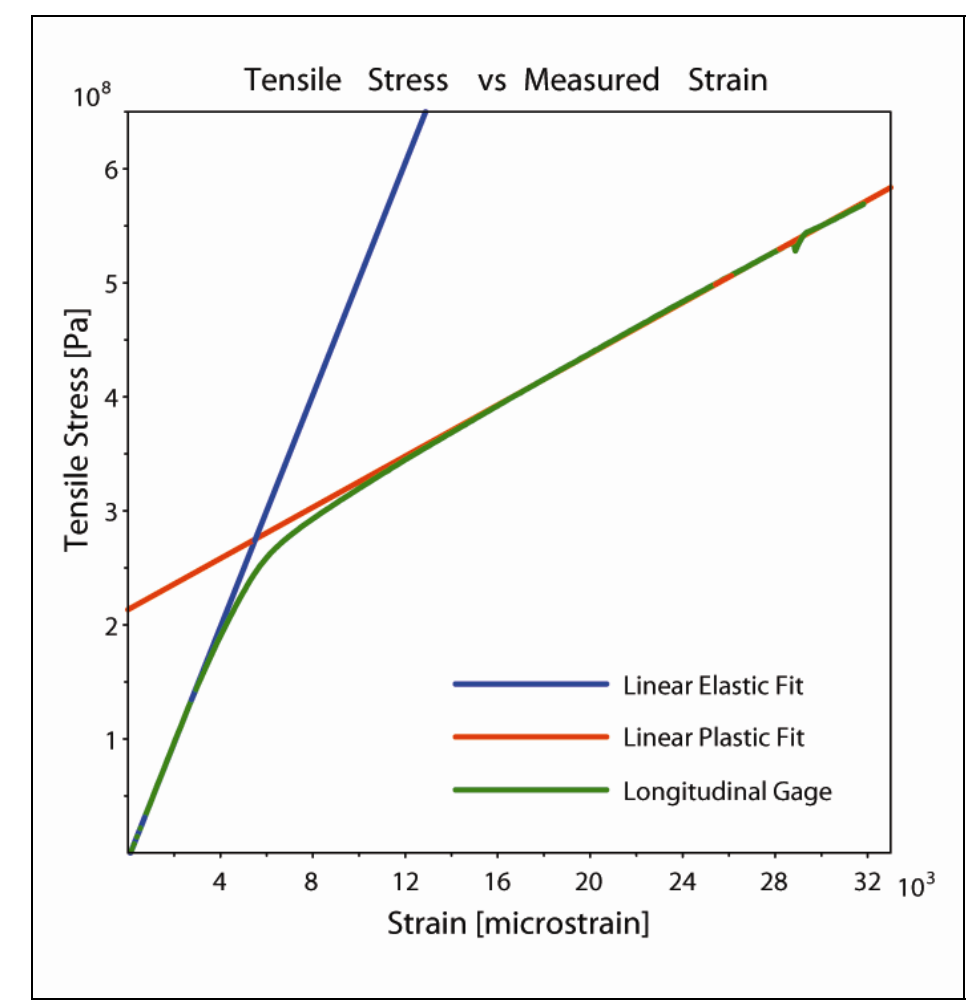

Figure 3.33: Bilinear idealization as performed on a GLARE3-4/3-0.3 variant. 


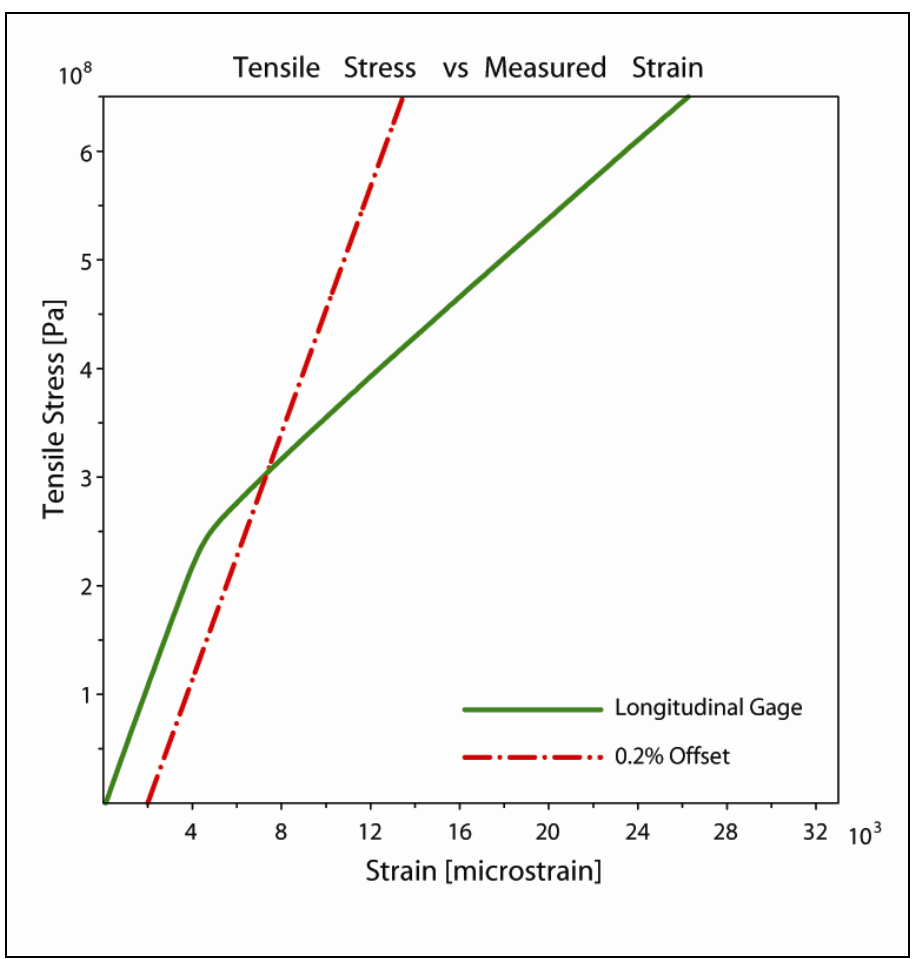

Figure 3.34: The $0.2 \%$ offset technique as performed on a GLARE4-3/2-0.3 variant.

Upon inspection of Figure 3.33 we see that the bilinear tensile yield strength - stated as the point of intersection between the two linear approximations - does not lie upon the acquired curve and overestimates the yield strength of the material. Figure 3.34 shows that while the offset technique produces a value that does in fact lie on the curve, the value it produces is rather high and overshoots the non-linear region entirely.

Table 3.13 summarizes the results of the bilinear idealization,

Table 3.14 summarizes the results of the offset protocol and Table 3.15 shows their relatives errors to each other. 
In general, the bisection protocol produces results with the smallest amount of statistical scatter suggesting a stabilizing effect due to influence from multiple regimes within the acquired curves.

Table 3.13: Summary of the calculated tensile yield strength as measured by the longitudinal strain gage and the extensometer according to the bilinear idealization.

\begin{tabular}{ccccccc}
\hline & \multicolumn{2}{c}{ Strain Gage Measurement } & \multicolumn{3}{c}{ Extensometer Measurement } \\
\cline { 2 - 7 } $\begin{array}{c}\text { GLARE } \\
\text { Variant }\end{array}$ & $\begin{array}{c}\text { Tensile } \\
\text { Yield } \\
\text { Strength } \\
{[\text { [MPa] }}\end{array}$ & $\begin{array}{c}\text { Standard } \\
\text { Deviation } \\
{[\text { [MPa] }}\end{array}$ & $\begin{array}{c}\text { Coefficient } \\
\text { of } \\
\text { Variation } \\
{[\%]}\end{array}$ & $\begin{array}{c}\text { Tensile } \\
\text { Yield } \\
\text { Strength } \\
{[M P a]}\end{array}$ & $\begin{array}{c}\text { Standard } \\
\text { Deviation } \\
{[M P a]}\end{array}$ & $\begin{array}{c}\text { Coefficient } \\
\text { of } \\
\text { Variation } \\
{[\%]}\end{array}$ \\
\hline $\begin{array}{c}\text { GLARE3- } \\
\text { 5/4-0.3 }\end{array}$ & 290.388 & 21.377 & 7.36 & 293.669 & 23.628 & 8.04 \\
\hline $\begin{array}{c}\text { GLARE3- } \\
\text { 4/3-0.3 }\end{array}$ & 275.089 & 1.096 & 0.39 & 274.413 & 0.798 & 0.29 \\
\hline GLARE4- & 264.999 & 11.13 & 4.20 & 265.344 & 1.909 & 0.72 \\
\hline 3/2-0.3 & & & & & \\
\hline
\end{tabular}

Table 3.14: Summary of the calculated tensile yield strength as measured by the longitudinal strain gage and the extensometer according to the $0.2 \%$ offset.

\begin{tabular}{ccccccc}
\hline & \multicolumn{2}{c}{ Strain Gage Measurement } & \multicolumn{2}{c}{ Extensometer Measurement } \\
\cline { 2 - 7 } $\begin{array}{c}\text { GLARE } \\
\text { Variant }\end{array}$ & $\begin{array}{c}\text { Tensile } \\
\text { Yield } \\
\text { Strength } \\
\text { [MPa] }\end{array}$ & $\begin{array}{c}\text { Standard } \\
\text { Deviation } \\
{[\text { [MPa] }}\end{array}$ & $\begin{array}{c}\text { Coefficient } \\
\text { of } \\
\text { Variation } \\
{[\%]}\end{array}$ & $\begin{array}{c}\text { Tensile } \\
\text { Yield } \\
\text { Strength } \\
{[M P a]}\end{array}$ & $\begin{array}{c}\text { Standard } \\
\text { Deviation } \\
{[M P a]}\end{array}$ & $\begin{array}{c}\text { Coefficient } \\
\text { of } \\
\text { Variation } \\
{[\%]}\end{array}$ \\
\hline $\begin{array}{c}\text { GLARE3- } \\
\text { 5/4-0.3 }\end{array}$ & 306.694 & 30.081 & 9.81 & 311.021 & 31.668 & 10.18 \\
\hline $\begin{array}{c}\text { GLARE3- } \\
\text { 4/3-0.3 }\end{array}$ & 286.135 & 0.653 & 0.23 & 287.725 & 1.123 & 0.39 \\
\hline $\begin{array}{c}\text { GLARE4- } \\
\text { 3/2-0.3 }\end{array}$ & 305.455 & 1.657 & 0.54 & 306.666 & 2.331 & 0.76 \\
\hline
\end{tabular}


Table 3.15: Relative error in calculated tensile yield strength between the bisection protocol, the idealized bilinear approach and the $0.2 \%$ offset technique.

\begin{tabular}{ccccccc}
\hline & \multicolumn{3}{c}{ Strain Gage Measurement } & \multicolumn{3}{c}{ Extensometer Measurement } \\
\cline { 2 - 7 } $\begin{array}{c}\text { GLARE } \\
\text { Variant }\end{array}$ & $\begin{array}{c}\text { Tensile } \\
\text { Yield } \\
\text { Strength } \\
\text { [MPa] }\end{array}$ & $\begin{array}{c}\text { Rel. Error } \\
\text { from } \\
\text { Bilinear } \\
\text { [\%] }\end{array}$ & $\begin{array}{c}\text { Rel. Error } \\
\text { from } \\
\text { Offset [\%] }\end{array}$ & $\begin{array}{c}\text { Tensile } \\
\text { Yield } \\
\text { Strength } \\
\text { [MPa] }\end{array}$ & $\begin{array}{c}\text { Rel. Error } \\
\text { from } \\
\text { Bilinear } \\
\text { [\%] }\end{array}$ & $\begin{array}{c}\text { Rel. Error } \\
\text { from Offset } \\
\text { [\%] }\end{array}$ \\
\hline $\begin{array}{c}\text { GLARE3- } \\
\text { 5/4-0.3 }\end{array}$ & 274.585 & -5.51 & -10.40 & 278.274 & -5.32 & -10.48 \\
\hline $\begin{array}{c}\text { GLARE3- } \\
\text { 4/3-0.3 }\end{array}$ & 258.684 & -5.96 & -9.59 & 258.168 & -5.91 & -10.27 \\
\hline $\begin{array}{c}\text { GLARE4- } \\
\text { 3/2-0.3 }\end{array}$ & 251.103 & -5.21 & -17.79 & 249.907 & -5.81 & -18.51 \\
\hline
\end{tabular}

Additionally, the results presented in the above tables indicate that the bisection protocol yields slightly more conservative calculations of tensile yield strength relative to the other techniques.

There is a notable increase in the relative error between the bisection protocol and the retained $0.2 \%$ offset technique when examining the results for GLARE4-3/2-0.3; or to put it in more general terms when examining the most anisotropic of the variants. This result was anticipated since it is well known that the $0.2 \%$ offset technique is applied commonly to metals with their isotropic constitutive behaviour. What this suggests is that when a technique designed for isotropic materials is applied to anisotropic ones, additional factors in the material behaviour are influential and lead to a larger variation in the results. The proposed bisection protocol, which was designed with anisotropic materials in mind, has influence from all three portions of the curve and produces values of yield that account for anisotropy. 


\subsection{Chapter Summary}

In this chapter, an experimental protocol extended from ASTM D953 [68] was developed in order to examine the local yielding of aluminum at the modified CCD. This methodology was applied to both quasi-isotropic and orthotropic specimens. A piecewise characterization of material response was proposed and a new definition of yield was applied to the stress-strain curves. Buckling in the pin bearing specimens was detected through the use of biaxial gages and suggested a relationship to the yielding of the aluminum layers. This experimental investigation supports the proposed YDB mechanism. Finally, tensile tests were performed on GLARE FMLs in order to compare the bisection protocol definition of yield to existing ones. The bisection protocol shown in this chapter applied equally as well for both quasi-isotropic and anisotropic specimens, in both the pin bearing and tensile cases. The results of this experimental investigation will also be compared to the progressive damage FE model as part of the practical methodology to be discussed in the next chapter. 


\section{Chapter Four: Progressive Damage FE Modeling of GLARE FMLs in Pin Bearing}

The presented review of the literature showed that, at the time of this writing, no practical modeling methodology for simulating pin bearing in FMLs exists which includes all critical damage modes for delamination, prepreg failure and aluminum plasticity, as well as the thermally induced residual stresses. In order to further investigate the modified CCD and YDB mechanism, precisely such a model was created; one that can be compared to the empirical results and in turn, can be used for additional predictive measures as part of a practical design methodology. A methodology such as this could greatly alleviate the required amount of testing in future applications of GLARE and FMLs in general. Analogous to the experimental investigations, models for GLARE35/4-0.3, GLARE3-4/3-0.3 and GLARE4-3/2-0.3 were created using the Python Scripting Interface available in ABAQUS [112], via the Python Programming Language [117]. Python is a dynamic, object oriented language allowing for the complete and systematic generation of all the components required for modeling and simulation without the need of using the graphical user interface (GUI) of ABAQUS. 


\subsection{Model Geometry}

The geometry modeled is similar to the CCD FE models discussed in Chapter 2 and have the same dimensions as the experimentally tested specimens. Emphasis was placed once again on using $\mathrm{E} / \mathrm{D}=3$ since it represents the critical ratio for the bearing failure mode. Layer by layer modeling was employed again, though in this instance, additional layers representing the resin rich interfaces between adjacent layers of aluminum and prepreg were modeled with a nominal thickness of $0.001 \mathrm{~mm}$.

Dimensions for the individual layers within the laminate and the lay-ups employed for each GLARE variant analyzed in the study are summarized in Table 4.1.

Table 4.1: A summary of the GLARE variants analyzed and their respective dimensions/lay-ups.

\begin{tabular}{ccccccc}
\hline $\begin{array}{c}\text { GLARE } \\
\text { Variant }\end{array}$ & $\begin{array}{c}\text { Prepreg } \\
\text { Lay-up } \\
\text { per Layer }\end{array}$ & $\begin{array}{c}\text { Aluminum } \\
\text { Alloy } \\
\text { Thickness } \\
\text { [mm] }\end{array}$ & $\begin{array}{c}\text { Prepreg } \\
\text { Thickness } \\
\text { per } \\
\text { Orientation } \\
\text { [mm] }\end{array}$ & $\begin{array}{c}\text { Number } \\
\text { of } \\
\text { Cohesive } \\
\text { Layers }\end{array}$ & $\begin{array}{c}\text { Cohesive } \\
\text { Interface } \\
\text { Thickness } \\
\text { [mm] }\end{array}$ & $\begin{array}{c}\text { Total } \\
\text { Laminate } \\
\text { Thickness } \\
\text { [mm] }\end{array}$ \\
\hline $\begin{array}{c}\text { GLARE3- } \\
\text { 5/4-0.3 }\end{array}$ & $0^{\circ}$ & 0.33 & 0.125 & 12 & 0.001 & 2.662 \\
\hline $\begin{array}{c}\text { GLARE3- } \\
\text { 4/3-0.3 }\end{array}$ & $0^{\circ} / 90^{\circ}$ & 0.33 & 0.125 & 9 & 0.001 & 2.079 \\
\hline $\begin{array}{c}\text { GLARE4- } \\
\text { 3/2-0.3 }\end{array}$ & $90^{\circ} / 0^{\circ} / 90^{\circ}$ & 0.3 & 0.125 & 8 & 0.001 & 1.658 \\
\hline
\end{tabular}

Figure 4.1 shows the geometry used in the progressive damage FE models. More specifically, Figure 4.2 shows the modified lateral restraint as applied to the GLARE4 variant, including an increased slot for the application of the biaxial gages as per the experimentation. 


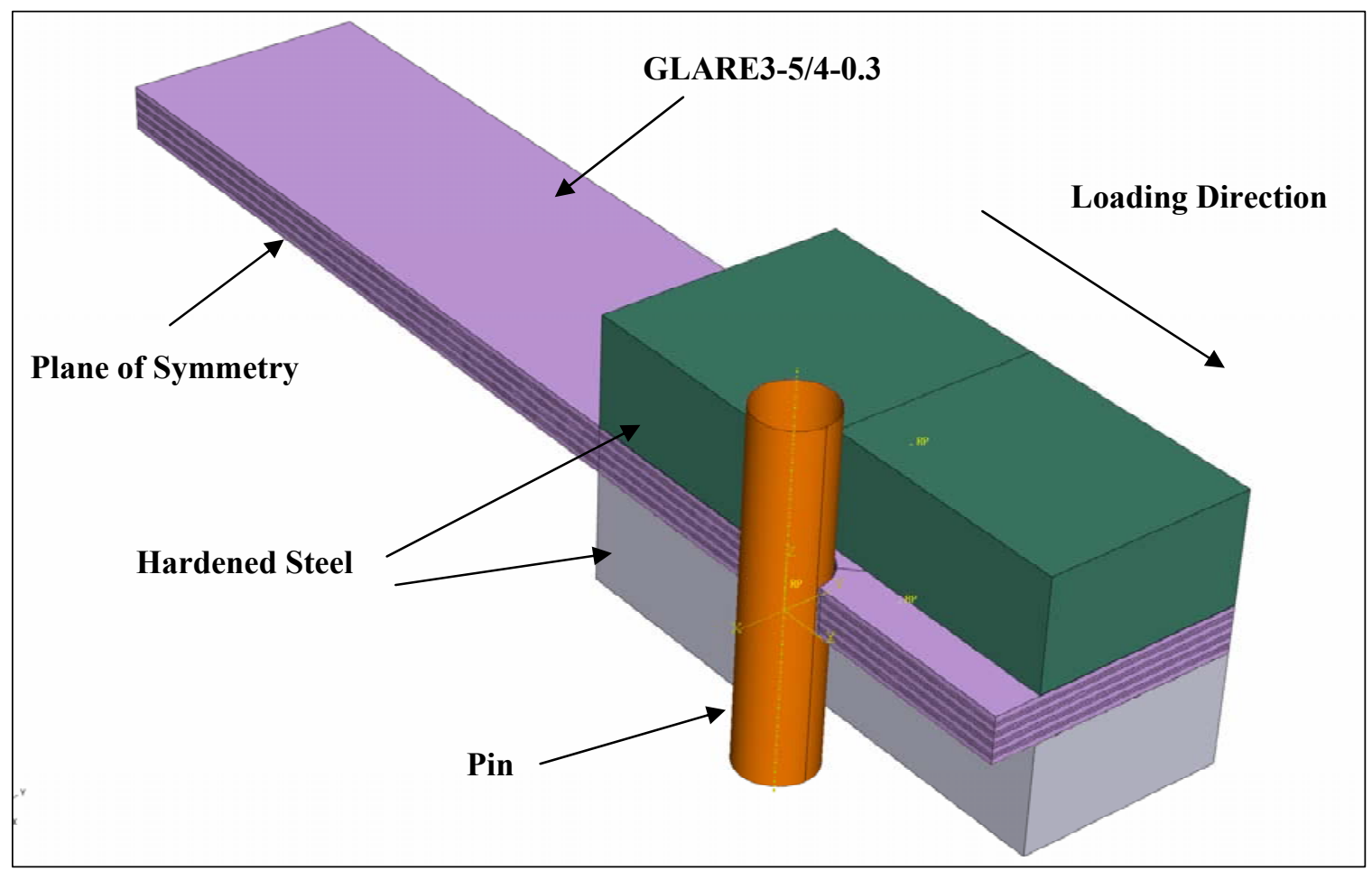

Figure 4.1: Global geometry of a typical model with loading pin and hardened steel lateral restraints.

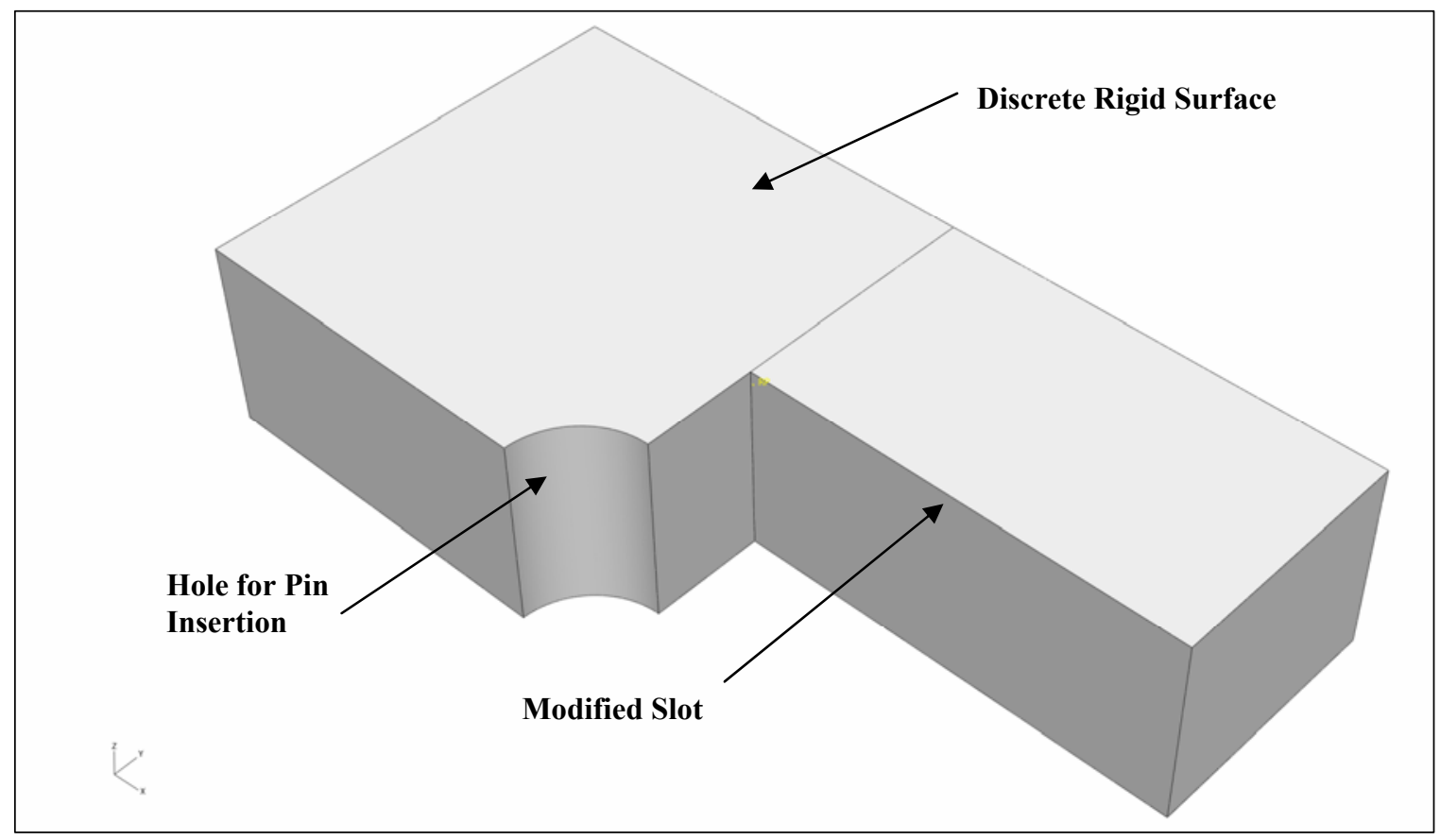

Figure 4.2: Modified hardened steel lateral restraint for application to the GLARE4-3/2-0.3 specimen highlighting the modified machined slot. 
In all three of the variants analyzed, a gap of $0.0275 \mathrm{~mm}$ was left between the lateral restraints and the laminate itself to simulate the space left via the tensile loading fixture of the experiments. Interactions between the laminate and the pin as well as the laminate and the lateral restraints were modeled with a surface-to-node contact algorithm for the detection and calculation of normal contact forces. Friction was modeled with a coefficient of friction set equal to 0.1 for both the pin-laminate and restraint-laminate interactions.

\subsection{Material Considerations and Implementation}

\subsubsection{4-T3 Aluminum with Isotropic Strain Hardening}

The main material in GLARE and that of principal interest for the YDB mechanism is that of 2024-T3 aluminum, modeled to include isotropic strain hardening for the onset of plasticity. This inclusion of isotropic strain hardening for the aluminum is important since in the event that the applied load approaches the yield strength of the aluminum, its behaviour and stiffness will vary (in this case its stiffness increases) such that any result subsequent to this loading may be erroneous if not included in the present analysis. In addition, since one of the main focuses of this study is the detection of yielding in the aluminum, at what distance this occurs from the fastener hole and how it is related to the propagation and ending of delamination as part of the proposed YDB mechanism, it is of critical importance that the effect of the onset of plasticity is included. 
To that end, strain hardening and plasticity data, as shown in Table 4.2, were implemented for a maximum strain of $25 \%$ after which the modulus remains constant and the material has undergone severe plastic deformation ie. failed. Values above $17 \%$ were extrapolated from stress-strain data available in the literature and were required to ensure the numerical stability of the simulation [89]. Parameters for the aluminum were implemented employing the *ELASTIC and *PLASTIC keywords in ABAQUS [112].

Table 4.2: Elastic and plastic properties of 2024-T3 aluminum.

\begin{tabular}{|c|c|}
\hline \multicolumn{2}{|c|}{ Elastic Properties } \\
\hline Elastic Modulus [MPa] & Poisson's Ratio [/] \\
\hline 72400 & 0.33 \\
\hline \multicolumn{2}{|c|}{ Plastic Properties } \\
\hline Yield Strength [MPa] & Plastic Strain [\%] \\
\hline 300.00 & 0.0 \\
\hline 320.00 & 0.016 \\
\hline 340.00 & 0.047 \\
\hline 355.00 & 0.119 \\
\hline 375.00 & 0.449 \\
\hline 390.00 & 1.036 \\
\hline 410.00 & 2.130 \\
\hline 430.00 & 3.439 \\
\hline 450.00 & 5.133 \\
\hline 470.00 & 8.00 \\
\hline 484.00 & 14.710 \\
\hline 490.00 & 17.00 \\
\hline 496.15 & 18.00 \\
\hline 507.11 & 25.00 \\
\hline
\end{tabular}




\subsubsection{FM-94 Epoxy Adhesive: Elastic Response, Damage Initiation, Evolution and Failure Law}

The constitutive response and ultimate failure of the adhesive system employed was modeled using cohesive elements based on a traction-separation law for elastic response as well as a damage initiation criterion. A subsequent damage evolution law, with the option of element deletion upon sufficient degradation was also employed. The details pertaining to the implementation of the cohesive elements and their damage evolution is discussed below.

\subsubsection{Implementation of a Traction Separation Law for a Cohesive Interface}

Cohesive behaviour defined directly in terms of a traction-separation law is primarily intended for bonded interfaces where the surface thickness is negligibly small and is used ideally for modeling the delamination at interfaces of composites by allowing the specification of material data such as fracture energy and multiple damage mechanisms. The stress state induced in a pin bearing case is complex and thus would give rise to a mixed mode delamination scenario including components of mode I, II and II. It has been confirmed in the literature that a quadratic failure criterion based on interlaminar stresses can effectively predict the nucleation of delamination damage and has been used extensively [106-110,118-121]. This quadratic failure criterion considers that the onset of delamination is not affected by compressive normal tractions and takes on the form of Equation (1.4):

$$
\left(\frac{\left\langle\tau_{3}\right\rangle}{N}\right)^{2}+\left(\frac{\tau_{2}}{S}\right)^{2}+\left(\frac{\tau_{1}}{T}\right)^{2}=1
$$


where $\tau_{\mathrm{i}}$ represents the applied tractions in the normal $\left(\tau_{3}\right)$ and two shear $\left(\tau_{2}, \tau_{1}\right)$ directions of the interface while $N, S$ and $T$ refer to the maximum allowable values in the same three interfacial directions.

The total mixed-mode relative displacement denoted by $\delta_{m}$, is defined in Equation (4.2) with $\delta_{3}$ corresponding to the normal displacement and $\delta_{1}$ and $\delta_{2}$ correspond to the inplane shear deformations:

$$
\delta_{m}=\sqrt{\delta_{1}^{2}+\delta_{2}^{2}+\left\langle\delta_{3}\right\rangle^{2}}=\sqrt{\delta_{\text {shear }}^{2}+\left\langle\delta_{3}\right\rangle^{2}}
$$

Here, the $\delta_{\text {shear }}$ term represents the norm of the vector defined by the two in-plane shear terms.

A penalty stiffness, $K$, can then be applied to relate the applied traction and induced displacement, before softening in all three modes via Equation (4.3):

$$
\tau_{i}=K \delta_{i}, \mathrm{i}=1,2,3
$$

It is then assumed that at the onset of softening (delamination damage), the two in-plane shear allowables are equal to each other (i.e. $S=T$ ) and thus the relative displacements in each mode can be described in terms of the allowable and the penalty stiffness as shown in Equation (4.4) and Equation (4.5):

$$
\begin{gathered}
\delta_{3}^{o}=\frac{N}{K} \\
\delta_{1}^{o}=\delta_{2}^{o}=\delta_{\text {shear }}^{o}=\frac{S}{K}
\end{gathered}
$$


Finally, the mixed-mode relative displacement corresponding to the onset of softening, $\delta_{m}{ }^{o}$, can be obtained through the direct substitution and algebraic manipulation of Equation (4.2) - Equation (4.5) into Equation (1.4) and isolating $\delta_{m}$ to yield:

$$
\delta_{m}^{o}=\left\{\begin{array}{c}
\delta_{3}^{o} \delta_{1}^{o} \sqrt{\frac{1+\beta^{2}}{\left(\delta_{1}^{o}\right)^{2}+\left(\beta \delta_{3}^{o}\right)^{2}}}, \quad \delta_{3}>0 \\
\delta_{\text {shear }}^{o}, \quad \delta_{3} \leq 0
\end{array}\right.
$$

where $\delta_{1}{ }^{o}, \delta_{2}{ }^{o}$ and $\delta_{3}{ }^{o}$ are the single mode softening onset displacements and the parameter $\beta$ is the mode-mixity ratio defined by Equation (4.7):

$$
\beta=\frac{\delta_{\text {shear }}}{\delta_{3}}
$$

The result of the preceding derivation, with the intermediate steps performed by the author, is a quadratic failure criterion based on maximum allowable traction and mixedmode delamination [107-109].

The implementation of a traction-separation elastic response for a cohesive interface in ABAQUS via Python, requires the specification of an elastic modulus in a direction normal to the element surfaces and two shear moduli in a longitudinal and transverse sense within the element surfaces. ABAQUS assumes as its default a constitutive thickness of $1 \mathrm{~mm}$ such that no distinction is required between the separation displacement and the nominal strain. As a result, the specified moduli must be scaled down by a factor $t$ to represent the actual thickness of the modeled interface based on its proportion to the default thickness, which in the case of this study was set to 0.001 . The elastic parameters are calculated via the following equations [112]: 


$$
\begin{aligned}
& K_{n n}=\frac{E}{t} \\
& K_{s s}=\frac{G}{t} \\
& K_{t t}=\frac{G}{t}
\end{aligned}
$$

For the situation at hand, the required material parameters for the FM-94 epoxy adhesive were: $\mathrm{E}=2000 \mathrm{MPa}$ and $v=0.28$. The shear modulus for the adhesive was calculated via the well known relationship between elastic modulus and shear modulus given by Equation (4.11):

$$
G=\frac{E}{2(1+v)}
$$

The calculated value for the shear modulus of the adhesive interface was found to be $G=$ 996.09 MPa. Keeping in mind the aforementioned values for elastic modulus and the newly calculated shear modulus as well as the actual thickness of the cohesive interface, the parameters required for the specification of the traction-separation elastic response of the adhesive were calculated and are summarized in Table 4.3. These values were implemented using the *ELASTIC, type $=$ TRACTION keywords in ABAQUS [112].

Table 4.3: Calculated elastic parameters for the traction-separation response of FM94 epoxy adhesive.

\begin{tabular}{cc}
\hline Parameter & Calculated Value [MPa] \\
\hline $\mathrm{K}_{\mathrm{nn}}$ & 12750.0 \\
\hline $\mathrm{K}_{\mathrm{ss}}$ & 4980.47 \\
\hline $\mathrm{K}_{\mathrm{tt}}$ & 4980.47 \\
\hline
\end{tabular}




\subsubsection{Implementation of Damage Initiation for the Cohesive Interface}

A quadratic nominal strain criterion was used to model the mixed-mode damage initiation within the cohesive interface. Damage is assumed to initiate when a quadratic interaction function involving the nominal strain reaches a value of one. The implementation of the criterion requires the specification of three nominal strain values, namely, a normal-only mode, a shear-only mode in the first shear direction and a shear-only mode in the second shear direction. The calculation of these nominal values requires the specification of a failure stress or maximum allowable traction, in the same three directions. Nominal strain, in this case, refers to its conventional meaning of far field, minimal values. Numerous values for the allowable tractions exist in the literature for the employed FM94 epoxy in GLARE. Unfortunately, these vary tremendously in their values and it has been reported to be as low as $20 \mathrm{MPa}$ [57] for the normal traction allowable through to values of $102 \mathrm{MPa}[85]$ and even higher. Such discrepancy in available allowable traction values has lead to difficulty in implementing delamination modeling and has been successfully addressed through the scaling down of these values such that delamination is allowed to occur [122].

For the present study, these values, resting on the assumption of an isotropic adhesive, we set to be:

$$
t_{n}^{f}=t_{s}^{f}=t_{t}^{f}=20 M P a
$$

The selected value of $20 \mathrm{MPa}$ for the maximum traction allowables represents the lowest values within the large range available in the literature as discussed [57]. Additional values were considered and implemented including $50 \mathrm{MPa}$ and $26.5 \mathrm{MPa}$ but it was 
found that the delamination was not allowed to initiate with such high values of interfacial strength. As mentioned previously, it has been reported that the maximum traction allowable values can be scaled down such that delamination is allowed to occur [122]. To remain conservative, the lowest reported value available was indeed implemented and thus the introduction of additional assumptions and subjectivity by virtue of the scaling down procedure, though shown to be effective in some cases, was not required.

Furthermore, it is these precise parameters which correspond to the normal and shear allowables depicted in Equation (4.12). From the maximum traction allowable values, the nominal strains were calculated via Equation (4.13) - Equation (4.15) where $t$ is the same thickness scaling factor used previously [112]:

$$
\begin{gathered}
\varepsilon_{n}^{f}=\frac{t_{n}^{f}}{E / t} \\
\varepsilon_{s}^{f}=\frac{t_{s}^{f}}{G / t} \\
\varepsilon_{t}^{f}=\frac{t_{t}^{f}}{E / t}
\end{gathered}
$$

Employing the previous maximum allowables as well as the elastic and shear moduli, the nominal strains for the normal and two shear directions were calculated and are tabulated in Table 4.4.

The nominal strains were implemented into ABAQUS using the *DAMAGE Initiation, criterion $=$ QUADE keywords [112]. 
Table 4.4: Nominal strain parameters required for the quadratic nominal strain criterion within the damage initiation model.

\begin{tabular}{cc}
\hline Parameter & Calculated Value [/] \\
\hline$\varepsilon_{n}^{f}$ & 0.00001 \\
\hline$\varepsilon_{s}^{f}$ & 0.0000266 \\
\hline$\varepsilon_{t}^{f}$ & 0.0000266 \\
\hline
\end{tabular}

This represents all the required parameters for the damage initiation criterion represented in Equation (1.4), which once satisfied i.e. reaches a value greater than or equal to 1, initiates the damage evolution law explained in the next section.

\subsubsection{Implementation of Damage Evolution for a Cohesive Interface}

Criteria developed to predict delamination propagation subject to mixed-mode loading configurations are typically established through the fracture mechanics parameters of strain energy release rate $G$ and fracture toughness $K$. More specifically, for a mixedmode loading configuration, there is an inherent dependence of the fracture toughness on the mode mix, which is accounted for in delamination propagation criteria.

The evolution of damage in this type of modeling is a progressive degradation of the stiffness associated with the traction-separation law employed to model the elastic response of the interface layer. The "softening" of the material can be chosen to be either linear or exponential in nature. In the case of the study at hand, an exponential softening behaviour was selected since it has been reported in the literature that, in fact, the behaviour of GLARE in this capacity resembles an exponential curve [87].

A mixed-mode criterion proposed by Benzeggagh and Kenane (the B-K criterion, herein) [105] has been shown to be able to accurately capture the mixed-mode fracture toughness 
over a large range (nearly comprehensive) of mode mixity ratios for numerous composite materials, making it a very applicable general criterion [105,107-109].

The B-K criterion is expressed as a function of an empirically derived parameter $\eta$ and both mode I and mode II critical strain energy release rates as depicted by Equation (4.16) [105]:

$$
G_{I C}+\left(G_{I I C}-G_{I C}\right)\left(\frac{G_{\text {shear }}}{G_{T}}\right)^{\eta}=G_{C} \text { with } \mathrm{G}_{\mathrm{T}}=\mathrm{G}_{\mathrm{I}}+\mathrm{G}_{\mathrm{II}}
$$

The semi-empirical form of Equation (4.16) was originally proposed to predict $G_{C}$ for glass/epoxy composites making it highly attractive for application to the present investigation of FMLs in a pin bearing loading configuration. The mixed-mode relative displacement corresponding to complete decohesion for the B-K criterion, represented by $\delta_{m}{ }^{f}$, is given by Equation (4.17) [105]:

$$
\delta_{m}^{f}=\left\{\begin{array}{c}
\frac{2}{K \delta_{m}^{o}}\left[G_{I C}+\left(G_{I I C}-G_{I C}\right)\left(\frac{\beta^{2}}{1+\beta^{2}}\right)^{\eta}\right], \quad \delta_{3}>0 \\
\sqrt{\left(\delta_{1}^{f}\right)^{2}+\left(\delta_{2}^{f}\right)^{2}}, \quad \delta_{3} \leq 0
\end{array}\right.
$$

This final relation represents a complete description of the mixed-mode relative displacement corresponding to total decohesion and requires the specification of the interface stiffness $K$, the critical energy release rates for the normal and two in-plane shear directions and finally, the semi-empirical parameter $\eta$.

Thus, the only parameter in the B-K criterion shown in Equation (4.16) which remains to be specified is the semi-empirical parameter $\eta$. Benzeggagh and Kenane [105] employed a curve fitting scheme to their double cantilever beam (DCB) data for a glass/epoxy composite and found that the calculated value of $\eta=2.6$ yields very good results. 
Camanho et al. [107-109] performed a similar curve fitting approach to an AS4/PEEK composite to produce a value of $\eta=2.284$ which also yielded good results.

Since production of empirical data on the critical strain energy release rates for a composite is an expensive and time consuming process, it was decided to employ the original data produced by Benzeggagh and Kenane herein [105]. However, since the curve fitting scheme used in their regression analysis was not specified in their results, a minimization of least squares error performed on that data, as proposed by Camanho et. al. [107] with the intermediate steps performed by the author, was employed herein. The identification of the problem consists of determining the parameter $\eta$ from a set of empirical data for which the B-K criterion (a polynomial expression as a function of $\left(\frac{G_{I I}}{G_{T}}\right)$ or if mode III is significant $\left(\frac{G_{\text {shear }}}{G_{T}}\right)$ ) has been shown to fit as in Equation (4.18) [107]:

$$
p\left(\frac{G_{I I}}{G_{T}}\right)=G_{I C}+\left(G_{I I C}-G_{I C}\right)\left(\frac{G_{I I}}{G_{T}}\right)^{\eta}
$$

If we consider the empirical data as a set of $n$ points of $\left(\left(\mathrm{G}_{\mathrm{II}} / \mathrm{G}_{\mathrm{T}}\right)_{\mathrm{i}},\left(\mathrm{G}_{\mathrm{T}}\right)_{\mathrm{i}}\right)$ the problem can be formulated thusly, as shown in Equation (4.19):

$$
f=\sum_{i=1}^{n}\left[\left(G_{T}\right)_{i}-G_{I C}+\left(G_{I I C}-G_{I C}\right)\left(\frac{G_{I I}}{G_{T}}\right)_{i}^{\eta}\right]^{2}
$$

By differentiating Equation (4.19) with respect to $\eta$, setting it equal to zero and then solving for $\eta$, we can arrive at an appropriate minimum value for the semi-empirical parameter, as expressed in Equation (4.20): 


$$
\sum_{i=1}^{n}\left[\left(G_{T}\right)_{i}-G_{I C}+\left(G_{I I C}-G_{I C}\right)\left(\frac{G_{I I}}{G_{T}}\right)_{i}^{\eta}\right]\left(\frac{G_{I I}}{G_{T}}\right)_{i}^{n} \ln \left(\frac{G_{I I}}{G_{T}}\right)_{i}=0
$$

The original strain energy release rate data provided by Benzeggagh and Kenane [105] is summarized and formatted for application into Equation (4.20) in Table 4.5 for a range of mode mixes in a pure mode I sense through to and including a pure mode II loading. A short routine written in Maple V10 software [114] performed the required analysis incorporating the data provided in Table 4.5 and produced a value of $\eta=2.668$. For reference, the employed routine can be found in the appendices.

Table 4.5: Mode-mixity and strain energy release rates as for a glass/epoxy composite [105].

\begin{tabular}{ccc}
\hline Mode(s) & $\boldsymbol{G}_{\|} / \mathbf{G}_{T}[\%]$ & $\boldsymbol{G}_{T c}\left[\mathrm{~J} / \mathbf{m}^{2}\right]$ \\
\hline II & 100 & $2905.76 \pm 224.55$ \\
\hline I \& II & 91 & $2457.26 \pm 100.30$ \\
\hline I \& II & 82 & $1821.93 \pm 84.47$ \\
\hline I \& II & 72 & $1033.67 \pm 174.11$ \\
\hline I \& II & 53 & $579.62 \pm 58.66$ \\
\hline I \& II & 43 & $568.36 \pm 98.58$ \\
\hline I \& II & 28 & $340.35 \pm 37.26$ \\
\hline I & 0 & $118.02 \pm 2.72$ \\
\hline
\end{tabular}

The newly calculated value is comparable to the $\eta=2.6$ value generated for glass/epoxy in Reference [105] using a different and unknown regression scheme, but the results are more than satisfactory. Therefore, the calculated semi-empirical value of $\eta=2.668$ will be employed for the all of the progressive damage FE analyses conducted herein. For implementation into ABAQUS, additional specification of the values of critical fracture energy required to propagate failure in the normal, first and second shear 
directions was also required. These values, again stemming from the assumption of isotropy within the interface, were assumed to be equal for each direction and take on the following values as shown in Equation (4.21):

$$
G_{n}^{c}=G_{s}^{c}=G_{t}^{c}=4 N / \mathrm{mm}
$$

With the all of the above values implemented, the damage initiation and evolution model for the interface layer of the adhesive is complete.

\subsection{3 $\mathrm{S}_{2}$-Glass Fibre Composite Prepreg with Damage Initiation and Evolution}

The implementation of material considerations for the $\mathrm{S}_{2}$-glass fibre prepreg was similar to the cohesive interface model with a few notable exceptions. First, the constitutive response for the elastic behaviour was not a traction-separation law and second, the assumption of plane stress was invoked.

\subsubsection{Undamaged Elastic Response}

Implementation of composite type materials in ABAQUS requires the specification of their undamaged material response employing one of the methods of defining an orthotropic, linear elastic material. The *ELASTIC, type = LAMINA keyword in ABAQUS was used and local material directions were specified by user defined coordinate axes such that the 1-axis (fibre direction) of the prepreg layers corresponded with the longitudinal direction of the model geometry. The specific values for the material constants themselves are summarized in Table 4.6. 
Table 4.6: Summary of the elastic properties of the $S_{2}$-glass prepreg layers.

\begin{tabular}{cc}
\hline Material Parameter & Value \\
\hline $\mathrm{E}_{1}[\mathrm{MPa}]$ & 55000 \\
\hline $\mathrm{E}_{2}[\mathrm{MPa}]$ & 9500 \\
\hline$v_{12}[/]$ & 0.33 \\
\hline $\mathrm{G}_{12}[\mathrm{MPa}]$ & 5500 \\
\hline $\mathrm{G}_{13}[\mathrm{MPa}]$ & 3000 \\
\hline $\mathrm{G}_{23}[\mathrm{MPa}]$ & 3000 \\
\hline
\end{tabular}

\subsubsection{Implementation of Damage Initiation for Elastic-Brittle Materials Exhibiting Anisotropic Behaviour}

Damage initiation and evolution can be modeled within ABAQUS for materials

exhibiting elastic-brittle failure and anisotropic behaviour. This type of failure is ideal for composite materials since damage in materials of this class is initiated with little or no plastic deformation and can thus be neglected with no adverse consequences. The criterion employed by ABAQUS is based on Hashin's theory and considers degradation at material or integration points within an element subject to four mechanisms namely: fibre tension, fibre compression, matrix tension and matrix compression. These four mechanisms lead to the calculation and evolution of four damage variables namely: $\mathrm{d}_{\mathrm{f}}^{\mathrm{t}}$, $\mathrm{d}_{\mathrm{f}}^{\mathrm{c}}, \mathrm{d}_{\mathrm{m}}^{\mathrm{t}}, \mathrm{d}_{\mathrm{m}}^{\mathrm{c}}$ which correspond to the previously mentioned modes of failure. In order to calculate the four damage variables, the analyst is required to specify values for the following variables:

$\mathrm{X}^{\mathrm{T}}$ which denoted tensile strength in the fibre direction;

$\mathrm{X}^{\mathrm{C}}$ which denotes compressive strength in the fibre direction;

$\mathrm{Y}^{\mathrm{T}}$ which denotes tensile strength in the transverse (perpendicular to the fibres) direction; $\mathrm{Y}^{\mathrm{C}}$ which denotes compressive strength in the transverse direction; 
$\mathrm{S}^{\mathrm{L}}$ which denotes the longitudinal shear strength;

$\mathrm{S}^{\mathrm{T}}$ which denotes the transverse shear strength;

In general, the value of $\mathrm{S}^{\mathrm{T}}$ is not truly independent and is most often expressed in Hashin's theory as calculated by Equation (4.22):

$$
S^{T}=\frac{Y^{C}}{2}
$$

Table 4.7 provides a summary of the values used in the model for the glass prepreg layers. Implementation of Hashin's theory within ABAQUS requires that the plane stress assumption be valid, which for the case of the geometry in question is without consequence since the model employs thin layers within the laminate.

Table 4.7: Summary of the failure strengths employed for the glass prepreg layers.

\begin{tabular}{cc}
\hline Material Parameter & Value [MPa] \\
\hline $\mathrm{X}^{\mathrm{T}}$ & 2500 \\
\hline $\mathrm{X}^{\mathrm{C}}$ & 2000 \\
\hline $\mathrm{Y}^{\mathrm{T}}$ & 50 \\
\hline $\mathrm{Y}^{\mathrm{C}}$ & 150 \\
\hline $\mathrm{S}^{\mathrm{L}}$ & 50 \\
\hline $\mathrm{S}^{\mathrm{T}}$ & 75 \\
\hline
\end{tabular}

Encoding of the failure theory in ABAQUS was accomplished using the *DAMAGE INITIATION, CRITERION $=$ HASHIN, ALPHA $=$ VALUE keyword where the variable alpha refers to a coefficient that determines the contribution of shear stresses to the onset of fibre failure in tension [112]. For the purposes of this study, the coupling parameter alpha was set to the common value of 0.0 , indicating no contribution of shear stress to the onset of tensile failure in the fibres. This is a simplifying and conservative assumption. 
However, since the pin bearing loading configuration leads to stresses with a compressive sense and failure will occur in that light, it is felt that is a reasonable assumption to make and analysis based upon it can be carried out with confidence.

As the damage criteria are met, the material properties corresponding to each mode are degraded in an analogous fashion to the cohesive elements discussed previously using a damage evolution law, which will be presented next.

\subsubsection{Implementation of Damage Evolution for Fibre-Reinforced Composites Exhibiting Elastic-Brittle Behaviour}

The evolution of damage for elastic-brittle material behaviour operates on a similar governing principle as the traction-separation based law for cohesive interfaces; namely, that the damage is characterized by a progressive degradation of material stiffness $i e$. softening, which ultimately leads to failure of the material. It requires that the undamaged response of the material is linearly elastic with no plasticity effects included. The decay in material stiffness was assigned to behave linearly in order to improve convergence, as recommended by ABAQUS [112].

Damage is assumed to evolve due to dissipation in energy throughout the damage process and therefore the analyst is required to supply values for the critical energy release rate for all of the possible damage modes. In this case it requires the specification of the critical energy release rates for the fibres in tension and compression and for the matrix in tension and compression denoted $G_{f}^{T}, G_{f}^{C}, G^{T}{ }_{m}$ and $G^{C}{ }_{m}$ respectively. Precise values of these variables were not available to the author at the time of the analysis and of this writing and conducting energy release rate experiments would be prohibitively costly, therefore in an effort to avoid arbitrary assignment of said values, a benchmark example 
provided in the ABAQUS User's manual regarding glass fibre composites was used as a source [112]. Furthermore, to simplify the analysis, reduce computational time and as is the expectation from the ABAQUS User's manual, no differentiation was made between tension and compression for the specification of these values for input. This once again stems from the consequences of the pin bearing loading configuration and its bias towards compressive loading. The precise values employed in the analysis are summarized in Table 4.8. The encoded implementation of the damage evolution law was accomplished through the $*$ Damage Evolution, type $=$ ENERGY keyword in ABAQUS [112].

Table 4.8: Critical energy release rates for the fibre and matrix in both tension and compression [112].

\begin{tabular}{cccc}
\hline $\begin{array}{c}\text { Fibre Tensile } \\
\text { Fracture Energy } \\
{[\mathrm{N} / \mathbf{m m}]}\end{array}$ & $\begin{array}{c}\text { Fibre Compressive } \\
\text { Fracture Energy } \\
{[\mathbf{N} / \mathbf{m m}]}\end{array}$ & $\begin{array}{c}\text { Matrix Tensile } \\
\text { Fracture Energy } \\
{[\mathbf{N} / \mathbf{m m}]}\end{array}$ & $\begin{array}{c}\text { Matrix Compressive } \\
\text { Fracture Energy } \\
{[\mathbf{N} / \mathbf{m m}]}\end{array}$ \\
\hline 12.5 & 12.5 & 1.0 & 1.0 \\
\hline
\end{tabular}

\subsection{Implementation of Thermally Induced Residual Stresses}

As mentioned previously nearly all - certainly for mechanical fastening - investigations into modeling the mechanical behaviour of GLARE have not included the residual stresses induced as a result of the curing process. Therefore it is proposed that they too are included in the progressive damage delamination FE models which will be discussed in the next section as part of the practical damage prediction methodology. 
The residual stresses induced during the cooling down phase post-curing are a direct consequence of the net difference in the coefficients of thermal expansion associated with each constituent material. Since the coefficient of thermal expansion for aluminum is larger than that of the glass fibres, the net result of the cooling is a tensile residual stress in the aluminum layers and a compressive residual stress in the glass layers. It is well known that a tensile residual stress is unfavourable in fatigue loading configurations, as well as contributor to delamination and therefore it is common to post stretch GLARE laminates by approximately $1 \%$ to relax some of these detrimental stresses [27]. However, even with the post-stretching applied, invariably, there will still be some residual stresses present. Using superposition, the actual stresses in the aluminum layers of a typical GLARE laminate is therefore the sum of the applied mechanical stress and the induced residual stresses. Their magnitude is not nominal and studies have been performed in order to measure or calculate their value $[35,38,86,123]$. The aim of this portion of the study is to implement the induced residual stresses into FE analysis, yet it is still valuable to retain an analytical procedure for their calculation in order to serve as a comparative means for the FE results. In addition, it is assumed for the analytical calculations that the laminates do not contain any holes or other geometric discontinuities.

The calculation of residual stresses in the aluminum layers rests on the assumption that they are the by-product of the curing process and not induced by other means (mechanical chiefly) such as work hardening by shot peening. The curing temperature for a GLARE FML is approximately 120 degrees Celsius (393 K) and the resulting stress in the aluminum layers can be calculated in two fashions. 
The first method represents the simplified approach for determining the tensile residual stress in the aluminum layers by assuming isotropy in the adjacent glass layers. As a consequence, it does not incorporate the possible changes in expansion between the longitudinal and transverse fibre layers and assumes that since glass is an insulator, this change in expansion from one direction to another is small and can therefore be grouped together into a single term for the expansion of the glass layers. This simplified approach is expressed in Equation (4.23) where $E_{A l}$ is the modulus of elasticity for aluminum; $E_{g l}$ represents the stiffness of the glass fibres in the longitudinal direction; $\alpha_{A l}$ is the coefficient of thermal expansion for aluminum; $\alpha_{g l}$ represents the single coefficient of thermal expansion for the glass fibre layers and $\Delta T$ represents the difference between curing temperature and room temperature responsible for the residual stress [38]:

$$
\sigma_{r, A l}=\frac{E_{A l} E_{g l}\left(\alpha_{A l}-\alpha_{g l}\right) \Delta T}{E_{A l}\left(1-\alpha_{g l} \Delta T\right)+E_{g l}\left(1-\alpha_{A l} \Delta T\right)}
$$

The corresponding compressive stress induced in the glass layers can be found via Equation (4.24) where $t_{A l, t o t}$ is the total thickness of all the aluminum layers; $t_{g l, 0, t o t}$ is the total thickness of all the glass fibre layers in the longitudinal direction; $t_{g l, 90, t o t}$ is the total thickness of all the glass fibre layers in the transverse direction and $\sigma_{r, A l}$ is the previously calculated tensile residual stress in the aluminum layers.

$$
\sigma_{r, g l}=-\frac{\sigma_{r, A l} t_{A l, t o t}}{t_{g l, 0, t o t}+t_{g l, 90, t o t}}
$$

The second method represents a more complete approach for determining the tensile residual stress in the aluminum by taking into account the directionality of all the 
constituents and assumes orthotropic behaviour of the glass layers as expressed in Equation (4.25) [27]:

$$
\begin{aligned}
& \sigma_{r, A l}=\left\lfloor t_{A l l, t o t} E_{A l}\left(\alpha_{A l}-\alpha_{A l}\right)+t_{g l, 0, t o t} E_{g l, 0}\left(\alpha_{g l, 0}-\alpha_{A l}\right)\right. \\
& \left.+t_{g l, 90, t o t} E_{g l, 90}\left(\alpha_{g l, 90}-\alpha_{A l}\right)\right] \cdot \frac{E_{A l}\left(T_{R}-T_{\text {curre }}\right)}{E_{\text {lam }}\left(t_{A l, t o t}+t_{g l, 0, t o t}+t_{g l, 90, \text { tot }}\right)}
\end{aligned}
$$

Here $E_{A l}$ is the modulus of elasticity for aluminum; $E_{g l, 0}$ is the modulus of elasticity for the glass fibres in the longitudinal direction; $E_{g l, 90}$ is the modulus of elasticity for the glass fibres in the transverse direction; $E_{\text {lam }}$ is the modulus of elasticity for the entire laminate (which can be calculated from a metal volume fraction approach in a smeared properties sense); $\sigma_{r, A l}$ is the residual stress in the aluminum layers due to curing; $t_{A l, t o t}$ is the total thickness of all the aluminum layers; $t_{g l, 0, t o t}$ is the total thickness of all the glass fibre layers in the longitudinal direction; $\mathrm{t}_{g l, 90, \text { tot }}$ is the total thickness of all the glass fibre layers in the transverse direction; $T_{\text {cure }}$ is the curing temperature used to make the laminate; $T_{R}$ is room temperature; $\alpha_{A l}$ is the coefficient of thermal expansion for aluminum; $\alpha_{g l, 0}$ is the coefficient of thermal expansion for the glass fibres in the longitudinal direction and $\alpha_{g l, 90}$ is coefficient of thermal expansion for the glass fibres in the transverse direction.

The corresponding compressive residual stress in the glass layers oriented longitudinally can be found through a rearrangement of the variables found in Equation (4.25) as shown in Equation (4.26):

$$
\begin{aligned}
& \sigma_{r, g l, 0}=\left\lfloor t_{A l l, t o t} E_{A l}\left(\alpha_{A l}-\alpha_{g l, 0}\right)+t_{g l, 0, t o t} E_{g l, 0}\left(\alpha_{g l, 0}-\alpha_{g l, 0}\right)\right. \\
& \left.+t_{g l, 90, t o t} E_{g l, 90}\left(\alpha_{g l, 90}-\alpha_{g l, 0}\right)\right] \cdot \frac{E_{g l, 0}\left(T_{R}-T_{\text {cure }}\right)}{E_{\text {lam }}\left(t_{A l, t o t}+t_{g l, 0, t o t}+t_{g l, 90, t o t}\right)}
\end{aligned}
$$


Through the same rearrangement scheme, the corresponding stress in the glass layers oriented transversely can be found via Equation (4.27):

$$
\begin{aligned}
& \sigma_{r, g l, 90}=\left\lfloor t_{A l, t o t} E_{A l}\left(\alpha_{A l}-\alpha_{g l, 90}\right)+t_{g l, 0, t o t} E_{g l, 0}\left(\alpha_{g l, 0}-\alpha_{g l, 90}\right)\right. \\
& \left.+t_{g l, 90, t o t} E_{g l, 90}\left(\alpha_{g l, 90}-\alpha_{g l, 90}\right)\right] \cdot \frac{E_{g l, 90}\left(T_{R}-T_{\text {cure }}\right)}{E_{l a m}\left(t_{A l, t o t}+t_{g l, 0, t o t}+t_{g l, 90, t o t}\right)}
\end{aligned}
$$

The aforementioned effective laminate modulus of elasticity $E_{\text {lam }}$, employed in Equation (4.25) can be calculated using a rule of mixtures, incorporating influence from both the aluminum and glass constituents as given by Equation (4.28):

$$
E_{l a m}=\frac{E_{A l} t_{A l, t o t}+E_{g l, 0} t_{g l, 0, t o t}+E_{g l, 90} t_{g l, 90, t o t}}{t_{A l, t o t}+t_{g l, 0, t o t}+t_{g l, 90, t o t}}
$$

To facilitate the calculation of residual stresses for both the former and the latter approaches, the required material properties are summarized in Table 4.9.

Values calculated via the simplified approach can be found in the literature [35] and place the range of calculated stresses in the aluminum layers to be in the neighbourhood of 25 $30 \mathrm{MPa}$.

Hoflsagare [123] reported empirically measured values for the aluminum in the same range while de Vries et. al. [86] report a $72 \mathrm{MPa}$ compressive residual stress in the glass prepreg layers. Since the aim of this study is to incorporate the residual stresses into the progressive damage FE analyses, both aforementioned approaches were employed and compared to the results of the FE analysis and empirical values.

A distinct analysis step was also implemented into the models to develop the residual stress state as a function of a predefined temperature field with a net change equal to $100^{\circ} \mathrm{C}$ - typical for FML fabrication. The temperature analysis step was set to occur prior 
Table 4.9: Summary of the properties required to calculate the residual stresses

\begin{tabular}{cc}
\hline Material Parameter & Value \\
\hline $\mathrm{E}_{\mathrm{Al}}$ & $72.40 \mathrm{GPa}$ \\
\hline $\mathrm{E}_{\mathrm{gl}}$ & $53.98 \mathrm{GPa}$ \\
\hline$\alpha_{\mathrm{Al}}$ & $21.6 \times 10^{-6} / \mathrm{K}$ \\
\hline$\alpha_{\mathrm{gl}}$ & $7-11 \times 10^{-6} / \mathrm{K}$ \\
\hline$\Delta \mathrm{T}$ & $100 \mathrm{~K}$ \\
\hline $\begin{array}{c}\text { Additional Material Parameters for Glass } \\
\text { Layers }\end{array}$ & Value \\
\hline $\mathrm{E}_{11}$ & $53980.0 \mathrm{MPa}$ \\
\hline $\mathrm{E}_{22}$ & $9412.0 \mathrm{MPa}$ \\
\hline $\mathrm{E}_{33}$ & $9412.0 \mathrm{MPa}$ \\
\hline$\alpha_{11}$ & $6.1 \times 10^{-6} / \mathrm{K}$ \\
\hline$\alpha_{22}$ & $2.62 \times 10^{-6} / \mathrm{K}$ \\
\hline$\alpha_{33}$ & $2.62 \times 10^{-6} / \mathrm{K}$ \\
\hline
\end{tabular}

to the applied loading step in an effort to simulate that the material arrives already prestressed before any mechanical stresses are applied via the pin bearing load. Encoding of the material parameters employed the *EXPANSION, type = ISOTROPIC for an even distribution of the applied temperature field [112].

\subsection{Meshing Considerations}

Meshing of the developed models consisted of several steps. Since the principal region of interest was the area next to the pin hole there was no need to mesh the entire models with high refinement. Therefore, portions of the model which were far from the region of interest were meshed in a more coarse, yet still uniform manner. Analysis checks and verification of element aspect ratios and internal angles were performed in order to confirm that the elements were not skewed in any way. 
Regions moving progressively closer to the area of interest required additional refinement of the mesh and this was accomplished using a strict biasing of mesh seeding of the edges and partitions leading towards the pin hole.

Figure 4.3 through Figure 4.6 show the global assembly mesh as well as the mesh for the individual layers. Since the inter-specimen geometry was identical in an in-plane sense and only differed in the thickness of aluminum, number and configuration of layers and the total number of layers, the same edge biasing scheme was employed for all the specimens examined. Mesh independency of the results was assured through convergence testing and it was enforced that only a single element through the thickness would be used to simulate each individual constituent layers.

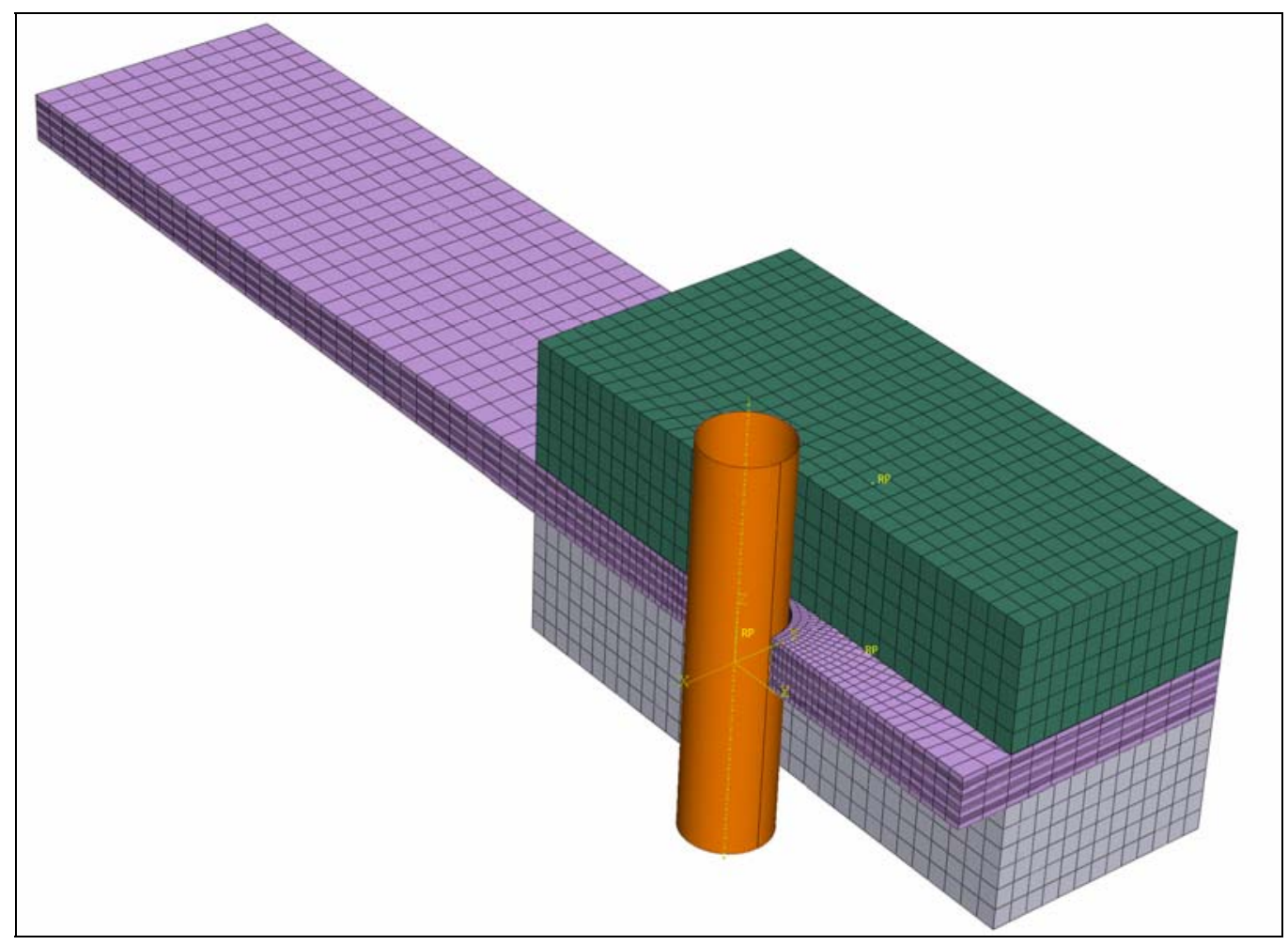

Figure 4.3: Global assembly mesh for a typical variant analyzed. 


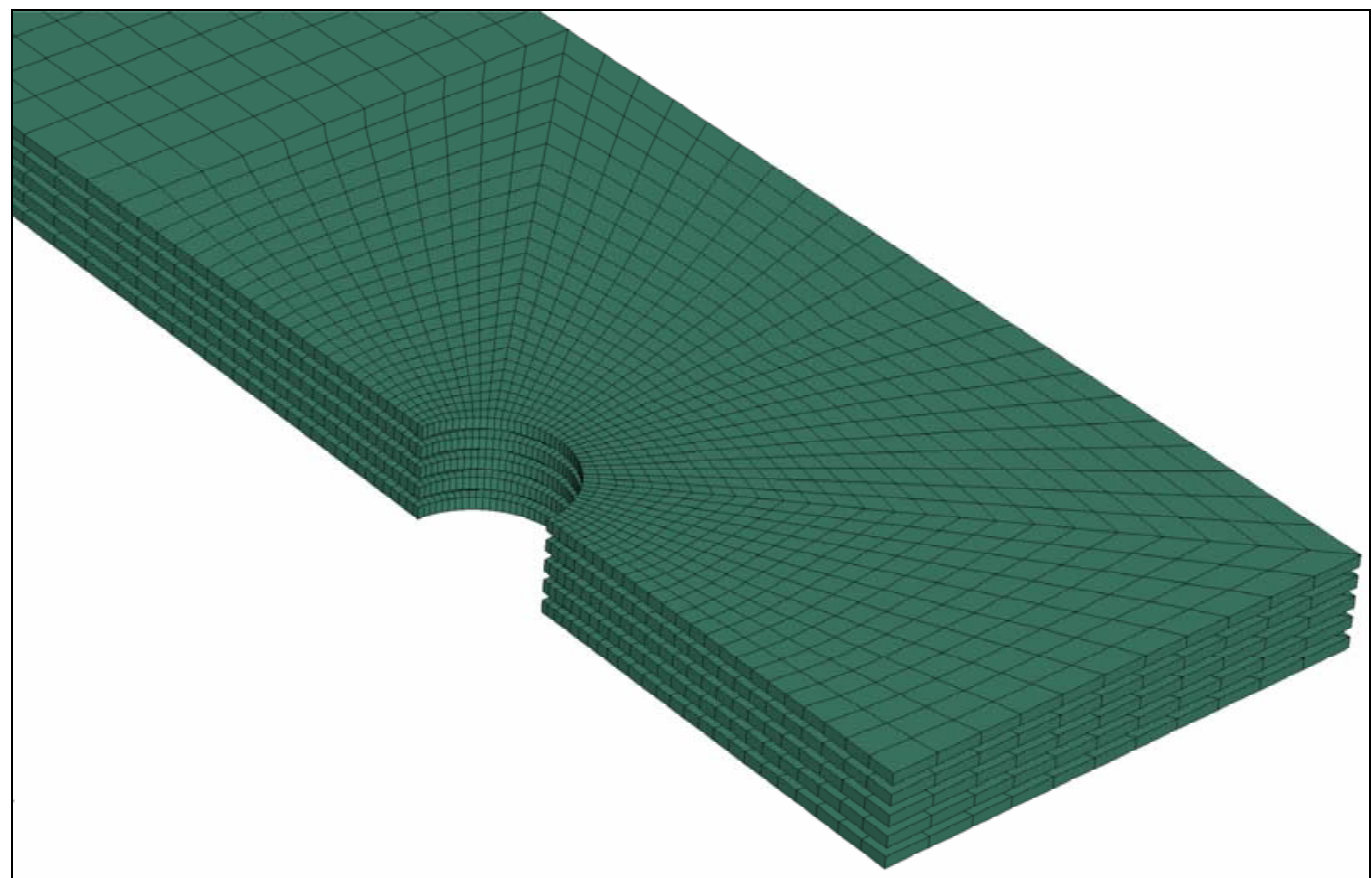

Figure 4.4: Typical local mesh for the aluminum layers only.

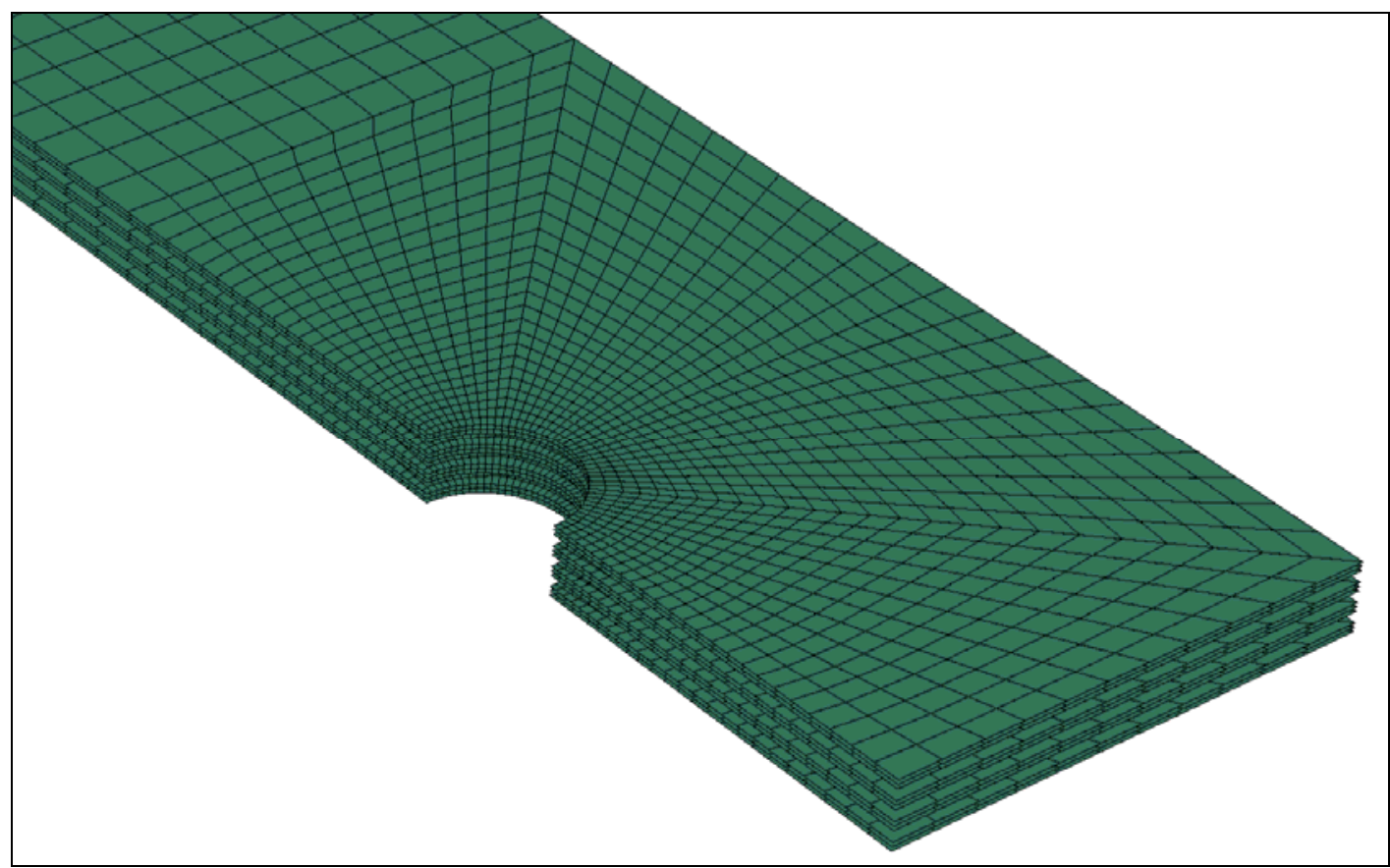

Figure 4.5: Typical local mesh for the cohesive interface layers. 


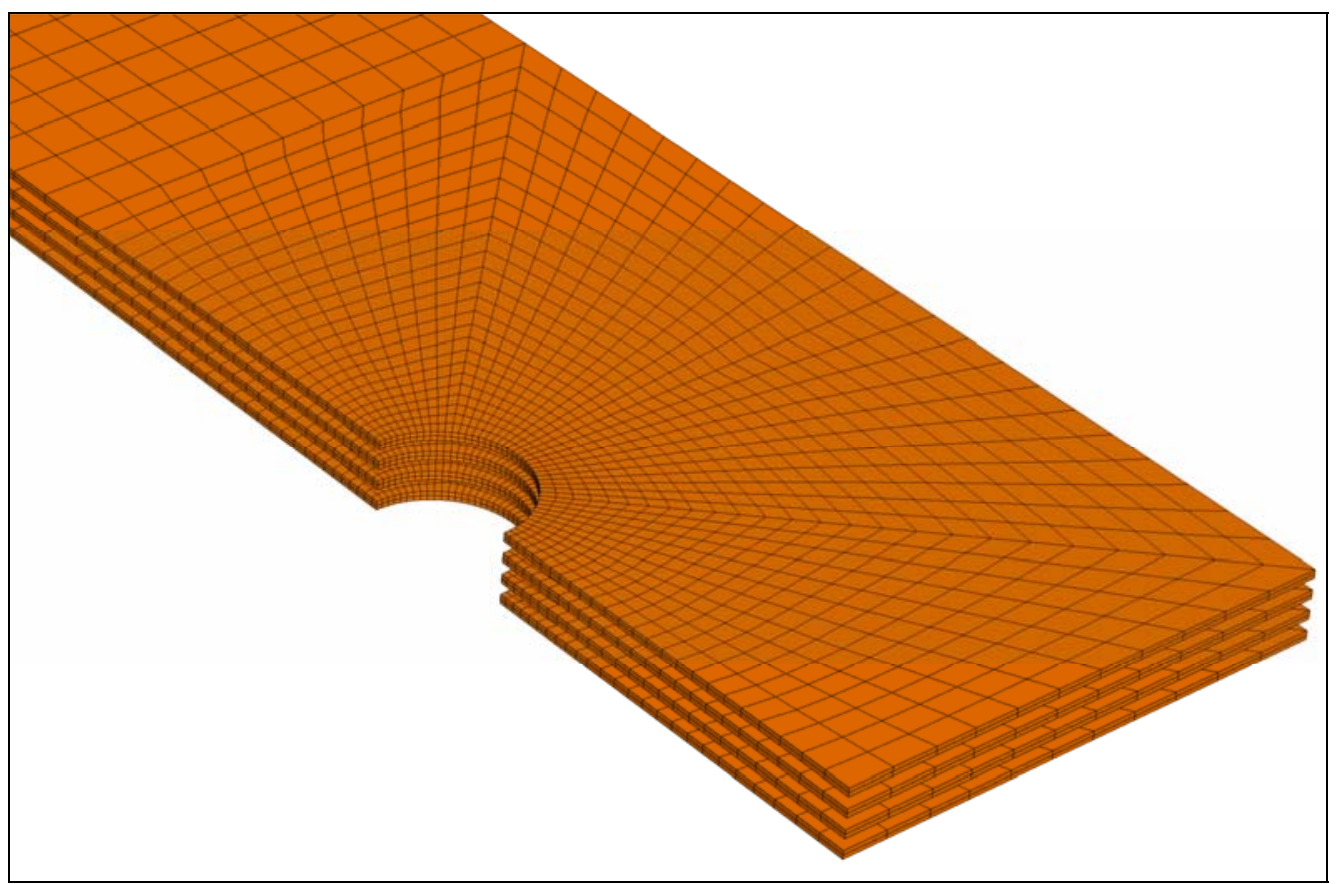

Figure 4.6: Typical local mesh for the glass prepreg layers.

\subsubsection{Meshing Considerations for the Aluminum Layers}

The elements chosen for the aluminum layers were of two varieties. The first were C3D8I incompatible mode hexahedral elements. This element type enhances the response of first order elements in bending by allowing internal, incompatible modes of deformation which help eliminate parasitic shear stresses that can make linear hexahedral elements overtly stiff in bending. Since the applied pin bearing loading may lead to bending in the layers in the through the thickness direction it is a good idea to employ elements that behave well in response to that loading configuration. By virtue of their definition, incompatible mode elements contain additional degrees of freedom that are computationally more expensive than first order elements and use full integration in the calculation of displacement. However, incompatible mode elements perform almost as 
well as quadratic elements with a significantly lower computational cost, if and only if, the elements are nearly rectangular with aspect ratios approaching 1.0. Though the applied meshing methodology through biased seeding and geometric considerations was performed carefully, it was not possible to lower the highest element aspect ratio below 3.0. The consequence of this is an increase in computational time required to analyze a case using incompatible mode elements. That being said and in an effort to eliminate introduced complexities, C3D8R, 8 node, linear reduced integration hexahedral elements were also retained in separate analyses for each variant studied.

\subsubsection{Meshing Considerations for the Cohesive Interface}

The adhesive interface of the models was also meshed with the same biased scheme incorporating high refinement in the region of interest. Since the most compliant section of the model will be the interface it therefore may be subject to high strains further emphasising the need for a properly designed mesh as conducted. In addition, since a damage initiation and evolution model has been implemented for the purpose of modeling decohesion, in the event of the applied stresses exceeding the shear strength of the adhesive, it is important to employ a highly refined mesh to accurately model the localized effects of the induced damage.

Adhesive interfaces in composite materials are frequently modeled - and indeed herein as well - in three dimensions using COH3D8 cohesive elements which can be thought of as being composed of two surfaces separated by a stipulated thickness. It is the relative motion of the top and bottom surfaces which dictates the transverse shear behaviour of the cohesive element as well as its through the thickness behaviour. This is what makes 
the use of cohesive elements valuable in the modeling of decohesion between adjacent faces of a material that is bonded together with an adhesive.

Cohesive and plane stress elements with severe damage can be deleted during an analysis and in fact that is the default criterion imposed by ABAQUS. The upper bound for the overall damage variable can be set by the analyst and commonly takes a value of 1.0. Once the overall damage variable has reached the assigned maximum for all the material points within the element and none of its material points are in compression, the element is deemed failed and can be removed. There is a large associated risk with element removal since once removed, the element offers no resistance to subsequent penetration of components and depending upon the model geometry and loading configuration, it may be necessary to model contact between the components formerly tied together by the failed cohesive element. As will be discussed in subsequent sections, the existing models already include significant non-linearity due to several enforced contact conditions of the pin and lateral restraints. This fact greatly increases the required computational time, in conjunction with the material degradation models included and would border on the prohibitive should additional contact be included. Also, the current geometry of the models in question does not run a risk for this penetrative problem and thus contact between adjacent layers already bonded by adhesive was not included in the modeling.

\subsubsection{Meshing Considerations for the Glass Prepreg Layers}

The composite prepreg layers in the model represent a typical plane stress situation in which the thickness of the section is significantly smaller than the remaining in-plane dimensions. It is quite common to model a plane stress scenario like this by using 
conventional shell elements, due to their increased computational efficiency and lack of through the thickness effects. In general, the analyst defines the thickness of the shell element by the specification of a value in the property module of ABAQUS. In contrast, the analyst could also employ continuum shell elements (akin to the solid-like shell elements discussed in the review section) by generating a solid part and allowing the software to determine the thickness of the shell as a virtue of the nodal geometry in addition to the analyst specified value. This two-fold approach keeps the definition of the shell geometry more "honest" and allows for easier modeling and viewing of a laminated structure since it incorporates a tangible, solid geometry. Continuum shell elements resemble their solid, three dimensional counterparts such as the previously discussed C3D8I and C3D8R elements in a geometrical perspective but behave in a constitutive and kinematic manner like conventional shells. Unlike conventional shells however, continuum shells do not have rotational degrees of freedom and only contain translational degrees of freedom much like continuum solid elements. SC8R, continuum shell elements were thus employed which are 8 node, quadrilateral, inplane, general purpose elements with reduced integration for increased computational efficiency. Care must be exercise by the analyst when employing continuum shell elements since they contain an associated thickness and normal direction that must be ordered consistently within the mesh. This problem was easily circumvented by employing the swept mesh technique - rather than a structured meshing technique - and specifying the sweep direction or axis along the edges of the section to be modeled using shells. 
Analogous to the cohesive interface elements, high refinement in areas of interest was ensured to capture the localized effects of damage initiation and evolution as a result of the applied pin bearing load.

\subsection{Analysis Steps, Boundary Conditions and Load Case}

The analysis of contact in the pin bearing case represents one of the most challenging modeling scenarios possible when invoking an implicit FE solver. The problem is inherently non-linear as forces change throughout the analysis steps even if they are applied in a quasi-static manner. Rigid body motion must be avoided at all costs since it will lead to numerous solution warnings and errors, which ultimately cause simulation abortion. To account for the above constraints and ensure an accurate simulation without analysis warnings or abortive errors, the applied boundary conditions and load case must be sub-divided into smaller conditions that can be applied carefully over an increased number of analysis steps.

Table 4.10 summarizes the series of applied analysis steps and additional information defined for the analysis. The initial step is not truly an analysis step and is included by default for the establishment of initial boundary conditions and material identification. What should be noted is the use of analyst defined increment sizes rather than simply enabling the commonly employed automatic detection. The reason for this strict enforcement is that automatic detection of these parameters is employed when the solution procedure and the possible occurrences of convergence difficulties is not well known. The software automatically adjusts the step size to be larger of smaller to reflect 
the efficiency of the solution. This requires much computational time and often the autodetection scheme overestimates solution efficiency and increases the step size too much only to cut it back on the next iteration. This ultimately slows down the analysis and can

Table 4.10: Summary of the analysis steps employed, their time periods, consideration of non-linear effects and incrementation.

\begin{tabular}{ccccccc}
\hline Step & $\begin{array}{c}\text { Time } \\
\text { Period } \\
\text { [s] }\end{array}$ & $\begin{array}{c}\text { NL } \\
\text { Geom. }\end{array}$ & $\begin{array}{c}\text { Initial } \\
\text { Inc. } \\
\text { Size [s] }\end{array}$ & $\begin{array}{c}\text { Min. Inc. } \\
\text { Size [s] }\end{array}$ & $\begin{array}{c}\text { Max. Inc. } \\
\text { Size [s] }\end{array}$ & $\begin{array}{c}\text { Max. \# of } \\
\text { Inc. }\end{array}$ \\
\hline Initial & N/A & N/A & N/A & N/A & N/A & N/A \\
\hline Residual & 0.25 & Y & 0.01 & 0.001 & 0.01 & 1000 \\
\hline $\begin{array}{c}\text { LR } \\
\text { Contact }\end{array}$ & 0.25 & $\mathrm{Y}$ & 0.01 & 0.001 & 0.01 & 1000 \\
\hline Contact & 0.25 & $\mathrm{Y}$ & 0.01 & 0.001 & 0.01 & 1000 \\
\hline $\begin{array}{c}\text { Apply } \\
\text { Load }\end{array}$ & 1.0 & $\mathrm{Y}$ & 0.0001 & $1 \times 10^{-6}$ & 0.01 & 1000 \\
\hline
\end{tabular}

be avoided in cases such as the one examined in which the steps that are inherently difficult (contact and applied load) are known to be so and in response a slow, consistent step time is applied to ensure a consistent solution without any cutbacks in incrementation. Closely linked to the analysis steps are the applied boundary conditions which will be created, propagated and modified throughout the steps to ensure a smooth and efficient solution.

Figure 4.7 shows the applied boundary conditions at the initial step of the analysis. The visible boundary conditions include an applied constraint at the far end, a symmetry constraint in the $\mathrm{XZ}$ plane, a displacement constraint on the pin and a displacement constraint on the lateral restraint. The role of each boundary condition within the analysis and subsequent analysis steps is summarized in Table 4.11 and explained below. 
For the analysis at hand, the first step is the initial step designed to create and enforce the necessary boundary conditions to avoid rigid body motion of any of the parts in the assembly.

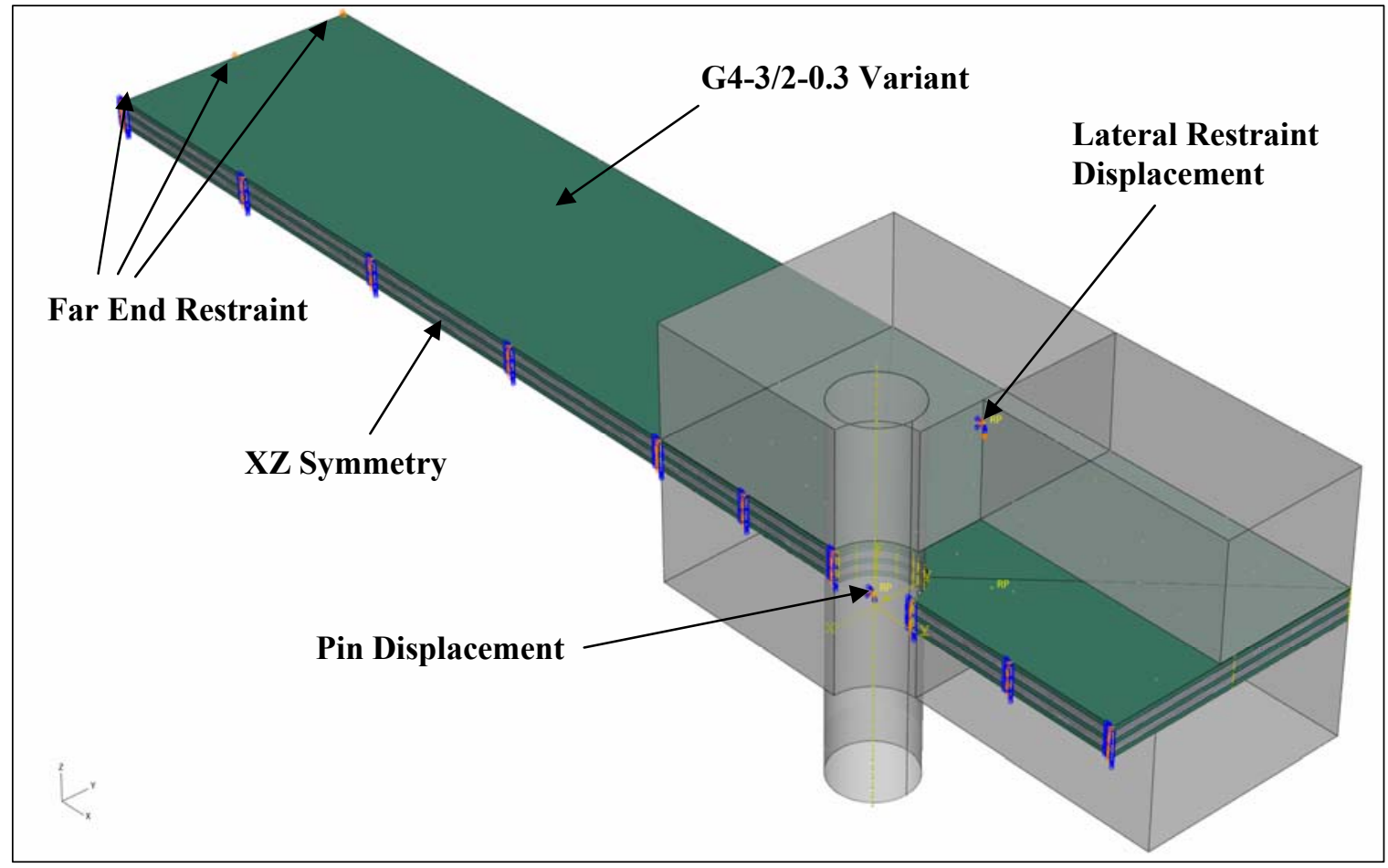

Figure 4.7: Applied boundary conditions on a typical variant analyzed.

Table 4.11: Summary of the applied boundary conditions and their state changes throughout the analysis steps.

\begin{tabular}{cccc}
\hline Analysis Step & Far End $\boldsymbol{B C}$ & Pin $\boldsymbol{B C}$ & Lateral Restraint $\boldsymbol{B C}$ \\
\hline Initial & Created: $\mathrm{U}_{1}=0.0$ & $\begin{array}{c}\text { Created: } \mathrm{U}_{1}=\mathrm{U}_{2}= \\
\mathrm{U}_{3}=\mathrm{UR}_{3}=0.0\end{array}$ & $\begin{array}{c}\text { Created: } \mathrm{U}_{1}=\mathrm{U}_{2}= \\
\mathrm{U}_{3}=\mathrm{UR}_{1}=\mathrm{UR}_{2}= \\
\mathrm{UR}_{3}=0.0\end{array}$ \\
\hline Residual & Propagated & Propagated & Propagated \\
\hline LR Contact & Propagated & Propagated & Modified: $\mathrm{U}_{3}= \pm \varepsilon$ \\
\hline Contact & Propagated & Modified: $\mathrm{U}_{1}=\varepsilon$ & Propagated \\
\hline Apply Load & Propagated & $\begin{array}{c}\text { Modified: } \\
\text { Displacement Freed, } \\
\text { Load Applied }\end{array}$ & Propagated \\
\hline
\end{tabular}


The far end boundary condition applied to the laminate enforces a constraint in the $\mathrm{U}_{1}$ (longitudinal) direction only and fulfills two purposes. The first is to simulate the hydraulic grips analogous to the experimentation which prevented motion along the longitudinal direction. The second purpose is to not enforce a constraint in the remaining two translational degrees of freedom thereby allowing for expansion/contraction of the constituent materials as they undergo the cooling process of the residual stress step. Should enforcement be applied in the $\mathrm{U}_{2}$ and $\mathrm{U}_{3}$ directions, their constraint would generate phantom stresses in the laminate as it cools and will exhibit Poisson's effect during the mechanical loading of the pin in the final step of the analysis.

The pin boundary condition is also created in the initial step and enforces zero displacement in the $\mathrm{U}_{1}, \mathrm{U}_{2}, \mathrm{U}_{3}$ and $\mathrm{UR}_{3}$ directions (here $\mathrm{UR}_{3}$ denotes rotation about the $\mathrm{z}$ axis) to prevent any rigid body motion before contact is established. Relaxation of $\mathrm{UR}_{1}$ and $\mathrm{UR}_{2}$ degrees of freedom is not necessary but is prudent since the incidence of an overconstraint is likely to occur should they not be free to move. In addition, with all translational degrees of freedom constrained, rotational restriction is not necessary for properly constraining the pin.

The applied boundary conditions for the lateral restraint created in the initial step is one of complete restriction of all translational and rotational degrees of freedom to be enforced until contact is established in the LR contact step.

The second step of the analysis is the residual stress step in which the predefined temperature field is applied and a change in temperature generates the aforementioned residual stresses. All boundary conditions for this step are propagated without modification to allow for free expansion/contraction of the constituent materials. 
The third analysis step is the lateral restraint or LR contact step. Here both the far end boundary condition and the pin boundary condition are propagated without modification but now the lateral restraints are moved by a small amount $(\varepsilon)$ until they contact and interact with the laminate surface. The precise amount of this slight adjustment was case specific and dependent upon the amount of contraction that occurred as a result of the residual stress step. Steps of this nature are crucial to any successful contact analysis and are performed under displacement control rather than load control to ensure correct, slow and controlled establishment of contact. Nodes on both the master and slave surfaces must satisfy the contact conditions in a stable manner to prevent rapid and repeated opening and overclosure - a phenomenon referred to as chattering - which will lead to solution abortion in the contact detection step before equilibrium of forces and moments is achieved.

With contact between the lateral restraints and the laminate well established, the next step of the analysis is the enforcement and establishment of contact between the pin and the laminate. In this step, the distal boundary condition and the lateral restraint condition are both propagated without modification and now it is the pin which is moved incrementally forward by a small value $(\varepsilon)$ to establish contact. The previous restriction of motion in the $\mathrm{U}_{2}$ direction is freed and displacement control is used again to ensure clean detection and establishment of contact. The small displacement values, $\varepsilon$, required in both the LR contact step and the contact step unique to each variant analyzed are summarized Table 4.12.

Analogous to the previous analysis step, displacement control is vital in the contact step for clean contact but in addition, if the load was directly applied to the pin in this step 
without first establishing contact or releasing the previous boundary conditions an overconstraint would occur leading to errors and warnings since two distinct conditions would be applied to the same reference point on the pin.

Table 4.12: Summary of the model specific enforced contact displacements applied after the residual stress step.

\begin{tabular}{ccc}
\hline GLARE Variant & $\begin{array}{c}\text { Pin Contact Displacement } \varepsilon \\
\text { [mm] }\end{array}$ & $\begin{array}{c}\text { Lateral Restraint Contact } \\
\text { Displacement } \boldsymbol{\text { [mm }} \text { ] }\end{array}$ \\
\hline GLARE3-5/4-0.3 & 0.0112 & \pm 0.0275 \\
\hline GLARE3-4/3-0.3 & 0.0126 & \pm 0.0250 \\
\hline GLARE4-3/2-0.3 & 0.0102 & \pm 0.0258 \\
\hline
\end{tabular}

Now that contact between both the lateral restraints and the laminate and between the pin and the laminate have been enforced and well established, the mechanical load can be applied to the pin. To accomplish this and to avoid the overconstraint discussed previously, all displacement degrees of freedom for the pin's reference node are released and the load required for yield as defined and taken from the experimental studies presented earlier is applied. The remaining boundary conditions on the other regions of the model are propagated unmodified, at last fully simulating the experimental configuration of an applied pin bearing load.

Finally, to confirm that the applied boundary conditions and load case were enforced correctly, a degree of freedom monitor was created for the reference point on the pin and outputted to the monitor throughout the analysis. If any anomalous displacements or loads were to occur they would appear as jumps in the displacement of the pin throughout 
the analysis steps and time increments. Since this was not the case for any of the variants analyzed it can be concluded that the analysis procedure simulated the experimental setup cleanly and with minimal errors introduced.

\subsection{Requested Field/History Output Variables}

The implementation of contact and progressive damage models requires the requesting of additional field and history output variables. Care must be exercised in requesting these variables since elements not defined by a traction-separation law or elastic-brittle behaviour will not be able to honour the request. A global request for these output variables leads to numerous warnings when running an analysis, which will ultimately slow down the convergence since ABAQUS must check for the request and issue a warning at all successive iterations. To avoid such analysis clutter and indeed produce a simulation which is warning and error free for optimal performance and accuracy, separate element sets were created for each element type and were each issued the appropriate requests rather than applying it to the domain of the global model. Below is a list of the variables requested and their definition which will be further discussed in the next chapter.

Requests in the global domain:

$\mathrm{U}$ - The nodal translations and rotation components and resultant.

$\mathrm{RF}$ - Reaction force components.

CDISP - Displacement components of the surfaces in contact.

CSTRESS - Surface pressure of the surfaces in contact. 
$\underline{\text { Requests for the aluminum elements: }}$

E - The total strain components measured using the logarithmic (true) definition of strain to account for plasticity.

$\mathrm{S}$ - The total (true) stress components and invariants.

PE - The plastic strain components.

PEEQ - The equivalent plastic strain.

$\underline{\text { Requests for the cohesive elements: }}$

$\mathrm{NE}$ - The total strain components measured using the nominal definition of strain. The traction-separation law cannot be defined using logarithmic strain.

$\mathrm{S}$ - The total stress components of which only one normal and two shear stresses are available as per the definition discussed previously.

QUADECRT - This output variable indicates whether or not the quadratic nominal strain damage initiation criterion has been satisfied at a specific material point (integration point) within an element. The values range from 0 to 1 and it is requested under the DMICRT field output variable. This variable is useful for mapping the overall delaminated/damaged area.

SDEG - Overall value of the scalar damage variable D. It represents the scalar stiffness degradation of the element at material/integration points. The values range from 0 to 1 . This variable is also useful for mapping the overall delaminated area.

$\underline{\text { Requests for the elastic-brittle composite elements: }}$

E - The total strain components, again employing logarithmic strain.

$\mathrm{S}-$ The total (true) stress components and invariants. 
DMICRT - All damage initiation criteria components which are useful for comparing different damage initiation modes.

\subsection{Chapter Summary}

In this chapter the methodology for the progressive damage FE models in pin bearing as part of the practical methodology was presented. For the geometry, emphasis was placed on the critical $\mathrm{E} / \mathrm{D}=3$ ratio. For the constituent materials, isotropic strain hardening, mixed-mode delamination, composite failure and thermally induced residual stresses were all implemented. The applied boundary conditions and analysis steps included the curing process and displacement controlled movement of the pin and lateral restraints to ensure a clean, error free analysis that closely modeled the actual pin bearing experiments. The results of the analysis as well as a comparison to the experimentally derived results, scanning electron microscopy of some of the failed specimens and a comparison to analytical calculations will be presented in the next chapter. 


\section{Chapter Five:}

\section{Results and Discussion of the Progressive Damage FE Modeling}

The following is a presentation of the results of both the residual stress step and the applied load step in the progressive damage FE modeling. For the residual stress results, a comparison of the output of both the numerical and analytical investigations to the experimental studies in the literature will be presented. For the applied load results, a comparison of the numerical output to the experimental studies performed herein will also be presented in addition to a direct comparison between the same results and the SEM images taken of some of the failed specimens. A discussion of these results and their relation to the empirical investigations are an important part of the practical damage prediction methodology presented thus far. 


\subsection{Results and Discussion of the Thermally Induced Residual Stresses}

Chapter 4 described in detail the procedure for both the analytical calculation of the thermally induced residual stresses as well as how they were implemented into the progressive damage FE model.

\subsubsection{Results of the Analytical Calculations of Thermally Induced Residual Stress}

Table 5.1 summarizes the results of the analytical calculation of thermally induced residual stress in both the aluminum layers and the glass layers using the aforementioned assumption of isotropy in the glass layers. What should be noted in these results is the fact that regardless of the variant analyzed, the calculations invoking the assumption of isotropy in the glass layers invariably produces the same value for residual stress in the aluminum layers. This is simply a consequence of the definition and simplifying assumption which carries with it no consideration of the laminate lay-up. Furthermore, as anticipated, the resulting residual stresses in the aluminum layers have a tensile sense while the corresponding residual stresses in the glass layers have a compressive one. This result is intuitive once the difference in both the coefficient of thermal expansion for each constituent and the effective cooling rate of each constituent is considered.

Table 5.2 subsequently summarizes the results of the analytical calculation of thermally induced residual stresses in the aluminum layers and both longitudinal and transverse glass layers but now assuming that the glass layers exhibit orthotropic behaviour. 
Table 5.1: Calculated thermally induced residual stresses in both the aluminum and glass layers using the isotropic assumption.

\begin{tabular}{ccc}
\hline GLARE Variant & $\begin{array}{c}\text { Residual Stress in } \\
\text { Aluminum Layers [MPa] }\end{array}$ & $\begin{array}{c}\text { Residual Stress in Glass } \\
\text { Layers [MPa] }\end{array}$ \\
\hline GLARE3-5/4-0.3 & 32.83 & -54.17 \\
\hline GLARE3-4/3-0.3 & 32.83 & -57.78 \\
\hline GLARE4-3/2-0.3 & 32.83 & -39.39 \\
\hline
\end{tabular}

Table 5.2: Calculated thermally induced residual stresses in both the aluminum and glass layers assuming orthotropic glass layers.

\begin{tabular}{cccc}
\hline GLARE Variant & $\begin{array}{c}\text { Residual Stress in } \\
\text { Aluminum Layers } \\
\text { [MPa] }\end{array}$ & $\begin{array}{c}\text { Residual Stress in } \mathbf{0}^{\circ} \\
\text { Glass Layers [MPa] }\end{array}$ & $\begin{array}{c}\text { Residual Stress in } \\
\mathbf{9 0}^{\circ} \text { Glass Layers } \\
\text { [MPa] }\end{array}$ \\
\hline GLARE3-5/4-0.3 & 21.19 & -57.11 & -73.49 \\
\hline GLARE3-4/3-0.3 & 20.26 & -58.28 & -74.76 \\
\hline GLARE4-3/2-0.3 & 20.46 & -50.76 & -65.59 \\
\hline
\end{tabular}

An examination of the results in Table 5.2 immediately shows that the calculated result for the aluminum layers is both variant specific and slightly lower than the results invoking the isotropic assumption. This is due to the fact that with the consideration of directionality in the glass layers, the associated differences in stiffness and coefficient of thermal expansion are now accounted for in the results.

It is also evident that the glass layers in the transverse direction exhibit the largest difference between their calculated compressive residual stress and the accompanying tensile residual stress in the aluminum layers. This difference is by no means nominal and approaches $100 \mathrm{MPa}$ in the case of GLARE3-4/3-0.3. The reason for this larger differential rests on the notion that the associated stiffness and coefficient of thermal expansion for the transverse glass layers is markedly different than that of the aluminum 
layers and thus a greater amount of residual stress will be generated upon termination of the cooling process.

\subsubsection{Results of the FE Simulation of Thermally Induced Residual Stress}

Figure 5.1 through Figure 5.4 depict some of the results of the residual stress analysis step in the FE models. Here we see the Von Mises field output variable measuring residual stress as it developed in the aluminum layers - shown in isolation for clarity - for

all variants analyzed. Note the local geometric effect of the notch and the end edge of the laminate which lead to peak stress values. This result is entirely intuitive since by virtue of compatibility within the laminate, interlaminar stresses would develop as a result of geometric changes and boundary effects in line with the principal orthotropic axis. In addition, we see that though the general distribution of residual stress within the aluminum layers is similar from variant to variant, there are distinct differences. These differences arise because of the actual laminate lay-up in terms of not only the number of longitudinal and transverse prepreg plies but equally importantly which of those two ply types is directly adjacent to the aluminum layer and whether the aluminum is bound on both sides by glass prepreg. Figure 5.1 and Figure 5.2 show the same family of GLARE variant namely GLARE3 but differ in the fact that Figure 5.1 depicts a 5/4 lay-up with a through the thickness symmetrical distribution of glass prepreg layers while Figure 5.2 depicts a $4 / 3$ lay-up where there is a through the thickness bias of transversely oriented glass prepreg plies. The result of this bias is a slight bending of the laminate upward in 


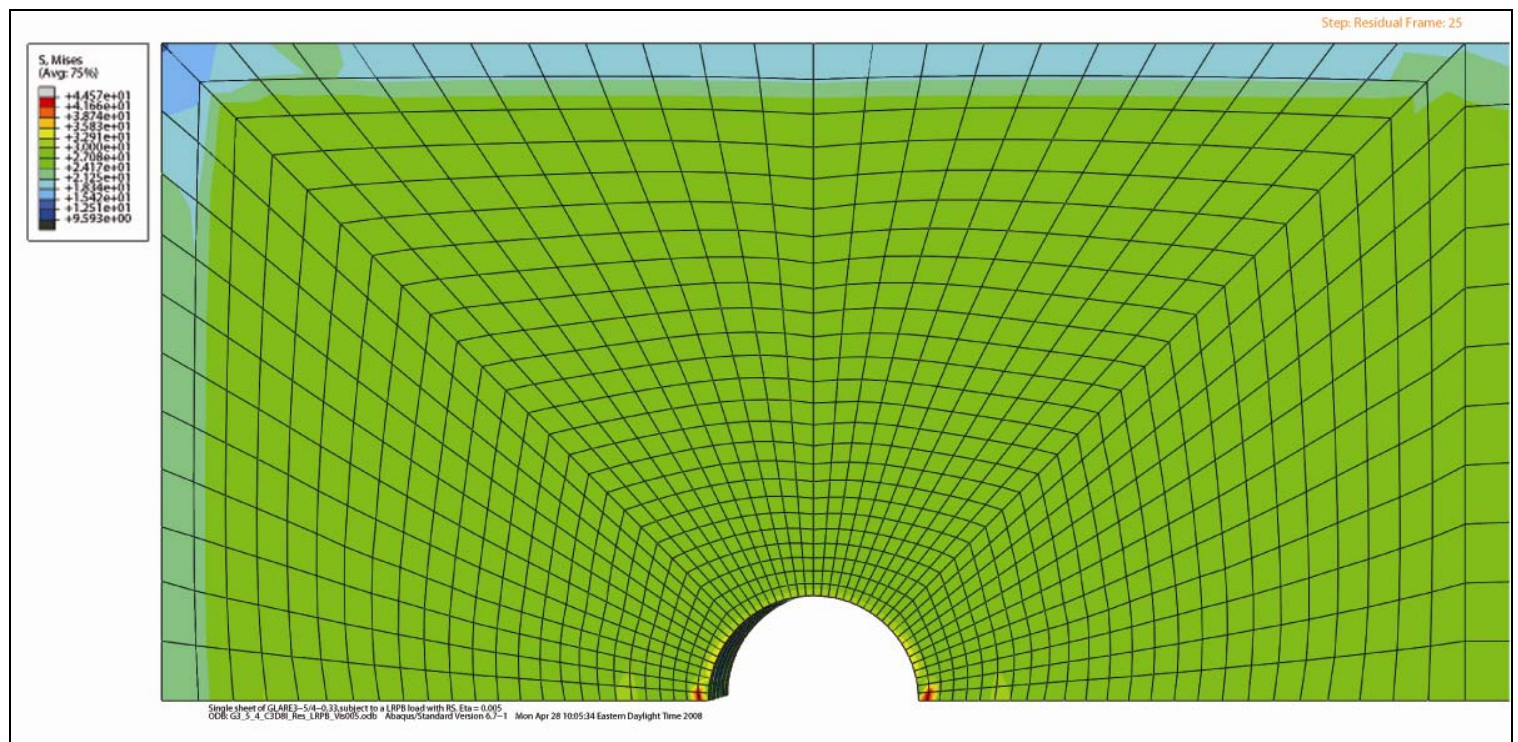

Figure 5.1: Distribution within the aluminum layers of the Von Mises field output variable for the developed residual stresses in a GLARE3-5/4-0.3 variant.

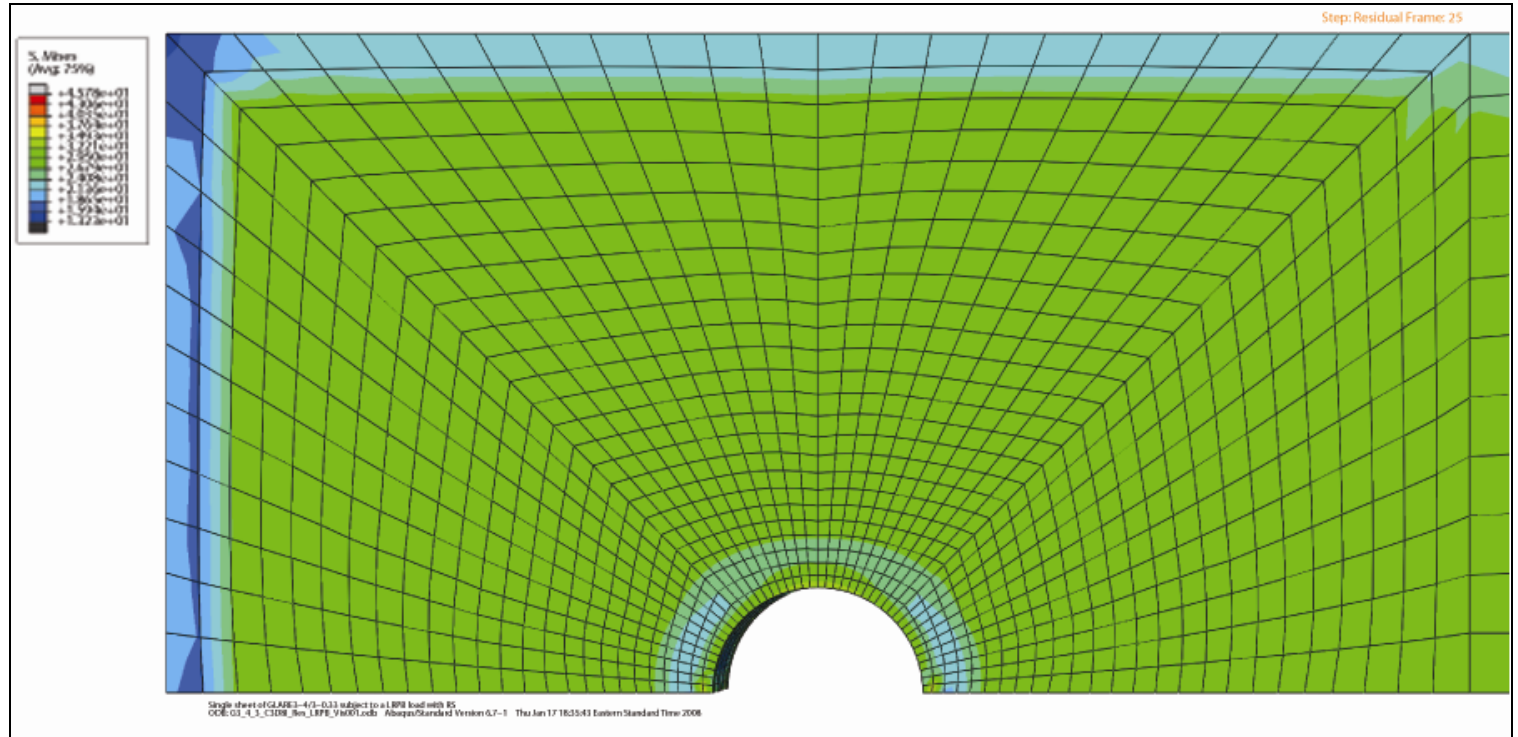

Figure 5.2: Distribution within the aluminum layers of the Von Mises field output variable for the developed residual stresses in a GLARE3-4/3-0.3 variant. 


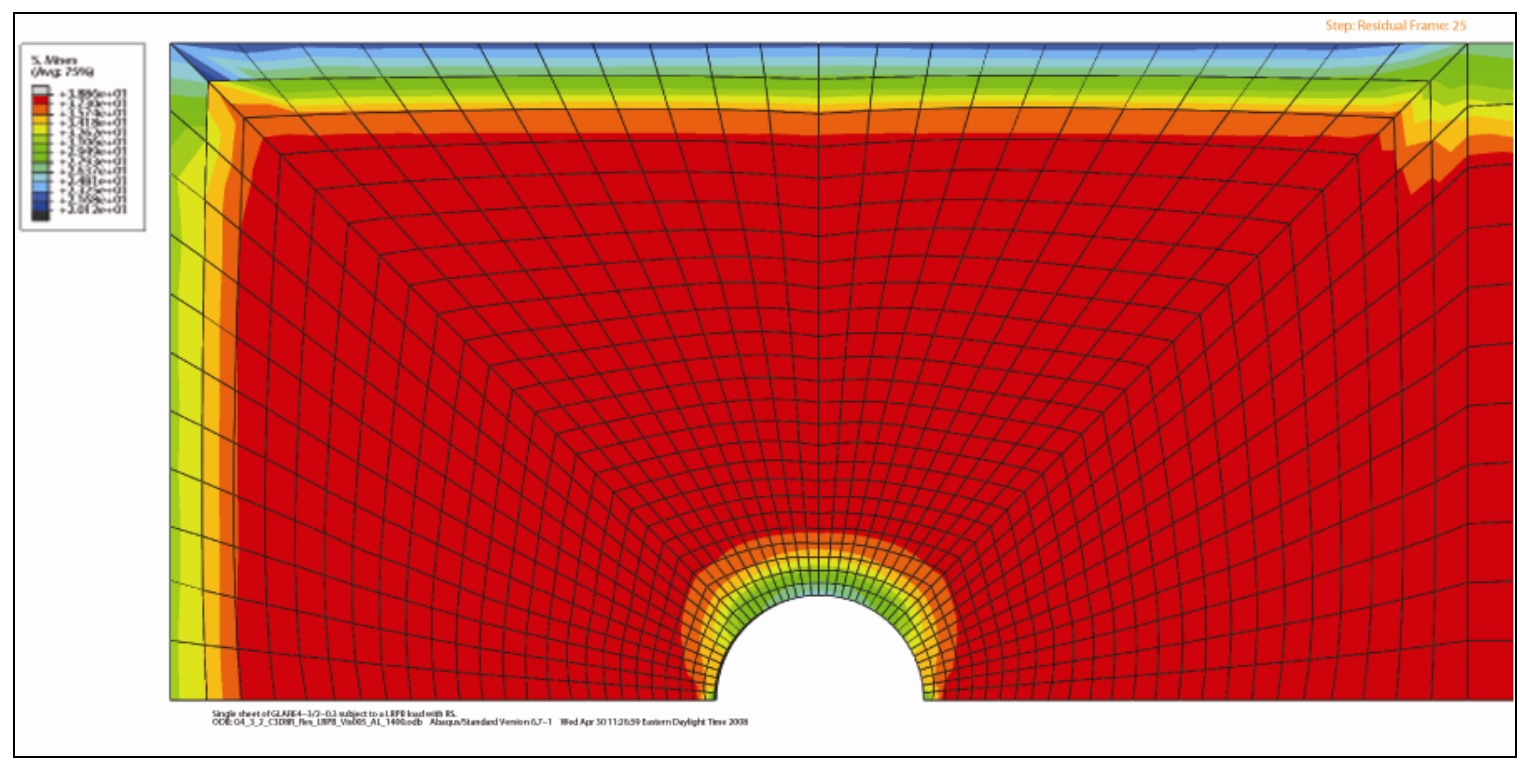

Figure 5.3: Distribution of the Von Mises field output variable within the aluminum layer adjacent to a transverse glass ply bound on one side for the developed residual stresses in a GLARE4-3/2-0.3 variant.

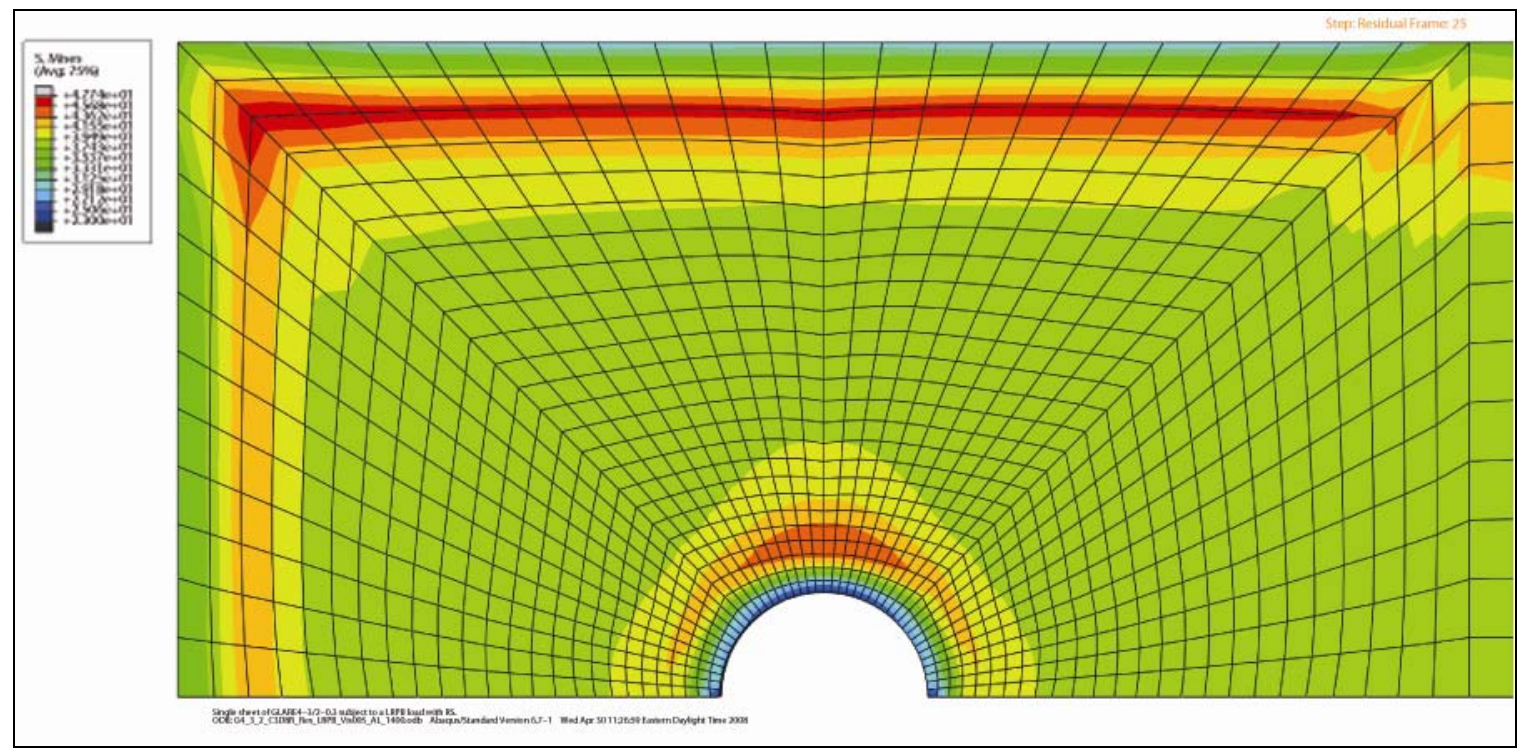

Figure 5.4: Distribution of the Von Mises field output variable within the aluminum layer adjacent to a transverse glass ply bound on both sides for the developed residual stresses in a GLARE4-3/2-0.3 variant. 
the positive z-axis yielding a slightly higher and more pronounced residual stress in and around the pin hole and at the geometric discontinuity of the corner.

With respect to single sided or double sided bounds, Figure 5.3 and Figure 5.4 show this as we see the differences in thermally induced residual stress distribution between a layer of aluminum directly adjacent to a transverse glass layer bound only on one side and a layer of aluminum directly adjacent to a transverse layer bound on both sides. In these cases the aluminum is part of a GLARE4-3/2-0.3 variant where, if we recall, the laminate lay-up consisted of a $90^{\circ} / 0^{\circ} / 90^{\circ}$ configuration for each prepreg layer. Inevitably the outermost aluminum layers will be bound only on one side by the glass layers and thus will develop a different distribution of thermally induced residual stress by virtue of compatibility. Furthermore, aluminum layers bound by both sides will "feel" the influence of the glass layers to a higher degree, particularly the transversely oriented ones since the differential in stiffness and coefficient in thermal expansion is more pronounced.

Figure 5.5 and Figure 5.6 show the distribution of residual stress in a longitudinally oriented glass prepreg layer and a transversely oriented glass layer respectively in a GLARE3-5/4-0.3 variant. Note that the peak stresses generated reveal the bias in stiffness and coefficient of thermal expansion associated with the each layer's principal orthotropic axis. Again this result is entirely intuitive through considerations of the laminate lay-up and its associated directionality.

Figure 5.7 shows the through the thickness distribution of stress in all of the glass layers in the same GLARE3-5/4-0.3 variant. The purpose of including this figure is to illustrate 


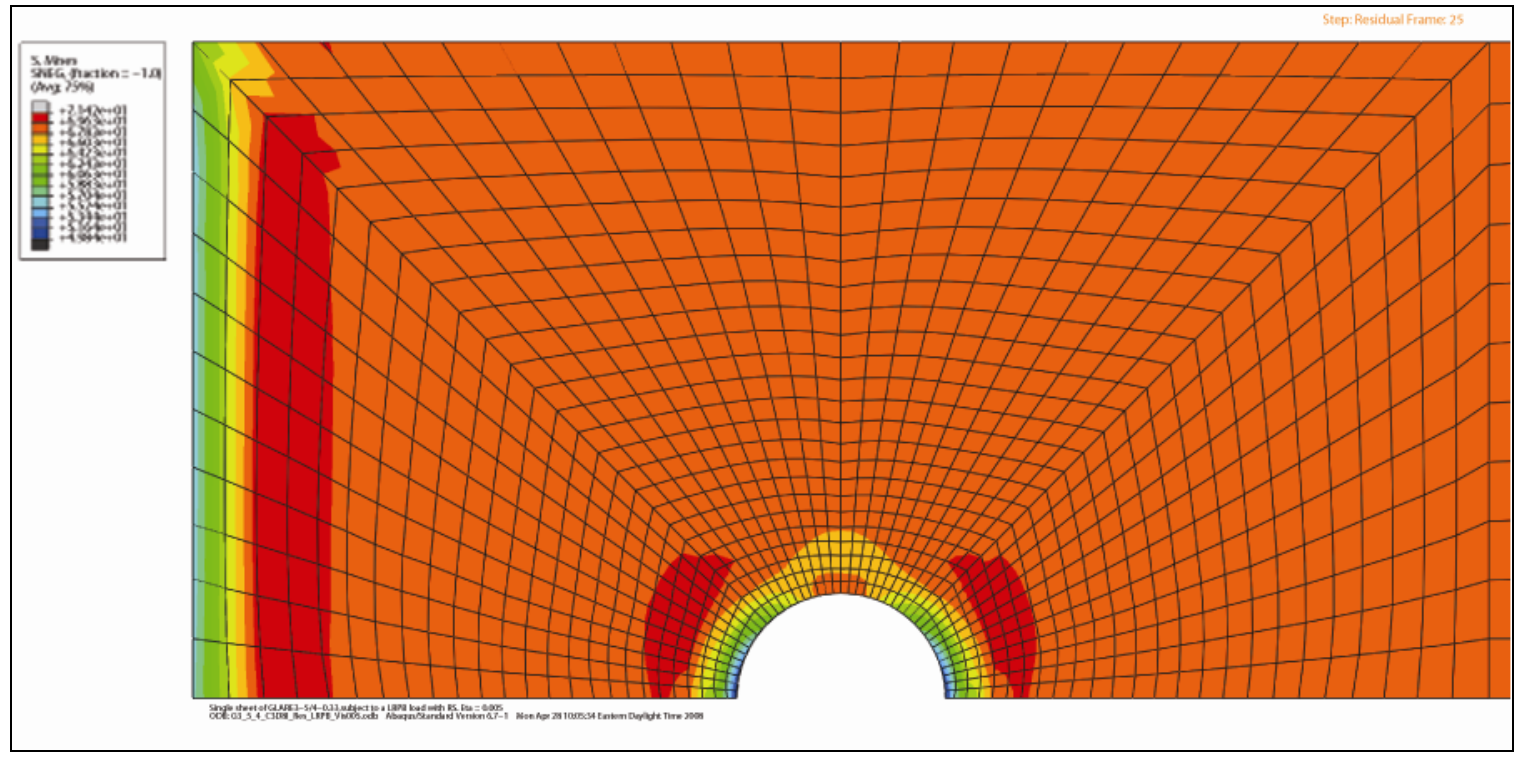

Figure 5.5: Distribution of the Von Mises field output variable for the developed residual stresses in a longitudinally oriented glass prepreg layer in a GLARE3-5/40.3 variant.

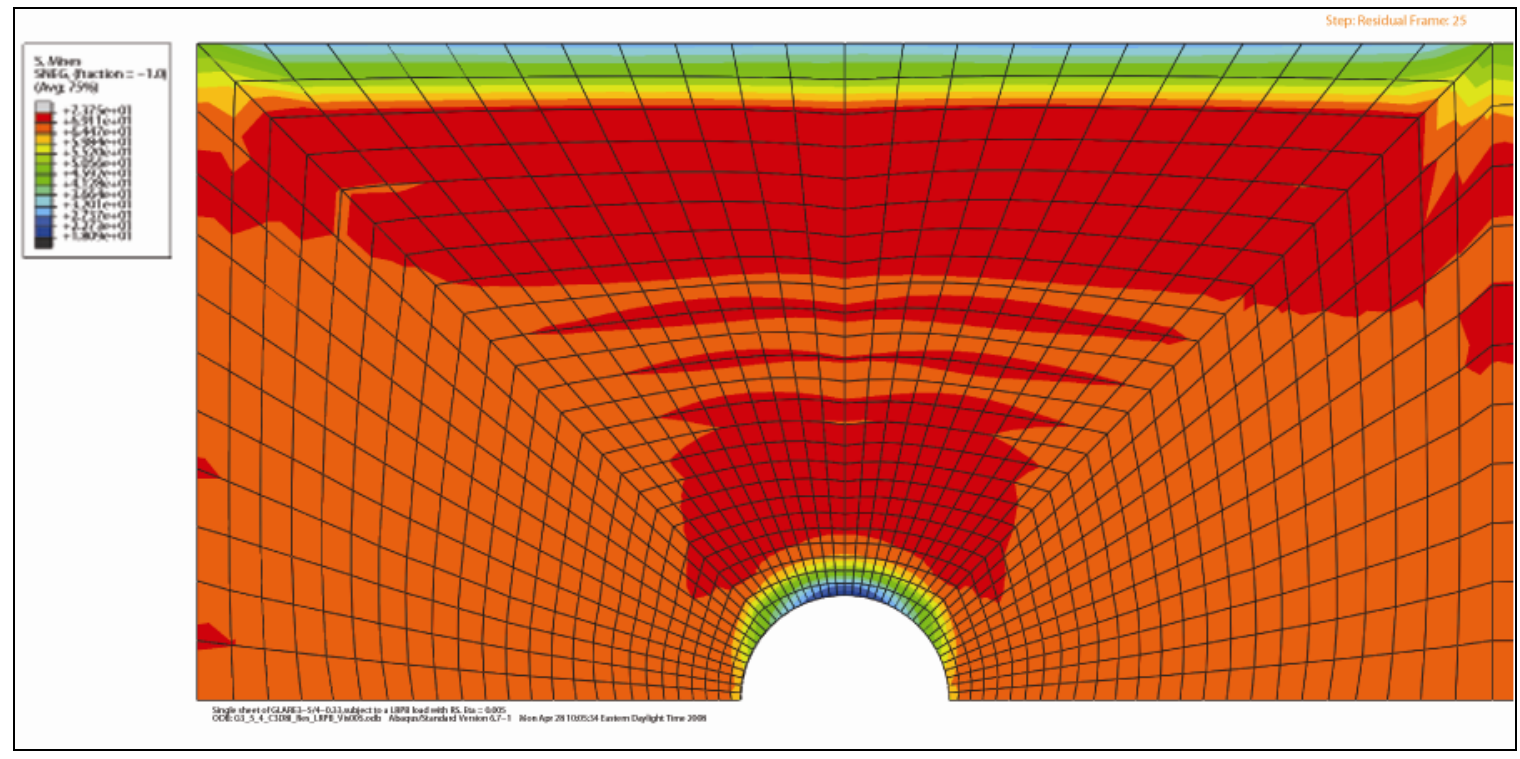

Figure 5.6: Distribution of the Von Mises field output variable for the developed residual stresses in a transversely oriented glass prepreg layer in a GLARE3-5/4-0.3 variant. 


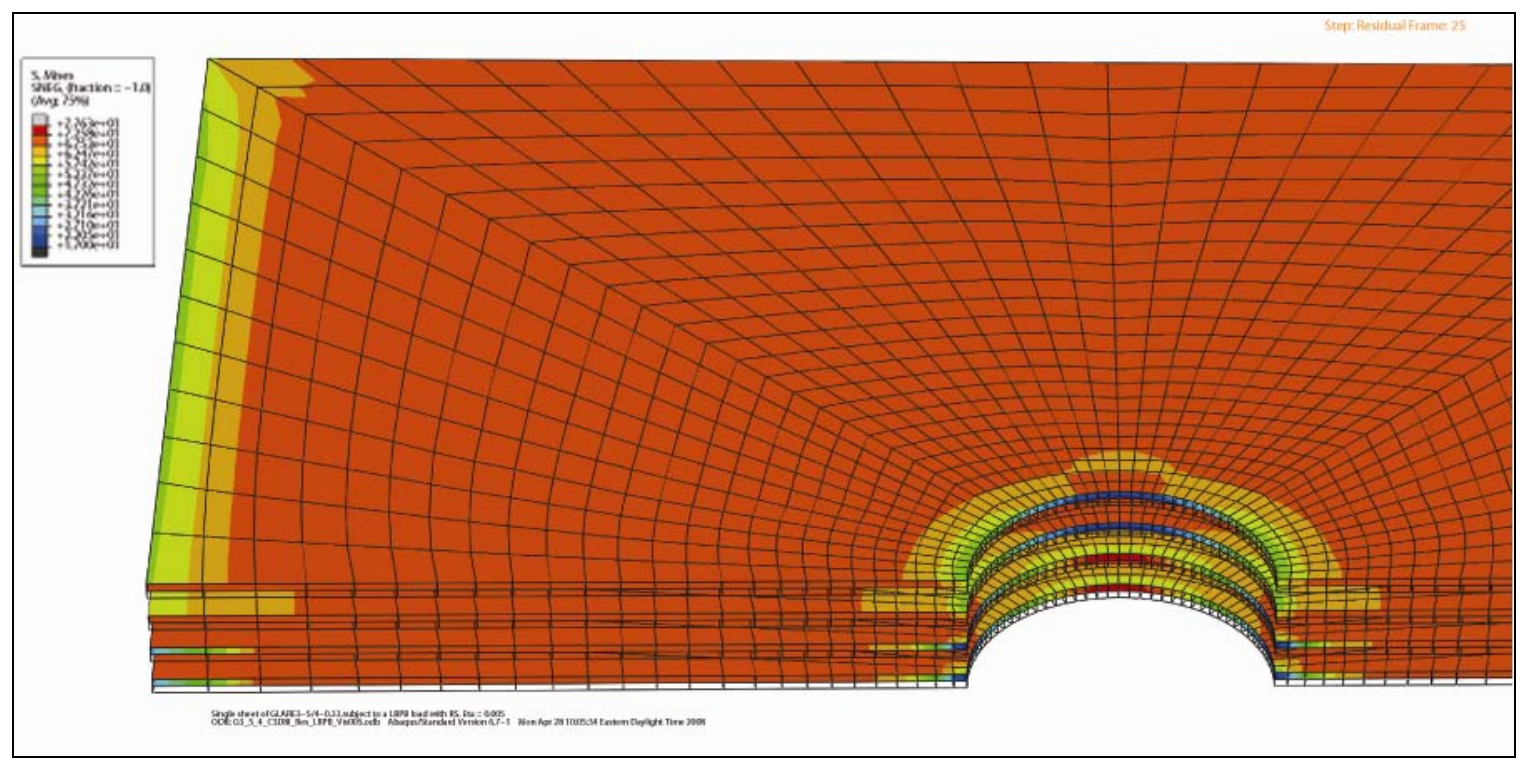

Figure 5.7: Through the thickness distribution of the Von Mises field output variable for the developed residual stresses in all the glass prepreg layers in a GLARE3-5/4-0.3 variant.

that there exists a through the thickness symmetry of peak stresses and general stress distribution for this laminate by virtue of its symmetric lay-up. This symmetry is contrasted markedly in Figure 5.8 where the transverse bias and through the thickness asymmetry of the GLARE3-4/3-0.3 variant is clearly visible in both peak stress and general stress distribution.

Finally, Figure 5.9 through Figure 5.12 show the same plots but now for the GLARE43/2-0.3 variant. The dominance of transversely oriented plies is evident in the distribution of stress but now the same through the thickness symmetry exhibited by the GLARE35/4-0.3 variant is also preserved.

It is also interesting to note the differences in the stress distributions between Figure 5.10 and Figure 5.11. Both of these figures depict the distribution within a transversely oriented ply but differ in that Figure 5.10 is that of a ply directly adjacent to the 


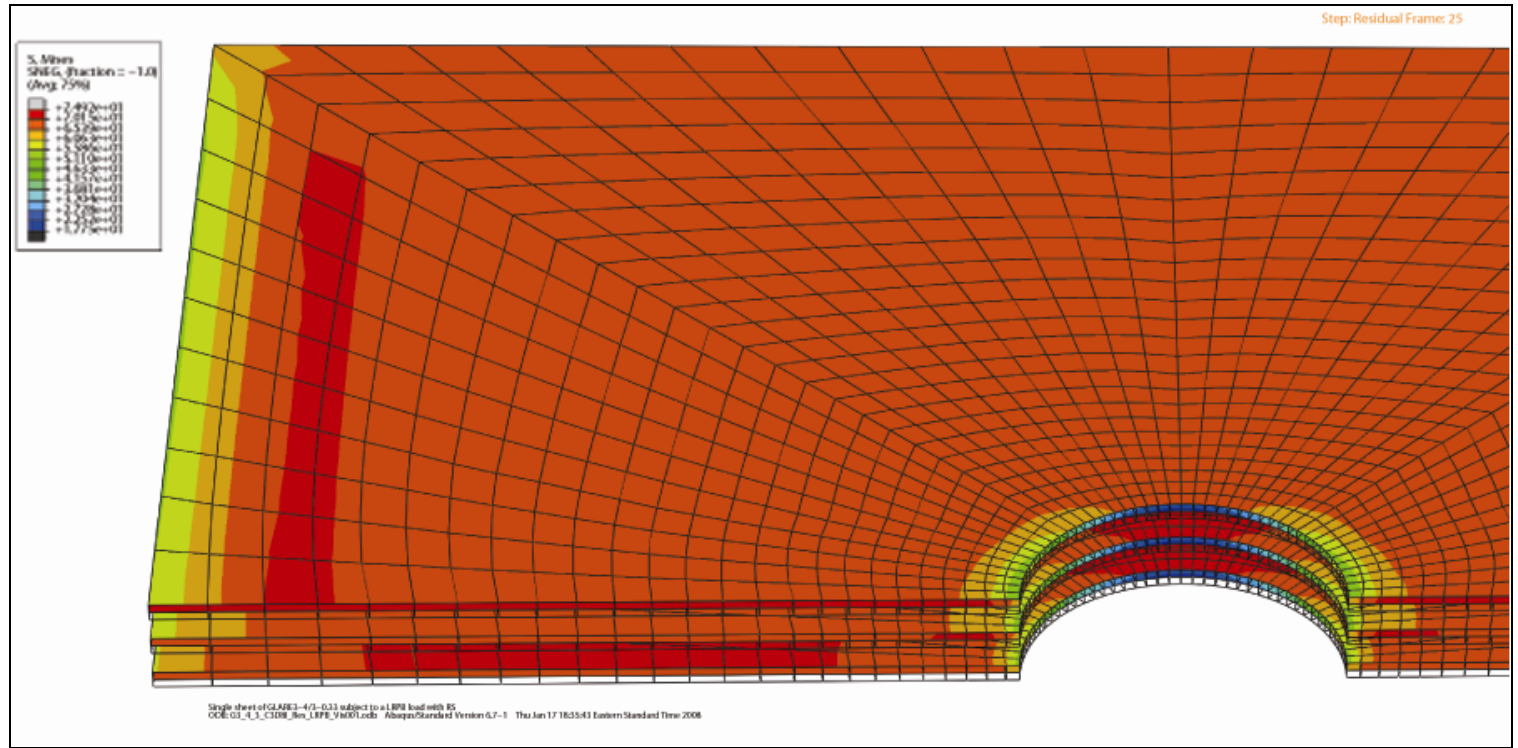

Figure 5.8: Through the thickness distribution of the Von Mises field output variable for the residual stresses in all the glass prepreg layers in a GLARE3-4/3-0.3 variant. Note the asymmetric distribution revealing the transverse bias.

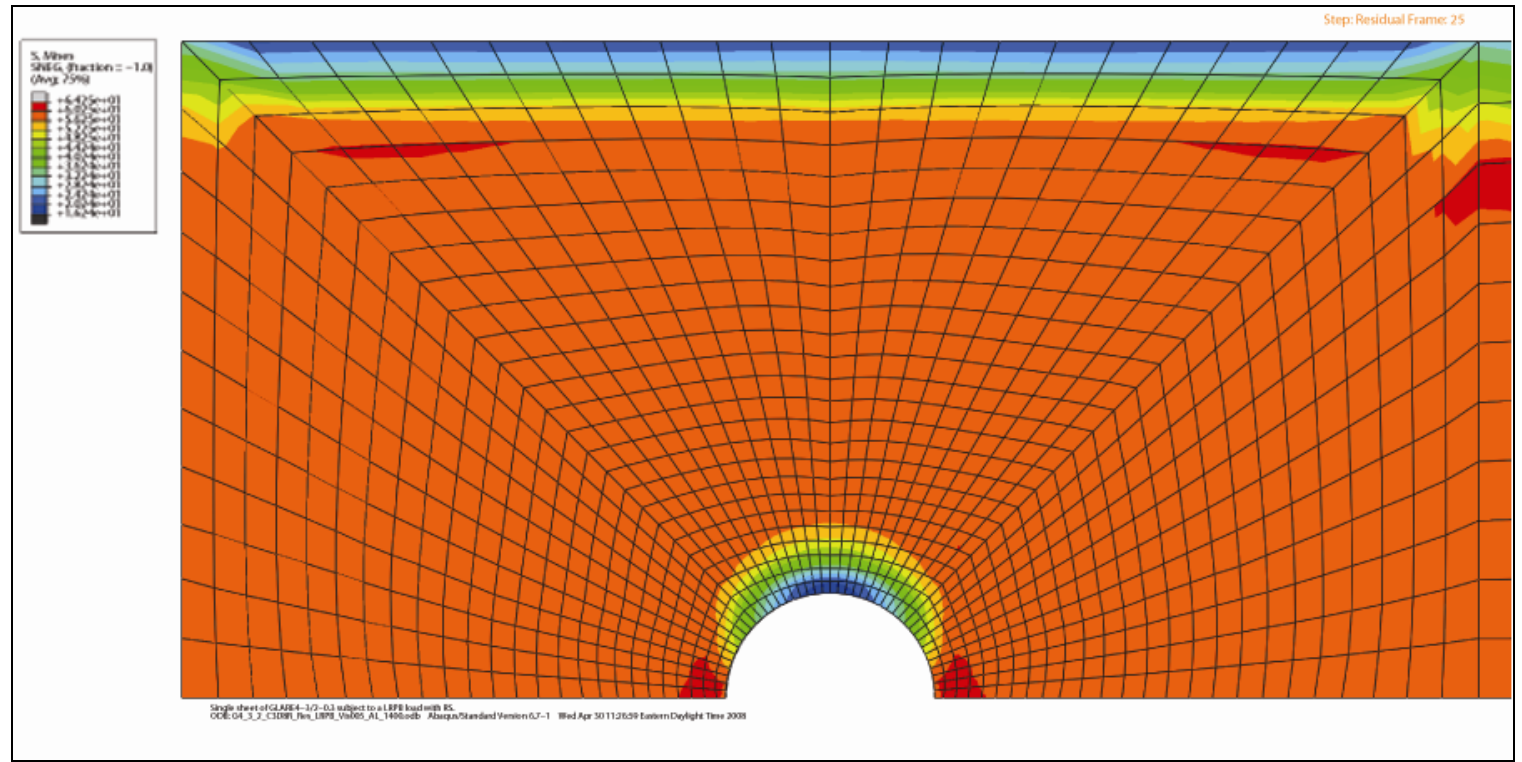

Figure 5.9: Distribution of the Von Mises field output variable for the developed residual stresses in a longitudinally oriented glass prepreg layer in a GLARE4-3/20.3 variant. 


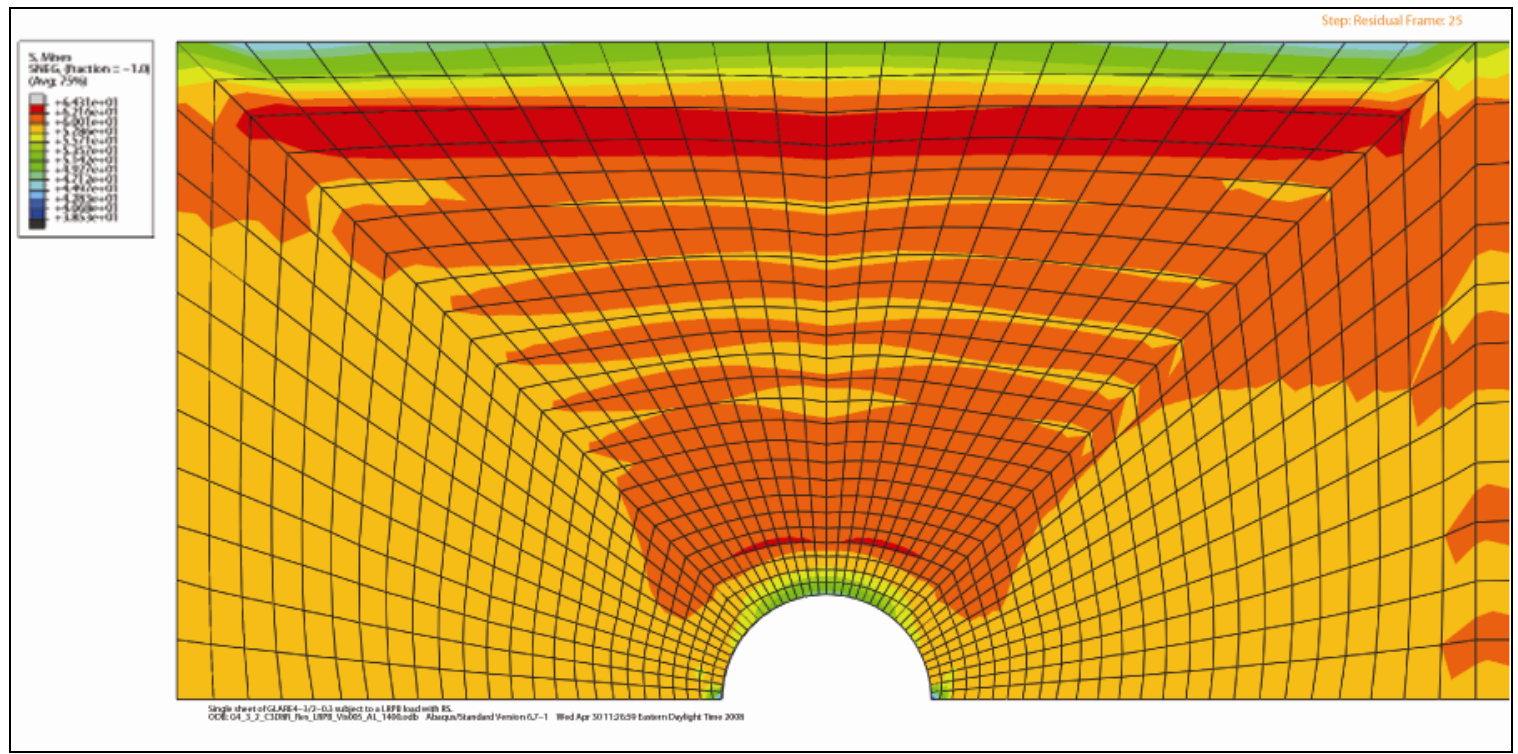

Figure 5.10: Distribution of the Von Mises field output variable for the developed residual stresses in a transversely oriented glass prepreg layer adjacent to the outermost layer of aluminum in a GLARE4-3/2-0.3 variant.

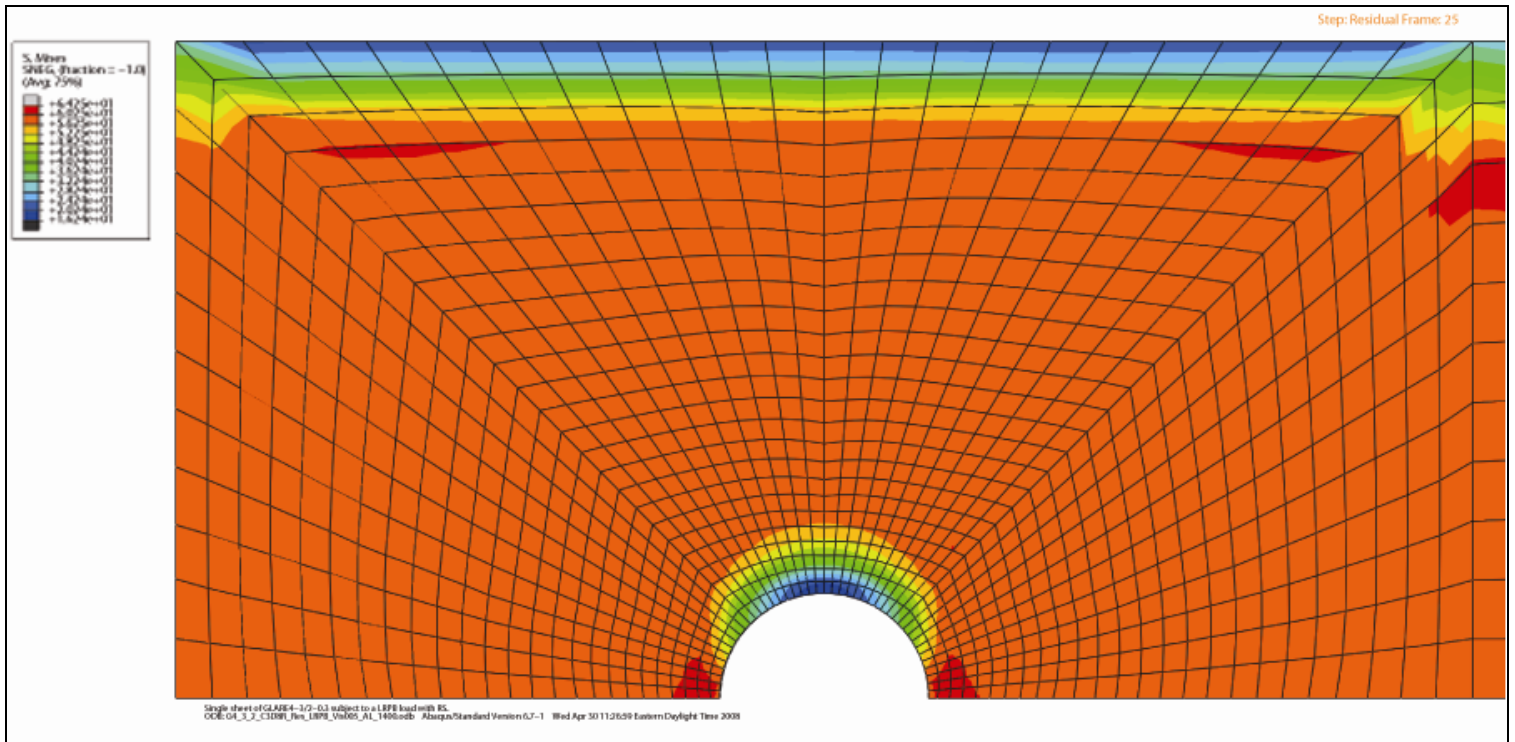

Figure 5.11: Distribution of the Von Mises field output variable for the developed residual stresses in a transversely oriented glass prepreg layer adjacent to the innermost layer of aluminum in a GLARE4-3/2-0.3 variant. 


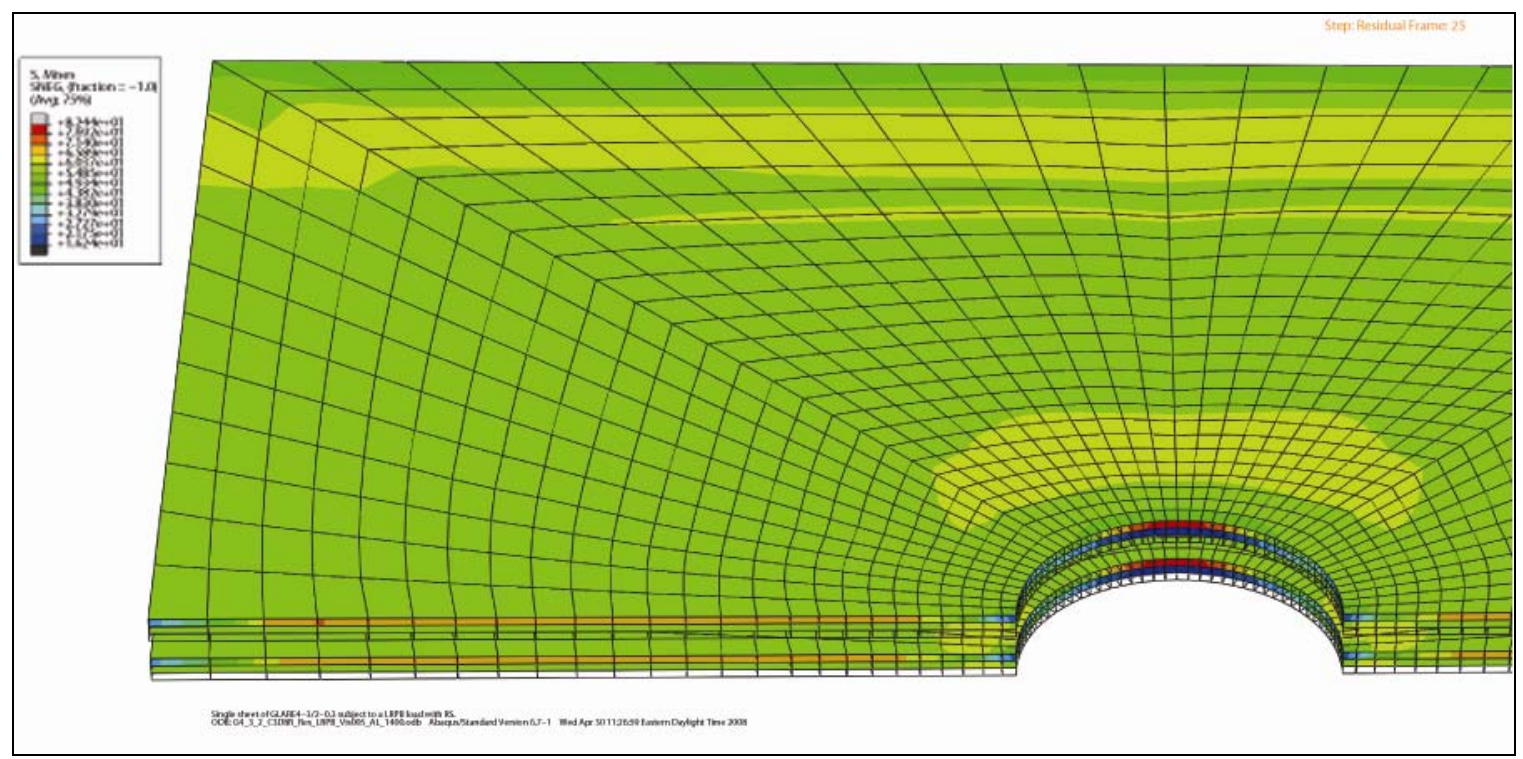

Figure 5.12: Through the thickness distribution of the Von Mises field output variable for the residual stresses in all the glass prepreg layers in a GLARE4-3/2-0.3 variant.

outermost layer of aluminum while Figure 5.11 is that of a ply directly adjacent to the innermost ply of aluminum. Here we see the difference between layers bound on both sides versus binding on a single side. The ply adjacent to the outermost aluminum layer will experience a more pronounced stress since the outermost layer of aluminum is free to contract due to its reduced constraints via its boundary conditions. On the other hand, that same glass layer is bound from the other side by a longitudinally oriented layer of glass prepreg, giving rise to the intricate pattern of stress exhibiting signs of both longitudinal and transverse stress peaks. The other transversely oriented layer in Figure 5.11, which is directly adjacent to the innermost layer of aluminum, is not subject to the same partially free contraction of the aluminum since that aluminum layer is also bound from both sides. The resulting distribution of stress then more closely resembles that displayed by a 
transversely oriented layer within the symmetric GLARE3-4/3-0.3 variant, again an intuitive result.

\subsubsection{Comparison and Discussion of FE Results to Analytical Calculations and the Literature}

For comparison to the analytically derived values, the nominal, far field values of induced residual stress was extracted from the models to avoid the inclusion of geometric effects which are not accounted for in the analytical calculations and create local peaks in the FE models. Table 5.3 shows the nominal, far field values of induced Von Mises stress as extracted from the aluminum layers of the models and compared, alongside the analytical values, to the empirical results of Hoflsagare [123]. Values of induced stress in the aluminum layers were measured in that study via neutron/X-ray diffraction and found the values to be between 25 and $30 \mathrm{MPa}$ [123]. For comparison purposes in this study a mean value of $27.5 \mathrm{MPa}$ was employed.

Table 5.4 summarizes the comparison between analytical values, nominal far field values of induced Von Mises stress as extracted from the glass prepreg layers of the model and the value of $-72 \mathrm{MPa}$ reported by de Vries et al. [86].

An examination of the results tabulated in both Table 5.3 and Table 5.4 reveals that in general, the most consistent approximation of the empirically measured residual stress values is achieved by the FE simulation when compared to the analytical results. For the case of the aluminum values, a range of 25-30 $\mathrm{MPa}$ was reported in the literature and an arbitrary value of $27.5 \mathrm{MPa}$ was chosen as a comparison which is not necessarily the best 
Table 5.3: Comparison of the analytical, FE and literature residual stress results for the aluminum layers.

\begin{tabular}{ccccc}
\hline \multirow{2}{*}{$\begin{array}{c}\text { GLARE Variant } \\
\text { GLARE3- }\end{array}$} & $\begin{array}{c}\boldsymbol{\sigma}_{\boldsymbol{R}} \\
\text { Aluminum } \\
\text { [MPa] }\end{array}$ & $\begin{array}{c}\text { Relative } \\
\text { Error from } \\
\text { FE [\%] }\end{array}$ & $\begin{array}{c}\text { Relative } \\
\text { Error from } \\
\text { [123] [\%] }\end{array}$ \\
\cline { 2 - 5 } 5/4-0.3 & Iso. & 32.83 & 30.83 & 19.38 \\
\cline { 2 - 5 } & Ortho. & 21.19 & 3.76 & 22.95 \\
\hline \multirow{2}{*}{$\begin{array}{c}\text { GLARE3- } \\
\text { 4/3-0.3 }\end{array}$} & FE & 22.71 & - & 17.41 \\
\cline { 2 - 5 } & Ortho. & 20.26 & 12.13 & 26.32 \\
\hline \multirow{2}{*}{$\begin{array}{c}\text { GLARE4- } \\
\text { 3/2-0.3 }\end{array}$} & FE & 22.72 & - & 17.38 \\
\cline { 2 - 5 } & Ortho. & 20.46 & 54.52 & 25.60 \\
\hline
\end{tabular}

Table 5.4: Comparison of the analytical, FE and literature residual stress results for the glass prepreg layers.

\begin{tabular}{|c|c|c|c|c|c|c|c|}
\hline \multicolumn{2}{|c|}{ GLARE Variant } & \multirow{2}{*}{$\begin{array}{l}\sigma_{R} 0^{\circ} \\
\text { Glass } \\
\text { [MPa] } \\
-54.17 \\
\end{array}$} & \multirow{2}{*}{$\begin{array}{c}\text { Relative } \\
\text { Error } \\
\text { from FE } \\
\text { [\%] }\end{array}$} & \multirow{2}{*}{$\begin{array}{c}\text { Relative } \\
\text { Error } \\
\text { from [86] } \\
{[\%]} \\
24.76 \\
\end{array}$} & \multirow{2}{*}{$\begin{array}{l}\sigma_{R} 90^{\circ} \\
\text { Glass } \\
\text { [MPa] } \\
-54.17 \\
\end{array}$} & \multirow{2}{*}{$\begin{array}{c}\text { Relative } \\
\text { Error } \\
\text { from FE } \\
\text { [\%] }\end{array}$} & \multirow{2}{*}{$\begin{array}{c}\begin{array}{c}\text { Relative } \\
\text { Error } \\
\text { from [86] } \\
\text { [\%] }\end{array} \\
24.76 \\
\end{array}$} \\
\hline \multirow{3}{*}{$\begin{array}{c}\text { GLARE3- } \\
5 / 4-0.3\end{array}$} & Iso. & & & & & & \\
\hline & Ortho. & -57.12 & 5.57 & 20.67 & -73.49 & 2.94 & 2.07 \\
\hline & $\mathrm{FE}$ & -60.49 & - & 15.99 & -71.39 & - & 0.85 \\
\hline \multirow{3}{*}{$\begin{array}{c}\text { GLARE3- } \\
4 / 3-0.3\end{array}$} & Iso. & -57.78 & 16.73 & 19.75 & -57.78 & 17.65 & 19.75 \\
\hline & Ortho. & -58.28 & 16.01 & 19.05 & -74.76 & 6.56 & 3.83 \\
\hline & $\mathrm{FE}$ & -69.39 & - & 3.63 & -70.16 & - & 2.56 \\
\hline \multirow{3}{*}{$\begin{array}{c}\text { GLARE4- } \\
3 / 2-0.3\end{array}$} & Iso. & -39.39 & 39.83 & 45.29 & -39.39 & 32.40 & 45.29 \\
\hline & Ortho. & -50.77 & 22.44 & 29.49 & -65.59 & 12.56 & 8.902 \\
\hline & $\mathrm{FE}$ & -65.46 & - & 9.08 & -58.27 & - & 19.06 \\
\hline
\end{tabular}


measure to use as a point for relative error when in fact all the values outputted from the FE results hovered near the reported range.

For the case of the glass prepreg layers, the reported value of - $72 \mathrm{MPa}$ must be taken with a bit of caution since it was not reported to refer to the longitudinal or transverse fibre layers. An examination of the results in Table 5.4 indicates that perhaps it was a report of the highest value of compressive residual stress encountered which was matched with good approximation by the FE model in the transverse layers. The fact that the transverse layers would exhibit the highest residual stress is again intuitive from differential property considerations.

The analytical calculations somewhat underestimate the residual stress values for the GLARE3-4/3-0.3 and GLARE4-3/2-0.3 variants since the formulation does not consider laminate configuration and through the thickness asymmetry, both of which are contributors present in the FE model. Not surprisingly, the analytical formulation invoking isotropy in the glass layers performed rather poorly relative to the other approaches. This result combined with the under-estimative tendency of the orthotropic model further strengthens the case for the FE approach which maintained a high consistency of approximation with good agreement to the empirical results, supporting it as a viable approach to residual stress estimation.

Aside from the calculation and simulation of thermally induced residual stresses in the aluminum and glass prepreg layers, the cohesive interfaces in between each layer of the laminate are also stressed as a result of the laminate cooling process. The induced stress in these resin rich zones arises from the differential contractions experienced by the layers directly adjacent to the cohesive layers, mutually imparting stress on the interface. 
Figure 5.13 and Figure 5.14 depict the induced stresses in the cohesive layers and a through the thickness view for selected GLARE variants.

What is interesting to note here is the same peak stresses originating near geometric discontinuities such as the notch and the edge though in general the distribution of stresses closely mirrors that of the remaining laminate layers.

Finally, Figure 5.15 and Figure 5.16 show the distribution in-plane and through the thickness of the QUADECRT field output variable which was previously mentioned to be the indicator of the satisfaction of the quadratic nominal strain criterion for the onset of delamination damage. Noteworthy here is the undeniable existence of interfacial damage and delamination onset as a result of the residual stress, centred almost exclusively on the notch. This delamination and its effects and coupling to the proposed YDB mechanism will be discussed in greater detail in the next section when the results of the applied load steps in the FE modeling are presented.

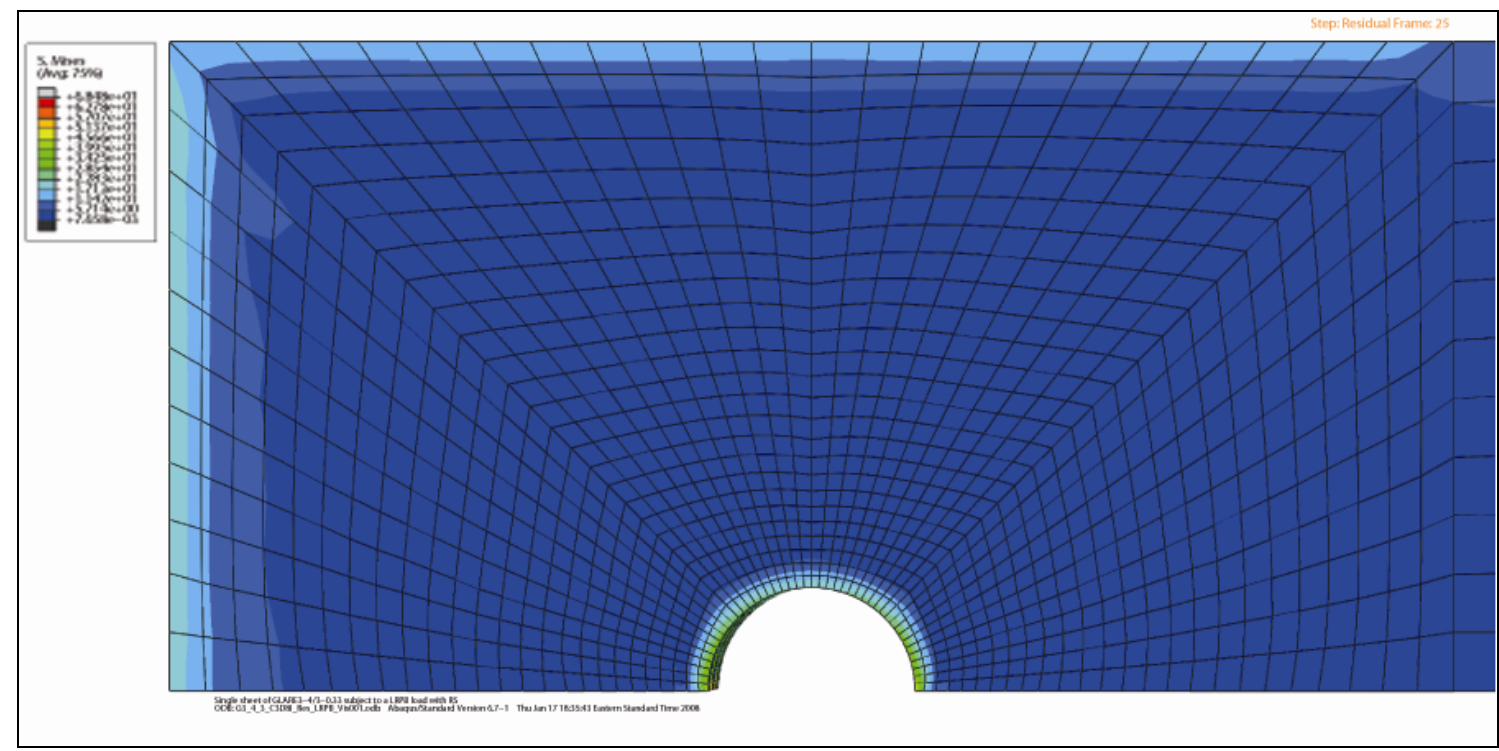

Figure 5.13: Distribution of Von Mises stress in the cohesive layers of a GLARE34/3-0.3 variant. 


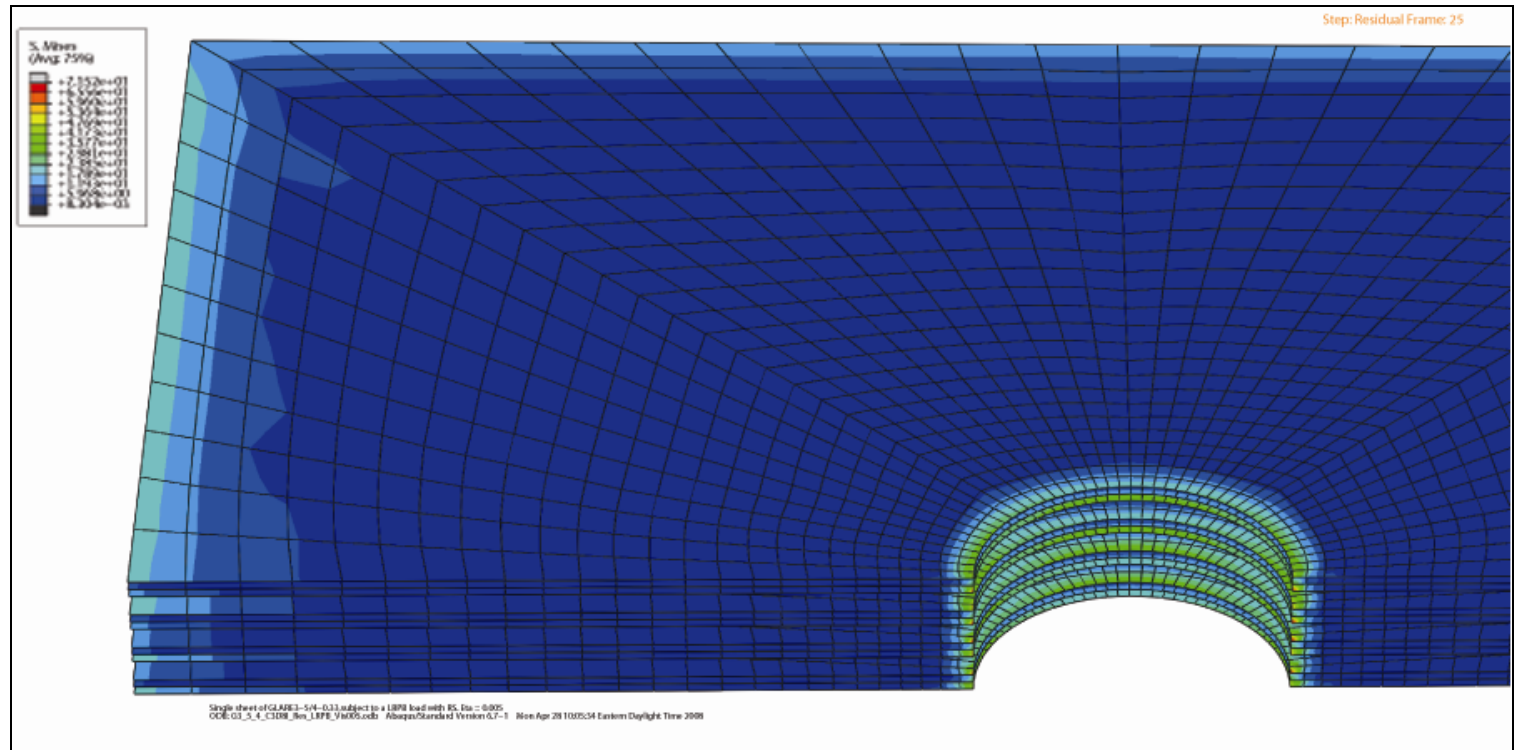

Figure 5.14: Through the thickness distribution of induced Von Mises stress in the cohesive interfaces of a GLARE3-5/4-0.3 variant.

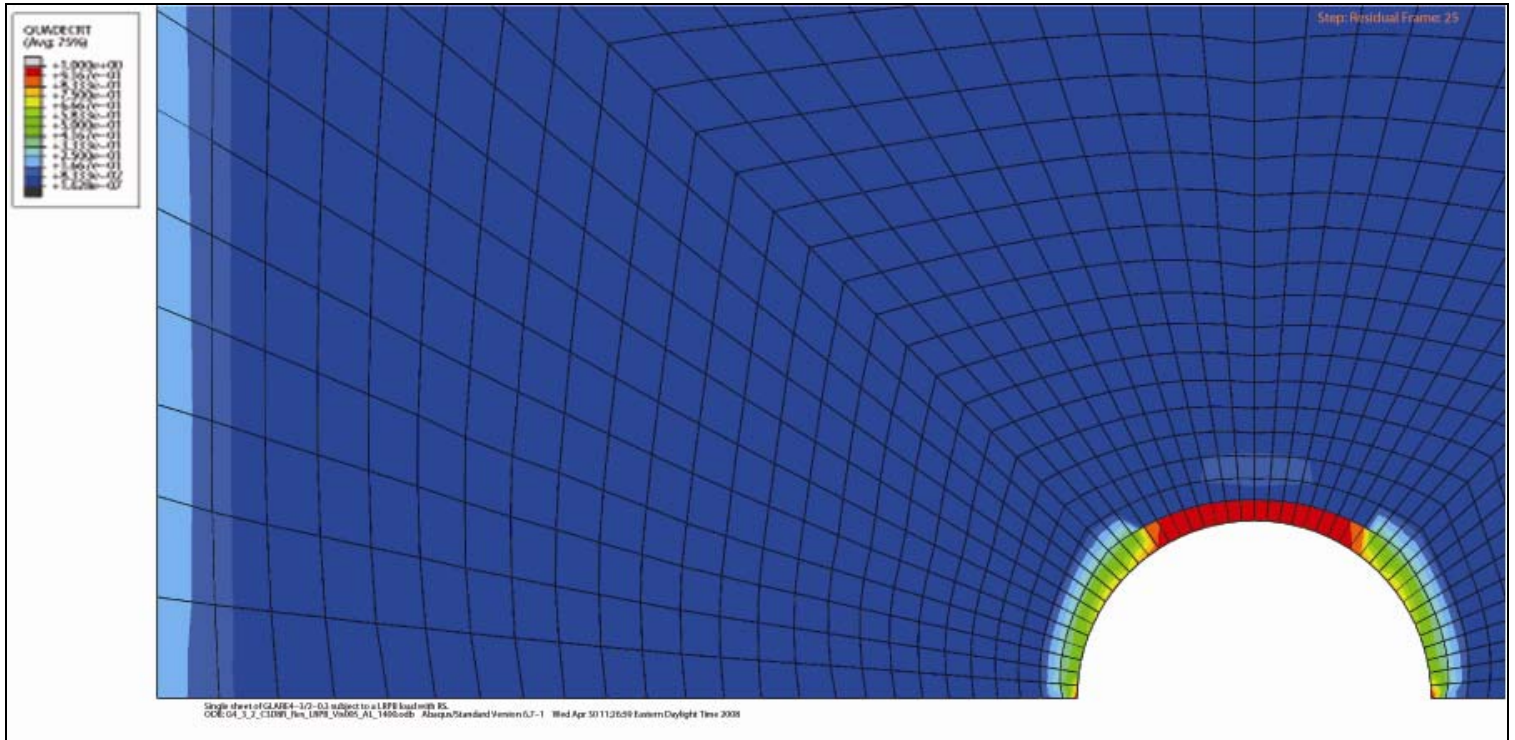

Figure 5.15: Distribution of the QUADECRT field output variable indicating the onset of delamination due to residual stress induction in a cohesive layer within a GLARE4-3/2-0.3 variant. 


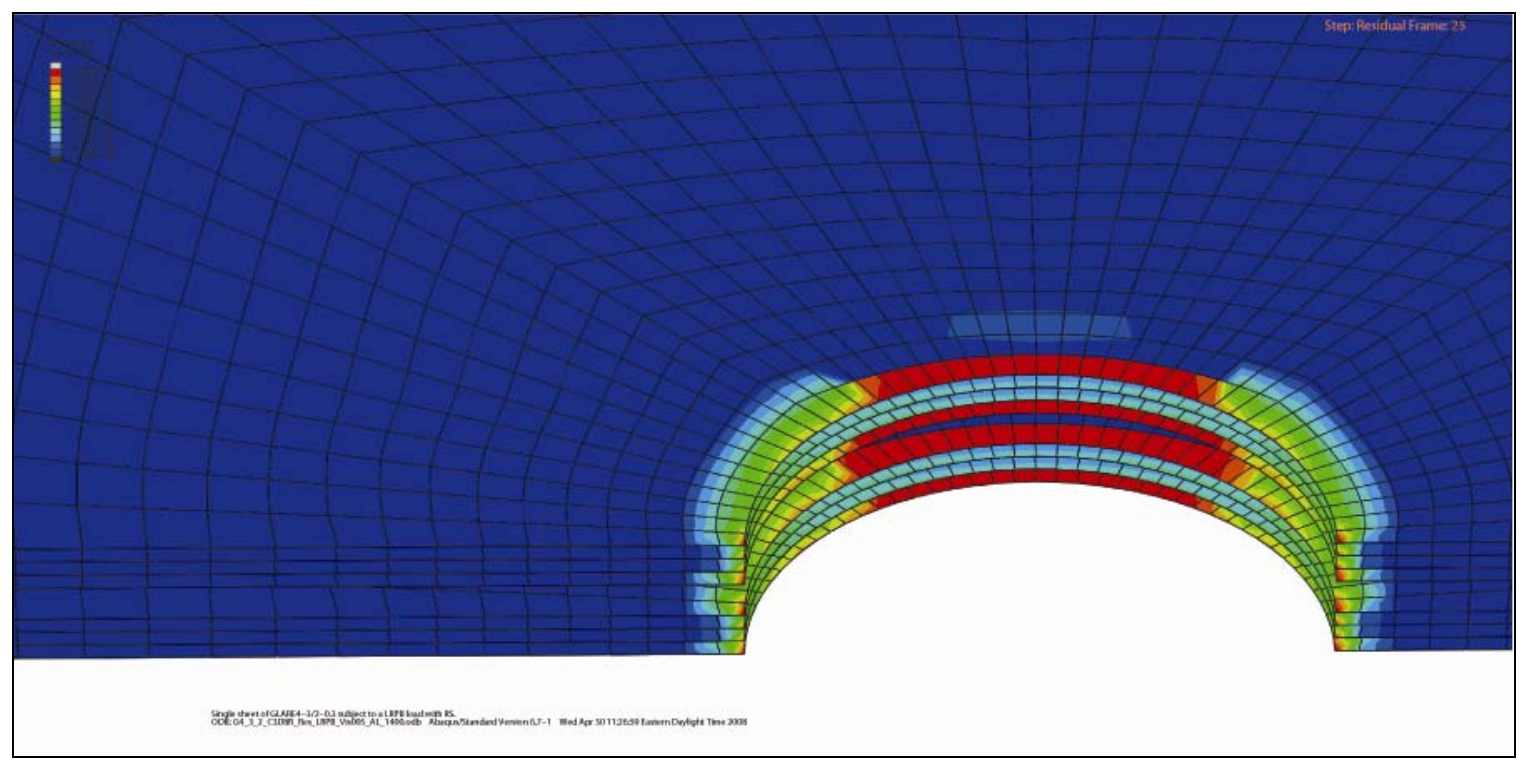

Figure 5.16: Through the thickness distribution of the QUADECRT field output variable as a result of induced residual stresses in a GLARE4-3/2-0.3 variant. Note that damage to the interface, though slight, has already occurred.

\subsection{Results and Discussion of the Applied Loading in the Progressive Damage FE Modeling}

With the residual stress state in the laminate induced through the predefined temperature

field in the residual stress step and subsequent contact steps establishing solid contact between the laminate and pin/lateral restraints, the applied load step followed suit.

\subsubsection{Results of the Applied Load Step}

Figure 5.17 through Figure 5.19 show the calculated contact pressure between the pin and the aluminum layers shown in isolation at the end of the applied load step for all the variants analyzed. 


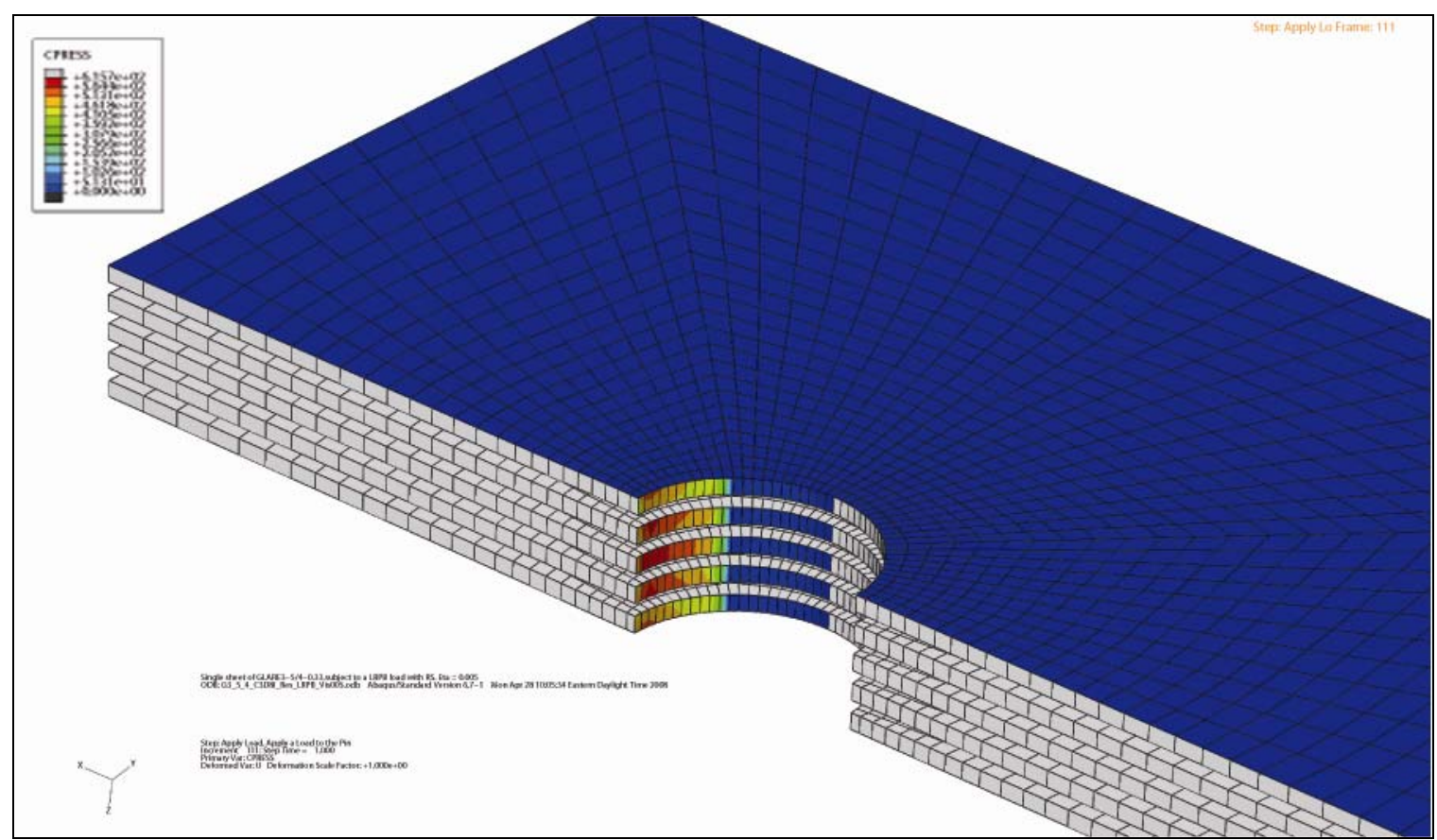

Figure 5.17: Calculated contact pressure between the pin and the laminate at the end of the applied load step for a GLARE3-5/4-0.3 laminate.

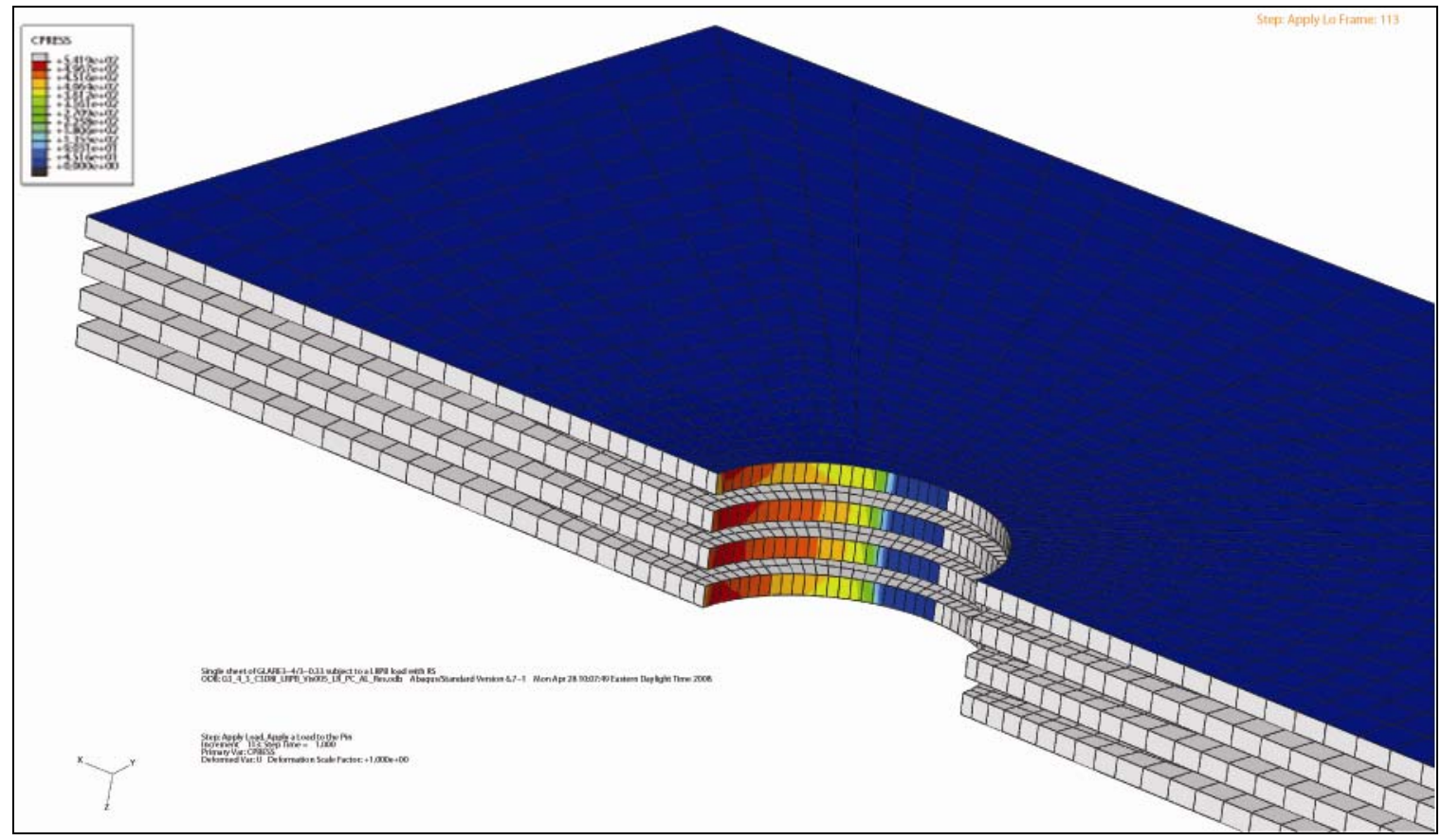

Figure 5.18: Calculated contact pressure between the pin and the laminate at the end of the applied load step for a GLARE3-4/3-0.3 laminate. 


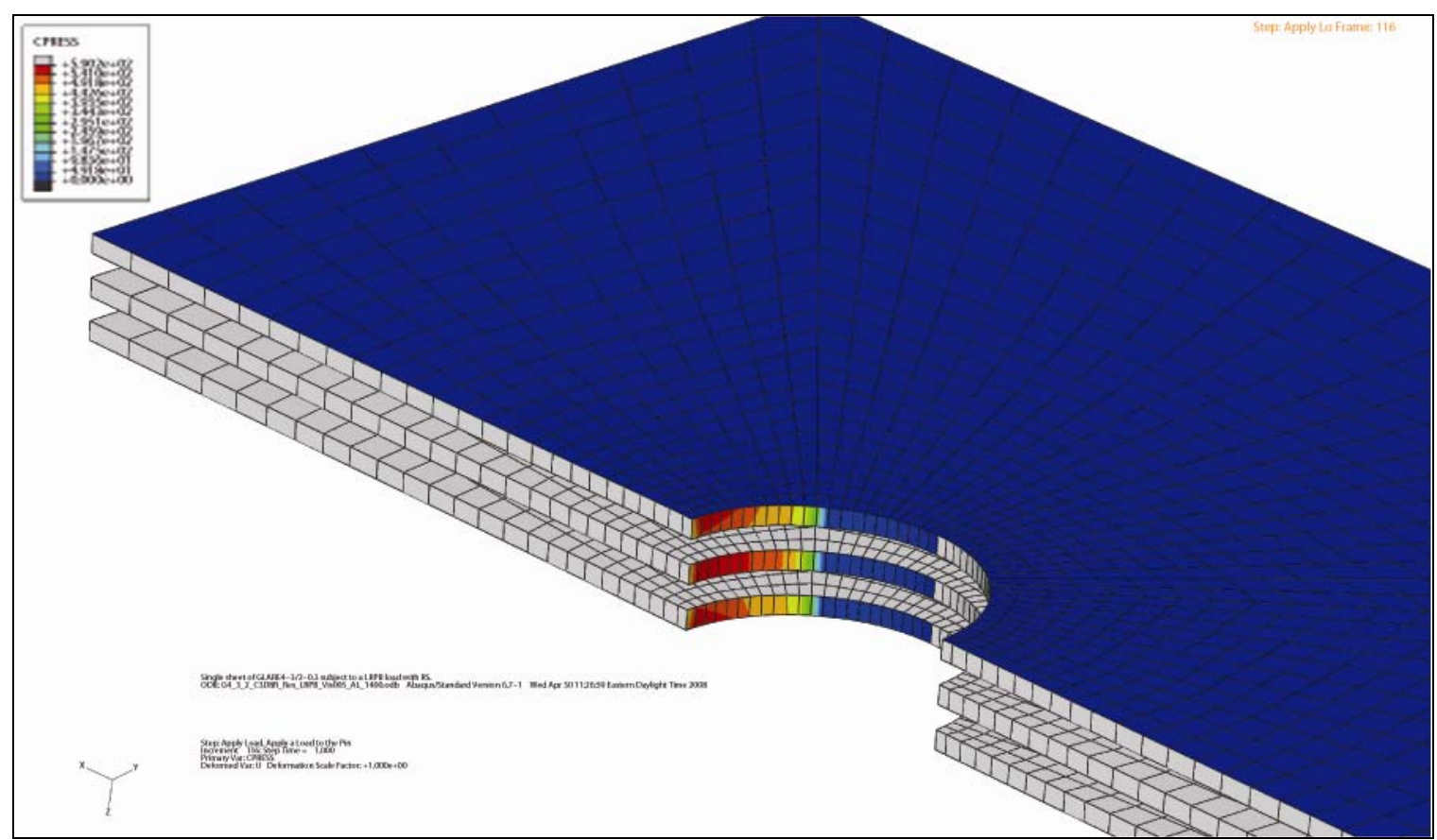

Figure 5.19: Calculated contact pressure between the pin and the laminate at the end of the applied load step for a GLARE4-3/2-0.3 laminate.

Note the location of maximum contact pressure which corresponds to the edge from which the measurement of compressive stresses moving away from the fastener hole begins. These figures illustrate that good contact between the pin and laminate was established as well as the contact induced between the lateral restraints and the laminate, though with a much smaller magnitude.

Table 5.5 summarizes the loads applied to the half symmetry progressive damage FE model rounded to the nearest whole number as taken from the pin bearing experimental study discussed earlier, according to the bisection protocol definition of bearing yield. The remaining figures depicting FE results in this chapter represent the induced strains, stresses and damage distributions - among others - as a result of these applied loads. 
Table 5.5: Summary of the loads applied to the progressive damage FE models as taken from the experimental study and bisection protocol yield definition.

\begin{tabular}{ccc}
\hline GLARE Variant & Bearing Load at Yield [N] & $\begin{array}{c}\text { Bearing Load Applied to FE } \\
\text { Models [N] }\end{array}$ \\
\hline GLARE3-5/4-0.3 & 4168.77 & 2085.0 \\
\hline GLARE3-4/3-0.3 & 3593.50 & 1800.0 \\
\hline GLARE4-3/2-0.3 & 3247.75 & 1620.0 \\
\hline
\end{tabular}

\subsubsection{Results and Discussion for the Aluminum Layers}

Figure 5.20 through Figure 5.26 depict both the Von Mises stress distribution and the logarithmic strain distribution in the aluminum layers for all the variants analyzed. The inclusion of the distribution of logarithmic strain in the longitudinal direction is

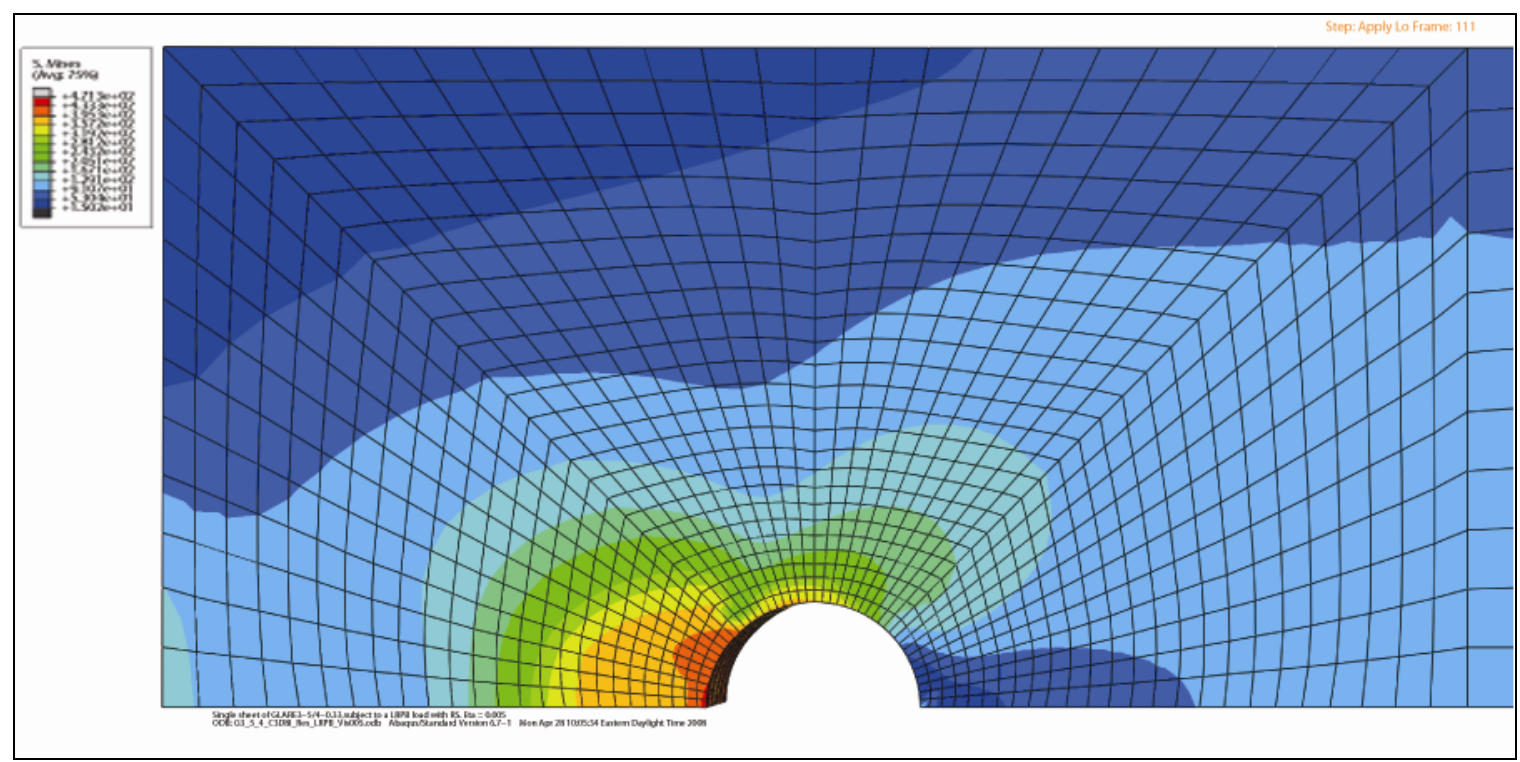

Figure 5.20: Distribution of the Von Mises stress field output variable in the aluminum layers resulting from the applied pin bearing load in a GLARE3-5/4-0.3 laminate. 


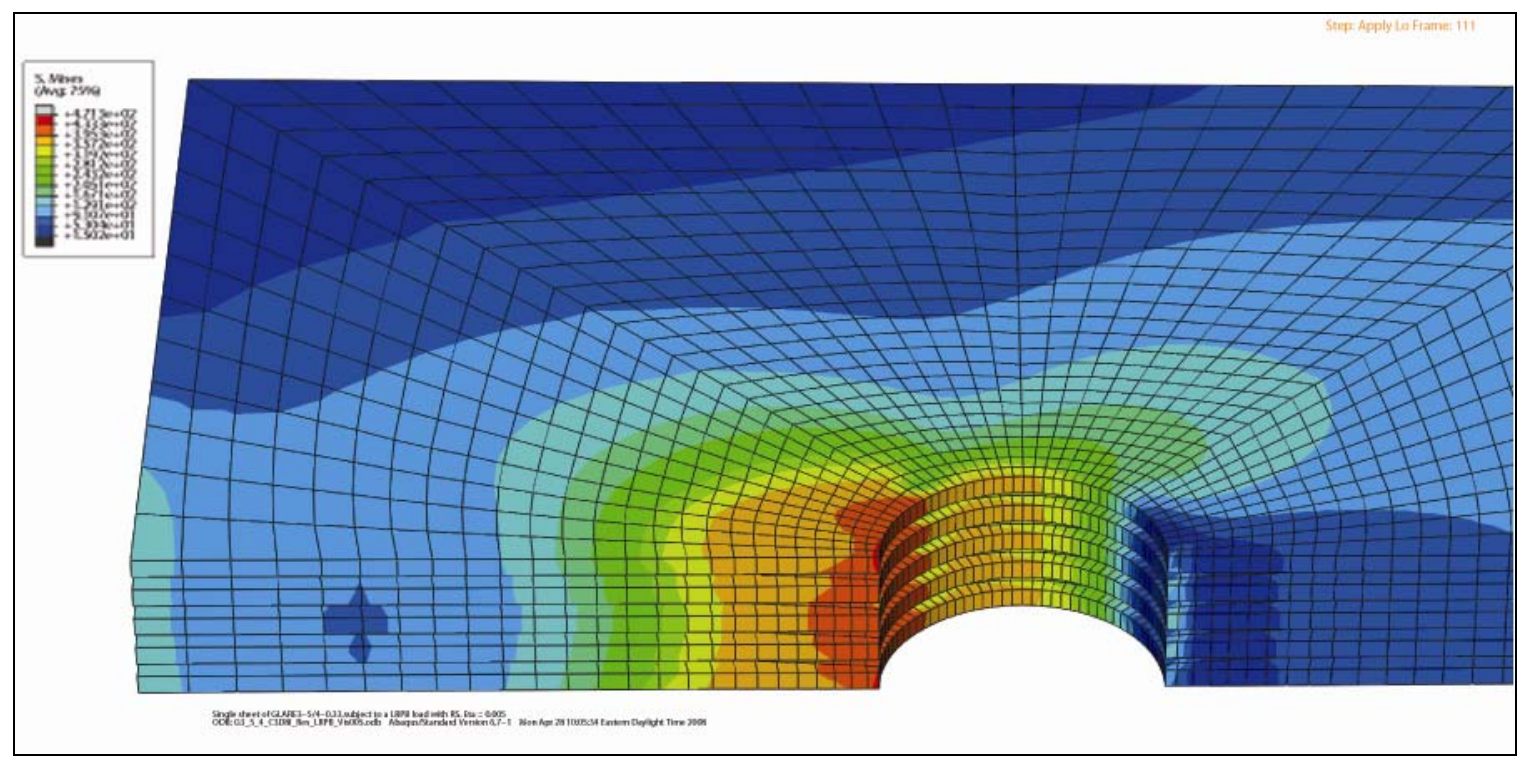

Figure 5.21: Through the thickness distribution of the Von Mises stress field output variable in the aluminum layers resulting from the applied pin bearing load in a GLARE3-5/4-0.3 laminate.

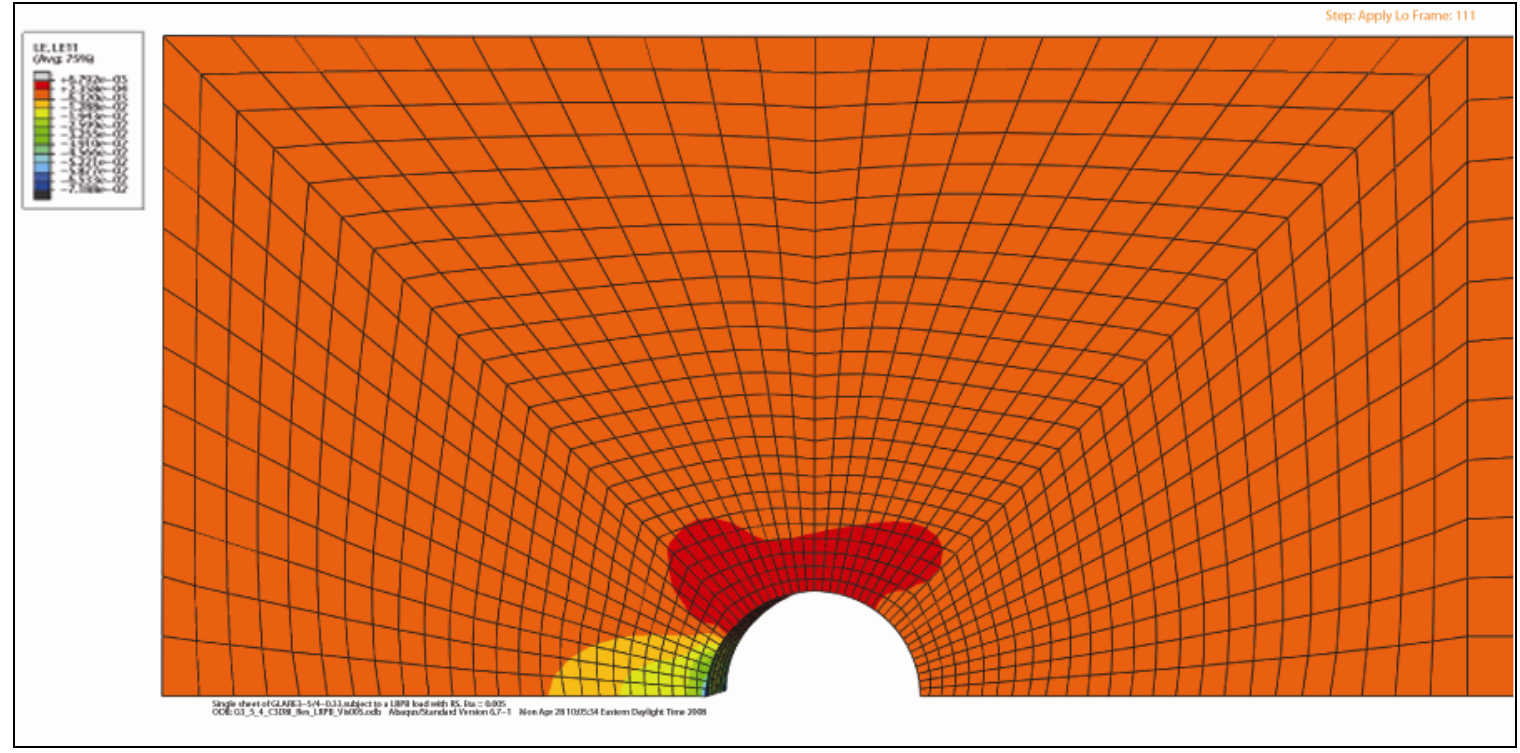

Figure 5.22: Distribution of the logarithmic strain in the longitudinal direction field output variable in the aluminum layers resulting from the applied pin bearing load in a GLARE3-5/4-0.3 laminate. 


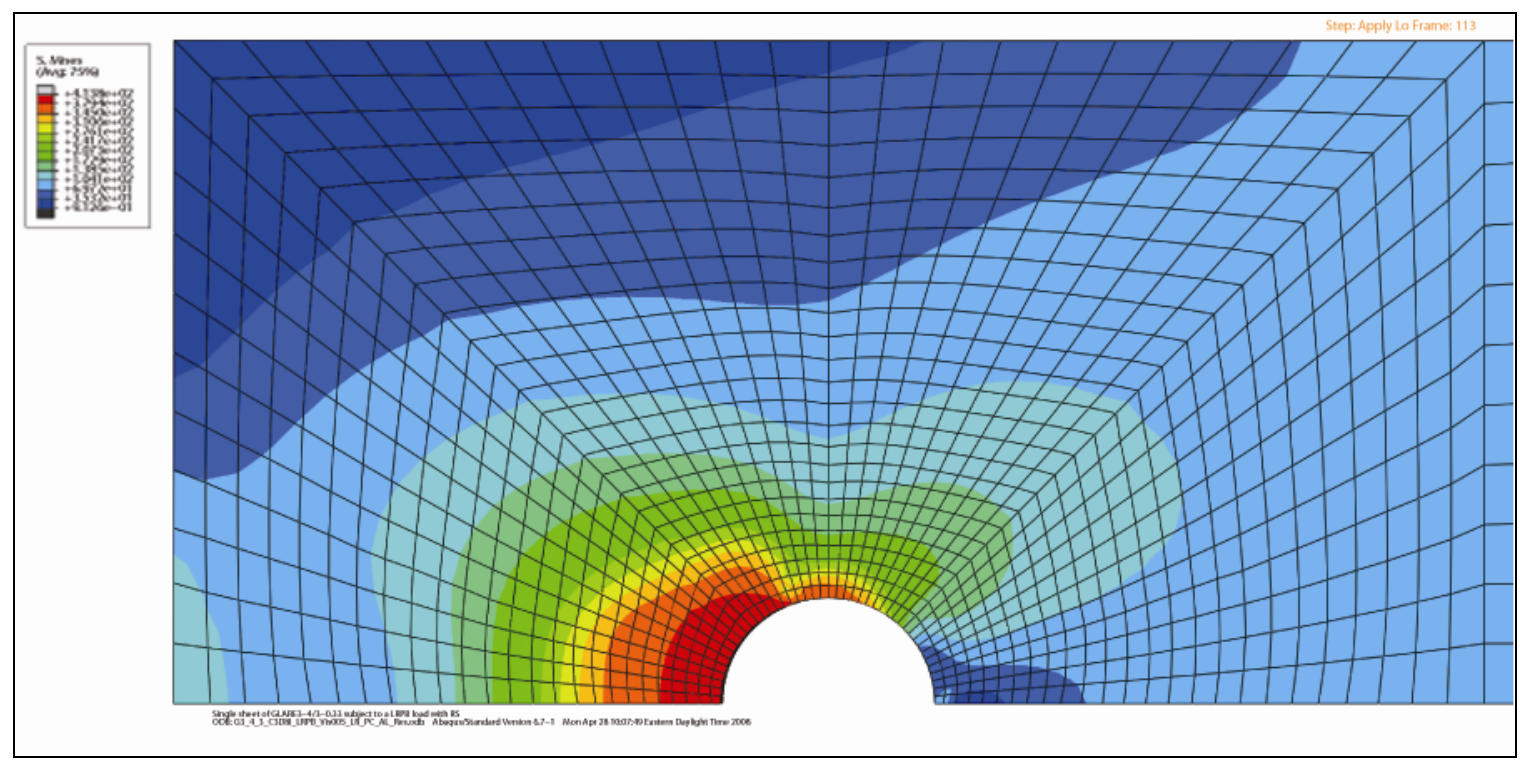

Figure 5.23: Distribution of the Von Mises stress field output variable in the aluminum layers resulting from the applied pin bearing load in a GLARE3-4/3-0.3 laminate.

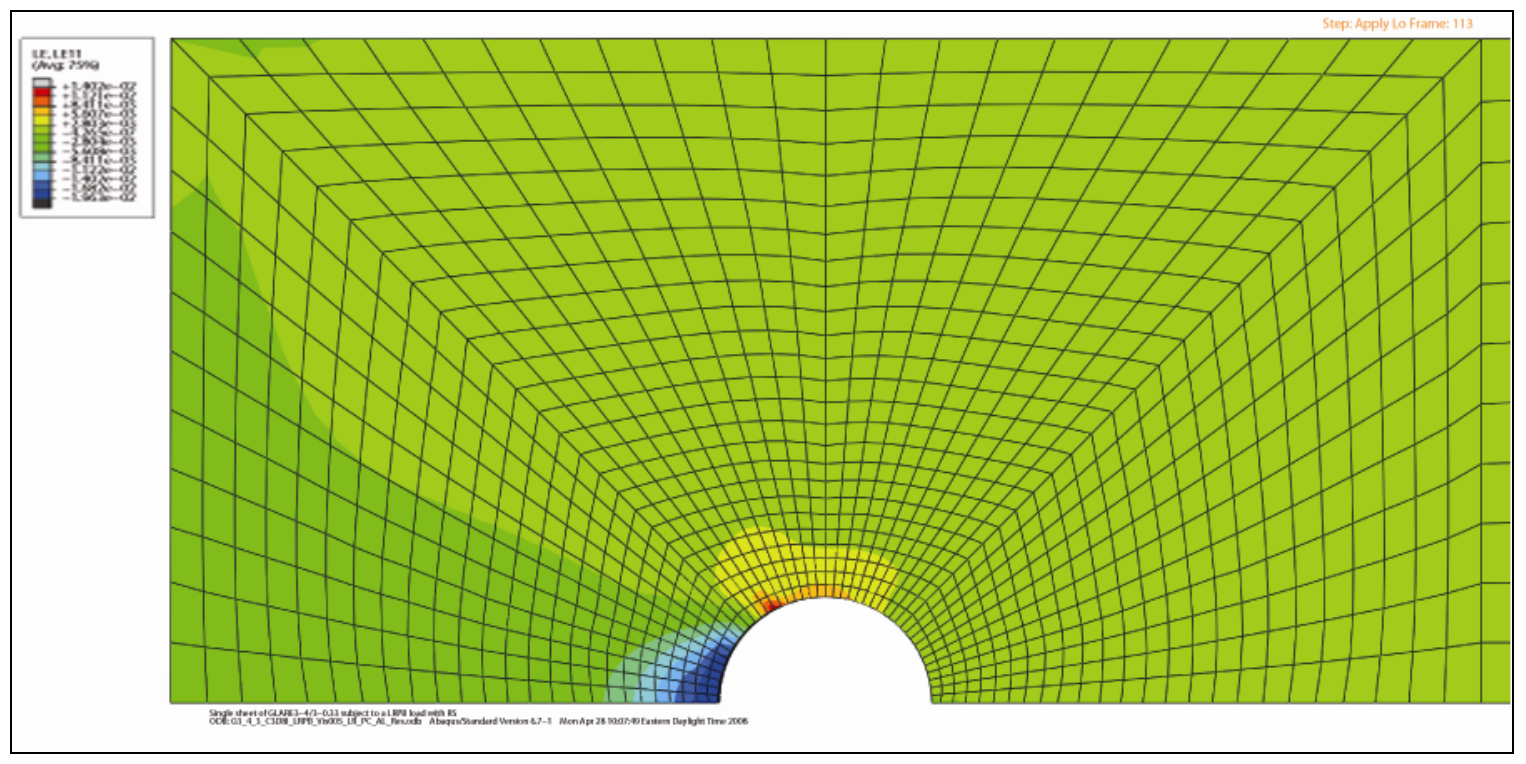

Figure 5.24: Distribution of the logarithmic strain in the longitudinal direction field output variable in the aluminum layers resulting from the applied pin bearing load in a GLARE3-4/3-0.3 laminate. 


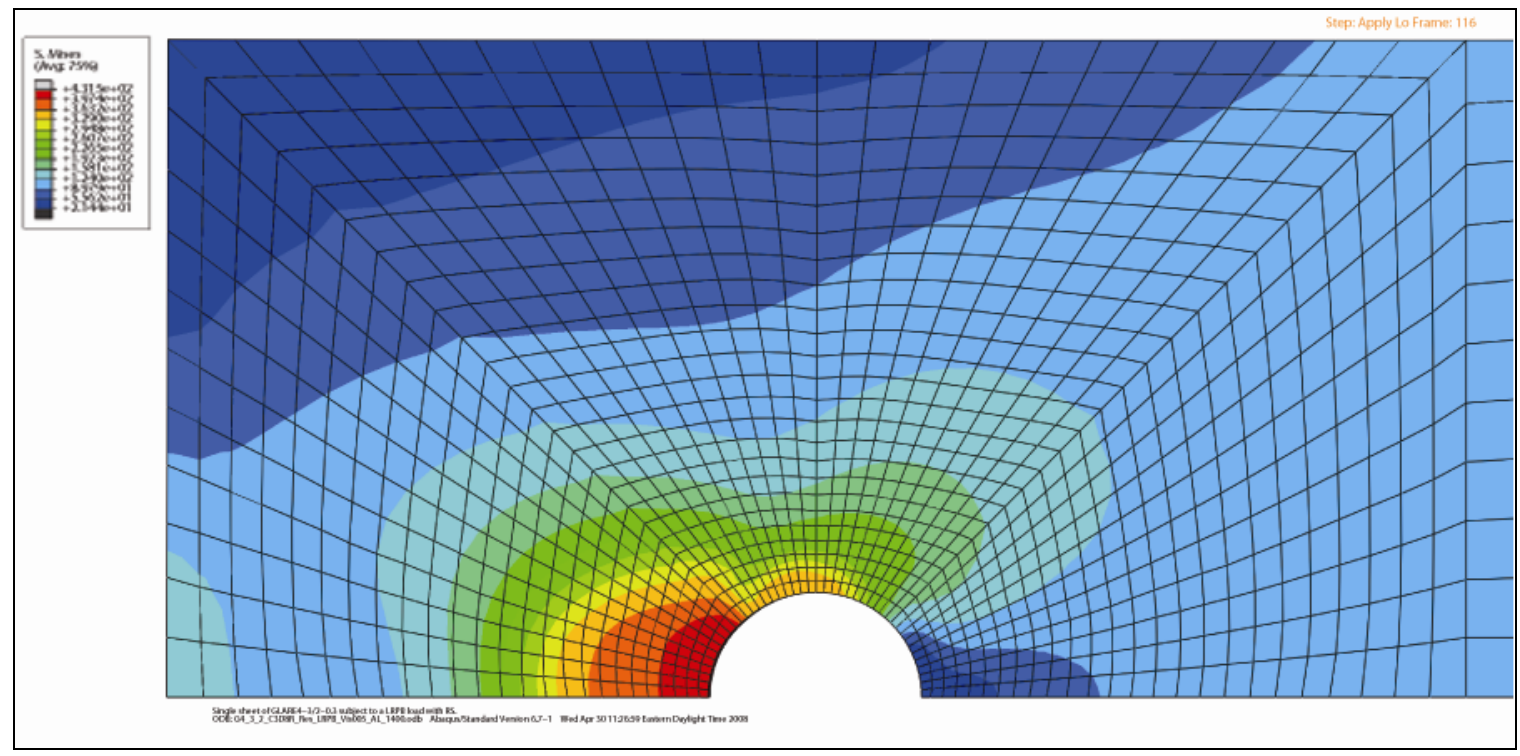

Figure 5.25: Distribution of the Von Mises stress field output variable in the aluminum layers resulting from the applied pin bearing load in a GLARE4-3/2-0.3 laminate.

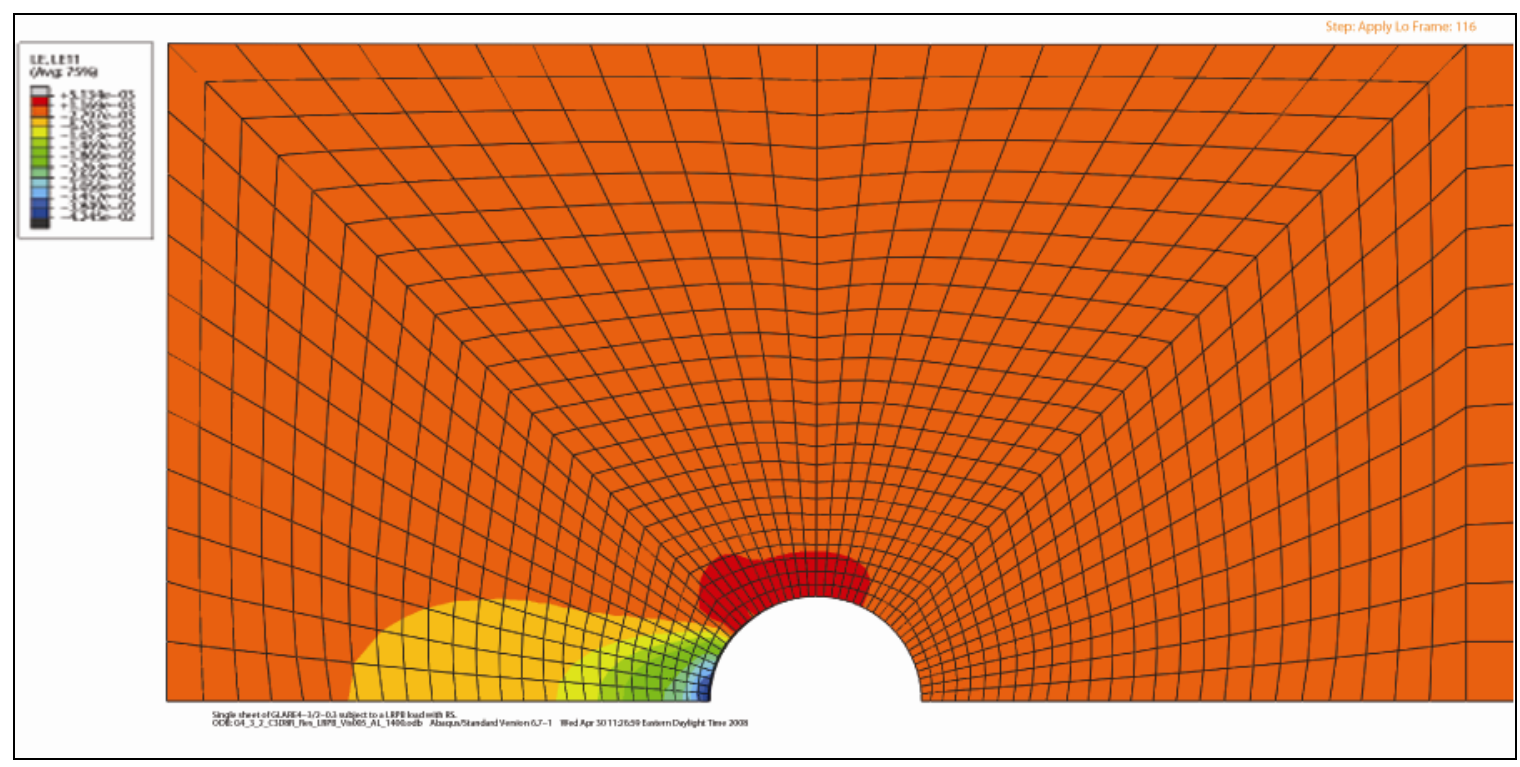

Figure 5.26: Distribution of the logarithmic strain in the longitudinal direction field output variable in the aluminum layers resulting from the applied pin bearing load in a GLARE4-3/2-0.3 laminate. 
noteworthy because it represents the strain experienced by the bonded strain gages in the experimental study.

In general, what is of interest in Figure 5.20 through Figure 5.26 is the highly localized stress/strain field directly adjacent to and moving away from the fastener hole. Recall that the YDB mechanism proposes that at the modified CCD - the distance in which the compressive stresses have fallen beneath the yield strength of the aluminum layers - all material within has yielded, delaminated and buckled, thus failing. When examining plots of the stress distribution it is vital to note the distance from the fastener hole in which the compressive stresses have indeed fallen beneath the yield strength of the aluminum and comparing that to the predicted modified CCD value of the previous analyses. Additional measures of determining yield and onset of plasticity at that same distance were also applied and will be discussed shortly. The important concept to note here is the occurrence of yielding and delamination at this threshold CCD distance. If the model predicts yielding and delamination that agrees well with the experimental measurement of yield at the same distance and also agrees with measurements of delamination from SEM imagery then the YDB mechanism would have empirical support. Yielding and buckling has already been experimentally investigated to occur at the modified CCD distance as discussed in Chapter 3 and therefore delamination is the only remaining portion of the YDB mechanism requiring experimental investigation. 


\subsubsection{Results from Additional Field Output Variables: ACYIELD, PE and PEEQ}

Aside from the typical field output variables used commonly in FE analyses, it was discussed previously that several very specific variables, each containing key information about the response of the laminate, were also requested. For the case of the aluminum layers examined now, the additional variables requested included the ACYIELD variable, the PE variable and the PEEQ variable.

The ACYIELD variable is an element status variable meaning it is active over the entire element incorporating influence from all of the integration points within it. What it measures is whether or not, according to the provided elastic-plastic material data, the aluminum is actively yielding at a specific time increment. For the case of the employed C3D8R element with its single integration point - a consequence of its reduced integration definition - it is a digital binary variable meaning that it either takes on a value of 1 or 0 making it very effective for mapping out regions where plasticity has occurred or not. For the other element employed, the incompatible mode C3D8I element, with its 8 integration points and additional modes including bending, values are grayscaled for each integration point from zero to even higher than one depending on the severity of the yielding. Consideration of this element allows for a useful measure of where the yielding approaches a nominal (approximately zero) value - in other words the modified CCD - in the model and can be compared to the previous predicted value. Since the case examined is that of a quasi-static load, the applied load step administers the load linearly over the total time range of the step. As a result, the use of a status variable can show the development of yield throughout the applied load window from onset to completion. For the analysis regarding ACYIELD conducted herein, outputs from both 
element types were considered for the sake of completion though little to no variation was observed.

The PE and PEEQ variables are two separate measurements of plasticity in the aluminum layers. PE represents all the plastic strain components of which the one of primary concern is the PE11 component - the component in the longitudinal direction - since as was the case for the logarithmic strain, the bonded strain gages were in line with this direction. The PEEQ variable is the equivalent plastic strain which is essentially a scalar measure of all the components of equivalent plastic strain at each position in the model. It can be loosely compared to the commonly employed Von Mises stress field output variable which is, simply put, a scalar measure of the equivalent stress at a point. Both of the plastic strain field output variables take on non-zero values only after the onset of plasticity, therefore in a location within the model where there is no plasticity (no yielding has occurred) both of these variables take on zero values. As a result they can be used as an effective mapping scheme between regions of induced plasticity and no plasticity; this is useful in the present case where the threshold of plasticity at the modified CCD is sought after.

Figure 5.27 through Figure 5.30 depict the distribution of the ACYIELD field output variable in the aluminum layers for all the variants analyzed. Note the high localization of yielded material which very closely resembles the pattern exhibited by the failed experimental specimens presented in Chapter 3.

It is visible by inspection once again that the yielded material has fallen within the modified CCD distance and is centralized near the notch and almost exclusively in the compressive stress region of interest as part of the YDB mechanism. However, it is 
possible to produce a much more precise measure of the actual yielded distance by employing the nodal path approach similar to the analysis performed in the CCD FE models previously. This approach, for obvious reasons, is much more desirable than one by inspection and was thus employed for enjoyment of its accuracy.

Figure 5.31 depicts a typical such nodal path plotted through an aluminum layer in-plane from which values of field output variables can be extracted with precision. These nodal paths were plotted through all the aluminum layers within the laminate and values for the field output variables were extracted and averaged to give a prediction which could be compared to the experimental results which showed that yielding had occurred at the modified CCD distance.

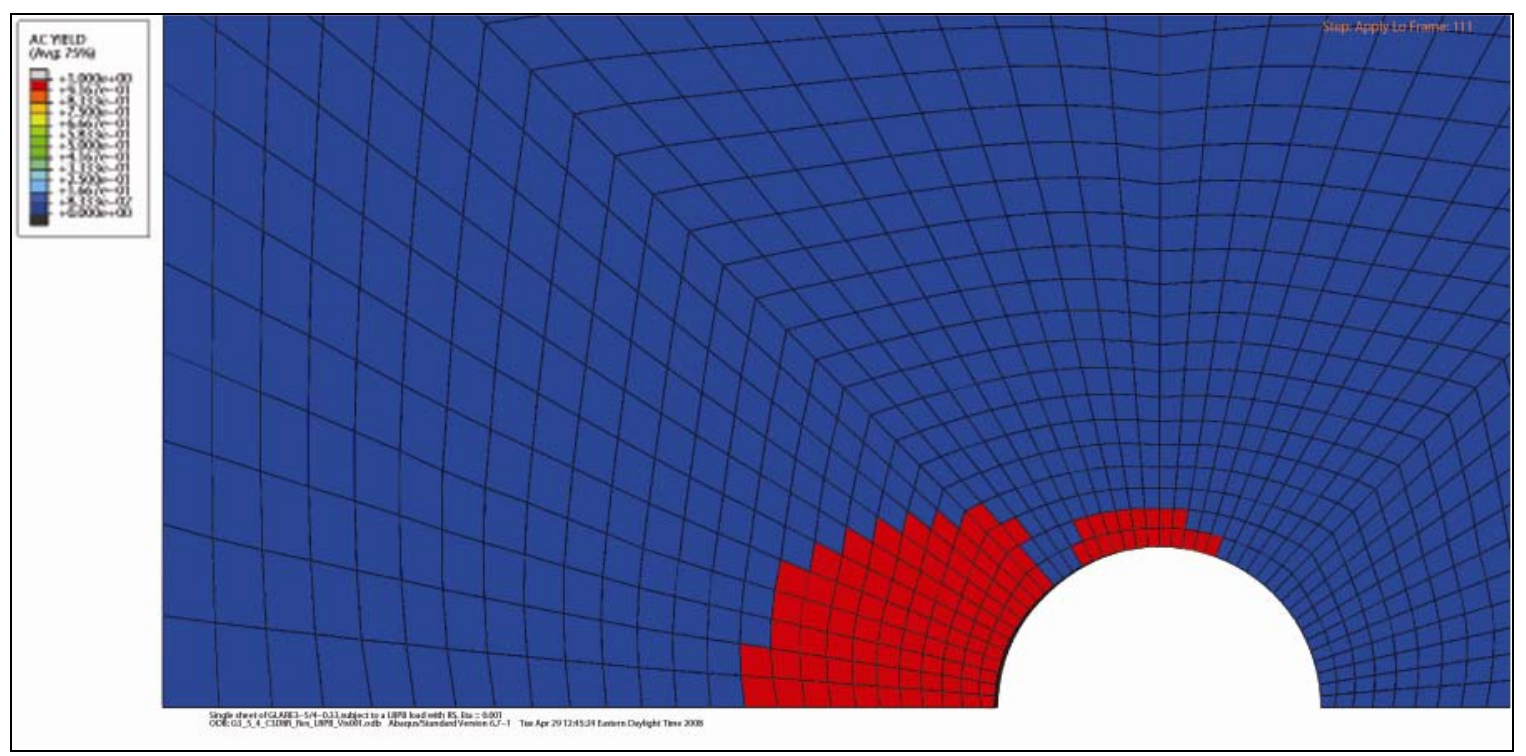

Figure 5.27: Distribution of the ACYIELD field output variable in the aluminum layers in a GLARE3-5/4-0.3 variant. Note the close similarity of the pattern of damage to the experimental specimens. 


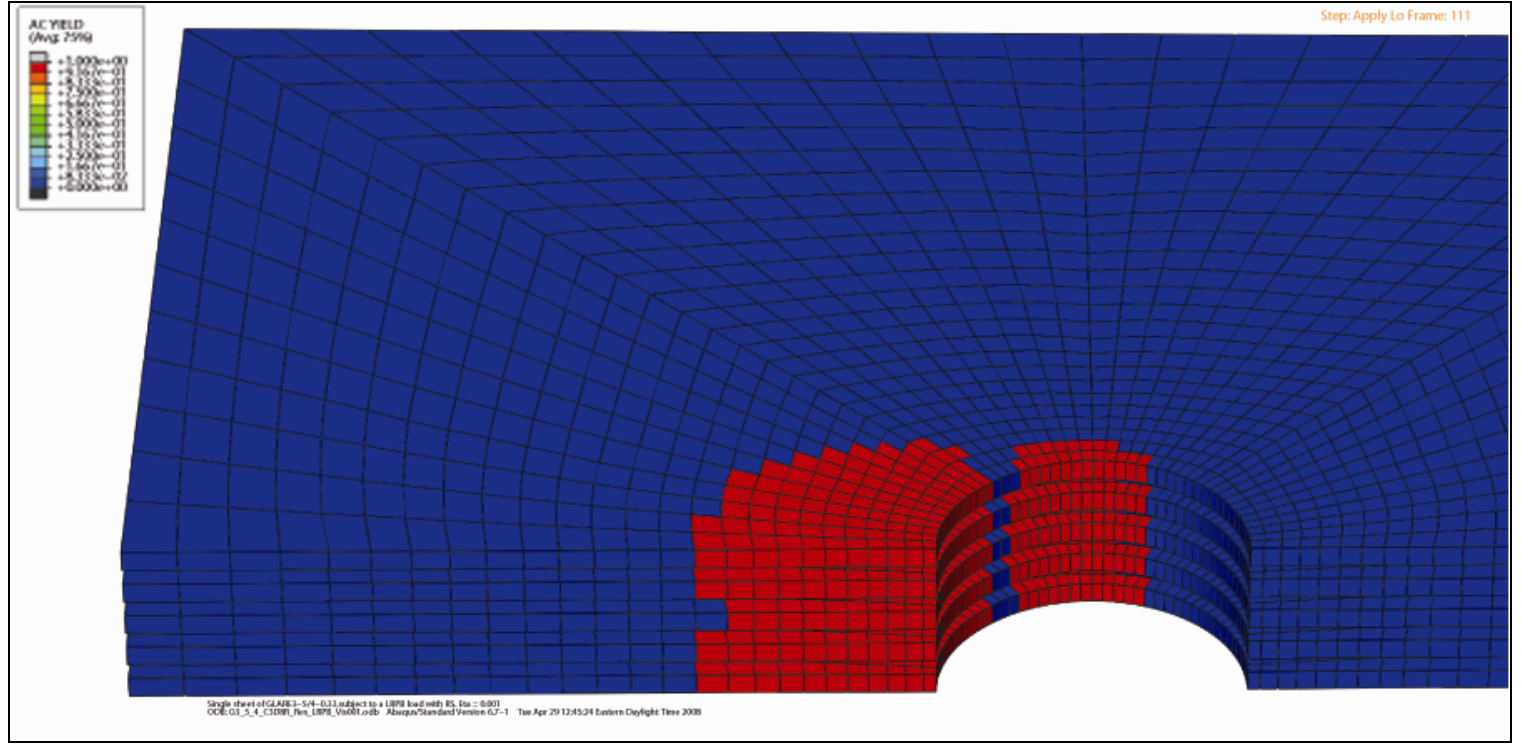

Figure 5.28: Through the thickness distribution of the ACYIELD field output variable in the aluminum layers of a GLARE3-5/4-0.3 variant.

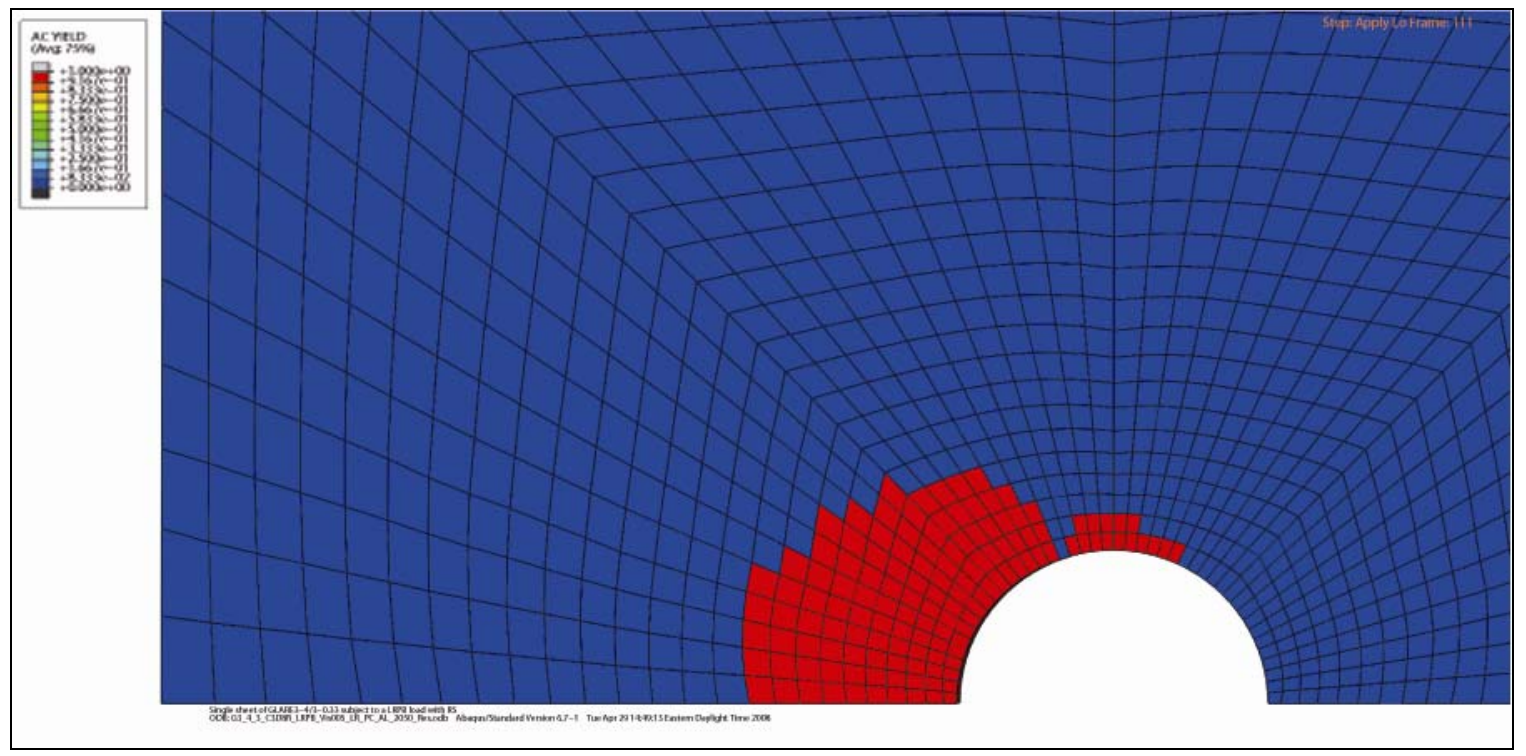

Figure 5.29: Distribution of the ACYIELD field output variable in the aluminum layers in a GLARE3-4/3-0.3 variant. 


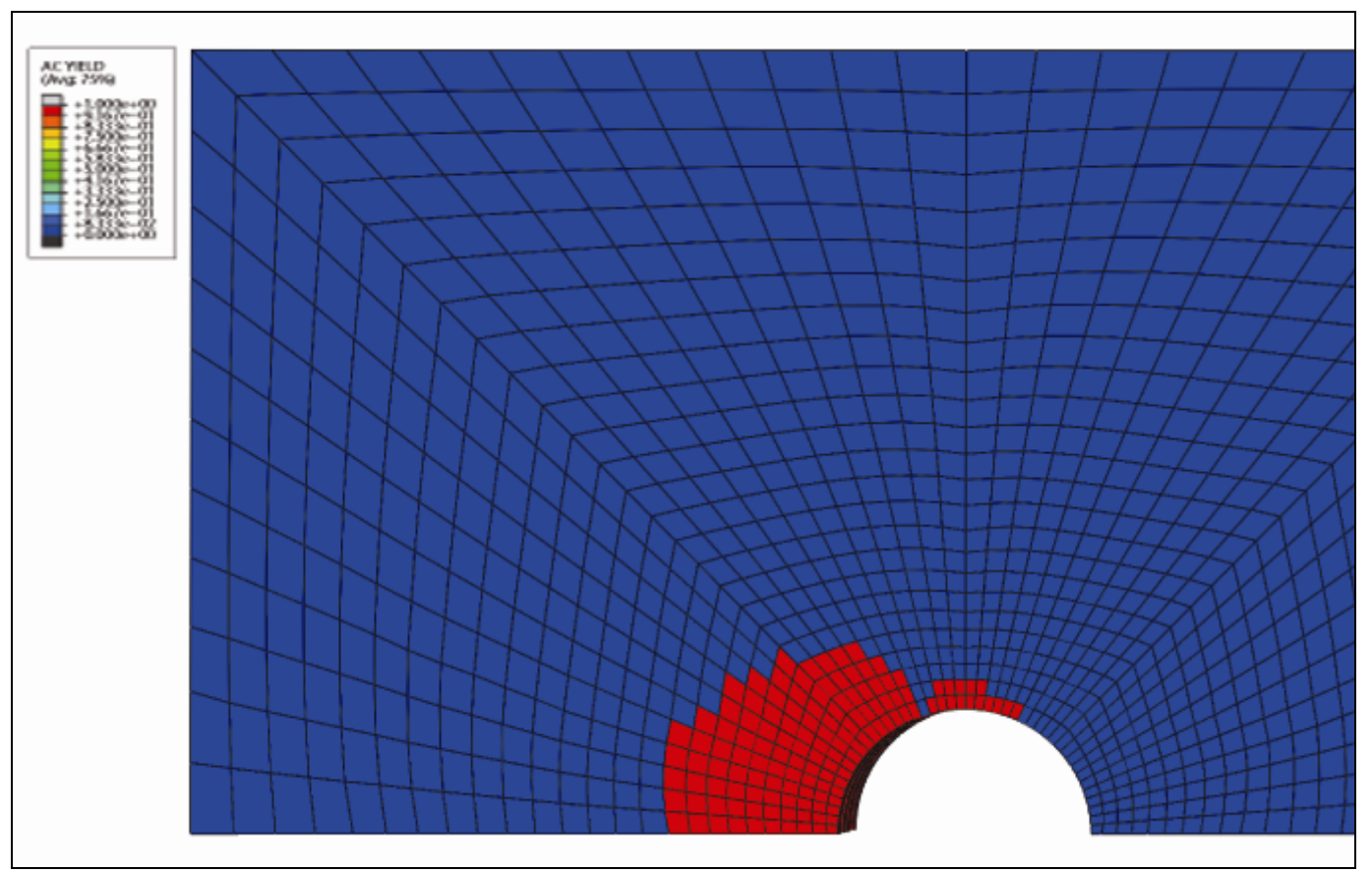

Figure 5.30: Distribution of the ACYIELD field output variable in the aluminum layers in a GLARE4-3/2-0.3 variant.

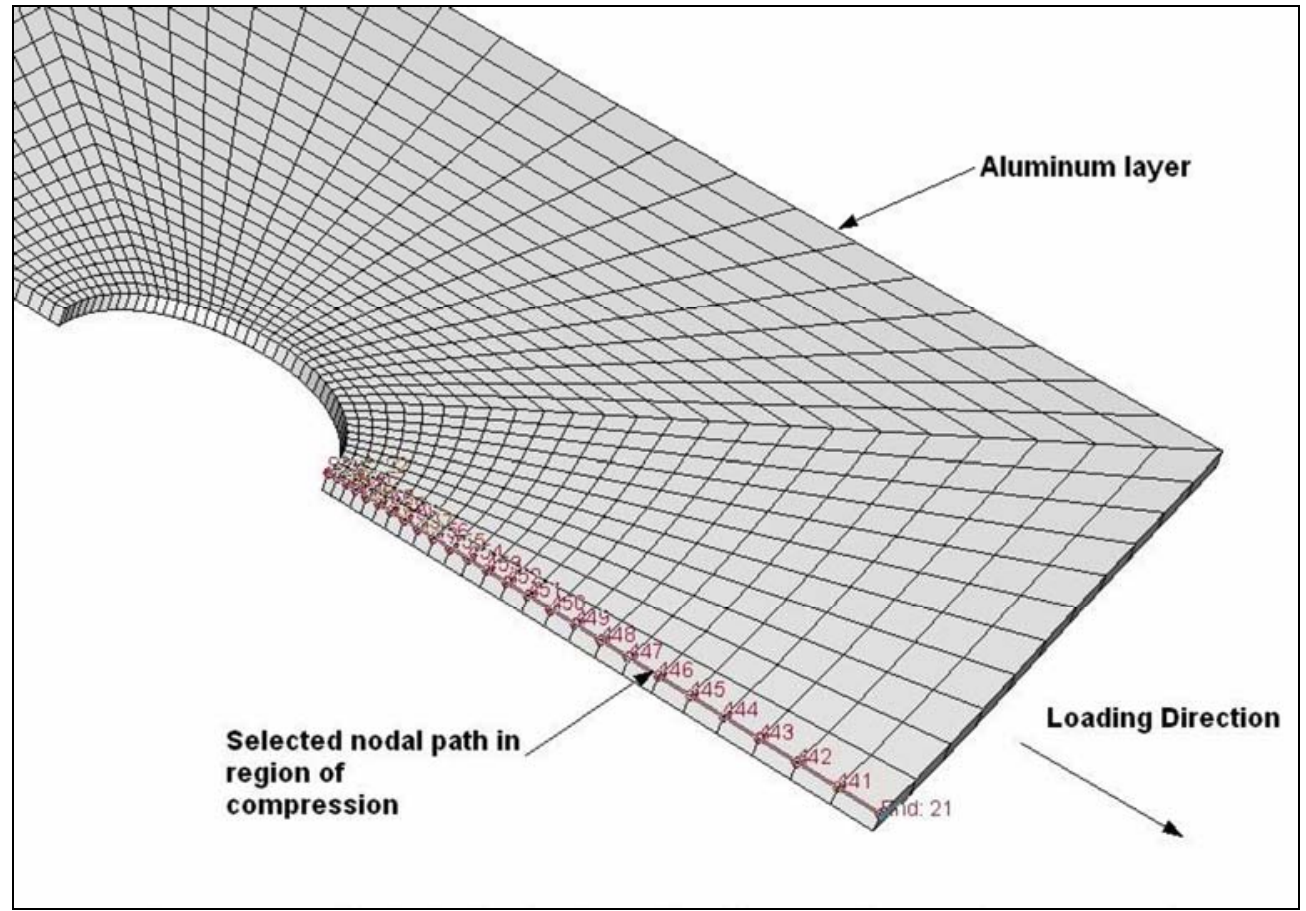

Figure 5.31: User defined nodal path within a typical aluminum layer used for the extraction of field output variable requests. 
In the case of the ACYIELD field output variable, values were taken from each integration point within the elements in one layer, extrema were then extracted from these values to give a proper measure of the spread of the variable within each element and then values from across the entire laminate were averaged to give the final value of yielded distance.

These values were then approximated through a minimization of least squares error and the nominal value for which yielding was determined to be inactive was set to $2.5 \%$ of the maximum value. From here the distance to which the ACYIELD variable has reached nominal far field values could be extracted with high precision. This analysis was performed via a custom written routine in Maple V10 [114] and an example of the routine can be found in the appendices.

Table 5.6 summarizes the distance to nominal ACYIELD values through the extraction of values at integration points, their extrema and subsequent averaging along the plotted nodal path for each of the aluminum layers within a GLARE4-3/2-0.3 laminate. Figure 5.32 through Figure 5.34 depict plots of the averaged ACYIELD values versus increasing distance from the fastener hole according to the routine just discussed for all of the variants analyzed. Finally, Table 5.7 tabulates the final calculated yield distance for the models and compares them to the predicted modified CCD values specific to each variant. 
Table 5.6: Summary of the extracted distances to nominal ACYIELD values and their relative error from the predicted modified CCD for a GLARE4-3/2-0.3 variant.

\begin{tabular}{ccc}
\hline Aluminum Layer & $\begin{array}{c}\text { Distance to Nominal } \\
\text { ACYIELD Value [mm] }\end{array}$ & $\begin{array}{c}\text { Relative Error from } \\
\text { Predicted Modified CCD [\%] }\end{array}$ \\
\hline Alum 1 - lower & 3.643 & -5.75 \\
\hline Alum 1 - upper & 3.997 & 3.40 \\
\hline Alum 2 - lower & 3.988 & 3.18 \\
\hline Alum 2 - upper & 3.988 & 3.18 \\
\hline Alum 3 - lower & 3.997 & 3.40 \\
\hline Alum 3 - upper & 3.643 & -5.75 \\
\hline Average of All Layers & 3.978 & 2.92 \\
\hline
\end{tabular}

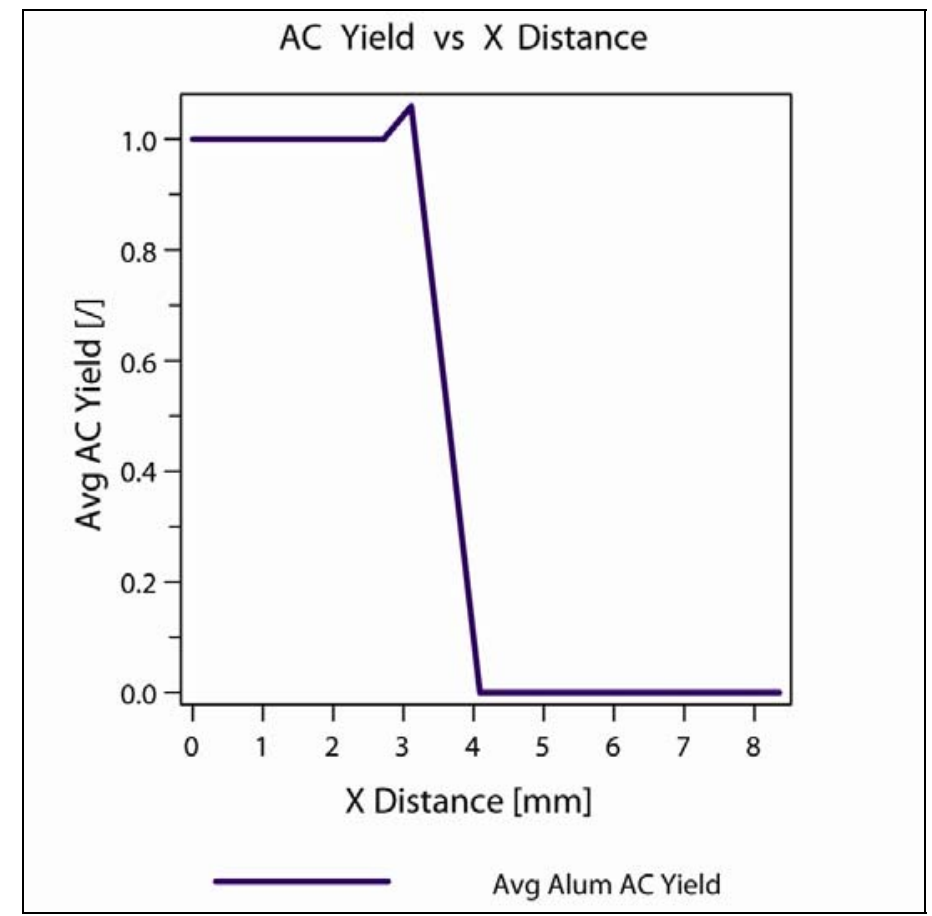

Figure 5.32: Plot of averaged ACYIELD versus distance from fastener hole for a GLARE3-5/4-0.3 variant. Note the distance to which the nominal value is reached. 


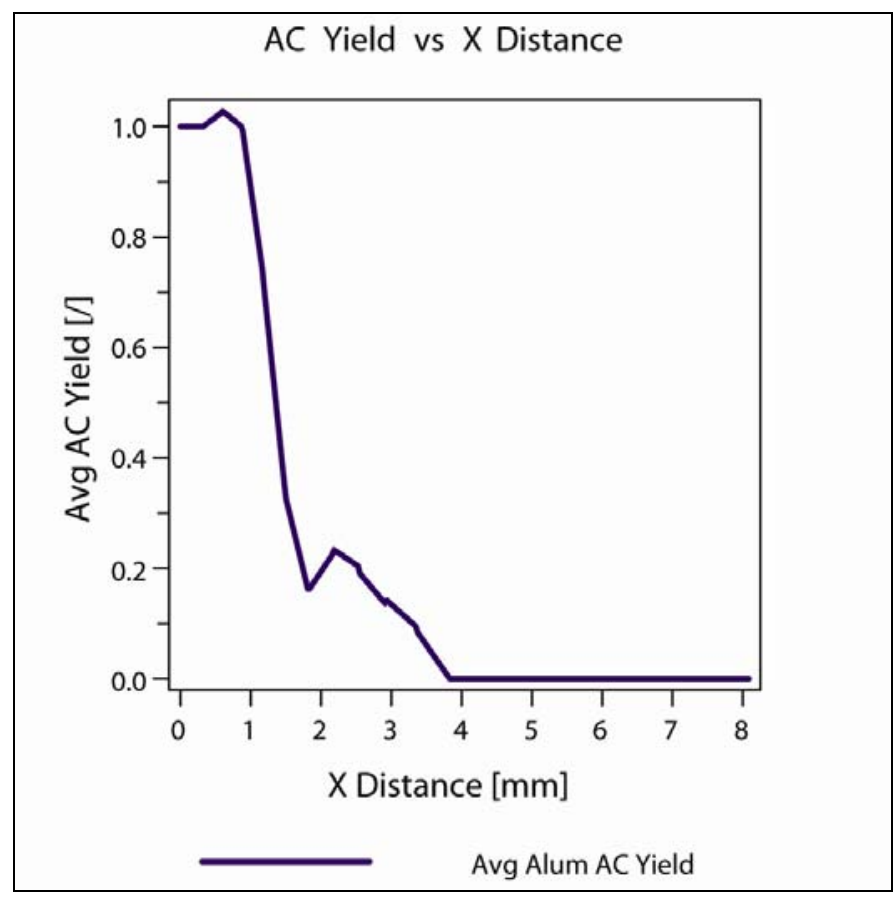

Figure 5.33: Plot of averaged ACYIELD versus distance from fastener hole for a GLARE3-4/3-0.3 variant. Note the distance to which the nominal value is reached.

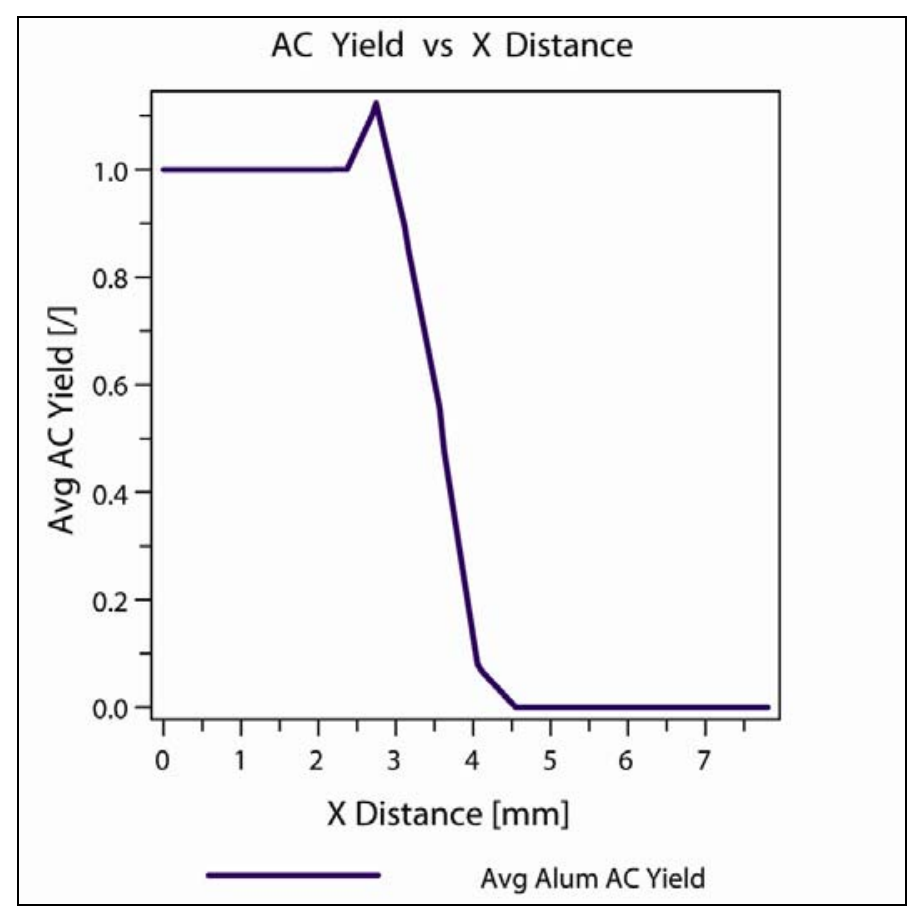

Figure 5.34: Plot of averaged ACYIELD versus distance from fastener hole for a GLARE4-3/2-0.3 variant. Note the distance to which the nominal value is reached. 
Table 5.7: Summary of the average distances to the nominal ACYIELD value and the relative error from the variant specific modified $C C D$ value for all variants.

\begin{tabular}{ccc}
\hline GLARE Variant & $\begin{array}{c}\text { Average Distance to } \\
\text { Nominal ACYIELD Value } \\
\text { [mm] }\end{array}$ & $\begin{array}{c}\text { Relative Error from } \\
\text { Predicted Modified CCD [\%] }\end{array}$ \\
\hline GLARE3-5/4-0.3 & 3.999 & 2.90 \\
\hline GLARE3-4/3-0.3 & 3.531 & 8.64 \\
\hline GLARE4-3/2-0.3 & 3.978 & 2.92 \\
\hline
\end{tabular}

Upon examination of Figure 5.32 through Figure 5.34 we see that the area of interest is the distance for which the value of the ACYIELD field output variable has reached a nominal state. This represents the threshold between yielding and non-yielding for the aluminum layers within the laminate. What occurs before this threshold, though interesting, is a value of ACYIELD above nominal and thus is not nearly of the same import as the threshold value.

An examination of the tabulated summary in Table 5.7 reveals a very small relative error between the modified CCD distance and the distance to nominal ACYIELD value. The error is as small as $2.90 \%$ and does not exceed $8.64 \%$ with an average value of $4.82 \%$. In reality, these distance calculations from the previous FE model and the present progressive damage FE model convey the same message. The end result is identical and they carry the same information only in the present case it was defined by a different field output variable.

Additional measures of plasticity is provided via the PE and PEEQ field output variables previously described and a nominal distance analysis was performed in an identical 
manner. Figure 5.35 through Figure 5.37 depict the distribution of both the PE and PEEQ field output variables within the aluminum layers for some of the variants analyzed.

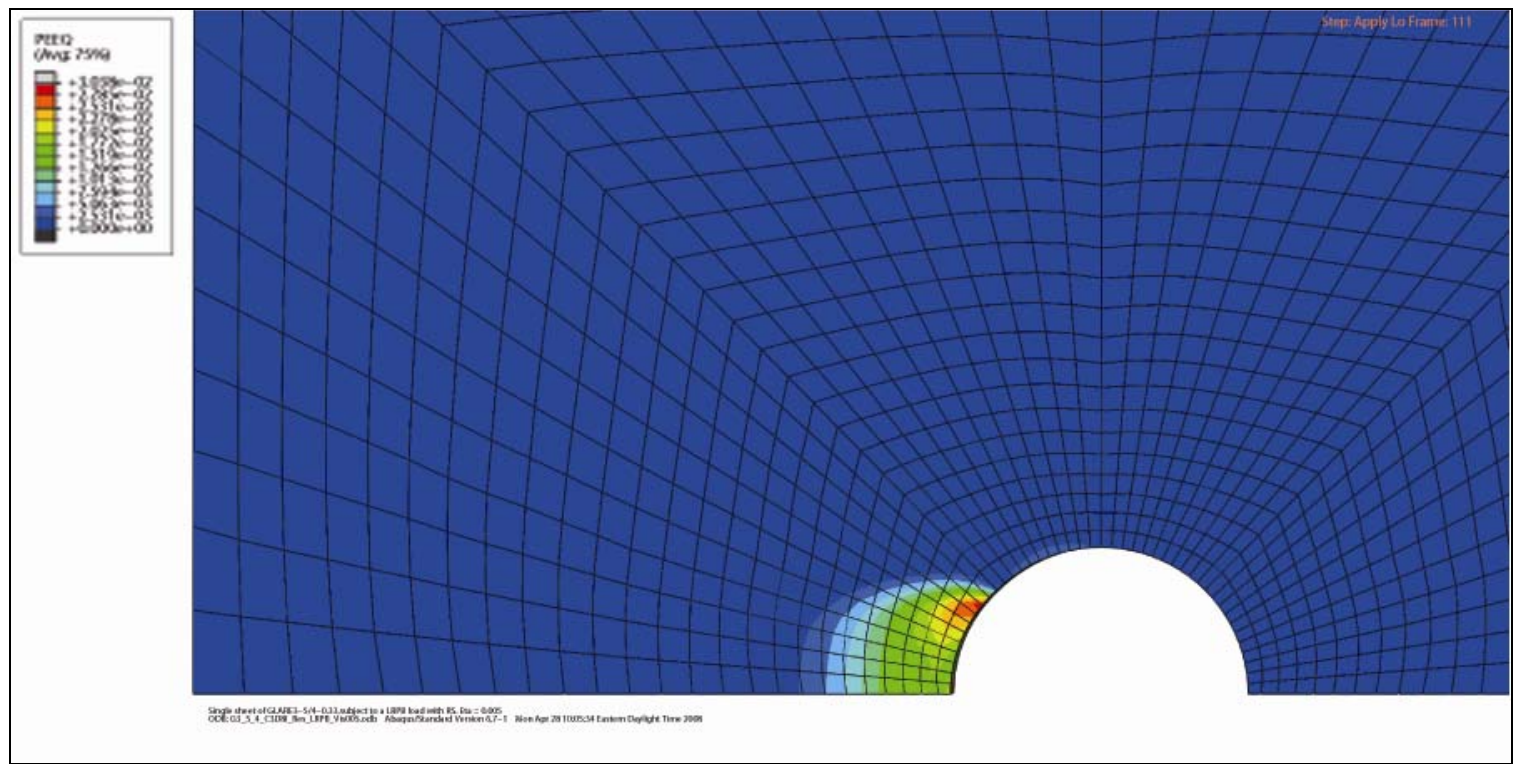

Figure 5.35: Distribution of the PEEQ field output variable within the aluminum layers of a GLARE3-5/4-0.3 variant.

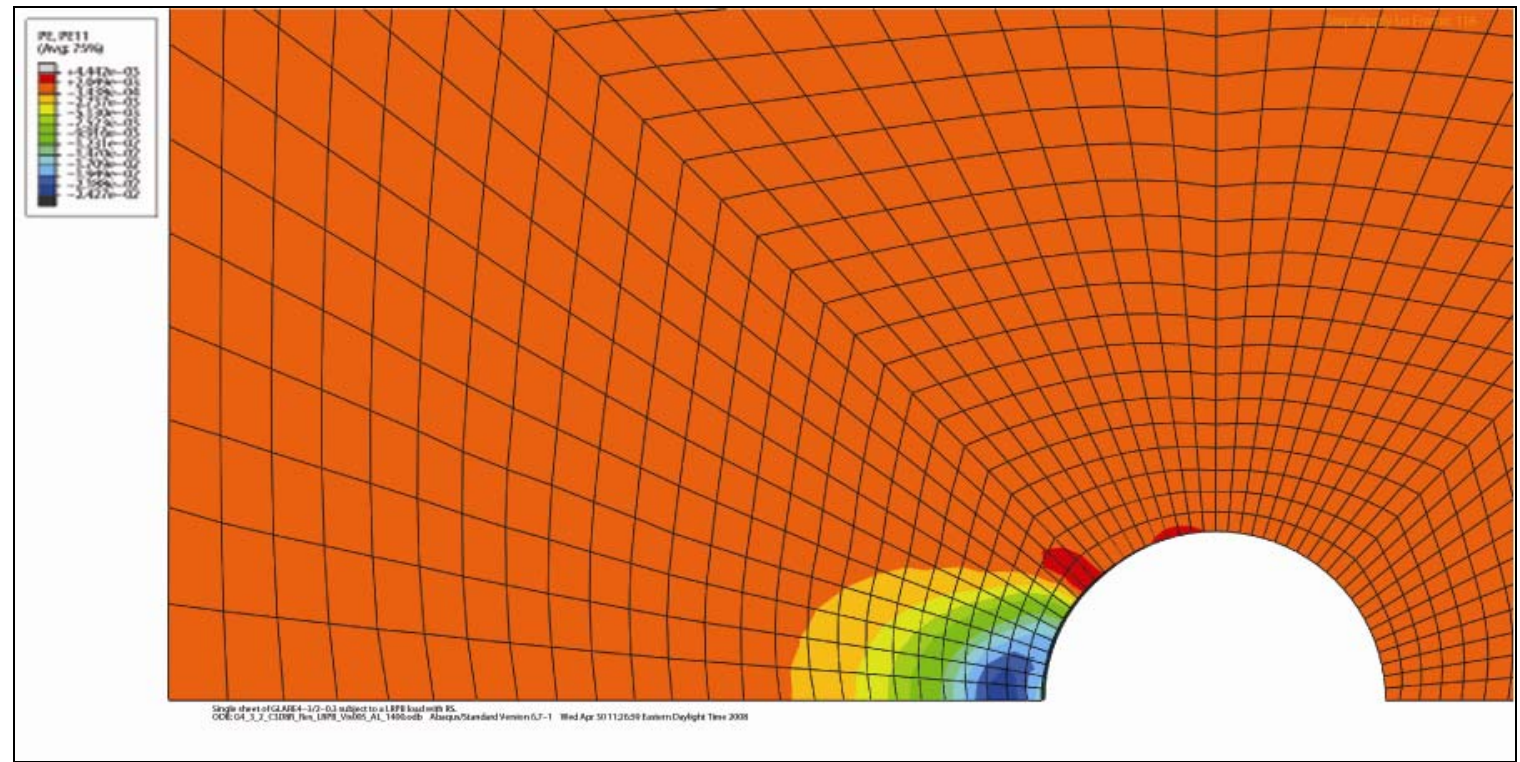

Figure 5.36: Distribution of the $P E$ field output variable within the aluminum layers of a GLARE4-3/2-0.3 variant. 


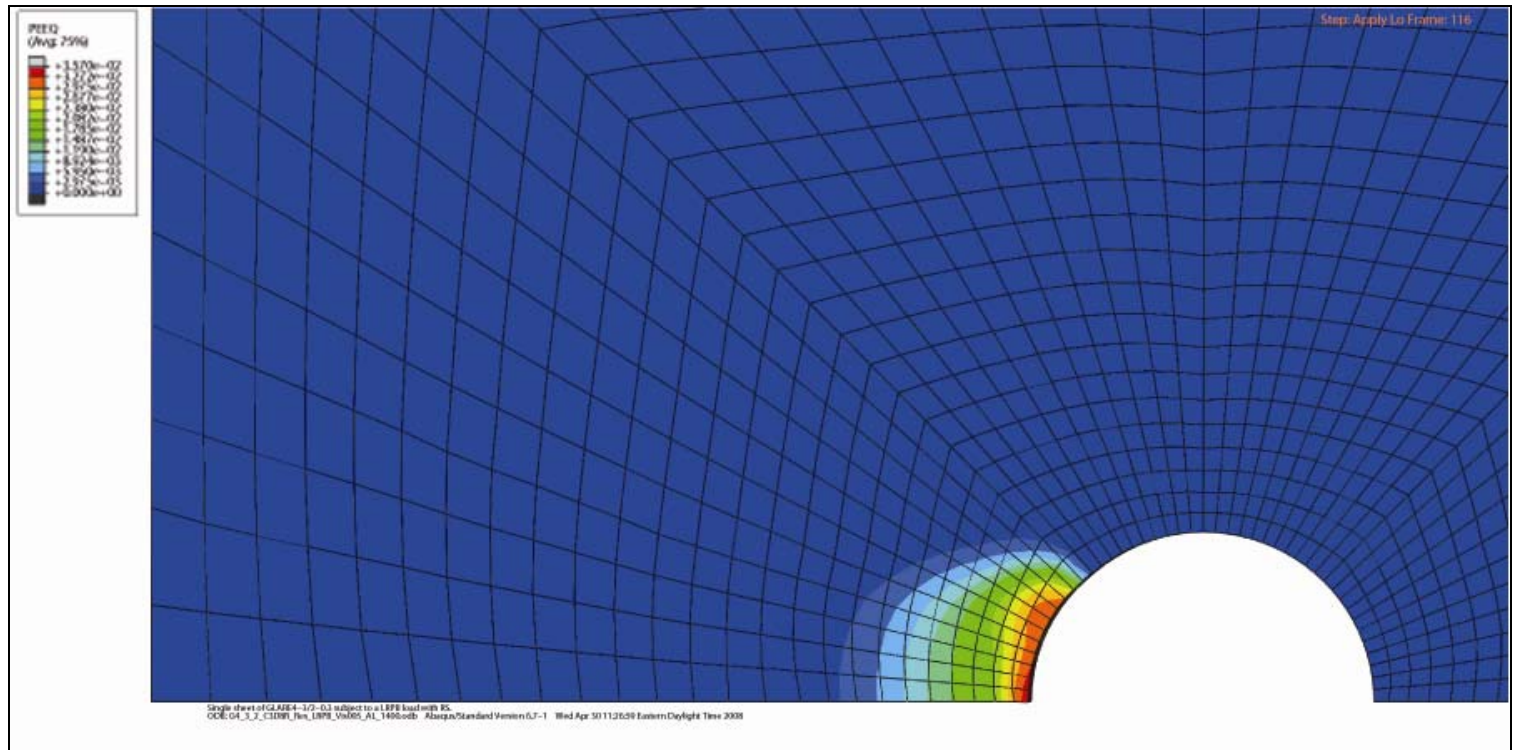

Figure 5.37: Distribution of the PEEQ field output variable within the aluminum layers of a GLARE4-3/2-0.3 variant.

Table 5.8 shows the layer by layer extraction of values at integration points and the subsequent averaging for the distance to nominal value of a GLARE4-3/2-0.3 variant. In the case of the PE and PEEQ field output variables, the nominal value for which plasticity was deemed no longer was set to a value of 100 microstrain. This is a very small value which from an empirical point of view where the strains experienced by the strain gages approached 20,000 microstrain, we see that it represents a mere $0.5 \%$ and thus can be deemed nominal indeed.

Figure 5.38 through Figure 5.40 are plots of both averaged PE and PEEQ values versus increasing distance from the fastener hole. Once again, what is of importance is the distance until nominal values are reached which is similar to the modified CCD but now from a strain approach rather than a stress approach. 
Table 5.8: Summary of the extracted distances to nominal PE and PEEQ values and their relative error from the predicted modified CCD for a GLARE4-3/2-0.3 variant.

\begin{tabular}{ccccc}
\hline Aluminum Layer & $\begin{array}{c}\text { Distance to } \\
\text { Nominal } \\
\text { PE11 Value } \\
\text { [mm] }\end{array}$ & $\begin{array}{c}\text { Relative Error } \\
\text { from } \\
\text { Predicted } \\
\text { Modified CCD } \\
\text { [\%] }\end{array}$ & $\begin{array}{c}\text { Distance to } \\
\text { Nominal } \\
\text { PEEQ Value } \\
\text { [mm] }\end{array}$ & $\begin{array}{c}\text { Relative Error } \\
\text { from Predicted } \\
\text { Modified CCD [\%] }\end{array}$ \\
\hline Alum 1 & 4.023 & 4.08 & 4.027 & 4.16 \\
\hline Alum 2 & 3.586 & -7.23 & 3.586 & -7.23 \\
\hline Alum 3 & 4.023 & 4.08 & 4.027 & 4.16 \\
\hline Average of All Layers & 3.999 & 3.44 & 4.003 & 3.57 \\
\hline
\end{tabular}

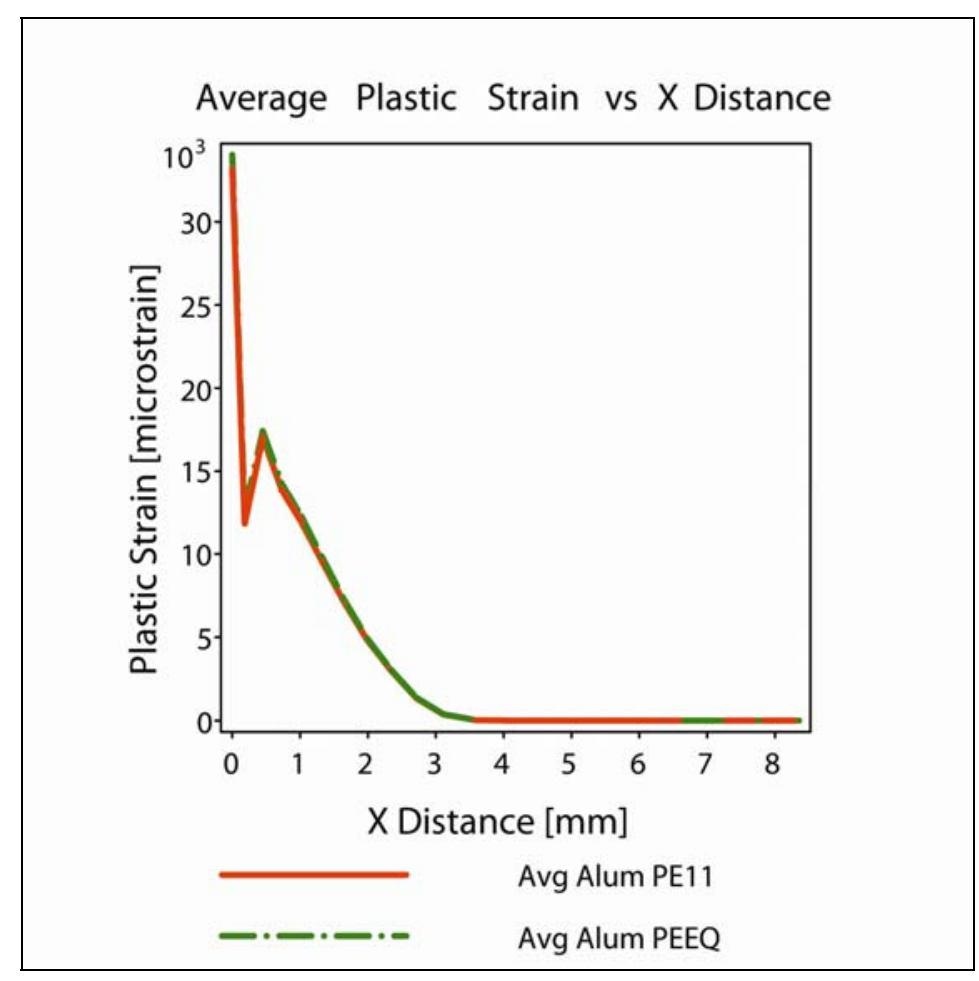

Figure 5.38: Plot of averaged PE and PEEQ versus distance from fastener hole for a GLARE3-5/4-0.3 variant. 


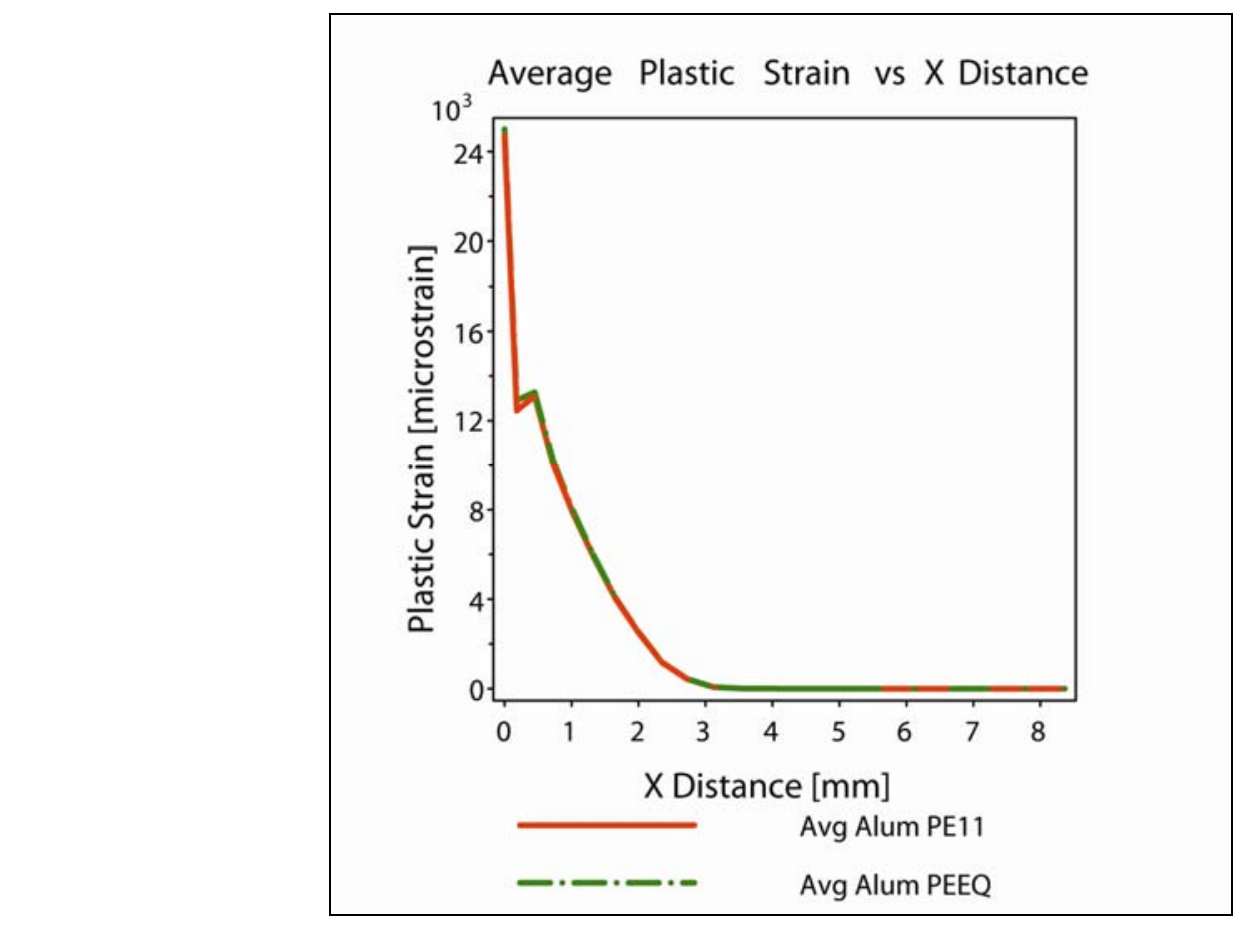

Figure 5.39: Plot of averaged $P E$ and PEEQ versus distance from fastener hole for a GLARE3-4/3-0.3 variant.

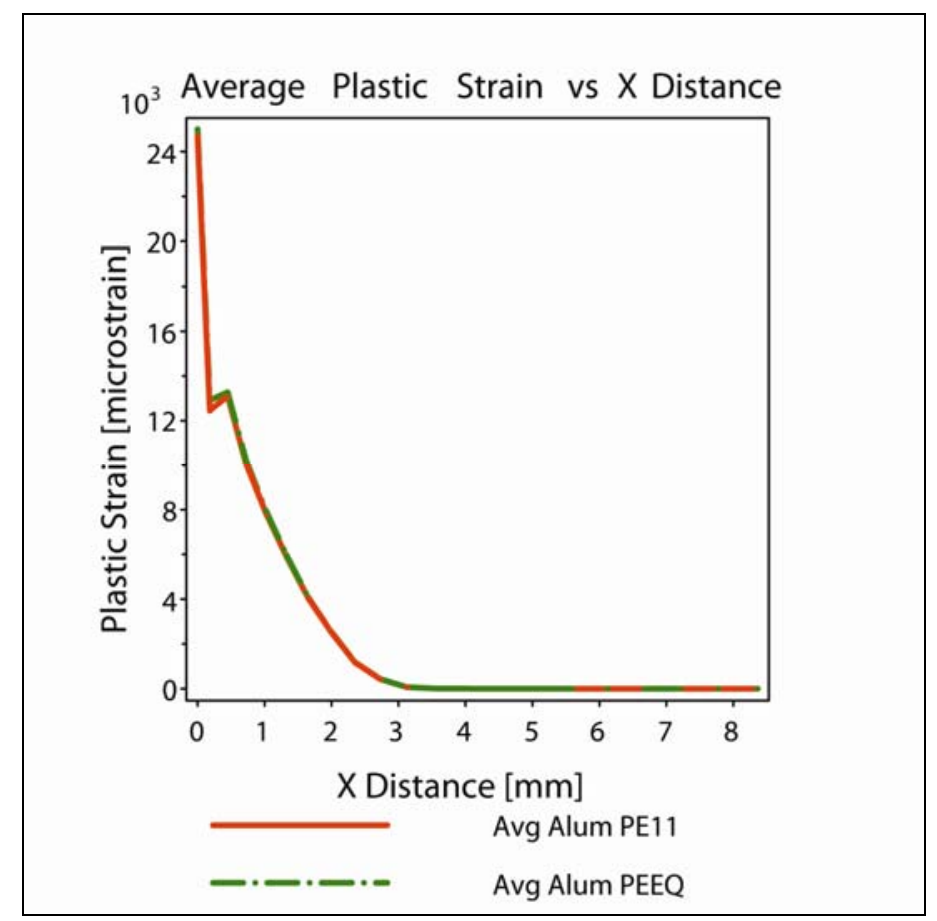

Figure 5.40: Plot of averaged PE and PEEQ versus distance from fastener hole for a GLARE4-3/2-0.3 variant. 
Table 5.9 shows the tabulated the final calculated yield distance according to PE and PEEQ field output variables for the models and compares them to the predicted modified CCD values specific to each variant. Here we see yet again a very small relative error from the predicted modified CCD value as previously defined but now arrived at via a completely difference approach and set of field output variables.

Table 5.9: Summary of the average distances to the nominal $P E$ and PEEQ value and the relative error from the variant specific modified $C C D$ value for all variants.

\begin{tabular}{ccccc}
\hline GLARE Variant & $\begin{array}{c}\text { Distance to } \\
\text { Nominal } \\
\text { PE11 Value } \\
\text { [mm] }\end{array}$ & $\begin{array}{c}\text { Relative Error } \\
\text { from } \\
\text { Predicted } \\
\text { Modified CCD } \\
\text { [\%] }\end{array}$ & $\begin{array}{c}\text { Distance to } \\
\text { Nominal } \\
\text { PEEQ Value } \\
\text { [mm] }\end{array}$ & $\begin{array}{c}\text { Relative Error } \\
\text { from Predicted } \\
\text { Modified CCD [\%] }\end{array}$ \\
\hline GLARE3-5/4-0.3 & 3.554 & -8.52 & 3.558 & -8.41 \\
\hline GLARE3-4/3-0.3 & 3.568 & -7.69 & 3.569 & -7.66 \\
\hline GLARE4-3/2-0.3 & 3.999 & 3.44 & 4.003 & 3.56 \\
\hline
\end{tabular}

\subsubsection{Results and Discussion for the Glass Layers}

Figure 5.41 and Figure 5.42 depict the Von Mises stress distribution within the glass layers as a result of the applied load for the GLARE3-4/3-0.3 variant. What is noteworthy here is the localization of stress again near the notch. In addition, the peak stresses in the glass layers correspond to their principal orthotropic direction with the longitudinal and transverse layers each showing additional peak zones due to the in-plane shear induced by the pin bearing load, though in different locations reflecting their bias in stiffness. 


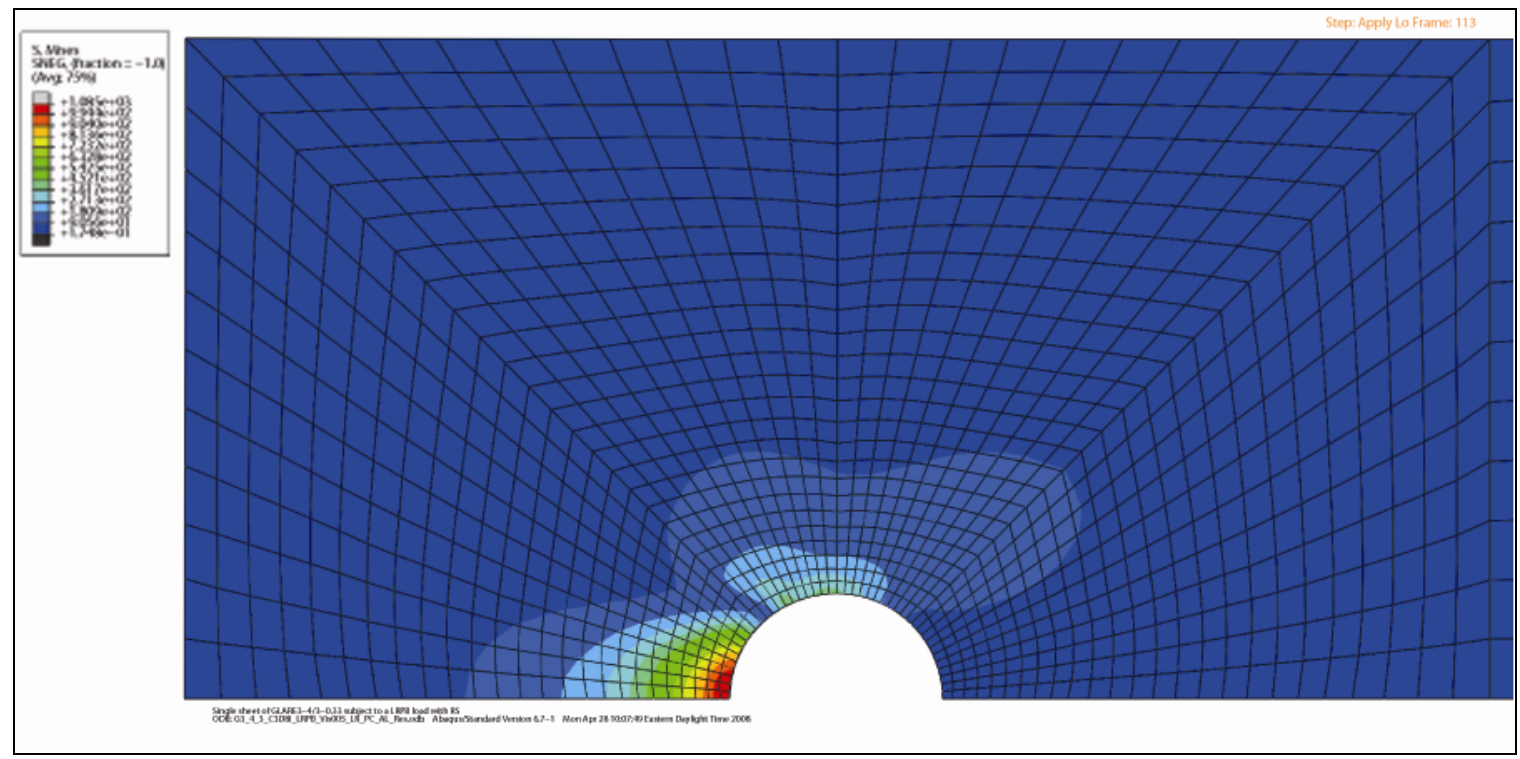

Figure 5.41: Distribution of the Von Mises stress field output variable for a longitudinal glass prepreg layer in a GLARE3-4/3-0.3 variant.

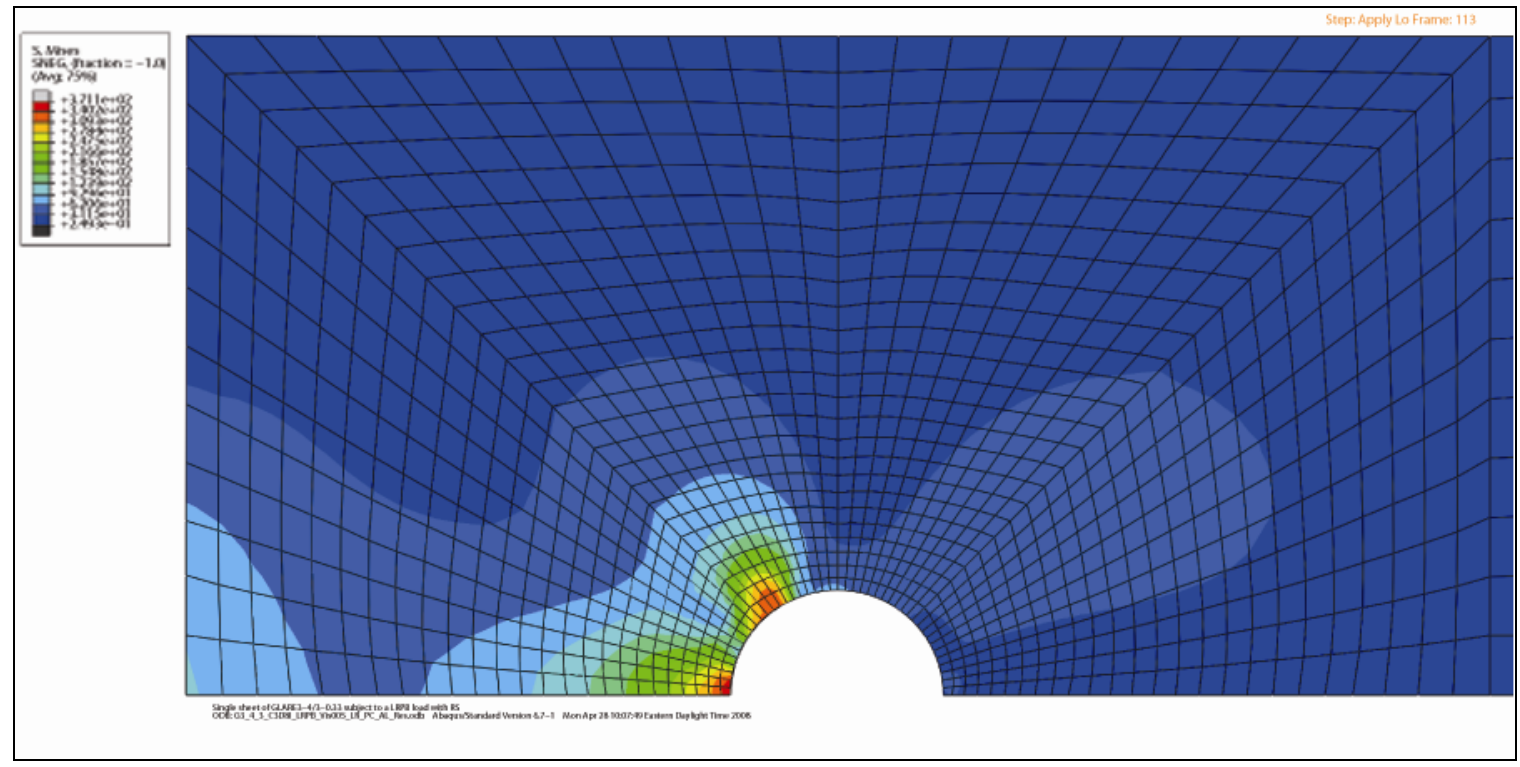

Figure 5.42: Distribution of the Von Mises stress field output variable for a transverse glass prepreg layer in a GLARE3-4/3-0.3 variant. 
Of more interest though is the distribution of the four damage variables requested for in the analysis; recall they are: fibre tension (HSNFTCRT), fibre compression (HSNFCCRT), matrix tension (HSNMRCRT) and matrix compression (HSNMTCRT).

Figure 5.43 through Figure 5.45 are selected depictions of these distributions as analyzed. Of these figures, the most noteworthy ones are the depictions of the fibre compression damage variable and the matrix damage variable in both compression and tension. In all cases we see that the compressive loading results in failure in all of the glass layers with slightly different distributions depending upon fibre orientation. In the case of both the matrix damage variables, we see complete failure for a large region adjacent to the laminate notch. This supports the notion that the glass layers will buckle due to a lack of lateral support which would have been provided by the aluminum layers (now delaminated as will be shown next) and the now failed matrix which would have provided additional compressive strength which the fibres lack on their own.

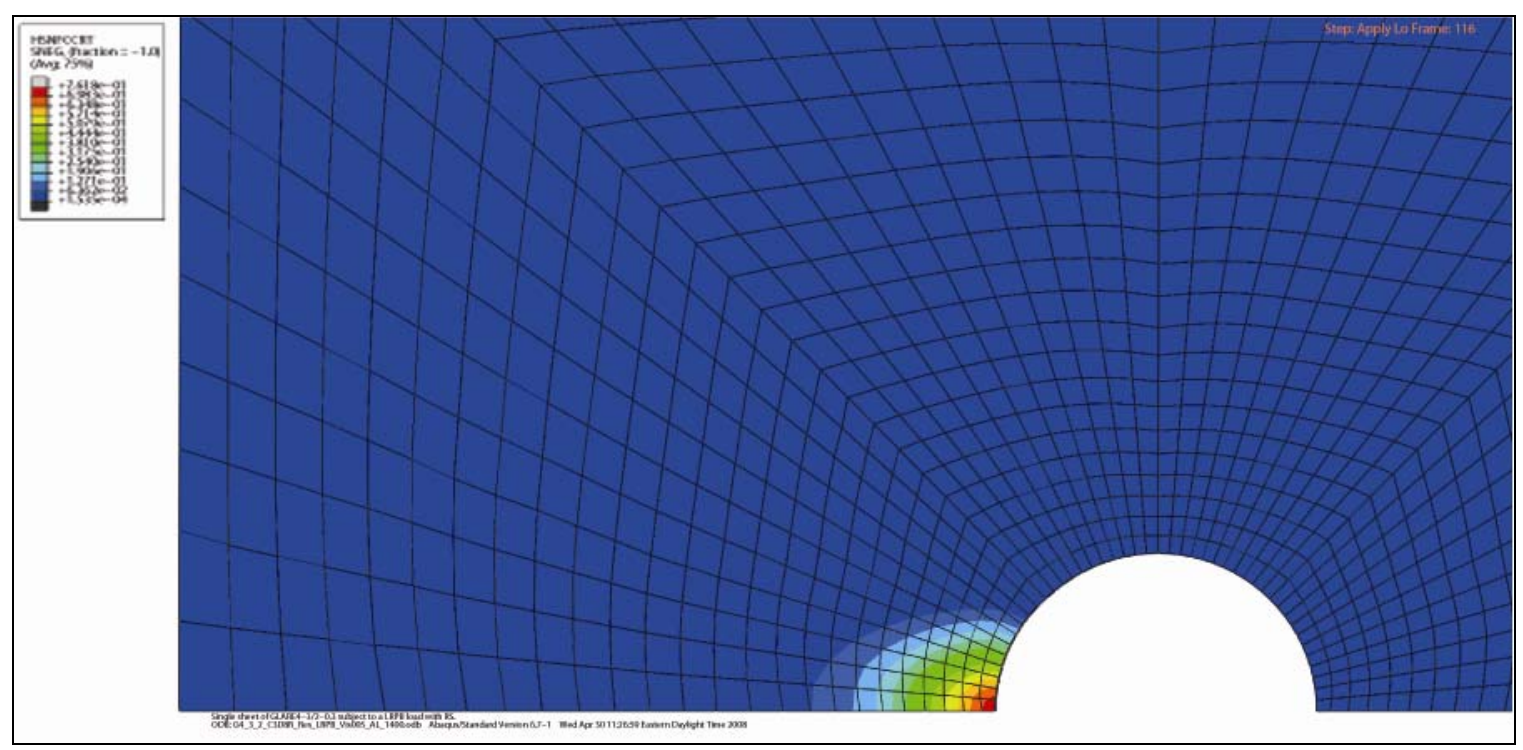

Figure 5.43: Distribution of the HSNFCCRT field output variable in a longitudinal glass prepreg layer of a GLARE4-3/2-0.3 variant. 


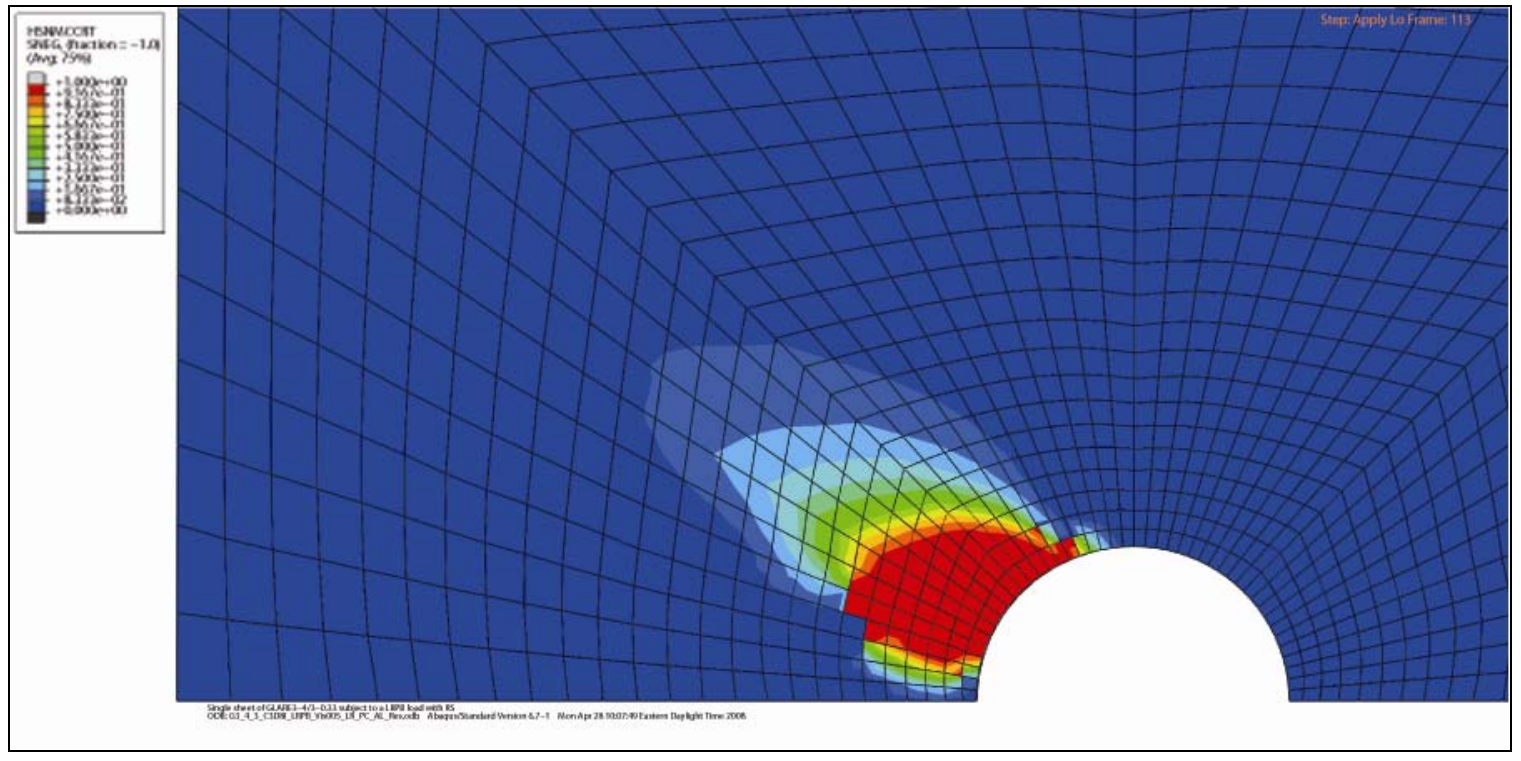

Figure 5.44: Distribution of the HSNMCCRT field output variable in a longitudinal glass prepreg layer of a GLARE3-4/3-0.3 variant.

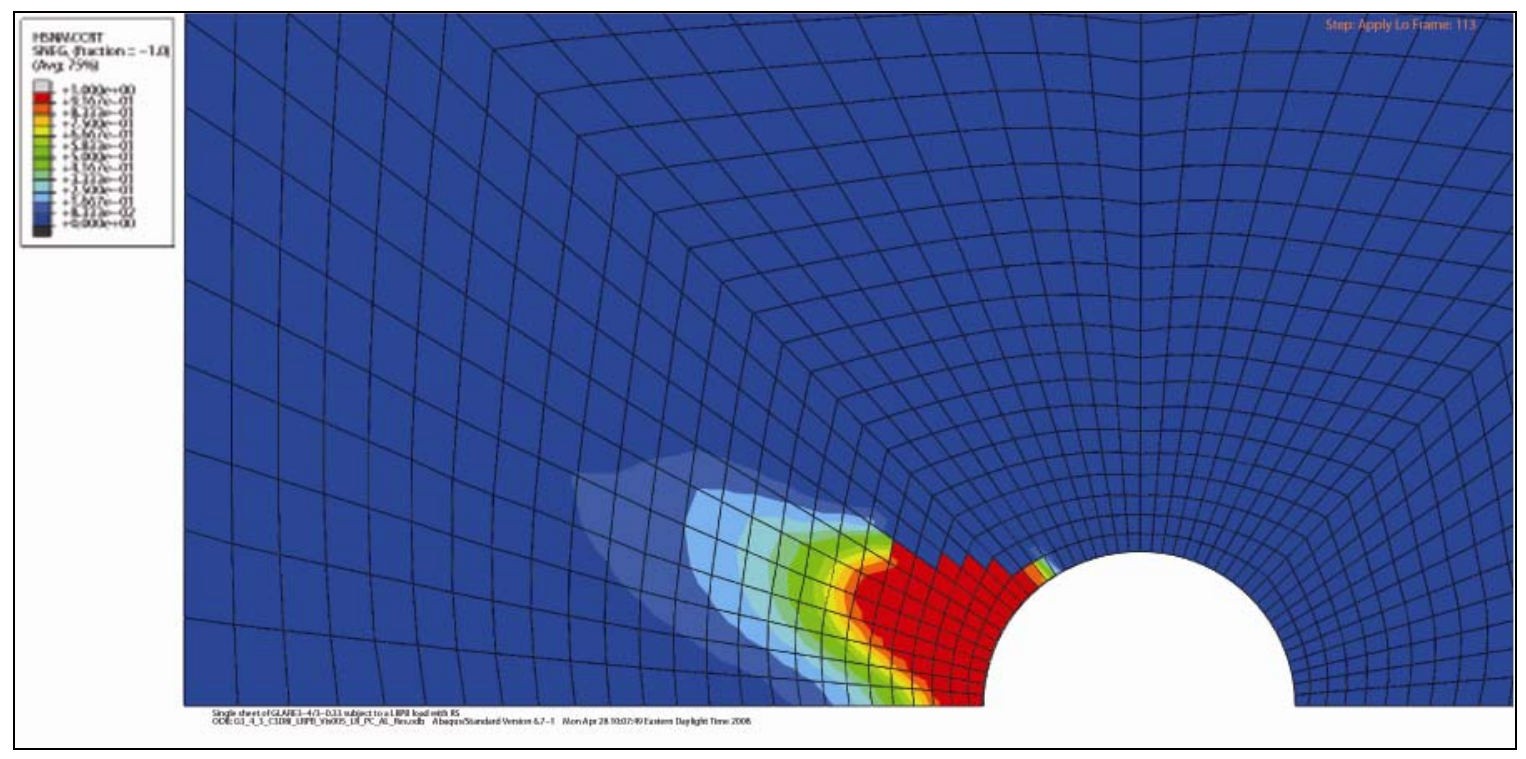

Figure 5.45: Distribution of the HSNMCCRT field output variable in a transverse glass prepreg layer of a GLARE3-4/3-0.3 variant. 


\subsubsection{Results and Discussion of the Delamination Modeling}

As mentioned at the end of section 5.2.2, both yielding and buckling as part of the YDB mechanism have already been experimentally investigated. Therefore, only delamination remains to be investigated. To accomplish this, an examination of the delaminated area as measured by the QUADECRT field output variable was conducted, as was a comparison to SEM imagery of failed pin bearing specimens.

Figure 5.46 depicts the typical distribution of the Von Mises stress field output variable within a cohesive interface layer. The peak stresses occur in the compressive region next to the notch as well as surrounding the entire notch due to the geometric discontinuity. Upon first inspection this distribution mirrors very closely the stress induced within the aluminum and the glass layers since it is the cohesive interface which transmits stress to the adjacent layers via shear. For the purposes of damage measurement Figure 5.47 through Figure 5.49 depict the distribution of the QUADECRT field output variable within the cohesive layers, which is a direct measurement of the amount of delamination within the interface through the satisfaction of the quadratic nominal strain criterion given in Equation (4.1).

An examination of the pattern of damage shown in Figure 5.47 through Figure 5.49 reveals that the compressive region, identified earlier as the region of interest for the YDB mechanism, is the region damaged as a result of the pin bearing load. Recall from Chapter 2 in the discussion and analytical calculation of the dimensions of the laterally unsupported glass layers subject to buckling that the delaminated portion was estimated to be equal to the calculated modified CCD distance. 


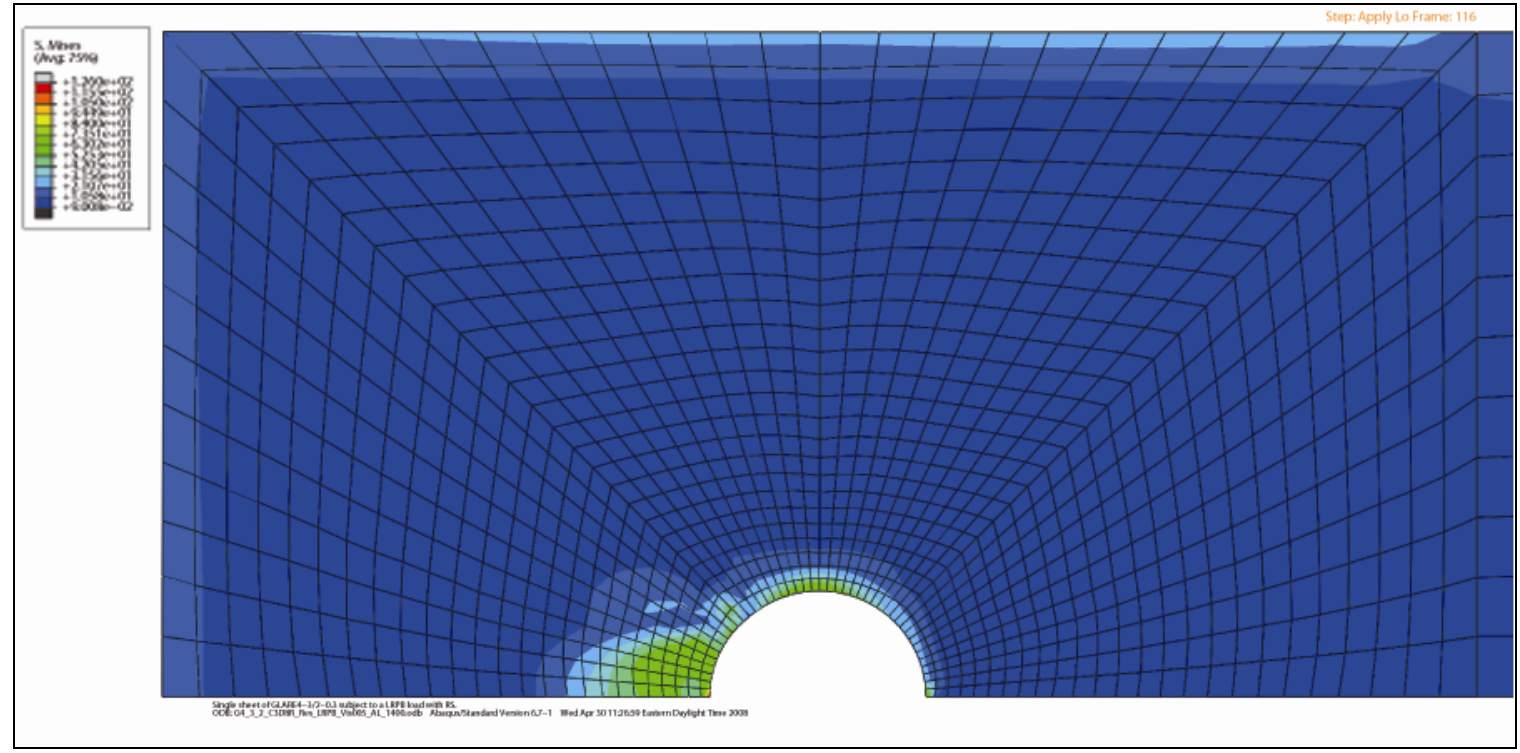

Figure 5.46: Typical distribution of the Von Mises field output variable in a cohesive interface layer of a GLARE4-3/2-0.3 variant.

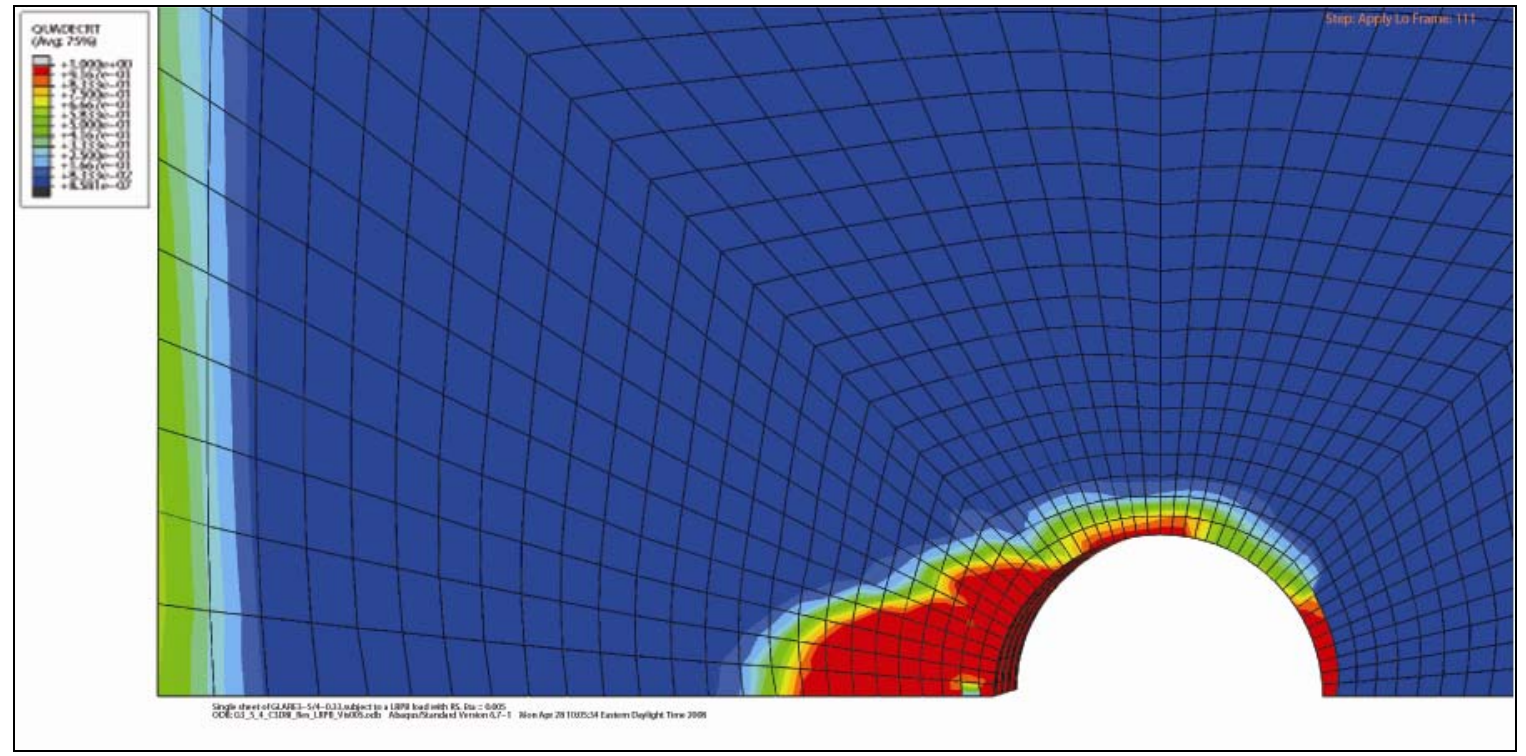

Figure 5.47: Distribution of delamination in the cohesive interface layer of a GLARE3-5/4-0.3 variant. 


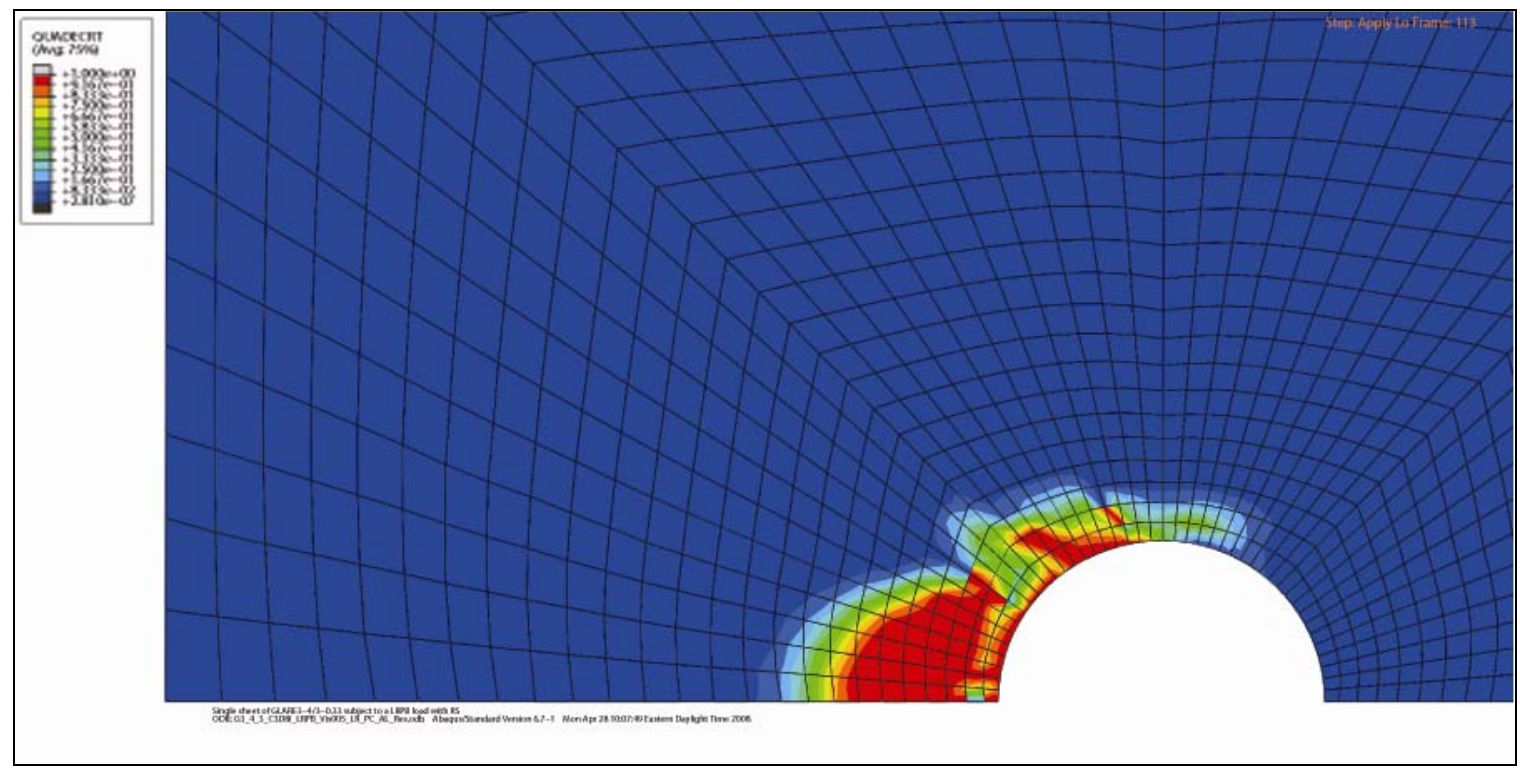

Figure 5.48: Distribution of delamination in the cohesive interface layer of a GLARE3-4/3-0.3 variant.

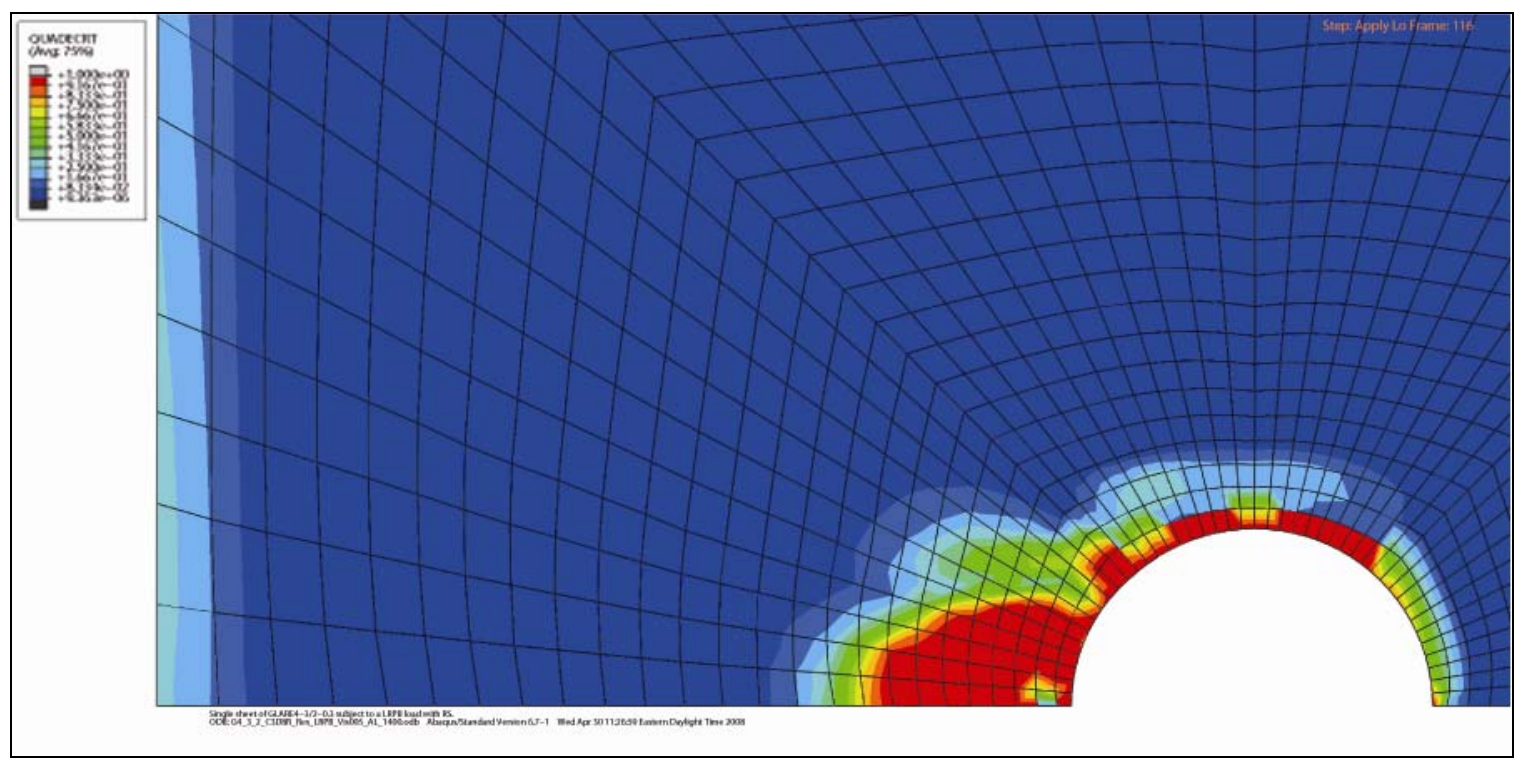

Figure 5.49: Distribution of delamination in the cohesive interface layer of a GLARE4-3/2-0.3 variant. 
This equality in distance is the link between buckling and delamination, both of which as defined are dependent upon the yielding of aluminum.

Therefore, a measurement of the delaminated distance within the compressive region, in one dimension moving away from the fastener hole as shown in Figure 5.50, can be used in comparison to the calculated modified CCD as well as measurements of delamination from SEM imagery.

The procedure for extracting the delaminated distance along a user defined nodal path is identical to the procedure employed while calculating the distance to the nominal values of ACYIELD, PE and PEEQ as performed on the aluminum layers. The threshold value between damage/undamaged for the QUADECRT field output variable which varies between 0 and 1 much in the same fashion as the ACYIELD variable was set to $2.5 \%$ of the maximum.. Values of QUADECRT were extracted from integration points along the plotted nodal path and were averaged for all the layers in the laminate to give an overall measure of delamination. These averaged values were then interpolated through a minimization of least squares error, yielding a function of damage versus distance as shown in Figure 5.51 to Figure 5.53.

An examination of these through the thickness averaged interpolation functions in Figure 5.51 to Figure 5.53 reveals a slight variation rather than a monotonic decay from maximum to minimum value. This is due in part to the averaging scheme employed which incorporated influence from all the interfaces layers in the laminate, some of which incurred slightly more or slightly less damage at each particular point from which values were extracted. 


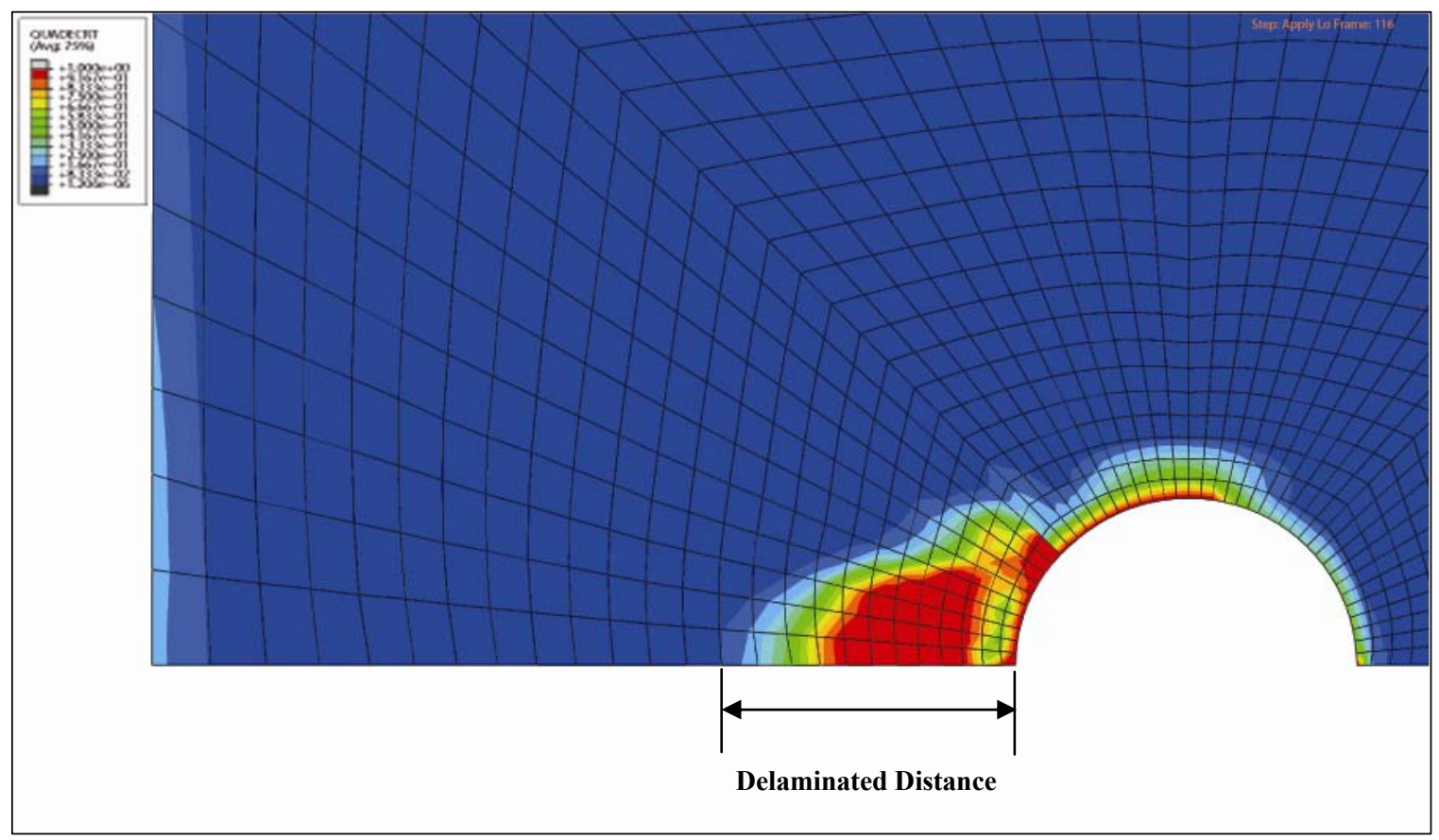

Figure 5.50: Schematic illustrating the measurement of delaminated distance within a cohesive interface layer.

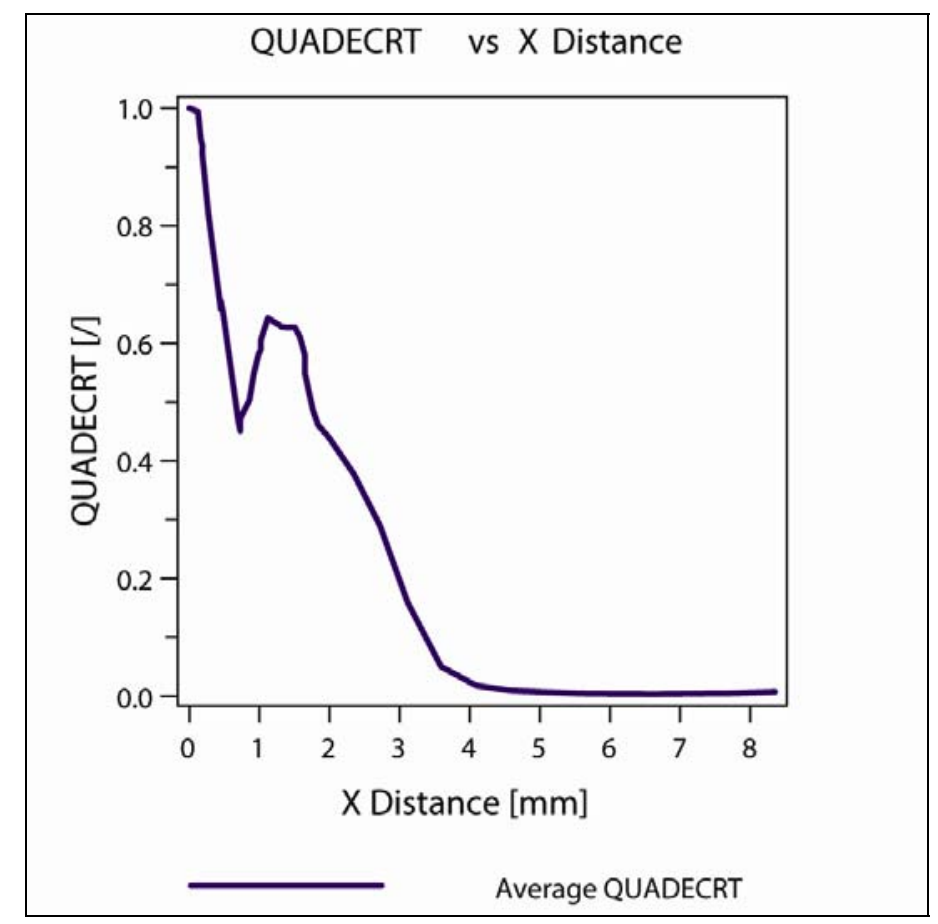

Figure 5.51: Plot of delamination damage versus increasing distance from fastener hole for a GLARE3-5/4-0.3 variant. 


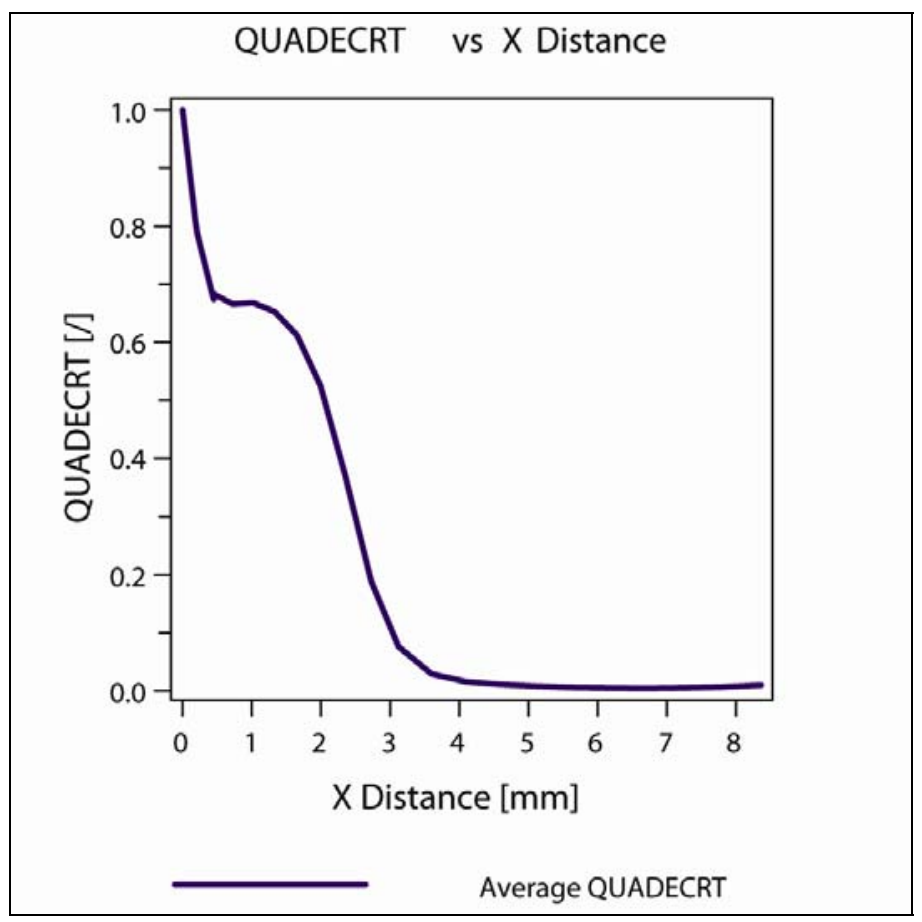

Figure 5.52: Plot of delamination damage versus increasing distance from fastener hole for a GLARE3-4/3-0.3 variant.

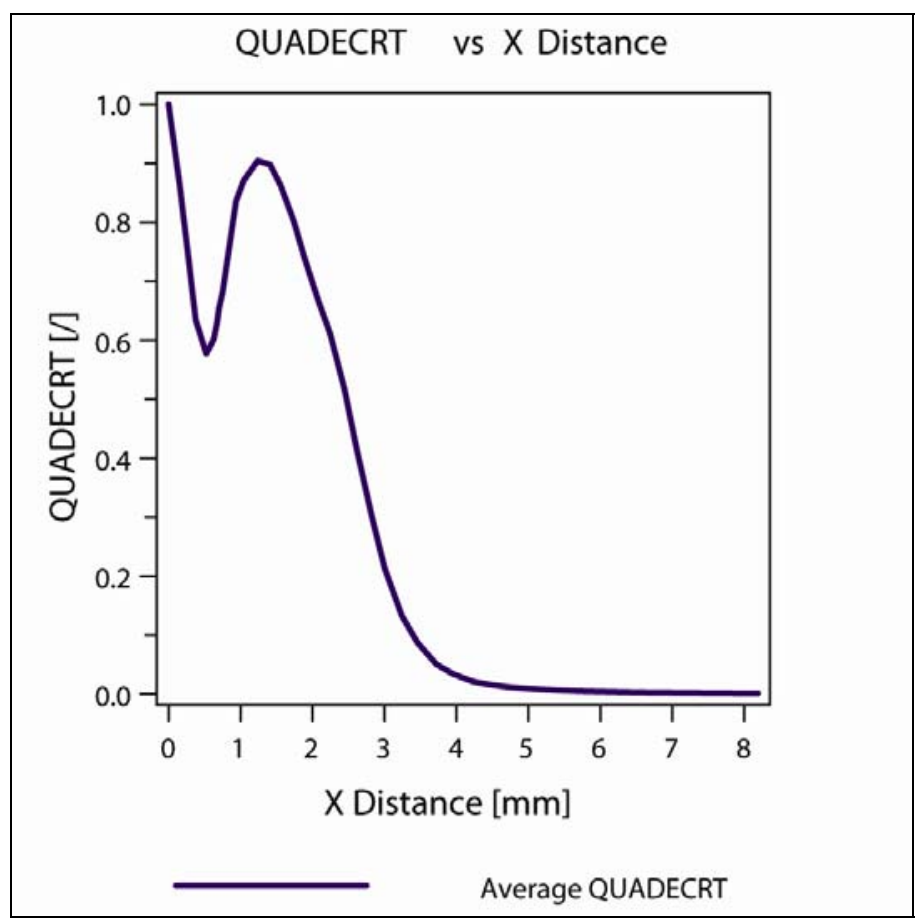

Figure 5.53: Plot of delamination damage versus increasing distance from fastener hole for a GLARE4-3/2-0.3 variant. 
These interpolated functions can be solved for the $2.5 \%$ minimum value yielding a precise measurement of the distance corresponding to nominal, or in this case, the cessation of damage/delamination. Table 5.10 summarizes the results of the extraction, averaging, interpolation and finally calculation of the delaminated distance for all the GLARE variants analyzed and compares their value to the modified CCD calculation. An examination of the values in Table 5.10 indicates that there is a close coupling between the modified CCD prediction and the delamination damage distance. This is precisely what was predicted by the proposed YDB mechanism which stipulates yielding, buckling and delamination all occurring within the calculated modified CCD distance. This very small error is certainly in support of the proposed notion and YDB mechanism, but empirical measurements must also provide support, in addition to analytical similarities.

Table 5.10: Summary of the average delamination damage distance compared to the variant specific modified CCD calculation.

\begin{tabular}{ccc}
\hline GLARE Variant & $\begin{array}{c}\text { Average Distance to } \\
\text { Nominal QUADECRT Value } \\
\text { [mm] }\end{array}$ & $\begin{array}{c}\text { Relative Error from } \\
\text { Predicted Modified CCD [\%] }\end{array}$ \\
\hline GLARE3-5/4-0.3 & 3.975 & -2.74 \\
\hline GLARE3-4/3-0.3 & 3.761 & 3.32 \\
\hline GLARE4-3/2-0.3 & 4.081 & -5.26 \\
\hline
\end{tabular}

\subsection{SEM Imagery of Failed Specimens and Comparison to Progressive Damage FE Model}

Images and measurements of the delaminated regions of failed specimens from the experimental portions of this study were examined from a cross sectional perspective 
under low vacuum in a JEOL JSM-6380LV scanning electron microscope (JEOL USA, Inc, Peabody, MA). Specimen preparation included sectioning the specimens with a water cooled diamond wafering saw at low speed (approximately $60 \mathrm{rpm}$ ) and mounting within a cold setting Lecoset 7007 mounting kit (Leco Instruments Ltd., Mississauga, ON, CA). Surface preparation was done using an automated polisher capable of polishing 6 specimens simultaneously with 180 grit, 600 grit, 1200 grit silica carbide paper followed by polishing with 5 micron alumina (aluminum oxide) particles on a cloth substrate with water as a coolant. Polishing continued until a mirror finish was achieved on the exposed aluminum faces of the cross-sectioned laminate. Care had to be exercised in setting the pressure for polishing to avoid any fibre pullout as a result of the abrading and polishing.

Figure 5.54 through Figure 5.57 show some of the SEM images taken for each variant tested and include the measurement of total delamination. The imaged area was the cross section of material directly adjacent to the fastener hole on its bearing surface.

Measurements of delamination were taken from the furthest portion of aluminum to the end of observable damage. The furthest portion of aluminum was chosen since it exhibited a flattened edge, visibly induced through contact with the pin. These landmarks were reliably found due to their striking appearance and represent a consistent means of establishing a benchmark zero for delamination measurement. 


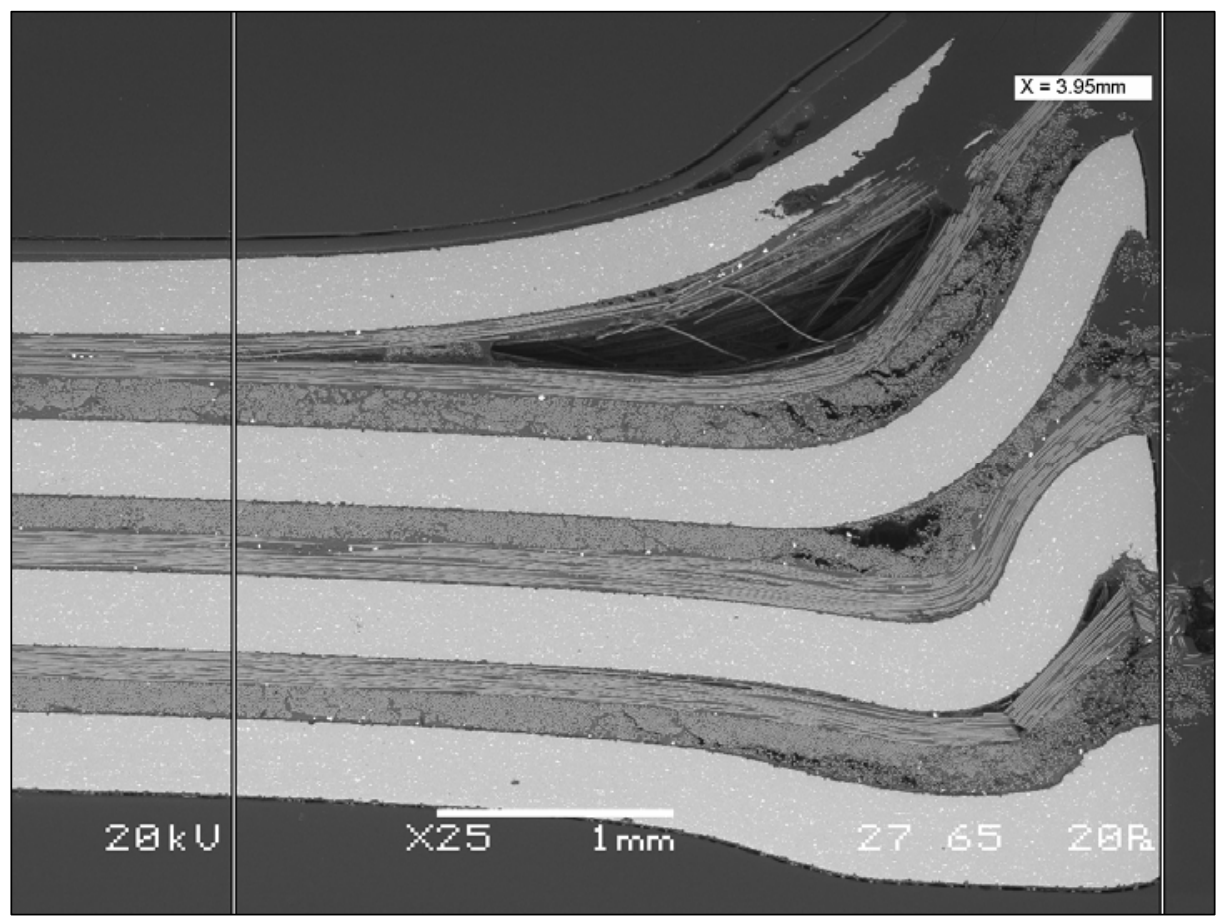

Figure 5.54: SEM image of a GLARE3-4/3-0.3 variant with delamination measurement.

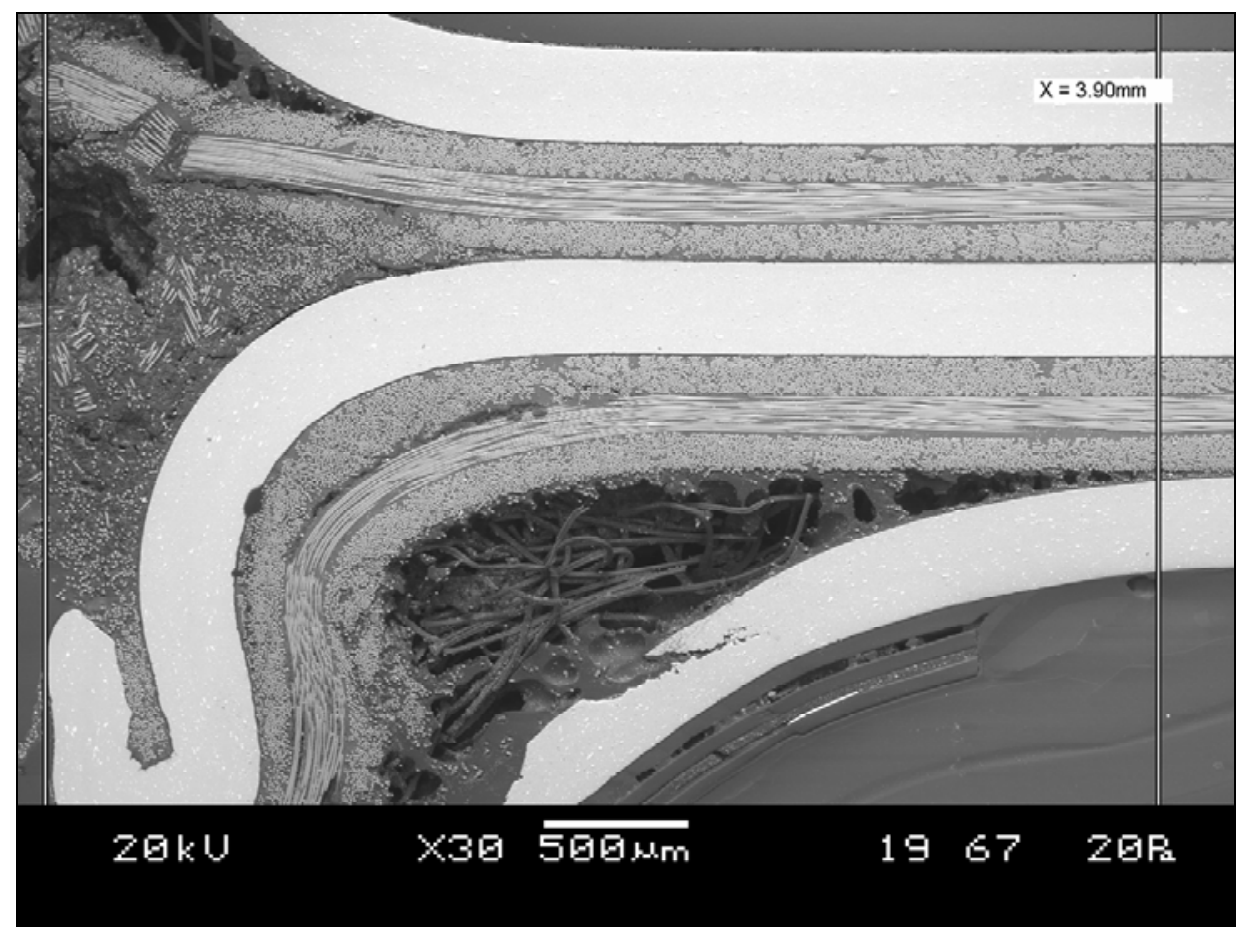

Figure 5.55: SEM image of a GLARE4-3/2-0.3 variant with delamination measurement. 


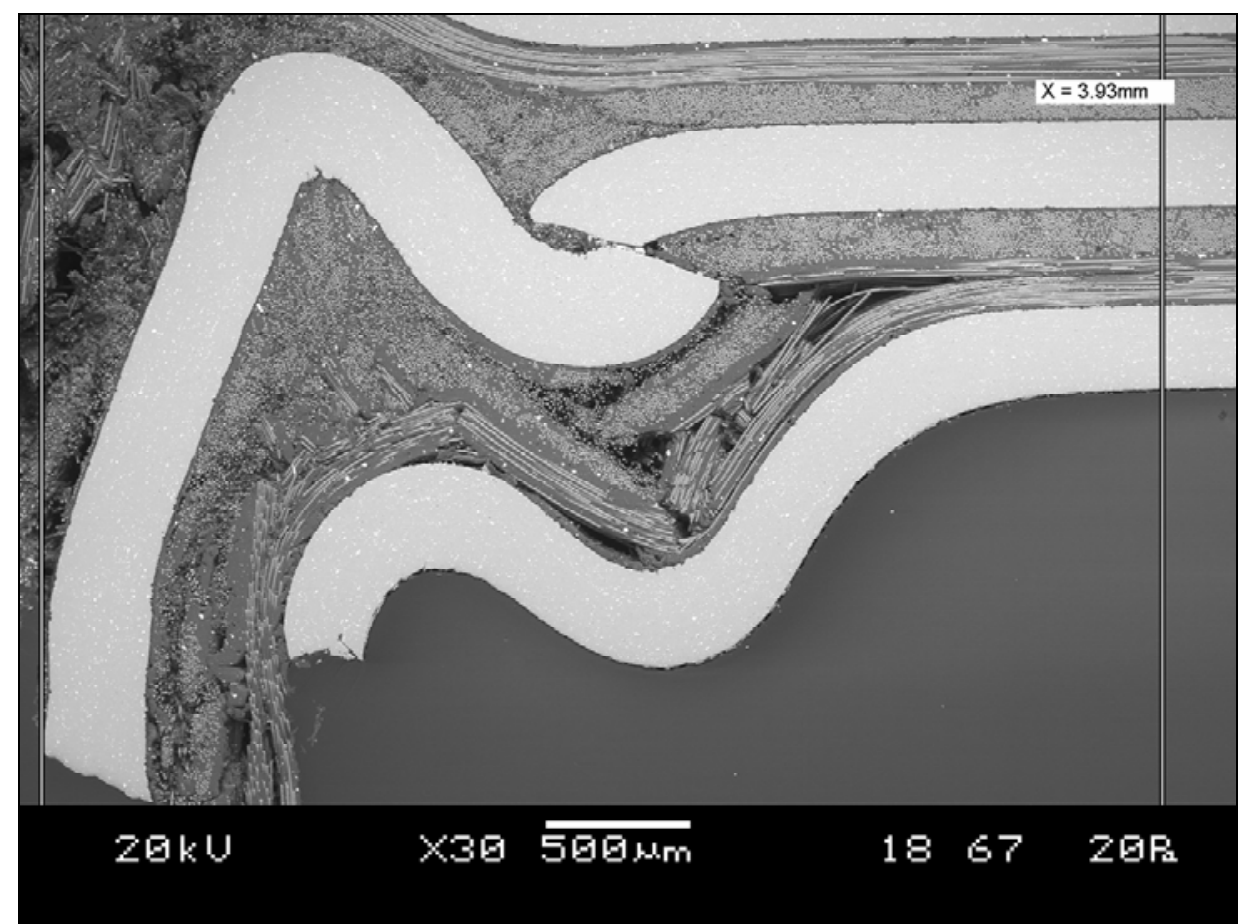

Figure 5.56: SEM image of a GLARE3-4/3-0.3 variant with delamination measurement.

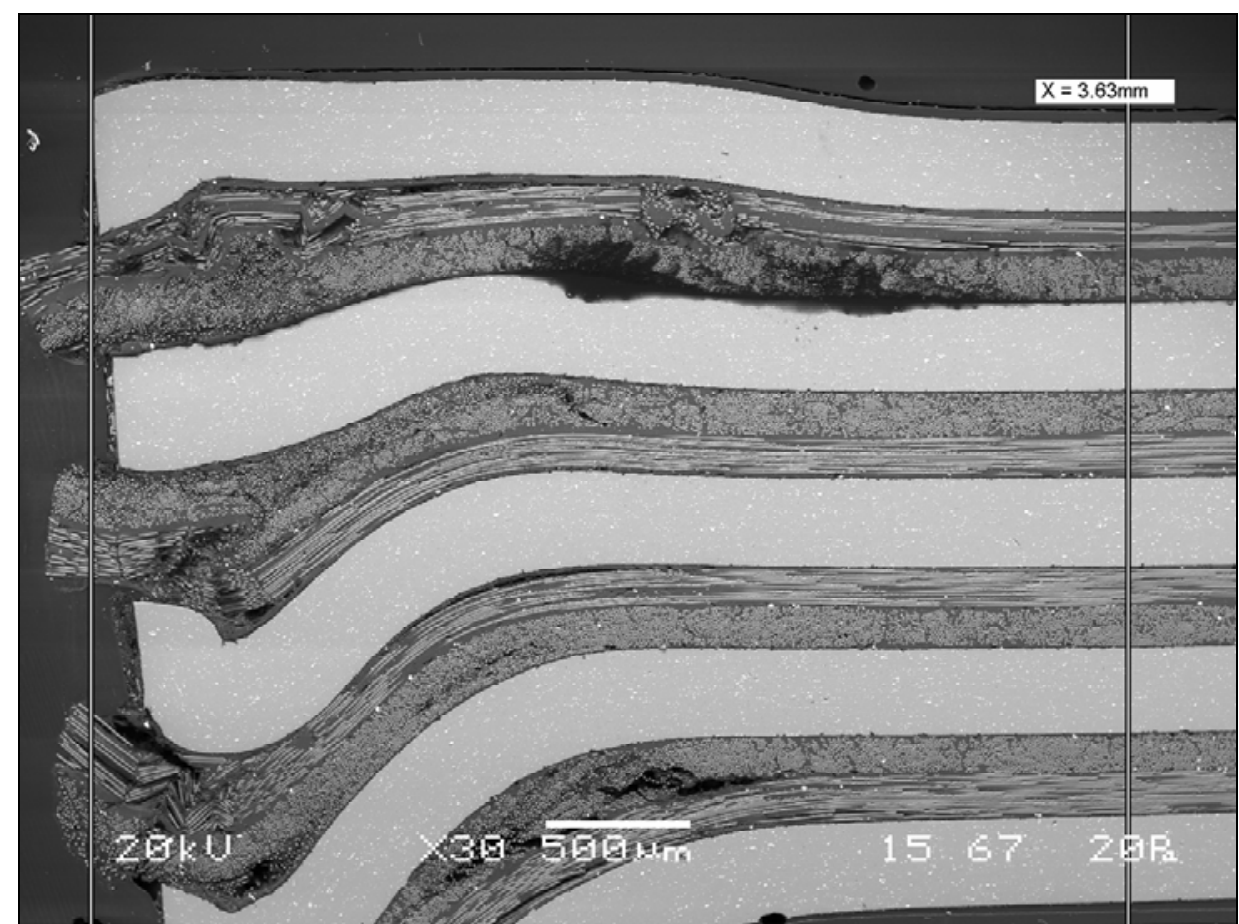

Figure 5.57: SEM image of a GLARE3-5/4-0.3 variant with delamination measurement. 
At the other end of the measurement, the damage zone end presented an increased challenge since defining an end point required multiple images each with increased magnification until a clear and defined end of damage was visible. This was found by examining the entire imaged area and noting the region of maximum damage. Figure 5.58 through Figure 5.60 depict some of the typical damage zone end points with a characteristic tapering off of damage analogous to the tapering off exhibited by the progressive damage FE results.

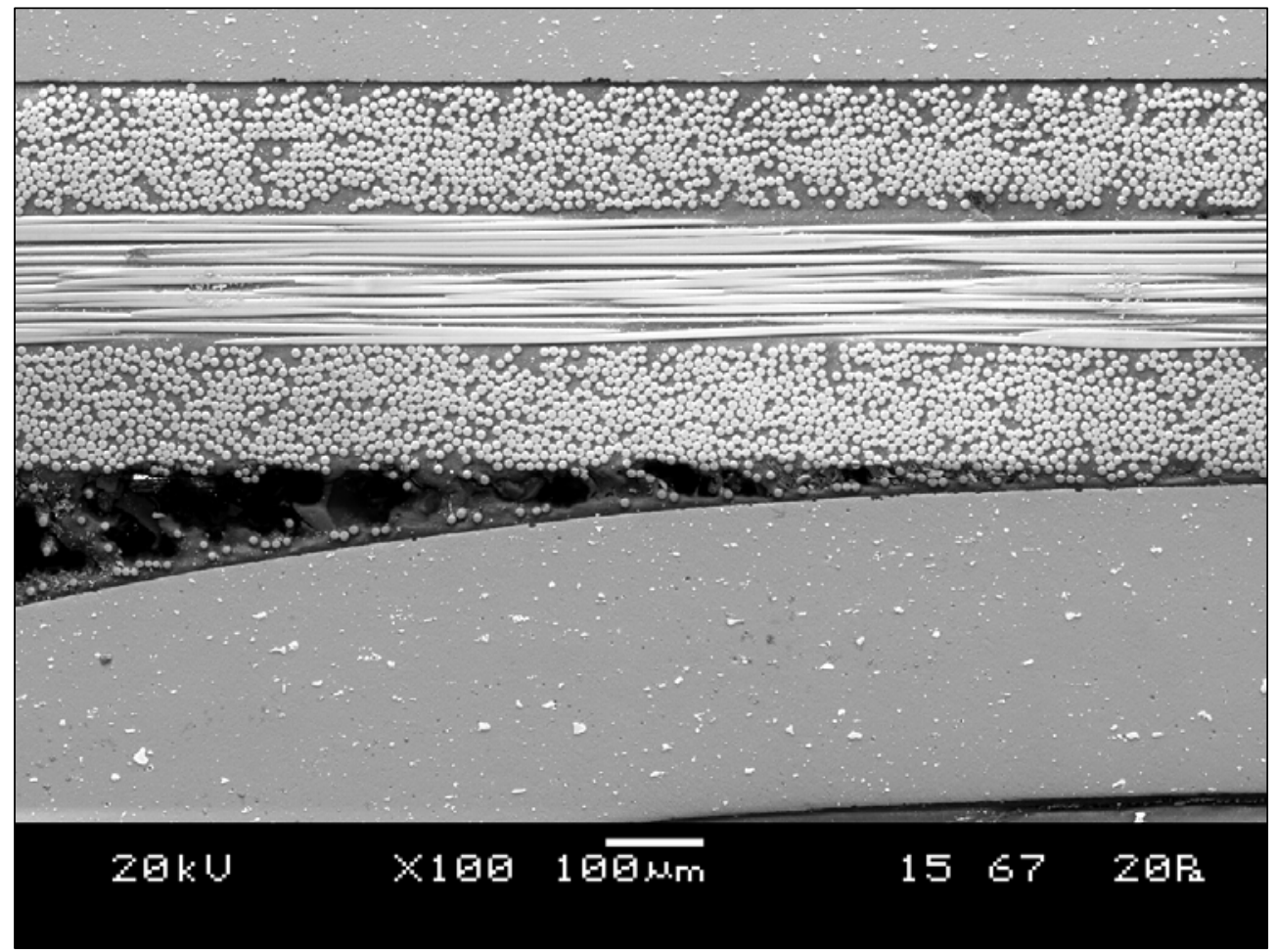

Figure 5.58: End point of delamination damage within a GLARE4-3/2-0.3 variant. 


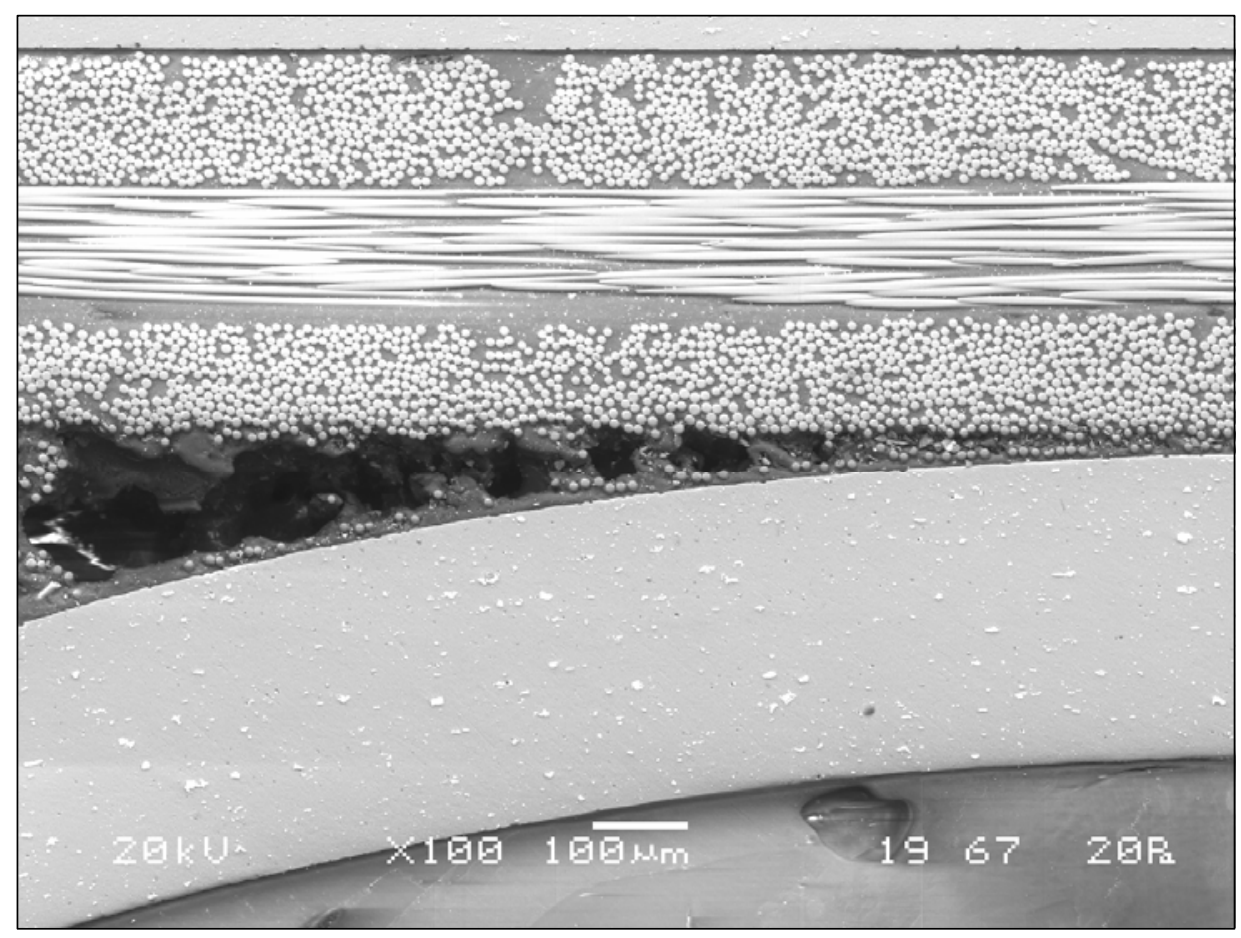

Figure 5.59: End point of delamination damage within a GLARE4-3/2-0.3 variant.

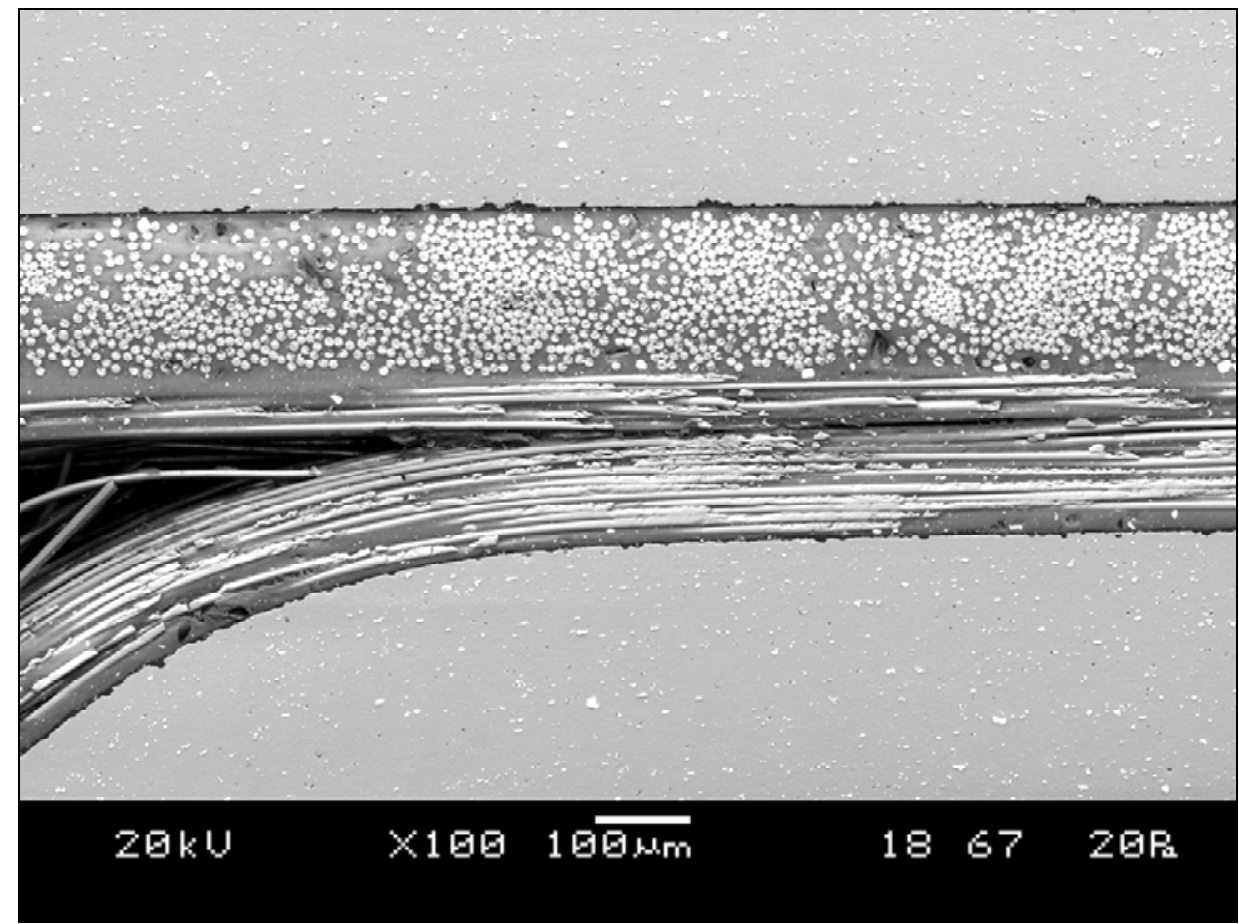

Figure 5.60: End point of delamination damage within a GLARE3-4/3-0.3 variant. 
Figure 5.61 shows a region of delamination damage with increased magnification to highlight the separation between adjacent layers which in this case is delamination between the aluminum and a transversely oriented glass prepreg layer. It is noteworthy to comment on the damage modes visible in the micrograph.

Both mode I and mode II damage is clearly visible indicating that the mixed-mode delamination approach employed in the delamination modeling portion of the FE analysis correctly assumed a mixed-mode scenario.

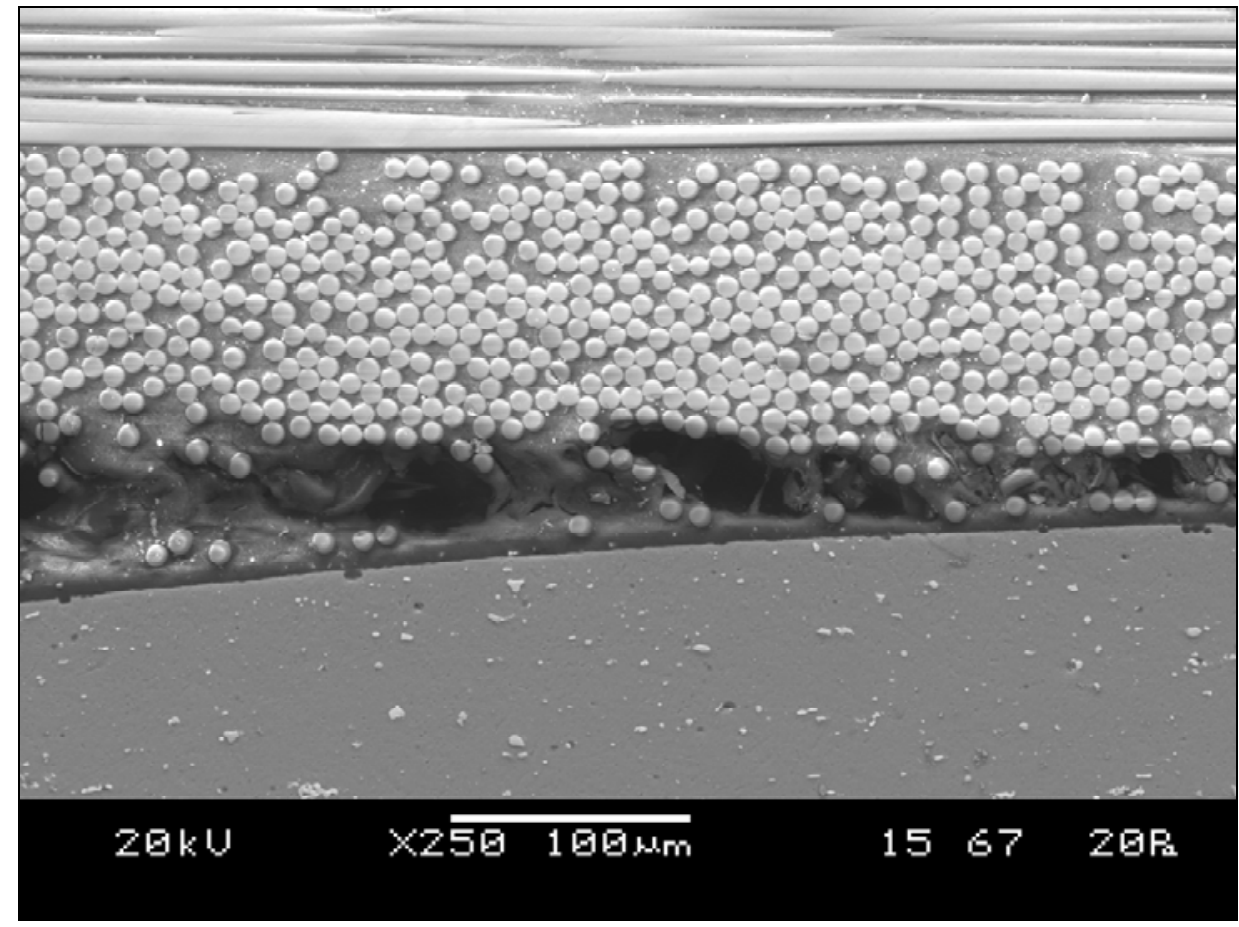

Figure 5.61: Highly magnified view of a delaminated portion within a typical GLARE variant. Note the combination of pulling and shearing, characteristic of mixed-mode damage.

Four specimens from each variant were prepared and imaged for a total of twelve images, producing a representative portion of the total number of specimens tested. Table 5.11 
summarizes the results of the delamination measurement for each specimen variant, their average delamination distance and some associated statistics.

Here we see that the measured values show a very small scatter, suggesting that the definitions employed for the boundaries of measurement were robust and produced consistent values. Bearing the aforementioned measurements in mind, Table 5.12 presents these values alongside the predicted delamination distances from the progressive damage FE modeling. Clearly the results in Table 5.12 indicate a strong correlation between the predicted values and their measured counterparts with a small relative error. The implication of these results, coupled with those presented earlier in the chapter, is that the developed progressive damage FE model accurately simulates the bearing behaviour of GLARE FMLs, including their failure.

Table 5.11: Summary of the measured delaminated distances and some associated statistics.

\begin{tabular}{|c|c|c|c|c|c|}
\hline $\begin{array}{l}\text { GLARE } \\
\text { Variant }\end{array}$ & Specimen & $\begin{array}{c}\text { Measured } \\
\text { Delam. Dist. } \\
\text { [mm] }\end{array}$ & $\begin{array}{c}\text { Average } \\
\text { Delam. Dist. } \\
\text { [mm] }\end{array}$ & $\begin{array}{c}\text { Standard } \\
\text { Deviation } \\
\text { [mm] }\end{array}$ & $\begin{array}{c}\text { Coefficient } \\
\text { of Variation } \\
{[\%]}\end{array}$ \\
\hline \multirow{4}{*}{$\begin{array}{c}\text { GLARE3- } \\
5 / 4-0.3\end{array}$} & S01 & 3.630 & \multirow{4}{*}{3.882} & \multirow{4}{*}{0.161} & \multirow{4}{*}{4.09} \\
\hline & S09 & 3.840 & & & \\
\hline & S10 & 3.850 & & & \\
\hline & S11 & 4.210 & & & \\
\hline \multirow{4}{*}{$\begin{array}{c}\text { GLARE3- } \\
\text { 4/3-0.3 }\end{array}$} & S04 & 3.745 & \multirow{4}{*}{3.941} & \multirow{4}{*}{0.240} & \multirow{4}{*}{6.20} \\
\hline & S09 & 3.950 & & & \\
\hline & S10 & 4.140 & & & \\
\hline & S11 & 3.930 & & & \\
\hline \multirow{4}{*}{$\begin{array}{c}\text { GLARE4- } \\
3 / 2-0.3\end{array}$} & S03 & 4.160 & \multirow{4}{*}{4.027} & \multirow{4}{*}{0.293} & \multirow{4}{*}{7.28} \\
\hline & S04 & 3.690 & & & \\
\hline & S05 & 4.360 & & & \\
\hline & S06 & 3.900 & & & \\
\hline
\end{tabular}


Table 5.12: A comparison of the predicted delaminated distances as per the $\mathrm{FE}$ model and the measured delaminated distances from the SEM imagery.

\begin{tabular}{cccc}
\hline GLARE Variant & $\begin{array}{c}\text { Predicted } \\
\text { Delamination } \\
\text { Distance [mm] }\end{array}$ & $\begin{array}{c}\text { Measured } \\
\text { Delamination } \\
\text { Distance [mm] }\end{array}$ & Relative Error [\%] \\
\hline GLARE3-5/4-0.3 & 3.975 & 3.882 & 3.97 \\
\hline GLARE3-4/3-0.3 & 3.761 & 3.941 & -4.55 \\
\hline GLARE4-3/2-0.3 & 4.081 & 4.027 & 1.33 \\
\hline
\end{tabular}

Equally as important, these results represent an experimental investigation of the connection between delamination and the YDB mechanism. Previously it was experimentally shown that yielding and buckling are connected to the modified CCD.

The results of Table 5.12 go on to show that there is a connection between delamination and the modified CCD as well, through the YDB mechanism.

The proposition of yielding coupled to delamination and buckling as outlined in the YDB mechanism has been experimentally investigated. The outcomes suggest that the laminate is dependent upon one of its constituents and one parameter within that constituent; that of the yield strength of the aluminum. The modeling and experimental portions of this study represent a practical damage prediction methodology for the bearing strength of GLARE FMLs. The strong correlation between the progressive damage FE model and the experiments suggest that future analyses can be conducted with this approach, making it a valuable design tool. This was the intention of the study. 


\subsection{Chapter Summary}

In this chapter the results of the residual stress and applied load step in the progressive damage FE models were presented. The results from the FE analyses show that minor delamination damage occurred near geometric discontinuities of the laminate even before any mechanical loads were applied during the simulation. The results of the applied load produced stress distributions and damage similar to the experimental study. Examinations of the requested field output variables enabled a comparison to experimental values and showed small relative errors. SEM imagery of failed specimens allowed for a comparison of delamination damage to the predicted damage of the FE model with good results. The results of the comparison suggest a connection between delamination to yielding and buckling, as per the modified CCD and YDB mechanism. 


\section{Chapter Six: Conclusions}

The scope of this research included extensive testing of GLARE FMLs in a pin bearing loading configuration at the critical design ratio of $\mathrm{E} / \mathrm{D}=3$. In addition, an extension of the characteristic dimension approach, a failure mechanism, material characterization and definition of yield strength were proposed, with comparisons to the empirical investigations. These developments represent a practical methodology for simulating the mechanical behaviour and ultimate failure of GLARE FMLs loaded in pin bearing. It was used to examine the yielding and delamination behaviour alongside analytical and experimental investigations of buckling to probe the implications of the proposed YDB mechanism. The progressive damage FE model created in conjunction with the modified CCD, YDB mechanism and yield strength definition provides a valuable toolbox for the design of future FML applications and can be used for improved mechanical fastening qualification. As a result of the research discussed herein, the following conclusions can be drawn:

1. A redefinition of the compressive characteristic dimension based upon one constituent and one parameter within that constituent allows for the extension of the characteristic dimension approach to as FMLs as well. 
2. The orthotropic plate buckling analysis of the laterally unsupported regions of the glass prepreg layers revealed small values of critical buckling stress when compared to the yield stress of the aluminum. This suggests that the contribution of the glass prepreg layers to the overall bearing strength of the laminate, in a bearing failure mode, can be considered negligible and the bearing strength of the joint is governed by the coupling of aluminum yielding with delamination buckling as per the YDB mechanism.

3. Pin bearing experiments performed on both quasi-isotropic and orthotropic variants of GLARE employed an approach that measured strain both locally and globally. This enabled the examination of deformation, yield and buckling near the modified CCD in addition to standardized measurements.

4. The proposed piecewise characterization of the stress-strain curves for GLARE and bisection protocol definition of yield were successfully applied without adjustments to both quasi-isotropic and orthotropic variants of GLARE. These techniques were also applied to standard tensile testing of GLARE with equal success.

5. The developed progressive damage FE models including residual stress, successfully simulated the pin bearing case for FMLs. Comparison to experimental values showed small relative errors and strong connections between yielding, delamination and buckling as per the YDB mechanism, suggesting it is the governing mechanism of failure.

6. The presented methodology can be used as a practical damage prediction methodology for the pin bearing behaviour of GLARE FMLs as part of the design process. 


\section{Chapter Seven: Future Considerations}

The research presented included experimental, analytical and numerical considerations as part of a practical damage prediction methodology for the pin bearing behaviour of GLARE and FMLs in general. The traditional application of monolithic alloys has given way to increased use of composite materials, specifically in the aerospace sector where demand for higher performance materials is at its apex. FMLs as a new material for application to aircraft brings the best of both worlds and there remains considerable work to be done before a complete set of tools is available to the designer when implementing them in future applications.

\subsection{Experimental Considerations}

Newer instrumentation technologies including optical fibres with laser etched Fibre Bragg Gratings (FBGs) represent the ability to embed sensory instrumentation within the laminate to allow for an internal examination in addition to surface based approaches. Data collected from FBGs would be insensitive to electromagnetic interference by virtue of their optical nature and could include measurements of moisture and temperature. With increased sensitivity, the process of delamination could be measured both from a mechanical point of view and a thermodynamic one allowing for additional modeling and 
conclusions to be drawn. The dramatically higher cost of these sensors and incomplete understanding of the effect of their embedment makes their application somewhat limited at the current time.

\subsection{Modeling Considerations}

Scaling up of the modeling could be taken to the extreme including entire fuselage panels and their joints. Full scale universal testing machines could be modeled to investigate the losses due to alignment, hydraulic grips, fixtures and pin seating. Modeling such as this could provide additional insight, however, it is not terribly practical since it is extremely time and computationally expensive. 


\section{References}

1. Vermeeren, C.A.J.R. (2003). An Historic Overview of the Development of Fibre Metal Laminates, Applied Composite Materials, 10: 189-205.

2. Bijlmer, P.F.A. (1978). Fracture Toughness of Multiply Layer Adhesive Bonded Aluminum Alloy Sheet, Proceedings of the International Congress of the Aeronautical Sciences, Lisbon, Portugal: 544-554.

3. Kaufman, J.G. (1967). Fracture Toughness of 7075-T6 and -T651 Sheet, Plate and Multi-Layered Adhesive Bonded Panels, Transactions of ASME, Journal of Basic Engineering, 89: 303-307.

4. Johnson, W.J. (1983). Damage Tolerance Evaluation of Adhesively Laminated Titanium, Journal of Engineering Materials and Technology, 105: 182-187.

5. Alic, J.A. and Chang, H. (1975). Comparison of Fracture and Fatigue Properties of Clad 7075-T6 Aluminum in Monolithic and Laminated Forms, SAE Paper 750511.

6. Johnson, W.S. and Stratton, J.M. (1977). Effect of Remote Stresses and Stress Intensity Factors for an Adhesively Bonded Multi-ply Laminate, Journal of Engineering Fracture Mechanics, 9: 411-421.

7. Ratwani, M.M. (1979). Analysis of Cracked, Adhesively Bonded Laminated Structures, AIAA Journal, 17(9): 988-994.

8. Broek, D. (1986). Elementary Engineering Fracture Mechanics, Kluwer Academic Publishers, Hingham, MA, USA. 
9. Sandford, R.J. (2003). Principles of Fracture Mechanics, Pearson Education., Upper Saddle River, NJ, USA.

10. Dowling, N.E. (1993). Mechanical Behaviour of Materials, Prentice Hall, Englewood Cliffs, NJ, USA.

11. Miller, J.L, Progar, D.J., Johnson, W.S., St. Clair, T.L. (1994). Preliminary Evaluation of Hybrid Titanium Composite Laminates, NASA-TM-109095.

12. Volgelsang, L.B., Marissen, R., Shijve, J. (1981). A New Fatigue Resistant Material: Aramid Reinforced Aluminum Laminate (ARALL), Proceedings of the $11^{\text {th }}$ ICAF Symposium, Noordwijkerhout, the Netherlands.

13. Marissen, R. and Vogelsang, L.B. (1981). Development of a New Hybrid Material: 'ARALL', Intercontinental SAMPE Meeting, Cannes, France.

14. Gunnink, J.W., Vogelsang, L.B., Schijve, J. (1982). Application of a New Hybrid Material (ARALL) in Aircraft Structures, Proceedings of the $13^{\text {th }}$ Congress of ICAS, Seattle, WA, USA: 990-1000.

15. Vogelsang, L.B. and Gunnink, J.W. (1986). ARALL: A Material's Challenge for the Next Generation of Aircraft, Materials and Design, 7: 287-300.

16. Bucci, R.J., Mueller, L.N., Vogelsang, L.B., Gunnink, J.W. (1989). ARALL Laminates, Treatise on Materials Science and Technology, 31: 295-321.

17. Vlot A. (author), Vlot, A \& Gunnick, J.W. (eds.) (2001). Historic Overview, Fibre Metal Laminates an Introduction, Ch.1, Kluwer Academic Publishers, Dordrecht, Netherlands. 
18. Roebroeks, G. (1991). Towards Glare - The Development of a Fatigue Insensitive and Damage Tolerant Aircraft Material, Dissertation - Delft University of Technology.

19. Young, J.B. Landry, J.G.N. \& Cavoulacos, V.N. (1994). Crack Growth and Residual Strength Characteristics of Two Grades of Glass-Reinforced Aluminum 'Glare', Composite Structures, 27: 457-469.

20. Gunnink, J.W., Vlot, A., De Vries, T.J., Van Der Hoeven, W. (2002). Glare Technology Development 1997-2000, Applied Composite Materials, 9: 201-219.

21. Wu, G. and Yang, J.M. (2005). The Mechanical Behaviour of GLARE Laminates for Aircraft Structures, Journal of the Minerals, Metals and Materials Society, 57(1): 72-80.

22. Armstrong, K.B., and Barrett, R.T. (1998). Care and Repair of Advanced Composites, Society of Automotive Engineers, Warrendale, PA, USA.

23. Ritchie, R.O., Yu, W., Bucci, R.J. (1989). Fatigue Crack Propagation in ARALL Laminates: Measurement of the Effect of Crack-Tip Shielding from Crack Bridging, Engineering Fracture Mechanics, 32: 361-377.

24. Schijve, J. (1994). Fatigue of Aircraft materials and Structures, Fatigue, 16(1): 21-32.

25. Cudzilo, B.E. and Tan, C.L. (2003). Numerical Fracture Mechanics Analysis of Cracked Fibre Metal Laminates with Cut-Outs, Electronic Journal of Boundary Elements, 1(3): 336-403. 
26. Roebroeks, G.H.J.J. (author), Vlot, A. and Gunnink, J.W. (eds.) (2001). Glare Features, Fibre Metal Laminates an Introduction, 25, Kluwer Academic Publishers, Dordrecht, the Netherlands.

27. Hagenbeek, M., Van Hengel, C., Bosker, O.J., Vermeeren, C.A.J.R. (2003). Static Properties of Fibre Metal Laminates, Applied Composite Materials, 10: 207-222. 28. Asundi, A. and Choi, Alta, Y.N. (1997). Fiber Metal Laminates: An Advanced Material for Future Aircraft, Journal of Materials Processing Technology, 63: 384-394.

29. Department of Defense (1998). Metallic Materials and Elements for Aerospace Vehicles Structures, $M I L-H D B K-5 H$.

30. Verolme, J.L. (1996). Prediction of Stress-Strain Curves of GLARE, Structural Laminates Company, Technical Report TD-R-96-004, Delft, the Netherlands.

31. Van Rijn, J.C.F.N. (2000). A Calculation Method for the Stress-Strain Curves of GLARE 3 and GLARE 4B, National Aerospace Laboratory, Report NLR-CR2000-172, Amsterdam, the Netherlands.

32. Wu, H.F. (1994). Use of the Rule of Mixtures and Metal Volume Fraction for Mechanical Property Predictions of Fiber-Reinforced Aluminum Laminates, Journal of Material Science, 29: 4583-4591.

33. Ipjma, M.S. (author), Vlot, A., and Gunnink, J.W. (eds.) (2001). Material Design Allowables and Qualification, Fibre Metal Laminates an Introduction, 69, Kluwer Academic Publishers, Dordrecht, the Netherlands.

34. Cook, J. and Donnellan, M.E. (1991). Tensile and Interlaminar Properties of GLARE (Trade Name) Laminates, Technical Report NADC-91087-60, Naval Air 
Development Centre Air Vehicle and Crew Systems Technology Department, Westminster, PA, USA.

35. Alderliesten, R.C. (author), Vlot, A \& Gunnick, J.W. (eds.) (2001). Fatigue, Fibre Metal Laminates an Introduction, 255, Kluwer Academic Publishers, Dordrecht, Netherlands.

36. Alderliesten, R.C., Hagenbeek, M. Homan, J.J., Hooijmeijer, P.A., De Vries, T.J., Vermeeren, C.A.J.R. (2003). Fatigue and Damage Tolerance of Glare, Applied Composite Materials, 10: 223-242.

37. Vasek, A., Polak, J., Kozak, V. (1997). Fatigue Crack Initiation in Fibre-Metal Laminate GLARE 2, Materials Science and Engineering, A234-236: 621-624.

38. Takamatsu, T., Matsamura, T., Ogura, N., Shimokawa, T., Kakuta, Y. (1999). Fatigue Crack Growth Properties of a GLARE 3-5/4 Fibre/Metal Laminate, Engineering Fracture Mechanics, 63: 253-272.

39. Shim, D.J., Alderliesten, R.C., Spearing, S.M., Burianek, D.A. (2003). Fatigue Crack Growth Prediction in GLARE Hybrid Laminates, Composites Science and Technology, 63: 1759-1767.

40. Roebroeks, G.H.J.J. (1994). Fibre-Metal Laminates: Recent Developments and Applications, Fatigue, 16: 33-42.

41. Laliberté, J.F., Poon, C., Straznicky, P.V., Fahr, A. (2002). Post-Impact Fatigue Damage Growth in Fibre Metal Laminates. International Journal of Fatigue, $\mathbf{2 4}$ (2-4): 249-256. 
42. Wu, X.R. and Guo, Y.J. (2002) Fatigue Behaviour and Life Prediction of Fibre Reinforced Metal Laminates Under Constant and Variable Amplitude Loading, Fatigue and Fracture of Engineering Materials and Structures, 25: 417-432.

43. De Vries, T.J., Vlot, A. (2001). The Influence of the Constituent Properties on the Residual Strength of GLARE, Applied Composite Materials, 8: 263-277.

44. Buemler, T. (author), Vlot, A. \& Gunnick, J.W. (eds.) (2001). Damage Tolerance Aspects, Fibre Metal Laminates an Introduction, 219, Kluwer Academic Publishers, Dordrecht, the Netherlands.

45. Bosker, O.J. (author), Vlot, A., and Gunnink, J.W. (eds.) (2001). Blunt Notch Strength, Fibre Metal Laminates an Introduction, 117, Kluwer Academic Publishers, Dordrecht, the Netherlands.

46. Hol, J. and Antonelli, V. (2003). Progressive Damage Modeling of FMLs Implementation in a UMAT Subroutine, Proceedings of the 2003 ABAQUS User's Conference, 1-10.

47. Chang, P.Y., Yang, J.M., Seo, H., Hahn, H.T. (2007). Off-Axis Fatigue Cracking Behaviour in Notched Fibre Metal Laminates, Fatigue and Fracture of Engineering Materials and Structures, 30: 1158-1171.

48. Hagenbeek, M. (author), Vlot, A., and Gunnink, J.W. (eds.) (2001). Impact Properties, Fibre Metal Laminates an Introduction, 409, Kluwer Academic Publishers, Dordrecht, the Netherlands.

49. Vlot, A. (1993). Impact Properties of Fibre Metal Laminates, Composites Engineering, 10: 911-927. 
50. Laliberté, J.F. (2002). Investigation of Low-Velocity Impact Damage in Fibre Metal Laminates, Dissertation - Carleton University.

51. Laliberté, J.F., Straznicky, P.V., Poon, C. (2005). Impact Damage in Fibre Metal Laminates, Part 1: Experiment. American Association of Aeronautics and Astronautics Journal, 43 (11): 2445-2543.

52. Dodd, D.C., Hall, C.T.M., Pollard, J., Snell, M.A. (1994). Burn Through Resistance of Fuselages: Initial Findings, Civil Aviation Authority CAA Paper 94002, London, U.K.

53. Roebroeks, G.H.J.J. (1996). GLARE: A Structural Material for Fire Resistant Fuselages, Aircraft Fire Safety, AGARD Conference Proceedings 587, 26: 1-13.

54. Hooijmeijer, P.A. (author), Vlot, A., and Gunnink, J.W. (eds.) (2001). Burn Through and Lightning Strike, Fibre Metal Laminates an Introduction, 399, Kluwer Academic Publishers, Dordrecht, the Netherlands.

55. Borgognje, B., Ijpma, M.S., ‘t Hart, W.G.J. (authors), Vlot, A., and Gunnink, J.W. (eds.) (2001). Corrosion, Fibre Metal Laminates an Introduction, 427, Kluwer Academic Publishers, Dordrecht, the Netherlands.

56. Frizzell, R.M., McCarthy, C.T., McCarthy, M.A. (2008). An Experimental Investigation into the Progressive Damage in Pin-Loaded Fibre Metal Laminates. Composites: Part B, 39:907:925.

57. van Rooijen, R.G.J. (2006). Bearing Strength Characteristics of Standard and Steel Reinforced GLARE, Dissertation - Delft University of Technology. 
58. van Rooijen, R.G.J., Sinke, J., De Vries, T.J., Van Der Zwaag, S. (2006). The Bearing Strength of Fibre Metal Laminates, Journal of Composite Materials, 40 (1): 5-19.

59. Slagter, W.J. (1994). Static Strength of Riveted Joints in Fibre Metal Laminates, Dissertation - Delft University of Technology.

60. Slagter, W.J. (1992). On The Bearing Strength of Fibre Metal Laminates, Journal of Composite Materials, 26 (17): 2543-2566.

61. Wu, H.F. and Slagter, W.J. (1994). Parametric Studies of Bearing Strength for Fiber/Metal Laminates, Journal of Aircraft, 31 (4): 936-945.

62. Caprino, G., Squillace, A., Giorleo, L., Nele, L., Rossi, L. (2005). Pin and Bolt Bearing Strength of Fibreglass/Aluminum Laminates. Composites: Part A, 36: $1307-1315$.

63. Meola, C., Squillace, A., Giorleo, G., Nele, L. (2003). Experimental Characterization of an Innovative Glare ${ }^{\circledR}$ Fibre Reinforced Metal Laminate in Pin Bearing, Journal of Composite Materials, 37 (17): 1543-1552.

64. Buemler, T. (2004). Flying Glare: A Contribution to Aircraft Certification Issues on Strength Properties in Non-Damaged and Damaged Glare ${ }^{\circledR}$ Structures. Dissertation - Delft University of Technology.

65. Whitworth, H.A., Aluko, O., Tomlinson, N. (authors), Gdoutos, E.E. (ed.) (2006). Strength Analysis of Composite Pinned Joints, Fracture of Nano and Engineering Materials and Structures, 1097-1098, Springer, the Netherlands. 
66. Kweon, J.H., Ahn, H.S., Choi, J.H. (2004). A New Method to Determine the Characteristic Lengths of Composite Joints without Testing, Composite Structures, 66(1-4): 305-315.

67. Hamada, H. and Maekawa, ZI. (1996). Strength Prediction of Mechanically Fastened Quasi-Isotropic Carbon/Epoxy Joints. Journal of Composite Materials, 30(14): 1596-1612.

68. Standard Test Method for Bearing Strength of Plastics, Annual Book of ASTM Standards, Item D953-02.

69. Standard Test Method for Pin Type Bearing Test of Metallic Materials, Annual Book of ASTM Standards, Item E238-02.

70. De Jong, T. (1977). Stresses Around Pin-Loaded Holes in Elastically Orthotropic or Isotropic Plates, Journal of Composite Materials, 11: 313-331.

71. Hart-Smith, L.J. (1978). Mechanically-fastened Joints for Advanced Composites - Phenomenological Considerations and Simple Analysis, Douglas Paper 6748: $1-32$.

72. Quinn, W.J. and Matthews, F.L. (1987). The Effect of Stacking Sequence on the Pin-Bearing Strength in GFRP, Journal of Composite Materials, 11: 139-145.

73. Zhang, K.D. and Ueng, C.E.S. (1987). Stresses Around a Pin-Loaded Hole in Orthotropic Plates, Journal of Composite Materials, 18: 432-446.

74. Hyer, M.W. and Klang, E.C. (1985). Contact Stresses in Pin-Loaded Orthotropic Plates, International Journal of Solids and Structures, 21: 957-975. 
75. Hyer, M.W., Klang, E.C., Cooper, D.E. (1987). The Effects of Pin Elasticity, Clearance and Friction on the Stresses in a Pin-Loaded Orthotropic Plate, Journal of Composite Materials, 21: 190-206.

76. Erikson, L.I. (1986). Contact Stresses in Bolted Joints of Composite Laminates, Composite Structures, 6: 57-75.

77. Chen, W.H., Lee, S.S., Yeh, J.T. (1986). Three-dimensional Contact Stress Analysis of a Composite Laminate with Bolted Joint, Composite Structures, 30: 287-297.

78. Smith, P.A., Pascoe, K.J., Polak, C., Stround, D.O. (1986). The Behaviour of Single-Lap Bolted Joints in CFRP Laminates, Composite Structures, 6: 41-45.

79. Camanho, P.P. and Matthews, F.L. (1997). Stress Analysis and Strength Prediction of Mechanically Fastened Joints in FRP: A Review, Composites Part $A$, 28A: $529-547$.

80. Chang, F.K., Scott, R.A., Springer, G.S. (1982). Strength of Mechanically Fastened Composite Joints, Journal of Composite Materials, 16: 470-494.

81. Chang, F.K., Scott, R.A., Springer, G.S. (1984). Failure Strength of Nonlinearly Elastic Composite Laminates Containing a Pin Loaded Hole, Journal of Composite Materials, 18: 465-477.

82. Agarwal, B.L. (1980). Static Strength Prediction of Bolted Joint in Composite Material, American Association of Aeronautics and Astronautics Journal, 8: $1345-1375$. 
83. Whitney, J.M. and Nuismer, R.J. (1974). Stress Fracture Criteria for Laminated Composites Containing Stress Concentrations, Journal of Composite Materials, 8: 253-265.

84. Hashagen, F., Schellekens, J.C.J, de Borst, R. (1995). Finite Element Procedure for Modelling Fibre Metal Laminates, Composite Structures, 32: 255-264.

85. Hashagen, F., de Borst, R., de Vries, T.J. (1999). Delamination Behaviour of Spliced Fibre Metal Laminates. Part 2. Numerical Investigation, Composite Structures, 46: 147-162.

86. de Vries, T.J., Vlot, A., Hashagen, F. (1999). Delamination Behaviour of Spliced Fibre Metal Laminates. Part 1. Experimental Results, Composite Structures, 46: $131-145$.

87. Remmers, J.J.C. and de Borst, R. (2001). Delamination Buckling of Fibre Metal Laminates, Composites Science and Technology, 61: 2207-2213.

88. Linde, P., Pleitner, J., de Boer, H., Carmone, C. (2004). Modeling and Simulation of Fibre Metal Laminates, Proceedings of the 2004 ABAQUS User's Conference, 421-439.

89. Linde, P. and de Boer, H. (2006). Modeling of Inter-Rivet Buckling of Hybrid Composites, Composite Structures, 73: 221-228.

90. Garg, A.C. (1988). Delamination - A Damage Mode in Composite Structures, Engineering Fracture Mechanics, 29(5): 557-584.

91. Reifsnider, K.L., Henneke, E.G., Stinchcomb, W.W., Duke, J.L. (1982). Mechanics of Composite Materials, Recent Advances, Proceedings of the 
International Union of Theoretical Applied Mechanics, Blacksburg, Virginia, Pergamon Press, New York, U.S.A: 339-390.

92. Dharan, C.K.H. (1978). Fracture Mechanics of Composite Materials, Journal of Engineering Materials Technology, 100: 233-247.

93. O’Brien, T.K. (1982). Characterization of Delamination Onset and Growth in a Composite Laminate, Damage in Composite Materials, ASTM STP 775: 140-167.

94. O’Brien, T.K. (1984). Analysis of Local Delaminations and Their Influence on Composite Laminate Behaviour, NASA-TM-85728.

95. Wilkins, D.J., Eisenmann, J.R., Camin, R.A., Margolis, W.S., Benson, R.A. (1982). Characterizing Delamination Growth in Graphite-Epoxy, Damage in Composite Materials, ASTM STP 775: 168-183.

96. Nuismer, R.J. \& Whitney, J.M. (1975). Uniaxial Failure of Composite Laminates Containing Stress Concentrations, ASTM STP 593: 117-142.

97. Wu, E.M. (author), Broutman, L.J. (ed.) (1974). Stress and Fracture of Composites, Composite Materials Vol. V, Academic Press, New York, U.S.A: 191-247.

98. Rybicki, E.F., Schmueser, D.W., Fox, J. (1977). An Energy Release Rate Approach for Stable Crack Growth in the Free Edge Delamination Problem, Journal of Composite Materials, 11: 470-487.

99. Paris, F. (2001). A Study of Failure Criteria of Fibrous Composite Materials, NASA/CR-2001-210661. 
100. Wang J.Z. \& Socie D.F. (1993) Failure Strength and Damage Mechanisms of E-Glass/Epoxy Laminates Under In-Plane Biaxial Compressive Deformation, Journal of Composite Materials, 27(1):40-58.

101. Johnson, W.S. \& Mangalgiri, P.D. (1985). Influence of the Resin on Interlaminar Mixed-Mode Fracture, NASA-TM-87571.

102. Jurf, R.A. \& Pipes, R.B. (1982). Interlaminar Fracture of Composite Materials, Journal of Composite Materials, 16:386-394.

103. Balzani, C. and Wagner, W. (2005). A Simple Model for the Simulation of Delamination in Fibre-Reinforced Composite Laminates Under Mixed-Mode Loading Conditions, Proceedings in Applied Mathematics and Mechanics, 5: $325-$ 326.

104. Schelleckens, J.C.J. and De Borst, R. (1993). On the Numerical Integration of Interface Elements, International Journal for Numerical Methods in Engineering, 36: 43-66.

105. Benzeggagh, M.L. \& Kenane, M. (1996). Measurement of Mixed-Mode Delamination Fracture Toughness of Unidirectional Glass/Epoxy Composites with a Mixed-Mode Bending Apparatus, Composites Science and Technology, 56: 439-449.

106. de Moura, M.F.S.F., Goncalves, J.P.M, Marques, A.T., Castro, P.M.S.T. (1993) Modeling Compression Failure After Low Velocity Impact on Laminated Composites Using Interface Elements, Journal of Composite Materials, 27(15):1462-1479. 
107. Camanho, P.P, Davila, C.G., de Moura, M.F. (2003). Numerical

Simulation of Mixed-Mode Progressive Delamination in Composite Materials, Journal of Composite Materials, 37: 1415-1437.

108. Camanho, P.P., Davila, C.G., Ambur, D.R. (2001). Numerical Simulation of Delamination Growth in Composite Materials, NASA-TP-2001-211041.

109. Camanho, P.P. \& Davila, C.G. (2002). Mixed-Mode Decohesion Finite Elements for the Simulation of Delamination in Composite Materials, NASA-TM2002-211737.

110. Turon, A., Camamho, P.P, Costa, J., Davila, C.G. (2004). An Interface Damage Model for the Simulation of Delamination Under Variable-Mode Ratio in Composite Materials, NASA-TM-2004-213277.

111. June 17, 2008:

www.specialchem4adhesives.com/home/editorial.aspx?id=752.

112. ABAQUS, Inc. (2004). ABAQUS Analysis User's Manual Volume IV:

Elements Version 6.5, 14.1.4-1, USA.

113. Eckold, G. (1994). Design and Manufacture of Composite Structures, Oxford University Press, New York, USA.

114. Maplesoft Inc. (2005). Maple 10 User Manual, 177-230, Canada.

115. Timoshenko, S.P. \& Gere, J.M. (1961). Theory of Elastic Stability $2^{\text {nd }}$

Edition, 348-373, McGraw-Hill Inc., Toronto, ON, Canada.

116. Standard Test Method for Tensile Properties of Polymer Matrix

Composite Materials, Annual Book of ASTM Standards, Item D3039/D3039M-06.

117. Python Programming Language, June 24, 2008: www.python.org. 
118. Mohammadi, S., Owen, D.R.J., Peric, D. (1998). A Combined

Finite/Discrete Element Algorithm for Delamination Analysis of Composites, Finite Elements in Analysis and Design, 28(4): 321-336.

119. Chui, W., Wisnom, M.R., Jones, M. (1992). A Comparison of Failure Criteria to Predict Delamination of Unidirectional Glass/Epoxy Specimens Waisted Through the Thickness, Composites, 23(3): 158-166.

120. Camanho, P.P and Matthews, F.L. (1999). Delamination Onset Prediction In Mechanically Fastened Joints in Composite Laminates, Journal of Composite Materials, 33: 906-927.

121. Davila, C.G. and Johnson E.R. (1993). Analysis of Delamination Initiation in Postbuckled Dropped-Ply Laminates, American Institute of Aeronautics and Astronautics Journal, 31(4): 721-727.

122. Schuecker, C. (2005). Mechanism Based Modeling of Damage and Failure in Fibre Reinforced Polymer Laminates, Dissertation - Vienna University of Technology.

123. Hofslagare, P. (2003). Residual Stress Measurement on Fibre-Metal Laminates, Journal of Neutron Research, 11(4): 215-220. 


\section{APPENDIX A: ORTHOTROPIC PLATE BUCKLING ANALYSIS}

Below is a short Maple program to calculate the critical buckling load for the laterally

unsupported glass prepreg layers modeled as orthotropic plates subject to buckling under fully clamped boundary conditions.

Material Properties Associated with Glass Prepreg...

$>\mathrm{E}[11]:=0.5398 \mathrm{e} 11$ :

$>\mathrm{E}[22]:=0.9412 \mathrm{e} 1:$

$>$ nu[12] $:=.33$ :

$>\mathrm{nu}[21]:=\mathrm{E}[22] * \mathrm{nu}[12] / \mathrm{E}[11]$

$\mathrm{nu}[21]:=0.5753908855 \mathrm{e}-1$

$>\mathrm{G}[12]:=0.27 \mathrm{e} 10$ :

$>\mathrm{t}:=0.125 \mathrm{e}-3$ :

$>\mathrm{t} 2:=0.625 \mathrm{e}-4$ :

Denominator used in matrix calculations...

$>\mathrm{d}:=1-\mathrm{nu}[12]^{*} \mathrm{nu}[21]$

$\mathrm{d}:=.9810121008$

$>\mathrm{Q}:=$ Matrix $(1 . .3,1 . .3,[])$;

Transformation matrix for stress components...

$>\mathrm{T}:=$ Matrix $(1 . .3,1 . .3,[])$ :

$>$ InvT : $=\operatorname{simplify}(1 / T)$

$\operatorname{InvT}:=\operatorname{Matrix}\left(3,3,\left\{(1,1)=\cos (\text { theta })^{\wedge} 2,(1,2)=\sin (\text { theta })^{\wedge} 2,(1,3)=-2 * \sin (\right.\right.$ theta $) * \cos ($ theta $),(2,1)=\sin (\text { theta })^{\wedge} 2,(2,2)=$

$\cos (\text { theta })^{\wedge} 2,(2,3)=2 * \sin (\text { theta })^{*} \cos ($ theta $),(3,1)=\sin$ (theta) ${ }^{*} \cos ($ theta $),(3,2)=-\sin$ (theta) $* \cos ($ theta $\left.),(3,3)=2 * \cos (\text { theta })^{\wedge} 2-1\right\}$, datatype $=$ anything, storage $=$ rectangular, order $=$ Fortran_order, shape $=[]$ )

$>$ Tzero $:=\operatorname{eval}(\mathrm{T},[$ theta $=0])$

Tzero := Matrix $(3,3,\{(1,1)=1,(2,2)=1,(3,3)=1\}$, datatype $=$ anything, storage $=$ rectangular, order $=$ Fortran_order, shape $=[])$ $>$ Tninety $:=\operatorname{eval}(\mathrm{T},[$ theta $=1 / 2 * \mathrm{Pi}])$;

Tninety $:=\operatorname{Matrix}(3,3,\{(1,2)=1,(2,1)=1,(3,3)=-1\}$, datatype $=$ anything, storage $=$ rectangular, order $=$ Fortran_order, shape $=$ [])

$>$ InvTzero := eval(InvT, [theta $=0])$

InvTzero := Matrix $(3,3,\{(1,1)=1,(2,2)=1,(3,3)=1\}$, datatype = anything, storage = rectangular, order $=$ Fortran_order, shape $=$ [])

$>$ InvTninety $:=\operatorname{eval}(\operatorname{InvT},[$ theta $=1 / 2 * \mathrm{Pi}])$;

InvTninety := Matrix $(3,3,\{(1,2)=1,(2,1)=1,(3,3)=-1\}$, datatype $=$ anything, storage $=$ rectangular, order $=$ Fortran_order, shape $=[])$

Defining the relationship between the engineering strain and tensor strain...

$>\mathrm{R}:=$ Matrix $(1 . .3,1 . .3,[])$ :

$>$ InvR : = simplify $(1 / R):$

Defining the Q bar matrix...

$>$ Qbar: $=$

simplify((VectorCalculus[DotProduct])((VectorCalculus[DotProduct])((VectorCalculus[DotProduct])((VectorCalculus[DotPro duct])(InvT, Q), R), T), InvR)): 
Evaluating the above expressions for the three GLARE variants in question...

For Glare 2-2/1-0.4, we have two 0 degree glass prepreg plies, therefore...

$>$ Qbarzero :=

(VectorCalculus[DotProduct])((VectorCalculus[DotProduct])((VectorCalculus[DotProduct])((VectorCalculus[DotProduct])(I nvTzero, Q), R), Tzero), InvR);

Qbarzero := Matrix $(3,3,\{(1,1)=55024805460.0000000,(1,2)=3166077154.00000000,(2,1)=3166077154.00000000,(2,2)=$

$9594173194.00000000,(3,3)=2700000000.00000000\}$, datatype $=$ float $[8]$, storage $=$ rectangular, order $=$ Fortran_order, shape $=[])$

For Glare 3-5/4-0.4 and Glare 4-3/2-0.4 we have ninetydegree plies as well, therefore...

$>$ Qbarninety :=

(VectorCalculus[DotProduct])((VectorCalculus[DotProduct])((VectorCalculus[DotProduct])((VectorCalculus[DotProduct])(I nvTninety, Q), R), Tninety), InvR);

Qbarninety := Matrix $(3,3,\{(1,1)=9594173194.00000000,(2,2)=55024805460.0000000,(1,2)=3166077154.00000000,(2,1)=$

$3166077154.00000000,(3,3)=2700000000.00000000\}$, datatype $=$ float $[8]$, storage $=$ rectangular, order $=$ Fortran $\_$order, shape $\left.=[]\right)$

If we examine the results in (3) and (4) we see that the results differ only in the exchange of two Qbar11 and Qbar22 values...

We can now define the D or bending stiffness matrix for the laminate(s)...

$>$ DGlare2 := 8/3*Qbarzero* $\mathbf{t}^{\wedge} 3$;

DGlare $:=\operatorname{Matrix}(3,3,\{(1,1)=.286587528437500016,(1,2)=0.164899851770833328 \mathrm{e}-1,(2,1)=0.164899851770833328 \mathrm{e}-1,(2$, $2)=0.499696520520833410 \mathrm{e}-1,(3,3)=0.140625000000000004 \mathrm{e}-1\}$, datatype $=$ float $[8]$, storage $=$ rectangular, order $=$

Fortran_order, shape $=[]$ )

$>$ DGlare3 := 1/3*Qbarzero*t^3+1/3*Qbarninety*t^3;

DGlare $3:=$ Matrix $(3,3,\{(1,1)=0.420696475611979232 \mathrm{e}-1,(1,2)=0.412249629427083320 \mathrm{e}-2,(2,1)=0.412249629427083320 \mathrm{e}-$

$2,(2,2)=0.420696475611979232 \mathrm{e}-1,(3,3)=0.351562500000000009 \mathrm{e}-2\}$, datatype $=$ float $[8]$, storage $=$ rectangular, order $=$

Fortran_order, shape $=[]$ )

$>$ DGlare4 := 2*Qbarzero* $\left(1 / 3 *(t+t 2)^{\wedge} 3-1 / 3 * t 2 \wedge 3\right)+2 / 3 * Q^{*}$ barninety* $\left(t 2^{\wedge} 3+0\right)$

DGlare4 := Matrix $(3,3,\{(1,1)=.234413918518779546,(1,2)=0.139134249952747798 \mathrm{e}-1,(2,1)=0.139134249952747798 \mathrm{e}-1,(2$, $2)=0.495562025623857012 \mathrm{e}-1,(3,3)=0.118652343767999980 \mathrm{e}-1\}$, datatype $=$ float $[8]$, storage $=$ rectangular, order $=$

Fortran_order, shape $=[]$ )

In order to calculate the critical buckling load, we need to calculate a few parameters:

Assuming that parameter a is always the length of the plate and the $b$ parameter is the width of the plate:

For Glare 2-2/1-0.4:

$>$ aG2S11 :=0.2814e-2:

$>$ aG2VM $:=0.380 \mathrm{e}-2$ :

$>$ aG2Tresca $:=0.435 \mathrm{e}-2$ :

$>$ bG2S11 :=0.1974e-2:

$>$ bG2VM : $=0.5269 \mathrm{e}-2$ :

$>$ bG2Tresca $:=0.5774 \mathrm{e}-2$ :

For Glare 3-5/4-0.4...

$>$ aG3S11 :=0.315e-2:

$>$ aG3VM $:=0.3886 \mathrm{e}-2$ :

$>$ aG3Tresca $:=0.43615 \mathrm{e}-2$ :

$>$ bG3S11 : $=0.2039 \mathrm{e}-2$ :

$>$ bG3VM $:=0.5577 \mathrm{e}-2$ :

$>$ bG3Tresca $:=0.6078 \mathrm{e}-2$ :

For Glare $4-3 / 2-0.4 \ldots$

$>$ aG4S11 : = 0.3032e-2:

$>$ aG4VM $:=0.3866 \mathrm{e}-2$ :

$>$ aG4Tresca $:=0.4372 \mathrm{e}-2$ :

$>$ bG4S11 :=0.2014e-2:

$>$ bG4VM $:=0.5438 \mathrm{e}-2$ : 


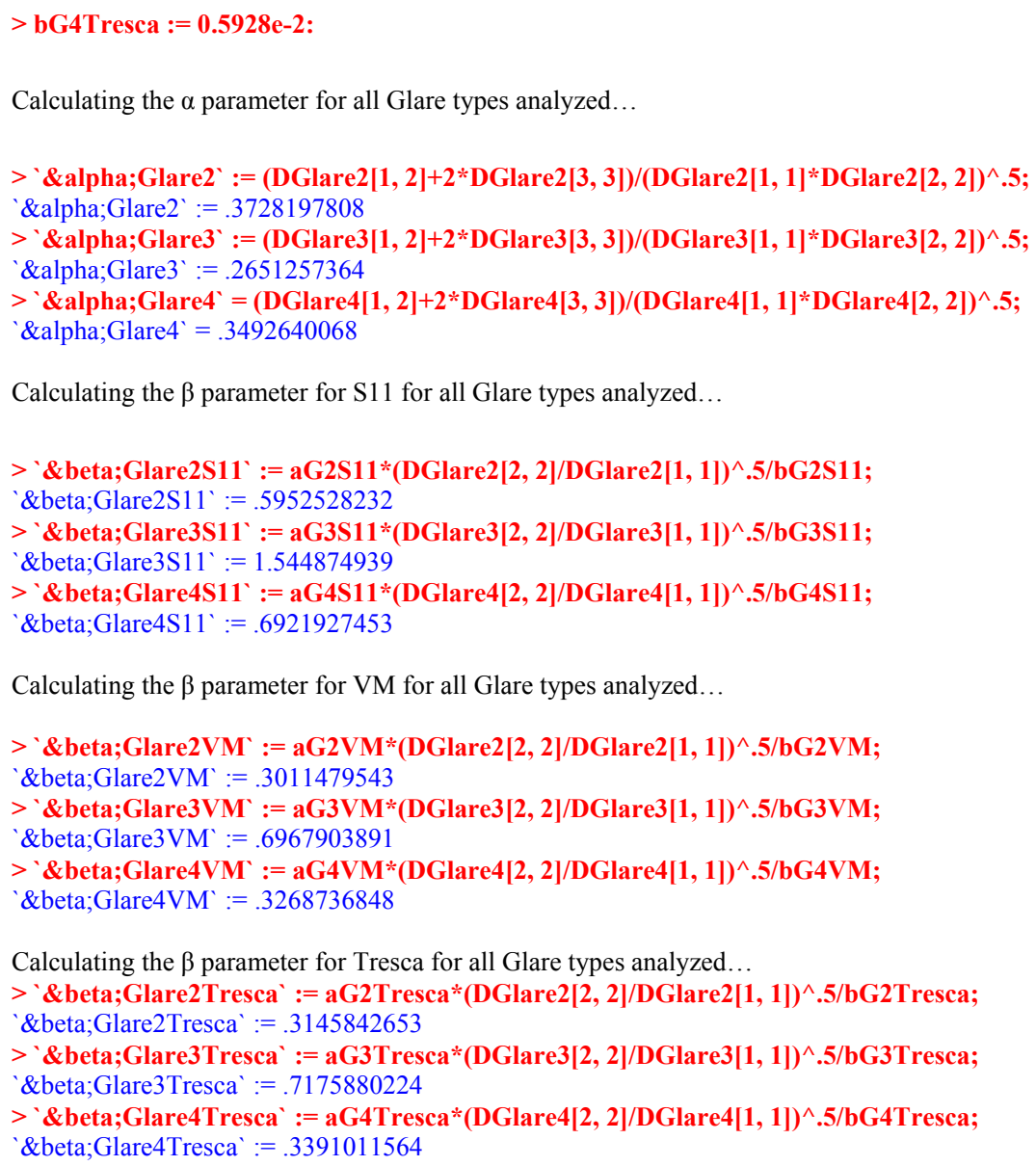

Using the calculated $\alpha \& \beta$ parameters we can now look up the associated $\mathrm{k}$ parameter for plate buckling from the chart provided in Eckold, G. (1994). Design and Manufacture of Composite Structures, 159, Woodhead Publishing Ltd., Cambridge, England. We will use the lines on the chart which correspond to all edges clamped with the loading edge simply supported...

For the $\mathrm{S} 11$ criterion we have...

$>$ kGlare2S11: $:=14:$

$>$ kGlare3S11 $:=6.5$ :

$>$ kGlare4S11 := 11:

For the Von Mises or Octahedral criterion we have...

$>$ kGlare2VM := 15:

$>$ kGlare3VM := 11:

$>$ kGlare4VM := 15:

For the Tresca or Maximum Shear criterion we have...

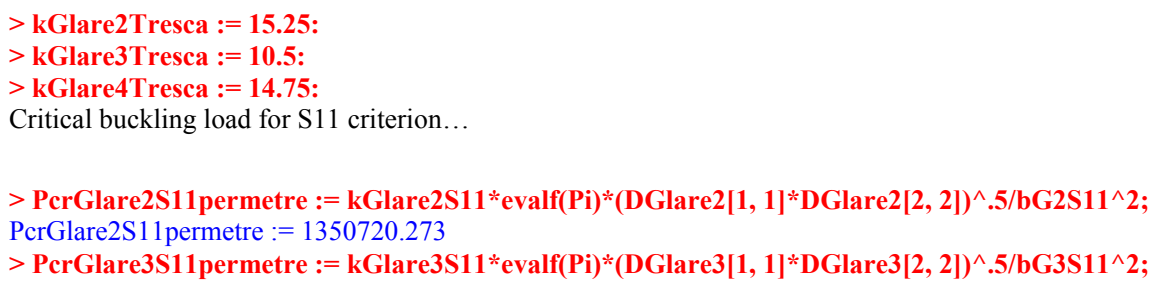


PcrGlare3S11permetre $:=206632.0344$

$>$ PcrGlare4S11 permetre := kGlare4S11*evalf(Pi)*(DGlare4[1, 1]*DGlare4[2, 2] $)^{\wedge} .5 / \mathrm{bG} 4 \mathrm{S11}$ ^2;

PcrGlare4S11permetre :=918257.1789

Critical buckling load for Von Mises criterion...

> PcrGlare2VMpermetre := kGlare2VM*evalf(Pi)*(DGlare2[1, 1]*DGlare2[2, 2])^.5/bG2VM^2;

PcrGlare2VMpermetre $:=203126.4792$

> PcrGlare3VMpermetre := kGlare3VM*evalf(Pi)*(DGlare3[1, 1]*DGlare3[2, 2])^.5/bG3VM^^2;

PcrGlare3VMpermetre $:=46742.30691$

$>$ PcrGlare4VMpermetre := kGlare4VM*evalf(Pi)*(DGlare4[1, 1]*DGlare4[2, 2])^.5/bG4VM²;

P crGlare4VMpermetre :=171752.6443

Critical buckling load for Tresca criterion...

$>$ PcrGlare2Trescapermetre $:=$ kGlare2Tresca*evalf(Pi)*(DGlare2 [1, 1]*DGlare2[2, 2] $)^{\wedge} .5 /$ bG2Tresca^2;

PcrGlare2Trescapermetre $:=171968.1280$

$>$ PcrGlare3Trescapermetre $:=$ kGlare3Tresca*evalf $(P i) *(D G l a r e 3[1,1] * \text { DGlare3 }[2,2])^{\wedge} .5 /$ bG3Tresca^2;

PcrGlare3Trescapermetre $:=37565.28205$

$>$ PcrGlare4Trescapermetre $:=$ kGlare4Tresca*evalf(Pi)*(DGlare4[1, 1]*DGlare4[2, 2] $)^{\wedge} .5 /$ bG4Tresca^2;

PcrGlare4Trescapermetre $:=142123.6034$

Since dimensionally, the above results are given in Newton*metres, we need to multiply the result for each GLARE variant by the thickness of the orthotropic plate being loaded to get our result in Newtons only. The GLARE2-2/1-0.4 specimen had two unidirectional plies per fibre layer; the GLARE3-5/4-0.4 specimen had a single cross ply for each fibre layer and the GLARE4-3/2-0.4 had one zero direction and two 90 degree direction plies per fibre layer. Keeping this in mind, we can now calculate the force applied to each to the fibre layer within each specimen...

Critical buckling load for S11 criterion...

$>$ PcrG2S11 := 2*PcrGlare2S11permetre*t;

PcrG2S11 := 337.6800682

> PcrG3S11 := 2*PcrGlare3S11permetre*t;

PcrG3S11 := 51.65800860

$>$ PcrG4S11 : $=3 *$ PcrGlare4S11 permetre* $\mathrm{t}$;

PcrG4S11 := 344.3464422

Critical buckling load for Von Mises criterion...

$>$ PcrG2VM := 2*PcrGlare2VMpermetre*t;

PcrG2VM $:=50.78161980$

$>$ PcrG3VM := 2*PcrGlare3VMpermetre*t;

PcrG3VM $:=11.68557673$

$>$ PcrG4VM := 3*PcrGlare4VMpermetre* $\mathrm{t}$;

PcrG4VM $:=64.40724162$

Critical buckling load for Tresca criterion...

$>$ PcrG2Tresca $:=2 *$ PcrGlare2Trescapermetre*t;

PcrG2Tresca $:=42.99203200$

> PcrG3Tresca $:=2 *$ PcrGlare3Trescapermetre*t;

PcrG3Tresca $:=9.391320512$

$>$ PcrG4Tresca $:=3 *$ PcrGlare4Trescapermetre*t;

PcrG4Tresca $:=53.29635126$

Now we can calculate the normal stress as a result of the critical buckling load acting on the loaded edge of the orthotropic plate for each GLARE variant. If this normal stress is less than that of the yield stress of the aluminum layers then we have evidence that delamination buckling is indeed the failure mode of GLARE in a pin bearing configuration..

Normal stress required to buckle the laterally unsupported orthotropic plates as defined by the S11 criterion... 


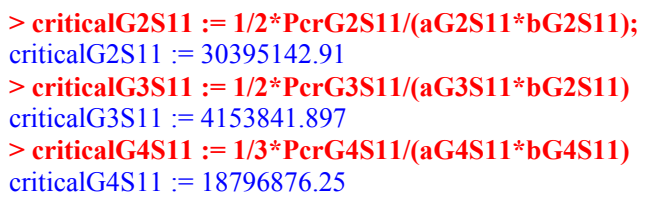

Normal stress required to buckle the laterally unsupported orthotropic plates as defined by the Von Mises criterion...

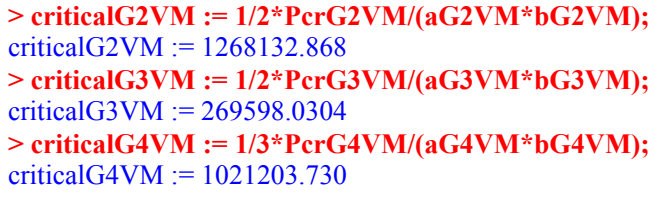

Normal stress required to buckle the laterally unsupported orthotropic plates as defined by the Tresca criterion...

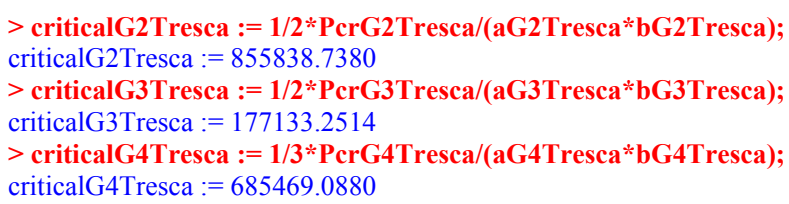

Checking to see if the specimens have all buckled for the Von Mises criterion...

$>$ for $\mathbf{n}$ from 46 to 48 do if $\mathbf{n}<=0.345 \mathrm{e} 9$ then printf(" $\mid \backslash n$ Specimen has buckled for the Von Mises criterion. $\mid \backslash n$ ") end if end do;

Specimen has buckled for the Von Mises criterion.

Specimen has buckled for the Von Mises criterion.

Specimen has buckled for the Von Mises criterion.

Checking to see if the specimens have all buckled for the Tresca criterion..

$>$ for $n$ from 49 to 51 do if $n<=0.345 \mathrm{e} 9$ then $\operatorname{printf("}|| n$ Specimen has buckled for the Tresca criterion. ||$n$ ") end if end do;

Specimen has buckled for the Tresca criterion.

Specimen has buckled for the Tresca criterion.

Specimen has buckled for the Tresca criterion.

All of the examined specimens have buckled. This suggests that the dominant factor in the pin bearing strength of GLARE joints is the yield strength of the aluminum based on the fact that the glass prepreg layers will inevitably buckle once delamination has occurred between the aluminum and the glass (at the yield strength of the aluminum) leaving the glass laterally unsupported...end program... 


\section{APPENDIX B: ISOTROPIC PLATE BUCKLING ANALYSIS}

This short Maple program will develop and implement the equations necessary to calculate the critical load for the buckling of a thin plate according to various prescribed boundary conditions based upon the treatment provided by:

Timoshenko, S.P. \& Gere, J.M. (1961). Theory of Elastic Stability, 348, McGraw-Hill Inc., Toronto, ON, Canada.

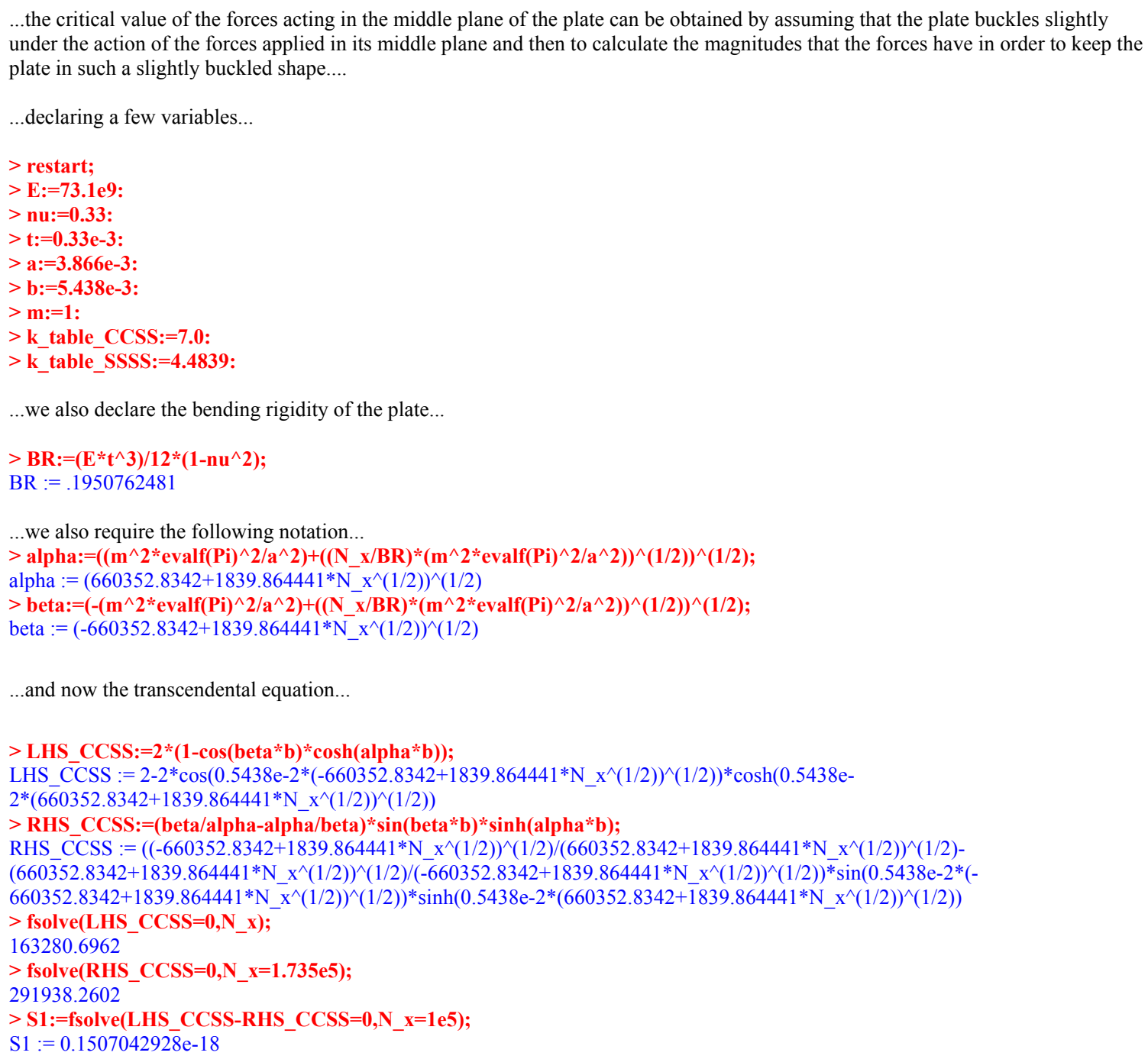




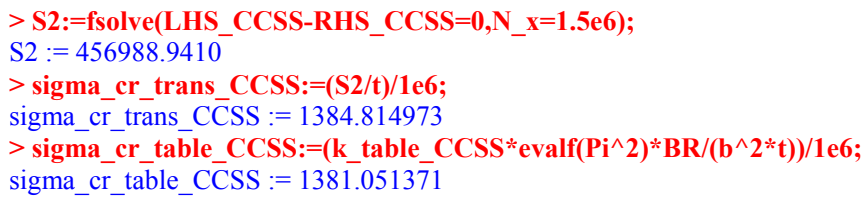

...well we have verified the results produced by the tables in Timoshenko by having arrived at very similar answers by first solving the transcendental equation and then by taking the supplied $\mathrm{k}$ value from the table...these values are too big, so we should consider the simply supported plate next...

$>$ sigma cr table SSSS: $=\left(\mathrm{k}\right.$ table $\mathrm{SSSS}^{*}$ evalf $\left.\left(\mathrm{Pi}^{\wedge} 2\right) * \mathrm{BR} /\left(\mathrm{b}^{\wedge} 2 * \mathrm{t}\right)\right) / 1 \mathrm{e}$;

sigma_cr_table_SSSS :=884.6423206

...this is closer but doesn't quite yet match our boundary conditions since the loaded edge in our examined load case remains free and not laterally unsupported...

...for the case of three edges simply supported or SSS...

...this is simply a subset of a more complex loading scenario whose buckled configuration matches precisely to the situation in question. By virtue of this analogous extension, we must change our aspect ratio from simply $\mathrm{a} / \mathrm{b}$ to the following form...

$>\mathrm{AR}:=2 * \mathrm{~b} / \mathrm{a}$

AR $:=2.813243662$

...and now the corresponding buckling constant from the associated plot...

$>$ k_table_SSS: $=2.3$ :

...which yields the following critical buckling stress...

$>$ sigma_cr_table_SSS: $=\left(\mathrm{k}\right.$ table_SSS*evalf $\left.\left(\mathrm{Pi}^{\wedge} 2\right) * \mathrm{BR} /\left(\mathrm{b}^{\wedge} \mathbf{2}^{*} \mathrm{t}\right)\right) / \mathbf{1} \mathrm{e}$;

sigma_cr_table_SSS $:=453 . \overline{7740218}$

...our calculated yield strength for all GLARE 4 specimens tested is...

> sigma_yield:=365.676:

...and thus our relative error between prediction and measured yield stress is...

$>$ relative_error_sigma_yield_SSS:=((sigma_cr_table_SSS-sigma_yield $) /$ sigma_yield $) * 100$;

relative_error_sigma_yield_SSS $:=24.09182495$

...our average buckling stress for all GLARE 4 specimens tested is...

> sigma_buckle_mean:=420069085.3/1e6:

...producing a relative error of...

$>$ relative error sigma buckle: $=(($ sigma cr table SSS-sigma buckle mean $) /$ sigma buckle mean $) * 100$ relative_error_sigma_buckle $:=8.023665078$

$>$ relative error yield_buckle:=((sigma yield-sigma_buckle_mean)/sigma_buckle_mean $) * 100$; relative_error_yield_buckle $:=-12.94860469$

...These two values are the ones of principal interest and show a relatively small error for the calculation.

...we can also quickly generate the profile which we extracted the buckling constant from by taking a few points, fitting a polynomial through them and calculating the precise $\mathrm{k}$ value corresponding to our aspect ratio... 
...first we'll declare two vectors for the $\mathrm{X}$ and $\mathrm{Y}$ values respectively and a third list of 2D points to plot afterwards...

$>$ phi:=Vector $([0,0.5,0.75,1,1.316,2,2.632,3.0]$, datatype $=$ float $):$

$>\mathrm{k}:=$ Vector($([1.45,1.5,1.75,2.1,2.31,2.4,2.31,2.275]$, datatype=float $):$

$>$ List_phi:=convert(phi,list):

$>$ List_k:=convert(k,list):

$>$ points:=array(1..2,1..nops(List_phi),[List_phi,List_k]):

...now we'll fit a polynomial function through our points, let's try a fourth order first..

> f:=Statistics[PolynomialFit](4,phi,k,x);

$\mathrm{f}:=1.43786535481448307-.673063126567920222 * x+2.33494757224290117 * x^{\wedge} 2$

$1.26958733625679022 * \mathrm{x}^{\wedge} 3+.199273808158586250 * \mathrm{x}^{\wedge} 4$

...let's plot our points and interpolation now...

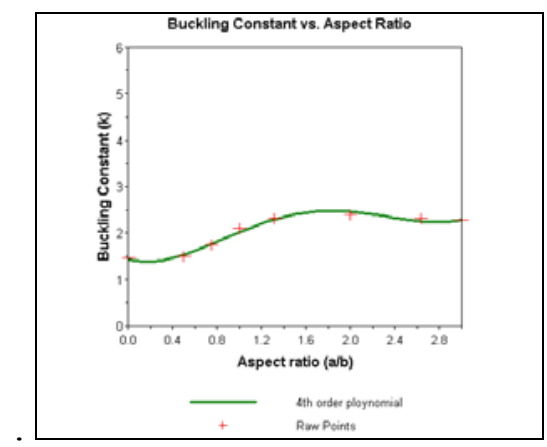

...from the above plot, it is reasonable to state that our polynomial has captured the trend of the data well so now we can evaluate it at our aspect ratio and produce a number for the buckling constant for SSS...

> k_poly:=eval(f,x=AR);

k_poly : $=2.23849283$

...which yields the following critical buckling stress...

$>$ sigma_cr_poly_SSS: $=\left(\mathbf{k} \_\right.$poly*evalf $\left.\left(\mathrm{Pi}^{\wedge} 2\right) * \mathrm{BR} /\left(\mathrm{b}^{\wedge} 2 * \mathrm{t}\right)\right) / \mathbf{1} \mathrm{e} 6$

sigma_cr_poly_SSS :=441.6390845

...producing a relative error for our buckling of...

$>$ relative_error_sigma_buckle_poly:=((sigma_cr_poly_SSS-sigma_buckle_mean)/sigma_buckle_mean)*100; relative_error_sigma_buckle_poly := 5.134869467

...here we see a slight improvement in the calculation by using the polynomial interpolation...

...end program... 


\title{
APPENDIX C: EXTRACTION OF THE ETA PARAMETER IN THE B-K CRITERION
}

\begin{abstract}
...this is a short program to calculate the semi-empirical parameter $\eta$ required in the B-K criterion for modeling delamination in $\mathrm{G} / \mathrm{E}$ laminates...

The procedure involves gathering experimental data from MMB tests in the form of a plot of G_TC (total critical energy) versus G_II/G_T which is the ratio of mode mixity ranging from $100 \%$ Mode I to $100 \%$ Mode II. The polynomial proposed by B-K is a curve fit through these points over the entire range of mode mixes. Camanho et al successfully employed the B-K criterion in their analysis of $\mathrm{C} / \mathrm{E}$ laminates by proposing the minimization of least squares error of the polynomial function proposed by $\mathrm{B}-\mathrm{K}$ in order to solve for the unknown semi-empirical parameter $\eta$.
\end{abstract}

The very same procedure will be applied herein to the original data set provided in the B-K paper on G/E laminates to extract a value of $\eta$ which can be later implemented into ABAQUS to model delamination...

$>\mathrm{G}[\mathrm{IC}]:=118.02$

$\mathrm{G}[\mathrm{IC}]:=118.02$

$>$ G[IIC] := 2905.76;

$\mathrm{G}[\mathrm{IIC}]:=2905.76$

> G[IIC]-G[IC];

2787.74

$>$ G $[1]:=$ G $[$ IC $]:$

$>$ G[2] := 340.35:

$>\mathrm{G}[3]:=568.36$ :

$>\mathrm{G}[4]:=579.62$ :

$>$ G $[5]:=1033.67$ :

$>$ G $[6]:=1821.93$ :

$>$ G[7] := 2457.26:

$>$ G $[8]:=$ G[IIC]:

$>\mathrm{R}[1]:=0.0$ :

$>\mathrm{R}[2]:=.28$ :

$>\mathrm{R}[3]:=.43$ :

$>\mathrm{R}[4]:=.53$ :

$>\mathbf{R}[5]:=.72:$

$>\mathrm{R}[6]:=.82$ :

$>\mathbf{R}[7]:=.91:$

$>\mathbf{R}[8]:=1.00$ :

$>\mathrm{q}:=\operatorname{sum}\left(\left(\mathrm{G}[\mathrm{i}]-\mathrm{G}[\mathrm{IC}]-(\mathrm{G}[\mathrm{IIC}]-\mathrm{G}[\mathrm{IC}])^{*} \mathrm{R}[\mathrm{i}]^{\wedge} \mathrm{eta}\right)^{\wedge} 2, \mathrm{i}=1 . .8\right)$;

$\mathrm{q}:=7771494.308 *(0 . \wedge \text { eta })^{\wedge} 2+\left(222.3300000-2787.740000 * .28^{\wedge} \text { eta }\right)^{\wedge} 2+\left(450.3400000-2787.740000 * .43^{\wedge} \text { eta }\right)^{\wedge} 2+(461.6000000-$

$2787.740000^{*} .53^{\wedge}$ eta $)^{\wedge} 2+\left(915.6500000-2787.740000^{*} .72^{\wedge} \text { eta }\right)^{\wedge} 2+\left(1703.910000-2787.740000 * .82^{\wedge} \text { eta }\right)^{\wedge} 2+(2339.240000-$

$2787.740000 * .91 \wedge$ eta $)^{\wedge} 2+\left(2787.740000-2787.740000 * 1.00^{\wedge} \text { eta }\right)^{\wedge} 2$

$>\mathrm{z}:=\operatorname{diff}(\mathrm{q}$, eta $)$

$\mathrm{z}:=15542988.62 *(0 . \wedge \text { eta })^{\wedge} 2 *(-$ Float(infinity) + Float(undefined)*eta $)+7097.394668 *(222.3300000-$

$2787.740000 * .28^{\wedge}$ eta $) * .28^{\wedge}$ eta $+4705.538248 *\left(450.3400000-2787.740000 * .43^{\wedge}\right.$ eta $) * .43^{\wedge}$ eta $+3539.751110 *(461.6000000-$

$2787.740000^{*} .53^{\wedge}$ eta $) * .53^{\wedge}$ eta $+1831.567855^{*}\left(915.6500000-2787.740000^{*} .72^{\wedge}\right.$ eta $) * .72^{\wedge}$ eta $+1106.459240 *(1703.910000-$

$2787.740000 * .82^{\wedge}$ eta $) * .82^{\wedge}$ eta $+525.8273072 *\left(2339.240000-2787.740000 * .91^{\wedge}\right.$ eta $) * .91^{\wedge}$ eta

$>\operatorname{solve}(z=0$, eta $)$;

2.668647764 


\title{
APPENDIX D: NOMINAL VALUE THRESHOLD EXTRACTION
}

\begin{abstract}
...the following are selected portions (plotting features and functions have not been included) of the post-processing program to extract, manipulate and plot interesting and relevant data as outputted from the progressive damage finite element models performed on GLARE laminates in response to a pin bearing load...
\end{abstract}

we can define the yield strength of 2024-T3 aluminum and our previously calculated modified CCD...

$>$ sigma_y: $=345$ :

$>$ epsilon_y:=2812.838:

$>$ CCD VM:=3.866:

$>$ Delam_Dist_SEM:=4.0275:

...importing the appropriate data...

> SS:=ImportMatrix("C: $\mid \backslash$ Documents and Settings $\backslash \backslash$ Peter $\backslash \backslash$ My Documents $\backslash \backslash$ Theoretical Data $\mid \backslash F E$ Output $\backslash \mid$ Pin Bearing CDM $\backslash$ GLARE $\backslash \backslash$ Residual Stress $\backslash \backslash L R P B \backslash \backslash G 4 \_3 \_2 \backslash \backslash C 3 D 8 I$

Models $\backslash$ G4_3_2_C3D8I_Res_LRPB_Vis005 $\backslash \bar{G} 4$ 3_2_C3D8I_Res_LRPB_Vis005_SS.csv",source $=\mathbf{c s v}$,datatype $=$ anything):

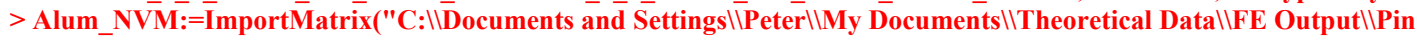
Bearing CDM $\backslash \backslash$ GLARE $\backslash \backslash$ Residual Stress $\backslash \backslash L R P B \backslash \backslash G 4 \quad 3 \quad 2 \| C 3 D 8 I$

Models $\backslash$ G4_3_2_C3D8I_Res_LRPB_Vis005 $\backslash$ G4_3_2_C3D8I_Res_LRPB_Vis005_Alum_NVM.csv",source $=$ csv,datatype $=$ anyt hing):

$>$ Alum_AC:=ImportMatrix("C: $\mid \backslash$ Documents and Settings $\backslash \backslash$ Peter $\| \backslash M y$ Documents $\mid \backslash$ Theoretical Data $\mid \backslash F E$ Output $\mid \backslash P i n$ Bearing CDM $\backslash \bar{G} L A R E \backslash \backslash R$ esidual Stress $\backslash \backslash L R P B \backslash \backslash G 4 \_3 \_2 \backslash \backslash C 3 D 8 I$

Models $\backslash$ G4_3_2_C3D8I_Res_LRPB_Vis005 $\backslash$ G4_3_2_C3D8I_Res_LRPB_Vis005_Alum_AC.csv",source $=$ csv, datatype $=$ anythi ng):

> Alum_PE11:=ImportMatrix("C: $\backslash \backslash$ Documents and Settings $\backslash \backslash$ Peter $\backslash \backslash M y$ Documents $\mid \backslash$ Theoretical Data $\mid \backslash F E$ Output $\backslash \backslash$ Pin Bearing CDM $\backslash \backslash G L A R E \backslash \backslash$ Residual Stress $\backslash \backslash L R P B \backslash \backslash G 4$ 3_2 2 $\backslash$ C3D8I

Models $\backslash$ G4_3_2_C3D8I_Res_LRPB_Vis005 $\backslash \backslash G 4$ 3_2_C3D8I_Res_LRPB_Vis005_Alum_PE11.csv",source $=$ csv,datatype =anyt hing):

> Alum_PEEQ:=ImportMatrix("C: $\backslash \backslash$ Documents and Settings $\backslash \backslash$ Peter $\backslash \backslash M y$ Documents $\backslash \backslash$ Theoretical Data $\backslash \backslash F E$ Output $\backslash \mid P i n$ Bearing CDM $\backslash$ GLARE $\backslash \backslash$ Residual Stress $\backslash L R P B \backslash \backslash G 4 \_3 \_2 \| C 3 D 8 I$

Models $\backslash$ G4_3_2_C3D8I_Res_LRPB_Vis005 $\backslash$ G4_3_2_C3D8I_Res_LRPB_Vis005_Alum_PEEQ.csv", source $=\mathbf{c s v}$,datatype $=$ any thing):

$>$ Coh_NQUADECRT:=ImportMatrix("C: $\backslash \mid$ Documents and Settings $\backslash \backslash$ Peter $\backslash \backslash M y$ Documents $\backslash$ Theoretical Data $\backslash \mid F E$

Output $\mid \backslash$ Pin Bearing CDM $\backslash \backslash$ GLARE $\backslash \backslash$ Residual Stress $\backslash \backslash L R P B \backslash \backslash G 4 \_3 \_2 \backslash C 3 D 8 I$

Models $\backslash$ G4_3_2_C3D8I_Res_LRPB_Vis005 $\backslash$ G4_3_2_C3D8I_Res_LRPB_Vis005_Coh_NQUADECRT.csv",source=csv,dataty pe=anything):

...creating reduced one dimensional arrays of each column from within the imported raw data to facilitate plotting...

...for the aluminum nodal PE11...

$>$ X_Dist_PE11:=[seq(Alum_PE11[i,1],i=1..LinearAlgebra[RowDimension](Alum_PE11))]:

$>$ Avg_Alum_1_PE11:=[seq(abs(Alum_PE11[i,2]/1e-6),i=1..LinearAlgebra[RowDimension](Alum_PE11))]:

$>$ Avg_Alum_2_PE11:=[seq(abs(Alum_PE11[i,3]/1e-6),i=1..LinearAlgebra[RowDimension](Alum_PE11))]:

$>$ Avg_Alum_3_PE11:=[seq(abs(Alum_PE11[i,4]/1e-6),i=1..LinearAlgebra[RowDimension](Alum_PE11))]:

$>$

Avg_Alum_PE11:=[seq(Statistics[Mean]([Avg_Alum_1_PE11[i],Avg_Alum_2_PE11[i],Avg_Alum_3_PE11[i]]),i=1..nops(Avg Alum_1_PE-11))]:

...for the aluminum PEEQ...

$>$ X_Dist_PEEQ:=[seq(Alum_PEEQ[i,1],i=1..LinearAlgebra[RowDimension](Alum_PEEQ))]: 
$>$ Avg_Alum_1_PEEQ:=[seq(abs(Alum_PEEQ[i,2]/1e-6),i=1..LinearAlgebra[RowDimension](Alum_PEEQ))]:

$>$ Avg_Alum_2_PEEQ:=[seq(abs(Alum_PEEQ[i,3]/1e-6),i=1..LinearAlgebra[RowDimension](Alum_PEEQ))]:

$>$ Avg_Alum_3_PEEQ:=[seq(abs(Alum_PEEQ[i,4]/1e-6),i=1..LinearAlgebra[RowDimension](Alum_PEEQ))]:

$>$

Avg_Alum_PEEQ:=[seq(Statistics[Mean]([Avg_Alum_1_PEEQ[i],Avg_Alum_2_PEEQ[i],Avg_Alum_3_PEEQ[i]]),i=1..nops( Avg_Alum_1_PEEQ))]:

...for the aluminum ACYIELD...

$>$ X Dist AC: $=\left[\operatorname{seq}\left(A l u m\right.\right.$ AC $\left.\left.[i, 1], i=1 . . L i n e a r A l g e b r a[R o w D i m e n s i o n]\left(A l u m \_A C\right)\right)\right]:$

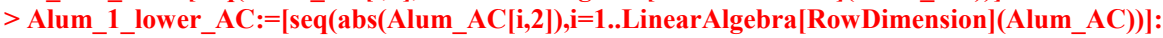

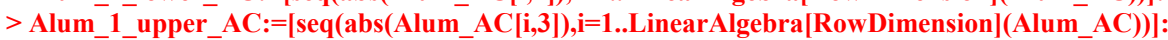

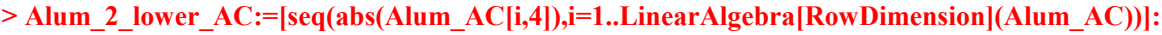

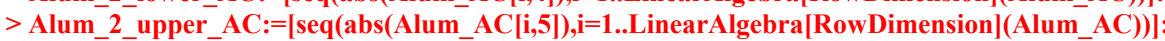

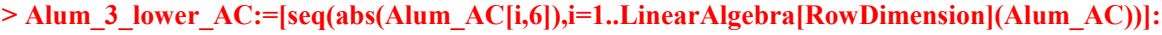

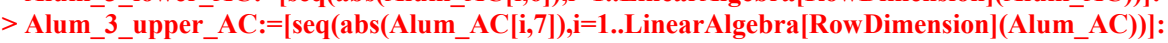

Avg_Alum_AC:=[seq(Statistics[Mean]([Alum_1_lower_AC [i],Alum_1_upper_AC [i],Alum_2_lower_AC [i],Alum_2_upper_AC [i],Alum_3_lower_AC [i],Alum_3_upper_AC [i]]),i=1..nops(Alum_1_lower_AC))]:

...for the cohesive nodal QUADECRT...

$>$ X_Dist_NQUADECRT:=[seq(Coh_NQUADECRT[i,1],i=1..LinearAlgebra[RowDimension](Coh_NQUADECRT))]:

$>$ Coh 1_NQUADECRT:=[seq(Coh_NQUADECRT[i,2],i=1..LinearAlgebra[RowDimension] $($ Coh_NQUADECRT))]:

$>$ Coh_2_NQUADECRT:=[seq(Coh_NQUADECRT[i,3],i=1..LinearAlgebra[RowDimension](Coh_NQUADECRT))]:

$>$ Coh 3_NQUADECRT:=[seq(Coh_NQUADECRT[i,4],i=1..LinearAlgebra[RowDimension](Coh_NQUADECRT))]:

$>$ Coh_4_NQUADECRT:=[seq(Coh_NQUADECRT[i,5],i=1..LinearAlgebra[RowDimension](Coh_NQUADECRT))]:

$>\mathrm{Coh}^{-}$5_NQUADECRT: $=\left[\operatorname{seq}\left(\mathrm{Coh}^{-}\right.\right.$NQUADECRT[i,6],i=1..LinearAlgebra[RowDimension](Coh NQUADECRT))]:

$>$ Coh_6_NQUADECRT:=[seq(Coh_NQUADECRT[i,7],i=1..LinearAlgebra[RowDimension](Coh_NQUADECRT))]:

$>$ Coh_7_NQUADECRT:=[seq(Coh_NQUADECRT[i,8],i=1..LinearAlgebra[RowDimension](Coh_NQUADECRT))]:

$>$ Coh_8_NQUADECRT:=[seq(Coh_NQUADECRT[i,9],i=1..LinearAlgebra[RowDimension](Coh_NQUADECRT))]: $>$

Avg_Coh_NQUADECRT:=[seq(Statistics[Mean]([Coh_1_NQUADECRT[i],Coh_2_NQUADECRT[i],Coh_3_NQUADECRT[i ],Coh_4_NQUADECRT[i],Coh_5_NQUADECRT[i],Coh_6_NQUADECRT[i],Coh_7_NQUADECRT[i],Coh_8_NQUADECRT [i]]),i=1..nops(Coh_1_NQUADECRT))]:

...since our QUADECRT data is somewhat noisy, we can filter it with a moving average algorithm to smooth out local discontinuities and reveal the underlying trend with more clarity...

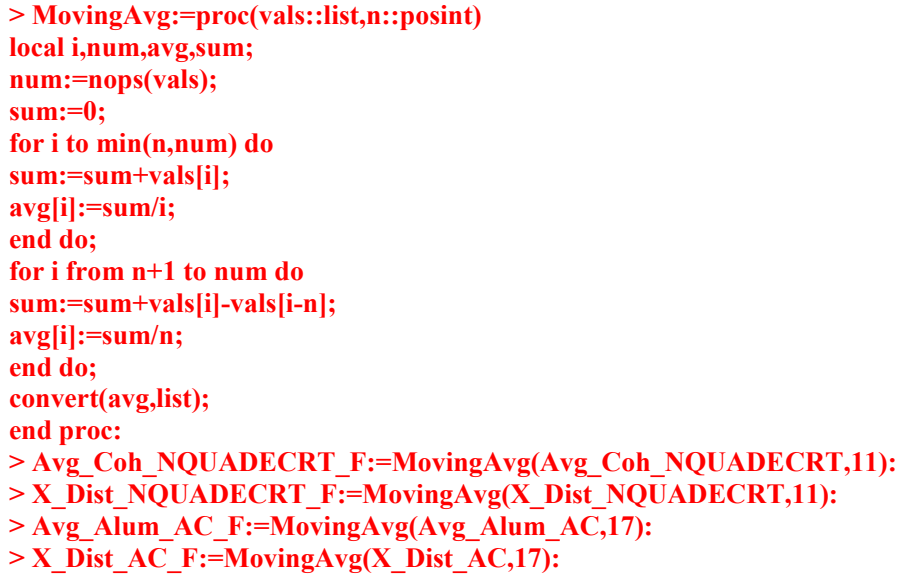

$>$ Avg_Alum_1_PE11_Dist:=array(1..2,1..nops(X_Dist_PE11),[X_Dist_PE11,Avg_Alum_1_PE11]): 
$>$ Avg_Alum_2_PE11_Dist:=array(1..2,1..nops(X_Dist_PE11),[X_Dist_PE11,Avg_Alum_2_PE11]):

$>$ Avg_Alum_3_PE11_Dist:=array(1..2,1..nops(X_Dist_PE11),[X_Dist_PE11,Avg_Alum_3_PE11]):

$>$ Avg_Alum_PE11_Dist:=array(1..2,1..nops(X_Dist_PE11),[X_Dist_PE11,Avg_Alum_PE11]):

...for the alum nodal PEEQ...

$>$ Avg_Alum_1_PEEQ_Dist:=array(1..2,1..nops(X_Dist_PEEQ),[X_Dist_PEEQ,Avg_Alum_1_PEEQ]):

$>$ Avg_Alum_2_PEEQ_Dist:=array(1..2,1..nops(X_Dist_PEEQ),[X_Dist_PEEQ,Avg_Alum_2_PEEQ]):

$>$ Avg_Alum_3_PEEQ_Dist:=array(1..2,1..nops(X_Dist_PEEQ),[X_Dist_PEEQ,Avg_Alum_3_PEEQ]):

$>$ Avg_Alum_PEEQ_Dist:=array(1..2,1..nops(X_Dist_PEEQ),[X_Dist_PEEQ,Avg_Alum_PEEQ]):

...for the alum nodal ACYIELD...

$>$ Alum_1_lower_AC_Dist:=array(1..2,1..nops(X_Dist_AC),[X_Dist_AC,Alum_1_lower_AC]):

$>$ Alum_1_upper_AC_Dist:=array (1..2,1..nops(X_Dist_AC),[X_Dist_AC,Alum_1_upper_AC]):

$>$ Alum 2 lower_AC Dist:=array(1..2,1..nops(X Dist_AC), [X Dist_AC,Alum 2 lower_AC]):

$>$ Alum_2_upper_AC_Dist:=array (1..2,1..nops(X_Dist_AC),[X_Dist_AC,Alum_2_upper_AC]):

$>$ Alum_3_lower_AC_Dist:=array(1..2,1..nops(X_Dist_AC),[X_Dist_AC,Alum_3_lower_AC]):

$>$ Alum_3_upper_AC_Dist:=array(1..2,1..nops(X_Dist_AC),[X_Dist_AC,Alum_3_upper_AC]):

$>$ Avg_Alum_AC_Dist:=array(1..2,1..nops(X_Dist_AC),[X_Dist_AC,Avg_Alum_AC]):

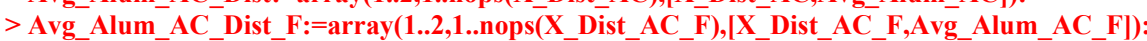

...for the cohesive QUADECRT...

$>$ Coh 1 NQUADECRT Dist:=array(1..2,1..nops(X Dist NQUADECRT),[X Dist NQUADECRT,Coh 1 NQUADECRT]): $>$ Coh_2_NQUADECRT_Dist:=array(1..2,1..nops(X_Dist_NQUADECRT),[X_Dist_NQUADECRT,Coh_2_NQUADECRT]): $>$ Coh_3_NQUADECRT_Dist:=array(1..2,1..nops(X_Dist_NQUADECRT),[X_Dist_NQUADECRT,Coh_3_NQUADECRT]): $>$ Coh_4_NQUADECRT_Dist:=array(1..2,1..nops(X_Dist_NQUADECRT),[X_Dist_NQUADECRT,Coh_4_NQUADECRT]): $>$ Coh_5_NQUADECRT_Dist:=array(1..2,1..nops(X_Dist_NQUADECRT),[X_Dist_NQUADECRT,Coh_5_NQUADECRT]): $>$ Coh_6_NQUADECRT_Dist:=array(1..2,1..nops(X_Dist_NQUADECRT),[X_Dist_NQUADECRT,Coh_6_NQUADECRT]): $>\mathrm{Coh}^{-}$7_NQUADECRT_Dist:=array(1..2,1..nops(X_Dist_NQUADECRT),[X_Dist_NQUADECRT,Coh_7_NQUADECRT]): $>$ Coh_8_NQUADECRT_Dist:=array(1..2,1..nops(X_Dist_NQUADECRT),[X_Dist_NQUADECRT,Coh_8_NQUADECRT]): $>$

Avg_Coh_NQUADECRT_Dist:=array(1..2,1..nops(X_Dist_NQUADECRT),[X_Dist_NQUADECRT,Avg_Coh_NQUADECRT l):

Avg_Coh_NQUADECRT_Dist_F:=array(1..2,1..nops(X_Dist_NQUADECRT_F),[X_Dist_NQUADECRT_F,Avg_Coh_NQUA DECRT_- F]):

...next we'll look and see if our plasticity measures and active yield flags decay near our CCD as predicted by the YDB mechanism...

...for the alum PE11...

...we'll call 50 microstrain as the end of plasticity since that borders on the minimal signal due to the inherent noise of analog electronics, 100 microstrain can be used as well since they are both very small numbers. In general, 100 microstrain was used...

> Alum_1_PE11_end:=nearest(Avg_Alum_1_PE11,X_Dist_PE11,50);

Alum_1_PE11_end := [[3.58827, 7.63], [3.58827, 7.63], [3.58833, 7.65], [3.58834, 7.65], [4.08905, 0.275e-2]]

$>$ Alum 2 PE11 end:=nearest(Avg_Alum 2 PE11,X Dist PE11,50);

Alum_2_PE11_end :=[[3.58813, 1.44] $,[3.588 \overline{14}, 1.44],[3.58 \overline{8} 27,1.48],[3.58827,1.48],[3.58833,1.48]]$

$>$ Alum 3 PE11 end:=nearest(Avg_Alum 3 PE11,X Dist PE11,50);

Alum_3_PE11_end := [[3.58827, 7.57], [3.58827, 7.57], [3.58833, 7.59], [3.58834, 7.59], [4.08905, 0.297e-2]]

> Avg_Alum_PE11_end:=nearest(Avg_Alum_PE11,X_Dist_PE11,50);

Avg_Alum_PE11_end :=[[3.58827, 5.560000000], [3.58827, 5.560000000], [3.58833, 5.573333333], [3.58834, 5.5733333333], [4.08905, 0.1993000000e-2]]

...for the alum PEEQ...

>Alum_1_PEEQ_end:=nearest(Avg_Alum_1_PEEQ,X_Dist_PEEQ,50);

Alum 1 $\mathrm{PEEQ}$ end $:=[[3.58827,8.06],[3.58827,8.06],[3.588 \overline{3} 3,8.08],[3.58834,8.08],[4.08905,0.290 \mathrm{e}-2]]$

$>$ Alum_2_PEEQ_end:=nearest(Avg_Alum_2_PEEQ,X_Dist_PEEQ,50);

Alum_2_PEEQ_end := [[3.58813, 1.49], [3.58814, 1.50], [3.58827, 1.54], [3.58827, 1.54], [3.58833, 1.54]] 
> Alum_3_PEEQ_end:=nearest(Avg_Alum_3_PEEQ,X_Dist_PEEQ,50);

Alum_3_PEEQ_end :=[[3.58827, 7.99], [3.58833, 8.01], [3.58834, 8.02], [4.08905, 0.313e-2], [4.08905, 0.300e-2]]

$>$ Avg_Alum_PEEQ_end:=nearest(Avg_Alum_PEEQ,X_Dist_PEEQ,50);

Avg_Alum_PEEQ_end $:=[[3.58827,5.8633333333],[3.58833,5.876666667],[3.58834,5.880000000],[4.08905,0.2099666667 \mathrm{e}-2]$, [4.08905, 0.1984000000e-2]]

...for the alum AC YIELD, we'll call $2.5 \%$ as the minimum...

$>$ Alum_1_lower_AC_end:=nearest(Alum_1_lower_AC,X_Dist_AC,0.1);

Alum_1_lower_AC_end := [[3.11123, 1.09151], [3.11124, 1.09147], [3.58813,.158], [3.58814, .158], [3.58827, .158]]

$>$ Alum_1_upper_AC_end:=nearest(Alum_1_upper_AC,X_Dist_AC,0.1);

Alum_1_upper_AC_end $:=[[3.58833, .274],[3.58834, .274],[4.08905,0],[4.08905,0],[4.08913,0]]$

$>$ Alum_2_lower_AC_end:=nearest(Alum_2_lower_AC,X_Dist_AC,0.1);

Alum_2_lower_AC_end :=[[3.58833, .250], [3.58834, .250], [4.08905, 0.431e-4], [4.08905, 0.388e-4], [4.08913, 0]]

$>$ Alum_2_upper_AC_end:=nearest(Alum_2_upper_AC,X_Dist_AC,0.1);

Alum_2_upper_AC_end :=[[3.58833,.250], [3.58834,.250], [4.08905, 0.443e-4], [4.08905, 0.400e-4], [4.08913, 0.119e-5]]

$>$ Alum_3 lower_AC_end:=nearest(Alum_3_lower_AC,X_Dist_AC,0.1);

Alum_3_lower_AC_end :=[[3.58833, .274], [3.58834, .274], [4.08905, 0.471e-5], [4.08905, 0], [4.08913,0]]

$>$ Alum_3_upper_AC_end:=nearest(Alum_3_upper_AC,X_Dist_AC,0.1);

Alum_3_upper_AC_end :=[[3.11123, 1.0915], $[3.11124,1.09151],[3.58813, .158],[3.58814, .158],[3.58827, .158]]$

$>$ Avg_Alum_AC end:=nearest(Avg_Alum_AC,X Dist_AC,0.1);

Avg_Alum_AC_end := [[3.58833, $.22 \overline{7} 33333 \overline{3} 3],[3 . \overline{5} 8834 \overline{4}, .2273333333],[4.08905,0.3015166667 \mathrm{e}-4],[4.08905,0.2700000000 \mathrm{e}-4]$, [4.08913, 0.5881666667e-5]]

...for the cohesive interface QUADECRT, we'll use $2.5 \%$ as the minimum...

>Coh_1_NQUADECRT_end:=nearest(Coh_1_NQUADECRT,X_Dist_NQUADECRT,0.1);

Coh $1-\overline{N Q U A D E C R T}$ end $:=[[3.1114, .191],[3.1114, .191],[3.58813,0.781 \mathrm{e}-1],[3.58814,0.781 \mathrm{e}-1],[3.58827,0.781 \mathrm{e}-1]]$

$>$ Coh_2_NQUADECRT_end:=nearest(Coh_2_NQUADECRT,X_Dist_NQUADECRT,0.1);

Coh_2_NQUADECRT_end := [[3.58827,.109], [3.58827, .109], [3.58836, .108], [3.58836,.108], [3.5884, .108]]

$>$ Coh_3_NQUADECRT_end:=nearest(Coh_3_NQUADECRT,X_Dist_NQUADECRT,0.1);

Coh_3_NQUADECRT_end := [[2.71737,.158], [2.71739,.158], [3.11093,.103], [3.11094,.103], [3.11113,.103]]

$>$ Coh_4_NQUADECRT_end:=nearest(Coh_4_NQUADECRT,X_Dist_NQUADECRT,0.1);

Coh_4_NQUADECRT_end := [[2.34231,.243], [2.34231,.243], [2.71667,.134], [2.71668,.134], [2.71696,.134]]

$>$ Coh 5 NQUADECRT end:=nearest(Coh 5 NQUADECRT,X Dist_NQUADECRT,0.1);

Coh_5_NQUADECRT_end : $=[[2.71696, .134],[2.71696, .134],[2.71728, .133],[2.7173, .133],[2.71737, .133]]$

$>$ Coh_6_NQUADECRT_end:=nearest(Coh_6_NQUADECRT,X_Dist_NQUADECRT,0.1);

Coh_6_NQUADECRT_end $:=[[2.71737, .157],[2.71739, .157],[3.11093, .103],[3.11094, .103],[3.11113, .103]]$

$>$ Coh 7 NQUADECRT end:=nearest(Coh 7 NQUADECRT,X Dist NQUADECRT,0.1);

Coh_7_NQUADECRT_end :=[[3.58827, .109], [3.58827, .109], [3.58836,.108], [3.58836, .108], [3.5884, .108]]

$>$ Coh 8 NQUADECRT end:=nearest(Coh 8 NQUADECRT,X Dist NQUADECRT,0.1);

Coh_8_NQUADECRT_end :=[[3.1114, .191], [3.1114, .191], [3.58813, 0.781e-1], [3.58814, 0.781e-1], [3.58827, 0.781e-1]]

$>$ Avg_Coh_NQUADECRT_end:=nearest(Avg_Coh_NQUADECRT,X_Dist_NQUADECRT,0.025);

Avg_Coh_NQUADECRT_end $:=[[3.5884,0.5962500000 \mathrm{e}-1],[3.58841,0.5962500000 \mathrm{e}-1],[4.08905,0.2444500000 \mathrm{e}-1],[4.08905$,

$0.2444500000 \mathrm{e}-1]$, [4.08913, $0.2444500000 \mathrm{e}-1]]$

...using a least squares interpolation over the region near our point of interest, namely the nominal value...

...for the alum PE11...

>f Alum 1 PE11 end:=CurveFitting[LeastSquares](Alum 1 PE11_end,x);

f_Alum_1_PE11_end := 62.36768351-15.2516919047870499*x

$>$ f Alum 2 PE11 end:=CurveFitting[LeastSquares](Alum 2 PE11 end,x);

f_Alum_2_PE11_end :=-852.0019949+237.851662403995874*x

$>$ f Alum 3 PE11 end:=CurveFitting[LeastSquares](Alum 3 PE11 end, $x$ );

f Álum 3_PE11 end : = 61.87615350-15.1314316963653646*x

$>\mathbf{f}$ Avg_Alum_PE11_end:=CurveFitting[LeastSquares](Avg_Alum_PE11_end,x);

f_Avg_Alum_PE11_end := 45.44250032-11.1127292387434977*x

...for the alum PEEQ...

>f_Alum_1_PEEQ_end:=CurveFitting[LeastSquares](Alum_1_PEEQ_end,x);

f_Alum_1_PEEQ_end $:=65.87794211-16.1101085535758415 * x$ 
$>$ f Alum 2 PEEQ_end:=CurveFitting[LeastSquares](Alum_2_PEEQ_end,x);

f_Alum_2_PEEQ_end $:=-964.3629028+269.181585677639474 *{ }^{*} \mathrm{x}$

$>$ f Alum 3 PEEQ end:=CurveFitting[LeastSquares](Alum 3 PEEQ end,x);

f_Ālum_3_PEEQ_end $:=65.36101088-15.9836501640303120 * \bar{x}$

$>$ f_Avg_Alum_PEEQ_end:=CurveFitting[LeastSquares](Avg_Alum_PEEQ_end,x);

f_Avg_Alum_PEEQ_end $:=47.94740252-11.7253053859010912{ }^{*} \mathrm{x}$

...for the alum AC Yield...

$>$ f_Alum 1 lower_AC end:=CurveFitting[LeastSquares](Alum 1 lower_AC end,x); f_Alum_1_lower_AC_end $:=7.180885253-1.95722768549361992 * \bar{x}$

$>$ f_Alum_1_upper_AC_end:=CurveFitting[LeastSquares](Alum_1_upper_AC_end,x); f_Alum_1_upper_AC_end $:=2.237495032-.547188329449544098 * x$

$>$ f_Alum_2_lower_AC_end:=CurveFitting[LeastSquares](Alum_2_lower_AC_end,x); f_Alum_2_lower_AC_end $:=2.041314461-.499204912809516454 * x$

$>$ f_Alum_2_upper_AC_end:=CurveFitting[LeastSquares](Alum_2_upper_AC_end,x); f Alum 2 upper $\mathrm{AC}$ end $:=2.041305885-.499202523022842958^{*} \mathrm{x}$

$>$ f_Alum_3_lower_AC_end:=CurveFitting[LeastSquares](Alum_3_lower_AC_end,x); f_Alum_3_lower_AC_end $:=2.237483783-.547185194517784690 * \bar{x}$

$>$ f_Alum_3_upper_ĀC_end:=CurveFitting[LeastSquares](Alum_3_upper_AC_end,x);

f_Alum_3_upper_AC_end $:=7.180998099-1.95725913474357350 * x$

$>$ f_Avg_Alum_AC_end:=CurveFitting[LeastSquares](Avg_Alum_AC_end,x);

f_Avg_Alum_AC_end $:=1.856262602-.453951280817590564 * x$

...for the cohesive interface QUADECRT...

> f_Coh_1_NQUADECRT_end:=CurveFitting[LeastSquares](Coh_1_NQUADECRT_end,x); f Coh $1-$ NQUADECRT end $:=.9277696691-.236796834914717092 * \mathrm{x}$

>f_Coh_2_NQUADECRT_end:=CurveFitting[LeastSquares](Coh_2_NQUADECRT_end,x); f_Coh_2_NQUADECRT_end $:=32.16554467-8.93371757926919763{ }^{*} \mathrm{x}$

>ffCoh_3_NQUADECRT_end:=CurveFitting[LeastSquares](Coh_3_NQUADECRT_end,x); f $\bar{C}$ oh $3-\overline{N Q U A D E C R T}$ end $:=.5376958428-.139728653084233406^{*} \mathrm{x}$

$>$ f_Coh_4_NQUADECRT_end:=CurveFitting[LeastSquares](Coh_4_NQUADECRT_end,x); f_Coh_4_NQUADECRT_end $:=.9248130104-.291085736499976822{ }^{\bar{*}} \mathrm{x}$

$>$ f Coh 5 NQUADECRT end:=CurveFitting[LeastSquares](Coh 5 NQUADECRT end,x); f Coh 5 NQUADECRT end $:=7.535070519-2.72403258655893676^{*} \mathrm{x}$

$>$ f Coh 6 NQUADECRT end:=CurveFitting[LeastSquares](Coh 6 NQUADECRT end,x); f_Coh_6_NQUADECRT_end $:=.5297922820-.137188132119065470{ }^{*} \mathrm{x}$

$>$ f Coh 7 NQUADECRT end:=CurveFitting[LeastSquares](Coh 7 NQUADECRT end,x); f_Coh_7_-_QUADECRT_end $:=32.16554467-8.93371757926919763{ }^{*} \mathrm{x}$

$>$ f Coh 8 NQUADECRT end:=CurveFitting[LeastSquares](Coh 8 NQUADECRT end,x); f_Coh_8_NQUADECRT_end $:=.9277696691-.236796834914717092 \bar{*}^{\mathrm{x}}$

$>$ f Avg Coh NQUADECRT end:=CurveFitting[LeastSquares](Avg Coh NQUADECRT end,x);

f_Avg_Coh_NQUADECRT_end $:=.3117664618-0.702656088557493952 \mathrm{e}-1 * \overline{\mathrm{x}}$

...solving the polynomial interpolation for the distance from the pin hole corresponding to the nominal value...

...for the alum PE11...

> Dist_Alum_1_PE11_end:=fsolve(f_Alum_1_PE11_end=1,x);

Dist Alum 1 PE11 end $:=4.023663992$

$>$ Dist_Alum_2_PE11_end:=fsolve(f_Alum_2_PE11_end=1,x);

Dist Alum $2-\overline{P E} 11$ end $:=3.586277 \overline{2} 04$

$>$ Dist_Alum_3_PE11_end:=fsolve(f_Alum_3_PE11_end=1,x);

Dist Alum 3 PE11 end :=4.023158859

$>$ Dist_Avg_Alum_PE11_end:=fsolve(f_Avg_Alum_PE11_end=1,x);

Dist_Avg_Alum_PE11_end := 3.999242613

...for the alum PEEQ...

> Dist Alum 1 PEEQ end:=fsolve(f Alum 1 PEEQ end=1,x);

Dist_Alum_1_PEEQ_end $:=4.027157353$

> Dist_Alum_2_PEEQ_end:=fsolve(f_Alum_2_PEEQ_end=1,x); 
Dist Alum 2 PEEQ end $:=3.586288788$

> Dist_Alum_3_PEEQ_end:=fsolve(f_Alum_3_PEEQ_end=1,x);

Dist Alum 3 PEEQ end $:=4.026677901$

$>$ Dist_Avg_Alum_PEEQ_end:=fsolve(f_Avg_Alum_PEEQ_end=1,x);

Dist_Avg_Alum_PEEQ_end $:=4.003938572$

...for the alum AC YIELD, please note that since this is an elemental status variable, the length it refers to is the end point of the last element which has integration points that are actively yielding and thus its values will be slightly higher. As a side, it shows that in fact the plastic strains have dropped to nominal levels within that element...

> Dist_Alum_1_lower_AC_end:=fsolve(f_Alum_1_lower_AC_end $=0.05, \mathbf{x})$;

Dist Alum 1 lower AC end : $=3.643360099$

$>$ Dist_Alum_1_upper_AC_end:=fsolve(f_Alum_1_upper_AC_end $=0.05, x)$;

Dist Alum 1 upper A $\bar{C}$ end $:=3.997700452$

$>$ Dist_Alum_2_lower_AC_end:=fsolve(f_Alum_2_lower_AC_end $=0.05, x)$;

Dist_Alum_2_lower_AC_end : $=3.988972083$

$>$ Dist_Alum_2_upper_AC_end:=fsolve(f_Alum_2_upper_AC_end $=0.05, x)$;

Dist_Alum_2_upper_AC $\bar{C}$ end $:=3.988974000$

$>$ Dist_Alum_3 lower_AC_end:=fsolve(f_Alum_3 lower_AC_end $=0.05, x)$;

Dist_Âlum_3_lower_AC_end : $=3.997702798$

$>$ Dist_Alum_3_upper_AC_end:=fsolve(f_Alum_3_upper_AC_end $=0.05, x)$;

Dist_Alum_3_upper_AC $\bar{C} \_$end $:=3.643359212$

$>$ Dist_Avg_Alum_AC_end:=fsolve(f_Avg_Alum_AC_end $=0.05, x)$;

Dist_Avg_Alum_AC_end : $=3.978978975$

...for the cohesive interface QUADECRT...

> Dist Coh 1_NQUADECRT end:=fsolve(f_Coh_1_NQUADECRT_end $=0.025, \mathrm{x})$;

Dist_Coh_1_NQUADECRT_end := 3.812422871

$>$ Dist Coh 2 NQUADECRT end:=fsolve(f Coh 2 NQUADECRT end $=0.025, x)$;

Dist_Coh_2_NQUADECRT_end $:=3.597667420$

> Dist Coh 3 NQUADECRT end:=fsolve(f Coh 3 NQUADECRT end $=0.025, x)$;

Dist_Coh_3_NQUADECRT_end $:=3.669224826$

$>$ Dist Coh 4 NQUADECRT end:=fsolve(f Coh 4 NQUADECRT end $=0.025, x)$;

Dist_Coh_4_NQUADECRT_end :=3.091230169

$>$ Dist_Coh 5 NQUADECRT end:=fsolve(f_Coh 5 _NQUADECRT end $=0.025, x)$;

Dist_Coh_5_NQUADECRT_end $:=2.756967943$

> Dist_Coh_6_NQUADECRT_end:=fsolve(f_Coh_6_NQUADECRT_end $=0.025, \mathrm{x})$;

Dist_Coh_6_NQUADECRT_end :=3.679562323

> Dist_Coh_7_NQUADECRT_end:=fsolve $(\mathrm{f}$-Coh_8_NQUADECRT_end $=\mathbf{0 . 0 2 5}, \mathrm{x})$;

Dist_Coh_7_NQUADECRT_end := 3.812422871

> Dist_Coh_8_NQUADECRT_end:=fsolve(f_Coh_8_NQUADECRT_end $=0.025, \mathrm{x})$;

Dist Coh 8 NQUADECRT end $:=3.812422871$

$>$ Dist_Avg_Coh_NQUADECRT_end:=fsolve(f_Avg_Coh_NQUADECRT_end=0.025,x);

Dist_Avg_Coh_NQUADECRT_end $:=4.081178068$

...we can quickly calculate the relative difference between the progressive damage results and the CCD/experimental ones...

...for the alum PE11 end distance compared to VM CCD...

>CCD_Rel_Diff_Alum_1_PE11_end:=RelDiff(Dist_Alum_1_PE11_end,CCD_VM);

CCD_Rel_Diff_Alum_1_PE11_end $:=4.078220176$

$>$ CCD_Rel_Diff_Alum_2_PE11_end:=RelDiff(Dist_Alum_2_PE11_end,CCD_VM);

CCD_Rel_Diff_Alum_2_PE11_end : = -7.235457734

$>$ CCD_Rel_Diff_Alum_3_PE11_end:=RelDiff(Dist_Alum_3_PE11_end,CCD_VM);

CCD_Rel_Diff_Alum_3_PE11_end $:=4.065154139$

$>$ CCD_Rel_Diff_Avg_Alum_PE11_end:=RelDiff(Dist_Avg_Alum_PE11_end,CCD_VM);

CCD_Rel_Diff_Avg_Alum_PE11_end :=3.446523875

...for the alum PEEQ end distance compared to VM CCD...

$>$ CCD_Rel_Diff_Alum_1_PEEQ_end:=RelDiff(Dist_Alum_1_PEEQ_end,CCD_VM);

CCD Rel Diff Alum 1_PEEQ end $:=4.168581298$ 
>CCD_Rel_Diff_Alum_2_PEEQ_end:=RelDiff(Dist_Alum_2_PEEQ_end,CCD_VM);

CCD_Rel_Diff_Alum_2_PEEQ_end $:=-7.235158096$

$>$ CCD Rel Diff_Alum 3 PEEQ end:=RelDiff(Dist_Alum 3 PEEQ end,CCD_VM);

CCD_Rel_Diff_Alum_3_PEEQ_end $:=4.156179540$

$>$ CCD Rel Diff Avg Alum PEEQ end:=RelDiff(Dist_Avg_Alum PEEQ end,CCD VM);

CCD_Rel_Diff_Avg_Alum_PËEQ_end := 3.567992033

...for the alum AC YIELD end distance compared to VM CCD...

$>$ CCD_Rel_Diff Alum 1 lower_AC end:=RelDiff(Dist_Alum 1 lower AC end,CCD_VM); CCD_Rel_Diff_Alum_1_lower_AC_end $:=-5.758921392$

$>$ CCD_Rel_Diff_Alum_1_upper_AC_end:=RelDiff(Dist_Alum_1_upper_AC_end,CCD_VM); CCD_Rel_Diff_Alum_1_upper_AC _end $:=3.406633523$

$>$ CCD_Rel_Diff_Alum_2_lower_AC_end:=RelDiff(Dist_Alum_2_lower_AC_end,CCD_VM); CCD_Rel_Diff_Alum_2_lower_AC_end :=3.180860916

$>$ CCD_Rel_Diff_Alum_2_upper_AC_end:=RelDiff(Dist_Alum_2_upper_AC_end,CCD_VM); CCD Rel Diff Alum 2 upper AC end $:=3.180910502$

$>$ CCD_Rel_Diff_Alum_3_lower_AC_end:=RelDiff(Dist_Alum_3_lower_AC_end,CCD_VM); CCD_Rel_Diff_Alum_3_lower_AC_end $:=3.406694206$

$>$ CCD_Rel_Diff_Alum_3_upper_AC_end:=RelDiff(Dist_Alum_3_upper_AC_end,CCD_VM); CCD Rel Diff Alum 3 upper A $\bar{C}$ end $:=-5.758944335$

$>$ CCD_Rel_Diff_Avg_Alum_AC_end:=RelDiff(Dist_Avg_Alum_AC_end,CCD_VM);

CCD Rel_Diff_Avg_Alum AC end $:=2.922373901$

...for the cohesive interface QUADECRT end distance compared to the value taken from the SEM micrographs...

> Delam_SEM_Rel_Diff_Coh_1_NQUADECRT_end:=RelDiff(Dist_Coh_1_NQUADECRT_end,Delam_Dist_SEM); Delam SEM Rel Diff Coh 1 NQ̈UADECRT end $:=-5.340214252$

$>$ Delam_SEM_Rel_Diff_Coh_2_NQUADECRT_end:=RelDiff(Dist_Coh_2_NQUADECRT_end,Delam_Dist_SEM); Delam SEM Rel Diff Coh 2 NQUADECRT end $:=-10.67244146$

$>$ Delam_SEM_Rel_Diff_Coh_3_NQUADECRT_end:=RelDiff(Dist_Coh_3_NQUADECRT_end,Delam_Dist_SEM); Delam SEM Rel Diff Coh 3 NQUADECRT end $:=-8.895721266$

$>$ Delam_SEM_Rel_Diff_Coh 4 NQUADECRT end:=RelDiff(Dist_Coh 4 NQUADECRT end,Delam_Dist_SEM);

Delam_SEM_Rel_Diff_Coh_4_NQUADECRT_end := -23.24692318

$>$ Delam SEM Rel Diff Coh 5 NQUADECRT end:=RelDiff(Dist_Coh 5 NQUADECRT end,Delam_Dist_SEM);

Delam_SEM_Rel_Diff_Coh_5_NQUADECRT_end $:=-31.54641979$

$>$ Delam SEM Rel Diff Coh 6 NQUADECRT end:=RelDiff(Dist_Coh 6 NQUADECRT end,Delam Dist_SEM);

Delam_SEM_Rel_Diff_Coh_6_NQUADECRT_end $:=-8.639048467$

$>$ Delam SEM Rel Diff Coh 7 NQUADECRT end:=RelDiff(Dist_Coh 7 NQUADECRT end,Delam Dist SEM);

Delam_SEM_Rel_Diff_Coh_7_NQUUADECRT_end $:=-5.340214252$

$>$ Delam SEM Rel Diff Coh 8 NQUADECRT end:=RelDiff(Dist Coh 8 NQUADECRT end,Delam Dist_SEM);

Delam_SEM_Rel_Diff_Coh_8_NQUADECRT_end $:=-5.340214252$

$>$ Delam SEM Rel Diff_Avg_Coh NQUADECRT_end:=RelDiff(Dist_Avg_Coh_NQUADECRT_end,Delam Dist_SEM); Delam_SEM_Rel_Diff_Avg_Coh_NQ̈UADECRT_end $:=1.332788777$

...clearly the calculations lead to very small relative errors to the empirical findings providing support for the YDB mechanism...

....end program... 


\section{APPENDIX E: PUBLICATIONS}

Manuscripts in Progress

Krimbalis, P.P. et al. (2009). On a Definition of Tensile Yield as Applied to Glass Reinforced Fibre Metal Laminates.

Krimbalis, P.P. et al. (2009). An Experimentally Investigated Failure Mechanism for the Bearing Strength of Fibre Metal Laminates.

Article Published in Refereed Journals

1. Krimbalis, P.P., Poon, C., Behdinan, Z., Fawaz, Z. (2008). On the Pin Bearing Strength of Orthotropic Fibre Metal Laminates. Journal of Composite Materials, 42(8): 1547-1566.

2. Krimbalis, P.P., Poon, C., Fawaz, Z., Behdinan, K. (2008). The Effects of Thermally Induced Residual Stress on the Fatigue Behaviour of Fibre Metal Laminates. International Review of Aerospace Engineering, 1(2): 216-224.

3. Krimbalis, P.P., Poon, C., Behdinan, K., Fawaz, Z. (2008). On an Experimentally Validated Methodology for Predicting the Strength of Fibre Metal Laminates in Pin Bearing. International Review of Aerospace Engineering, 1(1): $38-45$.

4. Krimbalis, P.P., Poon, C., Fawaz, Z., Behdinan, K. (2007). Experimental Characterization of the Bearing Strength of Fibre Metal Laminates. Journal of Composite Materials, 41(12): 3109-3131.

5. Krimbalis, P.P., Poon, C., Fawaz, Z., Behdinan, K. (2007). Prediction of Bearing Strength in Fibre Metal Laminates. Journal of Composite Materials, 41(9): 11371157.

Other Refereed Contributions

1. Krimbalis, P.P., Poon, C., Fawaz, Z., Behdinan, K. (2008). On the Prediction of Induced Residual Stresses in Fibre Metal Laminates. To appear in: Design, Manufacturing and Applications of Composites: Proceedings of the $7^{\text {th }}$ Joint Canada-Japan Workshop on Composites, Fujisawa, Kanagawa, Japan.

2. Krimbalis, P.P., Poon, C., Fawaz, Z., Behdinan, K. (2008). On the Calculation and Simulation of Thermally Induced Residual Stresses in GLARE Fibre Metal 
Laminates. To appear in: Proceedings of the $7^{\text {th }}$ International Conference on Composite Materials and Technology, Sharjah, U.A.E.

3. Krimbalis, P.P., Poon, C., Behdinan, K., Fawaz, Z. (2007). An Experimentally Validated Methodology for Predicting the Bearing Strength of Fibre Metal Laminates. Proceedings of the $16^{\text {th }}$ International Conference on Composite Materials, Kyoto, Japan, Japan Society of Composite Materials: 1226-1233.

4. Krimbalis, P.P., Poon, C., Fawaz, Z., Behdinan, K. (2006). On the Prediction of Bearing Failure in Fibre Metal Laminates. Design, Manufacturing and Applications of Composites: Proceedings of the $6^{\text {th }}$ Joint Canada-Japan Workshop on Composites, Toronto, Ontario, Canada: 202-209.

Non-Refereed Contributions (Conference Presentations/Web Publications)

1. Krimbalis, P.P., Poon, C., Fawaz, Z., Behdinan, K. (2007). On the Prediction of Bearing Strength in GLARE Fibre Metal Laminates: www.simulia.com/academics/research glare.html

2. Krimbalis, P.P. (2007). Hybrid Composites: The Next Generation of Advanced Aerospace Materials. Presented as an Invited Speaker at the Canadian Aeronautics and Space Institute (CASI): Canadian Student Summit on Aerospace, Toronto, Ontario, Canada.

3. Krimbalis, P.P., Poon, C., Behdinan, K., Fawaz, Z. (2007). An Examination of Residual Stress and the Fatigue Behaviour of Fibre Metal Laminates. Proceedings of the 2007 Winter Workshop of Applied Mechanics, Czech Technical University in Prague, Prague, Czech Republic: http://bulletin-am.fsid.cvut.cz/workshopam/viewpaper.php?id=36\&cf=1: 1-10.

4. Krimbalis, P.P., Poon, C., Fawaz, Z., Behdinan, K. (2006). Prediction of Bearing Failure in GLARE Using a Modified Characteristic Dimension Approach. Presented at the Canadian Society of Mechanical Engineers (CSME) Forum, Kananaskis, Alberta, Canada. Available on CD. 